\title{
Biomimetic Polymer Systems via RAFT Polymerization - Routes to High-Performance Materials
}

\author{
Dissertation \\ zur Erlangung des mathematisch-naturwissenschaftlichen \\ Doktorgrades \\ "Doctor rerum naturalium" \\ der Georg-August-Universität Göttingen \\ im Promotionsprogramm Chemie \\ der Georg-August University School of Science
}

(GAUSS)

vorgelegt von

Michael Hendrich

aus Magdeburg

Göttingen, 2016 


\section{Betreuungsausschuss}

Prof. Dr. Philipp Vana, MBA Institut für Physikalische Chemie Georg-August-Universität Göttingen

Prof. Dr. Konrad Samwer

I. Physikalisches Institut

Georg-August-Universität Göttingen

\section{Mitglieder der Prüfungskommission}

\section{Referent}

Prof. Dr. Philipp Vana, MBA Institut für Physikalische Chemie Georg-August-Universität Göttingen

\section{Korreferent}

Prof. Dr. Konrad Samwer I. Physikalisches Institut Georg-August-Universität Göttingen

\section{Weitere Mitglieder der Prüfungskommission}

Prof. Dr. Michael Buback

Prof. Dr. Burkhard Geil

PD Dr. Thomas Zeuch

Dr. Florian Ehlers
Institut für Physikalische Chemie Georg-August-Universität Göttingen Institut für Physikalische Chemie Georg-August-Universität Göttingen Institut für Physikalische Chemie Georg-August-Universität Göttingen Institut für Physikalische Chemie Georg-August-Universität Göttingen

Tag der mündlichen Prüfung: $\quad$ 02.12.2016 


\section{Summary}

Nature provides numerous examples of structural materials with outstanding mechanical properties. An especially intriguing material is spider dragline silk that outmatches man-made high-performance fibers such as Kevlar or high-tensile steel. Therefore, it has been a prolific source of inspiration to research in various scientific fields.

In this thesis, the macromolecular structure of spider dragline silk was adapted by preparation of multiblock copolymers with hydrogen bonding domains, and soft, amorphous segments, and their mechanical performance was evaluated via tensile testing. The multiblock copolymer structure was achieved by the use of polyfunctional RAFT (reversible addition-fragmentation chain transfer) agents with trithiocarbonate groups acting as junction points between individual blocks. For comparison, triblock copolymers were prepared with similar block lengths and composition. It was found that the multiblock copolymers show superior mechanical performance, exibiting higher elasticity, tensile strength and toughness. After initial tensile testing and failure of test specimens, samples of both tri- and multiblock copolymers could be regenerated via thermal annealing. Then, significantly enhanced sample toughness was observed which indicated an increased number of hydrogen bonding interactions.

In order to expand the scope of polyfunctional RAFT agents for tailored material design, the RAFT polymerization of polyfunctional trithiocarbonates was explored in closer detail. During polymerization, RAFT groups being connected to polymer segments are redistributed between macromolecular chains and a characteristic distribution of RAFT groups in the polymer is obtained. This concept was used for the preparation of polystyrene samples by mixing a bi- and a polyfunctional RAFT agent in specific ratios. By characterizing the prepared samples via size-exclusion chromatography (SEC) it could be demonstrated, that the resulting molar mass and hence the number of RAFT groups per macromolecule can be tailored. 
To exploit this concept for advanced material design, star-shaped RAFT agent was mixed with polyfunctional RAFT agent and multiblock copolymers of styrene and $n$-butyl acrylate were prepared that exhibited the star-shaped topological features. The mechanical properties of the prepared materials were investigated via tensile testing. Compared with multiblock copolymers obtained from pure polyfunctional RAFT agents, significantly improved material toughness was observed for the materials that were prepared using the novel mixing approach. Additionally, strain whitening of polymer samples could be prevented and it was demonstrated, that the mixing approach yields materials with superior toughness than conventional blends of star and multiblock copolymers. In conclusion, this work could demonstrate excellent versatility of polyfunctional RAFT agents for the preparation of highperformance materials. 


\section{Contents}

Summary

1 Introduction and motivation 1

2 Theoretical background 3

2.1 Spider dragline silk 3

2.2 Multiblock copolymers via polyfunctional RAFT agents 6

2.3 Tensile testing 13

3 Spider dragline silk mimicking block copolymers

$\begin{array}{ll}3.1 \text { Preface } & 15\end{array}$

3.2 Block copolymer design 17

$\begin{array}{lll}3.2 .1 & \text { Preliminary work } & 19\end{array}$

3.2.2 Synthesis of poly(APA-block-methyl acrylate) block copolymers 26

$\begin{array}{lll}3.3 & \text { Tensile testing } & 37\end{array}$

3.4 Healing of polymer samples 43

$\begin{array}{ll}3.5 \text { Conclusions } & 50\end{array}$

4 Tuning the mechanical properties of biomimetic copolymers via crosslinking $\$ 3$

4.1 Preface 53

4.2 Addition of silica nanoparticles to biomimetic multiblock \begin{tabular}{ll} 
copolymers & 61 \\
\hline
\end{tabular}

4.2 .1 Synthesis 58

4.2.1.1 RAFT immobilized silica nanoparticles 60

4.2.1.2 Synthesis of matrix multiblock copolymer poly(APA-

block-methyl acrylate)

4.2.1.3 Synthesis of polymer-grafted nanoparticles 64

$\begin{array}{lll}4.2 .2 & \text { Tensile testing } & 67\end{array}$

$\begin{array}{ll}4.2 .3 \text { Conclusions } & 82\end{array}$ 
4.3 Biomimetic star block copolymers via RAFT polymerization using the Z-group approach $\quad 83$

4.3.1 Synthesis 85

4.3.1.1 Synthesis of poly-APA star homopolymers 86

4.3.1.2 Synthesis of poly(APA-block-methyl acrylate) star diblock copolymers $\quad 91$

4.3.2 Differential scanning calorimetry 94

4.3.3 Tensile testing 96

4.3.4 Conclusions 98

4.4 Multiblock copolymer materials via mixing of polyfunctional and star RAFT agents

\begin{tabular}{lll}
\hline 4.4 .1 & Mixing of poly- and bifunctional RAFT agents & 103
\end{tabular}

$\begin{array}{lll}4.4 .2 & \text { Mixing of polyfunctional and star RAFT agents } & 108\end{array}$

4.4.3 Synthesis of poly(styrene-block- $n$-butyl acrylate) copolymers 111

4.4.4 Tensile testing 115

$\begin{array}{ll}4.4 .5 \text { Conclusions } & 125\end{array}$

5 Future perspectives 127

6 Concluding remarks 133

7 Experimental section $\quad 135$

7.1 Instrumentation 135

7.1.1 Atomic force microscopy 135

$\begin{array}{lr}7.1 .2 \text { Chromatography } & 135\end{array}$

7.1.2.1 Column chromatography 135

7.1.2.2 Size-exclusion chromatography (SEC) 136

7.1.3 Differential scanning calorimetry 136

7.1.4 Dynamic light scattering 137

7.1.5 Lyophilisation 137

7.1.6 Nuclear magnetic resonance (NMR) spectroscopy 137

7.1.7 Tensile testing 137

7.1.7.1 Sample preparation 139

7.1.7.2 Regeneration of poly(APA-block-methyl acrylate) block copolymer samples 140

7.1.8 Thermogravimetric analysis 140

7.2 Commercially acquired chemicals 140

\begin{tabular}{lll}
\hline 7.2 .1 & Monomers & 140
\end{tabular} 
7.2.2 Initiators

7.2.3 RAFT agent 141

7.3 Synthesized substances 141

7.3.1 Synthesis of monomer $N$-Acryloyl-L-phenylalanine (APA)

7.3.2 Synthesis of star RAFT agent pentaerythritoltetrakis(3-(S-benzyltrithiocarbonyl)propionate) (star-RAFT1)

7.3.3 Synthesis of RAFT agent $S, S$-Bis $\left(\alpha, \alpha^{\prime}\right.$-dimethyl- $\alpha^{\prime \prime}-$ acetic acid)trithiocarbonate (DMATC)

7.3.4 Synthesis of RAFT agent $S, S$-bis $\left(\alpha\right.$-methyl- $\alpha^{\prime}-$ acetic acid)trithiocarbonate (MATC) 143

7.3.5 Synthesis of polyfunctional RAFT agents poly-DMATC and poly-MATC 144

7.3.6 Synthesis of silica nanoparticles 144

7.3.7 Synthesis of RAFT functionalized nanoparticles 145

$\begin{array}{lll}\text { 7.3.8 Polymers } & 145\end{array}$

7.3.8.1 Homopolymerizations with APA 145

7.3.8.2 Copolymerizations of APA homopolymer with methy acrylate 145

7.3.8.3 Homopolymerization with methyl acrylate 146

7.3.8.4 Methylation of APA polymer samples 146

7.3.8.5 Aminolysis of APA starpolymers 146

7.3.8.6 Nanoparticle polymerizations 147

7.3.8.7 Homopolymerizations with styrene 147

7.3.8.8 Copolymerizations of styrene homopolymer with $\begin{array}{ll}\text { n-butyl acrylate } & 147\end{array}$

7.3.8.9 Cleavage by reaction with excess radicals 147 



\section{1}

\section{Introduction and motivation}

In 1957, Otto Schmitt coined the term biomimetics after developing a device that mimicked the electrical action of a nerve. ${ }^{1}$ It originates from the ancient Greek word biomimesis, which is composed of bios (life) and mimesis (imitation). ${ }^{1}$ Even though the term and the corresponding scientific field is relatively young, its fundamental idea of getting inspiration from life (nature) has been influencing people for at least millennia. It is known that the Chinese tried to produce artificial silk about 3000 years ago ${ }^{1,2}$ and numerous other examples should follow, such as Leonardo da Vinci's design of flying machi-nes ${ }^{1}$ or the invention of Lotusan, a paint for self-cleaning surfaces, inspired by the Lotus flower. ${ }^{2}$

Silk in particular has always been contributing to the life of humans throughout history. Initially used as currency or in the production of textiles, it has become very important nowadays for high-technology applications such as wound sutures and tissue regeneration.3,4 These applications are motivated by the biocompatibility and excellent mechanical properties of silk such as high tensile strength and great extensibility. In this respect, spider silk is usually considered the masterpiece of evolution because it outmatches any synthetic fibers. ${ }^{3}$ Given that spiders have evolved over the course of 400 million years, optimizing the composition of the silk and the spinning process, this superiority should not be surprising. 5,6 It rather shows that it is 
promising to adapt aspects of spider silk in order to obtain synthetic high-performance materials.

In a simplified picture, the protein structure of spider silk resembles a multiblock copolymer with hard, crystalline and soft, amorphous segments. ${ }^{7,8}$ These different segments form a nanocomposite-like structure in the silk fiber, with $\beta$-sheet nanocrystals being embedded into a soft matrix. The $\beta$-sheet nanocrystals are stabilized by hydrogen bonding interactions. These structural features are achieved by specific amino acid sequences of defined lengths in the natural system. ${ }^{9}$ In order to mimick such macromolecular structures successfully, strategies are required that allow for careful macromolecular design. Such strategies have become available with the development of reversible-deactivation radical polymerization (RDRP) techniques. Using special controlling agents, common industrial monomers can be polymerized to yield welldefined macromolecular structures. Among all RDRP techniques, reversible addition-fragmentation chain transfer (RAFT) polymerization is often recognized as the most versatile and robust technique. The control of the RAFT process is based on a degenerative chaintransfer that induces an equilibrium between propagating radical species and dormant chains that carry a RAFT end-group. A unique feature of RAFT is the availability of polyfunctional RAFT agents that exhibit multiple RAFT groups per molecule. These controlling agents provide the possibility to prepare multiblock copolymers in two polymerization steps, ${ }^{10}$ which is arguably the most effortless approach compared with conventional methods.

In this work it was intended to exploit this unique feature for the preparation of multiblock copolymers that exhibit key features of spider silk. By implementation of hard domains with hydrogen bonding interactions and soft segments it was hoped to provide the novel materials with outstanding mechanical properties and multifunctionality. In addition, the preparation of materials with distinct topological features via polyfunctional RAFT agents should be explored. 


\section{Theoretical background}

In this chapter, the reader is introduced to key subjects of this thesis. In particular, selected properties of spider dragline silk are discussed that render it an ideal model system for biomimetic research. Subsequently, the synthesis of multiblock copolymers via polyfunctional RAFT agents is explained and differences to conventional RAFT polymerization are highlighted. The chapter is concluded with an introduction to tensile testing.

\subsection{Spider dragline silk}

Spider silk is an astonishing material which has evolved over the course of hundreds of millions of years. ${ }^{6}$ Within that time frame, silks were adapted to provide optimum adjustment to environmental conditions and to assure the survival of spider species.5,11,12 Even though spider silk has been recognized as a fascinating material and observed for millennia, it was not until the 1970s that significant scientific effort has been dedicated to its exploration. ${ }^{13}$ This research includes various fields of studies, such as the mechanical properties of spider silk,5,11,14-16 its molecular structure, $7,9,17,18$ its self-assembly 19,20 and processing conditions of the silk, $6,21,22$ to name but a few. Due to the inherent complexity it is impossible to give a comprehensive review on this topic. However, it will be attempted to shed light on the most important 
aspects that contribute to the excellent mechanical properties of spider silk. Thus, the reader is provided with basic information on what biomimetic material design should focus on in this respect.

When discussing the excellent mechanical properties of this natural material, the term spider silk is generically used throughout literature. However, this is imprecise as spiders may be capable of producing up to seven different types of silks, all of them being tailored for different purposes. ${ }^{23,24}$ It is hence not surprising that the mechanical properties may vary tremendously to fit certain demands. The dragline silk is referred to as the mechanically most robust kind of silk. It constitutes the frame of a web and it is also a spider's lifeline.5,11,14,23 In particular, the spider dragline silk of the female, golden orb weaver spider Nephila is considered as the benchmark material and the majority of research focuses on this kind of silk. ${ }^{16,23}$ It is especially impressive because of its high strength, which exceeds steel, and its exceptional toughness that outmatches the man-made high-performance fiber Kevlar. ${ }^{25}$ In this section, any explanations will refer to this specific silk.

How does spider dragline silk obtain its exceptional mechanical proper-ties? On one hand, research attempted to determine the structure of the fiber and its molecular constituents. On the other hand it had to be elucidated, how spiders process these fibers. ${ }^{19,26}$

Dragline silk is a fiber that exhibits a double filament structure.14,15 As the dragline is a spider's lifeline, this is an additional safety mechanism: if one filament should rupture, the other can still carry the weight of the spider.5,14 The filaments themselves show a core-shell structure that comprises an inner and an outer core, and three different layers constituting the shell. ${ }^{19}$ The first two layers, a lipid and a glyco coat, are loosely attached to the skin and do not increase the mechanical stability but provide, for instance, basic protection against microorganisms and may serve as carrier for pheromones. The skin, however, displays a high degree of orientation and is tightly connected to the outer core. It supports the core mechanically and provides significant protection versus microbial activities or other environmental influences. ${ }^{19}$

The inner and the outer core constitute about $90 \%$ of the fiber and consist of two silk proteins, called spidroins. ${ }^{19}$ As these proteins are produced and stored in the major ampullate gland, they are called major ampullate spidroins (MaSp1 and MaSp2).23 The inner core comprises a nanocomposite-like structure, that consists of $\beta$-sheet nanocrystals being embedded into an amorphous matrix.7-9 These $\beta$-sheet 
nanocrystals are formed by hydrogen bonding. Towards the outer core, the content of $\beta$-sheet structures and the degree of orientation of the crystals increase. ${ }^{8,19}$ This is accompanied by the absence of MaSp2 in the outer core regions. The reason for this lies in the primary structure of MaSp2. It contains about $15 \%$ of proline, whereas MaSp1 is lacking proline repeating units, and it has been shown that poly-proline segments impede the formation of $\beta$-sheet crystals. ${ }^{27}$ However, one may ask how the compositionally distinct core regions are formed.

This leads to the sophisticated spinning process, ${ }^{22}$ which scientists have been trying to adapt for the past decades.6,8 Key aspects will be described here, without going into detail about the anatomy. The spidroins are stored as a concentrated (up to $50 \mathrm{wt} \%$ ) liquid crystalline solution in water. ${ }^{8}$ This solution, called spinning dope, experiences shear flow in the spinning apparatus, which causes orientation of crystallistes. As the shear forces are inreasing towards the walls of the spinning apparatus, the degree of orientation increases as well. Before the silk is drawn out by the spider as a fiber, water is removed and elongational stresses are increased, resulting in further orientation and strengthening. After the final drawdown, residual water evaporates and the actual dragline is formed. During the spinning process, the $\mathrm{pH}$ value $^{28}$ is also varied and additives are added to the spinning dope to trigger molecular self-assembly. ${ }^{6,8}$ The spinning process is not only impressive because it provides the dragline with its excellent performance and complex structure. It is also remarkably efficient, being conducted at ambient temperature and pressure, and using water as solvent. Spinning from a liquid crystalline solution requires little energy, renders its viscosity almost independent from temperature and the application of shear forces reduces its viscosity. ${ }^{6}$

It also has to be mentioned that dragline silk exhibits crucial length scales. ${ }^{4,26,29-32}$ First of all, it is a very thin fiber (several microns) which inherently reduces the number of inhomogeneities and hence decreases risk of catastrophic failure. ${ }^{26}$ Additionally, dragline silk is considered a nano-composite material due to the nanoscopic $\beta$-sheet crystals. ${ }^{4,29}$ These exhibit a critical length, that leads to more homogeneous loading of the crystals and a higher amount of energy being required to break them. In this respect, hydrogen bonding is essential as well, because it provides the nanocrystals with self-healing ability. ${ }^{4}$

Hopefully, this text provides an impression of the diversity of spider dragline silk and how different scientific fields may benefit from its characteristics. For instance, the spinning procedure may give valuable 
ideas for the processing of technical polymer fibers. ${ }^{6}$ Polymer chemists on the other hand will continue to adapt structural elements of the silk, $3,9,33-35$ as has also been done in this thesis. The presented considerations refer to dragline silk of the golden orb weaver, which is the most investigated system. However, a novel dragline silk could be identified in 2010 that exhibited more than ten times the toughness of Kevlar, even surpassing the golden orb weaver. ${ }^{25}$ The authors could only speculate about the origin of the superior performance, but stated, that there is a significant likelihood of discovering even better performing silks in the future. ${ }^{25}$ It can be readily assumed that dragline silk will remain a prolific source of inspiration for forthcoming generations of scientists. ${ }^{8,23,36}$

\subsection{Multiblock copolymers via polyfunctional RAFT agents}

With the advent of living and controlled polymerization techniques, tailored macromolecular design has become a growing research field of polymer chemistry. Sophisticated structures like star polymers are accessible that are used in various applications such as drug delivery ${ }^{37}$ and molecular imaging. ${ }^{38}$ Block copolymers constitute another fascinating polymer architecture that finds industrial application as compatibilizers ${ }^{39-42}$ for polymer blends or adhesives. ${ }^{39}$

As the name suggests, block copolymers comprise at least two different monomers that form distinct chain segments of homogeneous composition. Depending on the number of blocks that are interconnected, di- and triblock or multiblock copolymers can be distinguished. Block copolymers can also be of interest due to their mechanical properties. When block copolymers comprise soft, amorphous segments and hard, crystalline segments, they may be used as so-called thermoplastic elastomers. ${ }^{43-47}$ As opposed to conventional elastomers, thermoplastic elastomers do not exhibit chemical but physical crosslinks, that is, they can be formed reversibly. The physical crosslinks are formed by the hard segments, for instance, via hydrogen bonding interactions. ${ }^{43,44}$ 
initiator decay and initiation

$$
\begin{aligned}
& \mathrm{I}_{2} \longrightarrow 2 \mathrm{I}^{\cdot} \stackrel{\mathrm{M}}{\longrightarrow} \mathrm{P}_{m}^{\cdot} \\
& \text { pre-equilibrium } \\
& \mathrm{P}_{m}^{\cdot}+\mathrm{Y}^{\mathrm{S}}{ }^{\mathrm{S}} \mathrm{R} \rightleftharpoons \mathrm{P}_{m}^{-} \dot{Y}^{\mathrm{S}} \dot{\mathrm{S}}^{\mathrm{R}} \rightleftharpoons \mathrm{P}_{m}{ }^{\mathrm{S}} \Psi_{Z}{ }^{\mathrm{S}}+\mathrm{R}^{*} \\
& \text { re-initiation propagation } \\
& \mathrm{R}^{\cdot} \stackrel{\mathrm{M}}{\longrightarrow} \mathrm{P}_{1}^{\cdot} \quad \mathrm{P}_{n}^{\cdot} \stackrel{\mathrm{M}}{\longrightarrow} \mathrm{P}_{n+1}^{\cdot} \\
& \text { main equilibrium } \\
& \bigcup_{+M}^{P_{n}^{*}}+Y^{S}-P_{m} \rightleftharpoons P_{n}-Y_{Y}^{S}-P_{m} \rightleftharpoons P_{n}-Y^{S}+\underbrace{P_{m}^{*}}_{+M}
\end{aligned}
$$

Scheme 2-1 Generally accepted mechanism of a RAFT polymerization with elementary reaction steps. $\mathrm{Z}$ denotes the stabilizing group, $\mathrm{R}$ the re-initiating leaving group, $\mathrm{I}$ an initiator fragment, $\mathrm{M}$ a monomer molecule and $\mathrm{P}$ a polymer chain.

$$
Y^{S}-R
$$

Figure 2-1 Generic RAFT agent structure. $\mathrm{Z}$ denotes the stabilizing group and $\mathrm{R}$ the reinitiating leaving group.

The mechanical properties (see next section) of such thermoplastic elastomers benefit from a larger number of blocks, due to an increased number of interacting sites per chain. ${ }^{48,49}$ Hence, there is great interest in efficient preparation of multiblock copolymers. Due to the more complex architecture, however, significantly greater experimental effort is usually involved in their preparation. ${ }^{10,50-52}$ An established method is the coupling of $\alpha, \omega$-functionalized prepolymers, so-called telechelic polymers. ${ }^{53,54}$ As this polycondensation is a step-growth reaction, it implies several inherent limitations. It is highly challenging to obtain large degrees of polymerization and, therefore, large block numbers. As the effective concentration of end-groups decreases with increasing block length, the maximum applicable block molar mass is also limited 
to about $5000 \mathrm{~g} \mathrm{~mol}^{-1} .54$ With regard to mechanical properties, however, it is desirable to obtain larger block molar masses. ${ }^{55}$

Living anionic polymerization constitutes another common method for the preparation of multiblock copolymers. ${ }^{49,56-58}$ Alternating polymerization of two monomers may yield multiblock copolymers with block numbers that correspond to the number of polymerization steps. Synthesis of copolymers with a large number of blocks therefore becomes extremely laborious. The use of bifunctional initiators can reduce the experimental efffort and, for instance, undecablock copolymers have been prepared in six successive polymerization steps. ${ }^{57}$ It also has to be considered that the influence of side reactions and impurities multiplies with every additional polymerization step.

Using reversible-deactivation radical polymerization (RDRP) techniques, multiblock copolymers are also accessible by successive monomer addition. Though RDRP techniques may be favoured over living anionic poly-merization due to the greater robustness of radical polymerizations towards impurities, the experimental effort remains essentially unaltered. Reversible addition-fragmentation chain transfer (RAFT) polymerization,59-63 however, offers a unique class of controlling agents that allows for the synthesis of AB-multiblock copolymers in only two polymerization steps: polyfunctional RAFT agents. ${ }^{10}$ The generic structure of a RAFT agent is depicted in Figure 2-1. As is shown, RAFT agents comprise a stabilizing $\mathrm{Z}$ group and a leaving group $\mathrm{R}$.

In a RAFT polymerization, a specific amount of RAFT agent is added to a conventional radical polymerization system and the elementary reactions - initiation, propagation and termination - are superimposed by two equilibria that involve RAFT species (see Scheme 2-1). Depending on the monomer, stablizing and leaving group have to be chosen carefully in order to provide effective control through the RAFT process. That is, addition reaction of the initially formed radical species to the original RAFT agent and fragmentation of the leaving group in the preequilibrium has to be fast compared with the chain growth reaction. Additionally, the leaving group has to form an effectively re-initiating radical species. When the original RAFT agent is completely consumed, rapid exchange of propagating radical species and dormant chains has to occur in the main equilibrium, in order to provide all chains with, ideally, identical probability for chain growth. Then, effective control may occur, that is, narrow molar mass distributions of the corresponding polymer are observed. Moreover, the majority of polymer chains will feature the RAFT group if reaction conditions and RAFT agent are chosen correctly. 


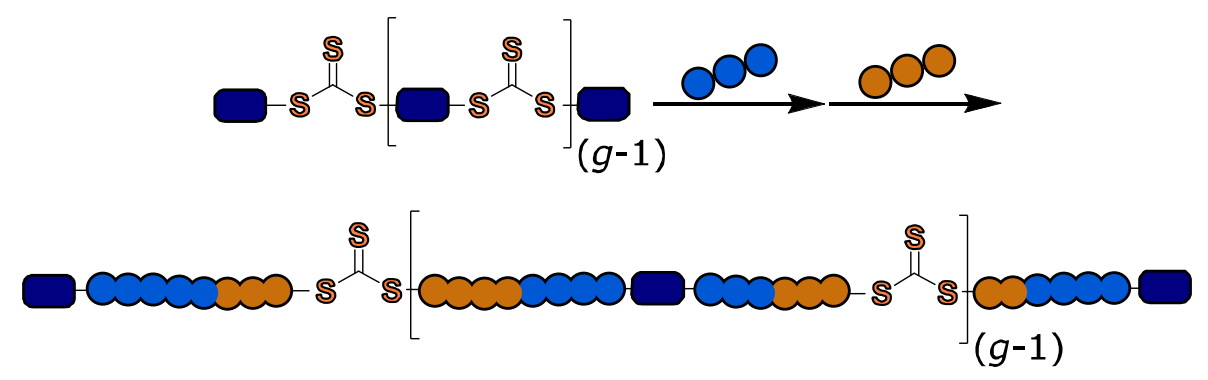

Scheme 2-2 Schematic illustration of a copolymerization process when polyfunctional RAFT agents are applied, showing the formation of AB-multiblock copolymers in two polymerization steps. Here, a polytrithiocarbonate (poly-TTC) RAFT agent ist depicted. Blue and orange circles represent different monomers. The variable $g$ denotes the number of TTC groups per molecule.

High chain-end functionality is also considered an important criterion for effective control, as it allows for post-modification of the polymer material, for instance, by chain extension with a second monomer. ${ }^{64}$ For more detailed insight into the RAFT process, the interested reader is referred to literature given herein.

RAFT agents exhibiting multiple RAFT groups in one molecule are called polyfunctional RAFT agents. These offer access to multiblock copolymers in two polymerization steps, as shown in Scheme 2-2. The groups of You et al.65,66 and Motokucho et al. ${ }^{67}$ were the first to prepare polyfunctional trithiocarbonates (poly-TTCs). Afterwards, Bussels et al. widened the scope of this concept by introducing polyfunctional dithiocarbamtes, which could be applied in emulsion polymerizations. ${ }^{68-}$ ${ }^{70}$ Further studies reported the synthesis of, for example, amphiphilic multiblock copolymers, ${ }^{71,72}$ fluorescent polymers ${ }^{73}$ and nonlinear optical polymers ${ }^{74}$ via poly-TTCs. AB-Multiblock polymers that were obtained using poly-TTCs are also associated with the inherent advantage that TTC groups are excellent binding sites for gold. ${ }^{75}$ Therefore, poly-TTCs allow for the precise arrangement of gold-nanoparticles in gold-polymer nanohybrids yielding superstructures ${ }^{76,77}$ through network formation ${ }^{78}$ or planet-satellite nanostructures. ${ }^{79}$

When polymers that were prepared via polyfunctional RAFT agents are characterized by means of size-exclusion chromatography ${ }^{80}$ (SEC) relatively broad molar mass distributions may be observed, compared with conventional RAFT agents. The observed broadening is the result of a redistribution mechanism being operative when polyfunctional RAFT agents are present (see Scheme 2-3). During the polymerization process, 
blocks and RAFT groups are constantly shuffled until the system is in equilibrium. Therefore, a distribution function $N_{\mathrm{b}}$ of the blocks needs to result after polymerization. $N_{\mathrm{b}}$ corresponds to the number of blocks per macromolecule. Ebeling et al. ${ }^{10}$ determined the expected distribution functions for two limitting cases. Here, key features of that study will be highlighted, as polyfunctional RAFT agents were extensively used for the preparation of multiblock copolymers within the work of this thesis.

To determine the distribution functions, a model system was proposed that included only one radical species. Termination events and other side reactions were excluded as a well-controlled reaction may be presumed. The radical species may add to any RAFT group of a multiblock polymer and form the adduct radical species as shown in Scheme 2-3. Then, fragmentation occurs and a new radical species is formed that attacks another molecule and so on. The refragmentation reaction to the original species is excluded as it does not effect the final block distribution (see Scheme 2-3). In addition, to obtain the block distribution function, two possible mechanisms of how radicals may add to RAFT groups were assumed. In model A, each RAFT group in the system is attacked with equal probability, independently of the macromolecule that it belongs to. A second model B was proposed, where every macromolecule in the system is attacked with equal probability and, consequently, every RAFT group in this molecule then has equal probability of being attacked. Based on these assumptions, two different distribution functions were determined:

$$
\begin{gathered}
N_{\mathrm{b}}{ }^{\mathrm{A}}=\frac{1}{\bar{b}-1}\left(\frac{\bar{b}-2}{\bar{b}}\right)^{b-2}, \\
N_{\mathrm{b}}{ }^{\mathrm{B}}=\frac{4}{\bar{b}^{2}}\left(\frac{\bar{b}-2}{\bar{b}}\right)^{b-2}(b-1) .
\end{gathered}
$$

$N_{\mathrm{b}}$ denotes the number of molecules with $b$ blocks and $\bar{b}$ denotes the average number of blocks. The average block number can be determined, when the average number of RAFT groups $\bar{g}$ per molecule is known, for instance, from SEC characterization. ${ }^{81}$

$$
\bar{b}=\bar{g}+1 .
$$

Ebeling et al. also used the obtained distribution functions to calculate ideal dispersities that would result due to the redistribution 
mechanism, and compared them with expected values for polycondensation reactions. The Schulz-Flory-distribution applies to polycondensation reactions such as the coupling of $\alpha, \omega$-functionalized telechelic polymers. ${ }^{82}$ Figure 2-3 shows the expected dispersities that are obtained for the Schulz-Flory-distribution and the models A and B for polyfunctional RAFT agents. It can be seen that the ideal dispersities approach limiting values of 2 (model A) or 1.5 (model B) for an infinite number of blocks. For large block numbers it is therefore expected that polyfunctional RAFT agents yield polymers with dispersities $\oslash>1.5$, and experimentally determined values should usually lie between 1.5 and 2 .

An additional feature of polyfunctional RAFT agents is the presence of two distinct block species that cannot be interconverted during the redistribution process (see Figure 2-2). When blocks are connected on one end to a RAFT group, they are called end blocks. Blocks that are connected to RAFT groups on both sides are called middle blocks. Depending on the polymerization system, middle blocks may exhibit twice the chain length of end blocks. The presence of the distinct block species has been experimentally shown by Ebeling et al. in a follow-up study, where the authors cleaved the RAFT groups of multiblock polystyrene samples that were obtained via polyfunctional trithiocarbonates. ${ }^{50}$ SEC characterization of the cleavage product yielded a bimodal distribution, proving the existence of the different block species. Additionally, the dispersity of the cleavage product was determined to be 1.18, showing, that blocks of relatively uniform length may be obtained.

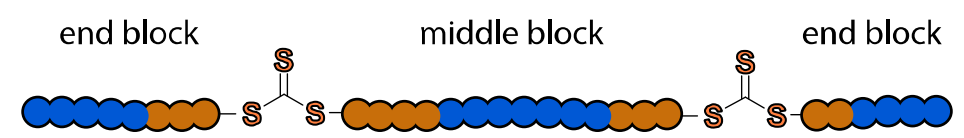

Figure 2-2 Illustration of the two different block species being present in a multiblock copolymer, that is obtained using a polytrithiocarbonate. 


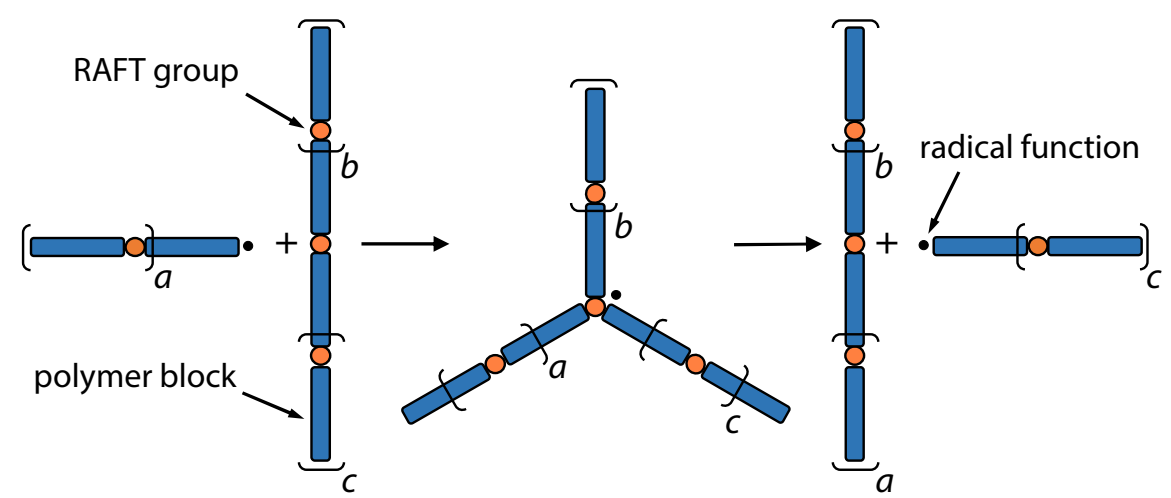

Scheme 2-3 Schematic representation of the redistribution mechanism that is operational in the presence of polyfunctional RAFT agents.

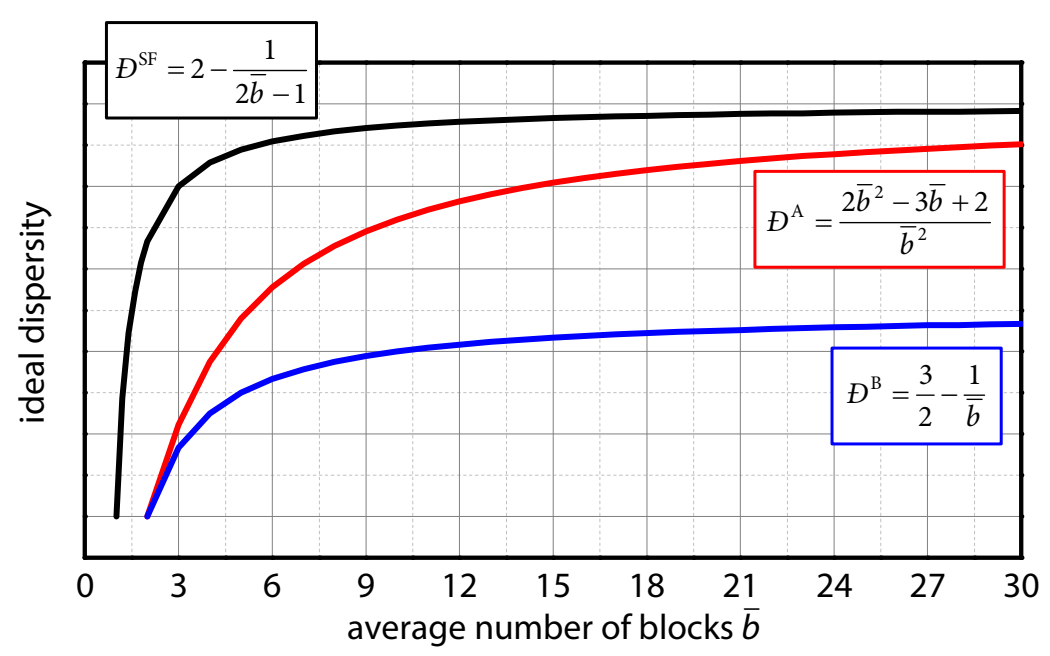

Figure 2-3 Comparison of the ideal dispersities versus the average number of blocks. The respective dispersities correspond to the Schulz-Flory distribution ( $\left.\bigoplus^{\mathrm{SF}}\right)$ or to the specific model for polyfunctional RAFT agents according to Ebeling et al. ( $\bigoplus^{\mathrm{A}}$ and $\left.\bigoplus^{\mathrm{B}}\right) \cdot{ }^{10}$ For further information refer to the text. 


\subsection{Tensile testing}

Tensile testing is a well-established method for the characterization of the mechanical properties of polymers. ${ }^{83}$ It offers access to serveral characteristic quantities that may determine possible applications for a polymeric material. In a typical experiment, rectangular or dogbone*shaped specimens are fixed inside clamps and then elongated at a defined rate. The corresponding elongation $\varepsilon$ is measured as deformation $\left(l-l_{0}\right)$ relative to initial length $l_{0}$ of the sample:

$$
\varepsilon=\frac{\left(l-l_{0}\right)}{l} .
$$

The force $F$ that needs to be applied to the specimen to deform it is recorded simultaneously. Dividing the force $F$ by the initial cross-section $A_{0}$ of the specimen, the stress $\sigma$ is obtained:

$$
\sigma=\frac{F}{A_{0}} .
$$

Even though the cross-section of the specimens is reduced during plastic deformation, the engineering stress, that is usually given, uses the initial cross-section $A_{0}$ throughout the whole experiment. Therefore, the engineering stress is smaller than the true stress once sample deformation occurs.

When the experimentally determined stress is plotted versus strain, characteristic stress-strain curves (see Figure 2-4) are obtained that depend on the molecular structure, composition and molecular weight of the polymer, ${ }^{55,84}$ processing conditions ${ }^{85}$ and testing conditions. ${ }^{83}$ Every polymer exhibits an initial elastic region at very small deformation. Here, any deformation is reversible and, according to Hooke's law, a linear relationship between stress and strain applies:

$$
\sigma=E \cdot \varepsilon
$$

The proportionality constant $E$ is the tensile modulus, which is also termed Young's modulus. Polymers with a high Young's modulus $E$ are termed stiff. After elastic deformation, polymers can exhibit a yield stress $\sigma_{\mathrm{y}}$ which indicates the beginning of irreversible plastic deformation. The maximum load that can be applied to a tensile specimen is termed

\footnotetext{
* In literature, the term is used interchangably with dumbbell.
} 
ultimate tensile strength $\sigma_{\text {UTS }}$ and the corresponding elongation at that point is referred to as strain at break $\varepsilon_{\mathrm{br}}$. Another important quantity is represented by the area under the stress-strain curve. It is called toughness $U_{\mathrm{T}}$ and corresponds to the energy that is absorbed by the tensile specimen:

$$
U_{\mathrm{T}}=\int_{0}^{\varepsilon_{\mathrm{br}}} \sigma \cdot d \varepsilon
$$

When a polymer exhibits a large toughness value it is considered as ductile. Equation (2.7) shows that polymers need to combine large ultimate tensile strength, strain at break and high stiffness, to yield tough materials. This special combination is found in some natural materials such as spider dragline silk (see section 2.1). ${ }^{86,87}$

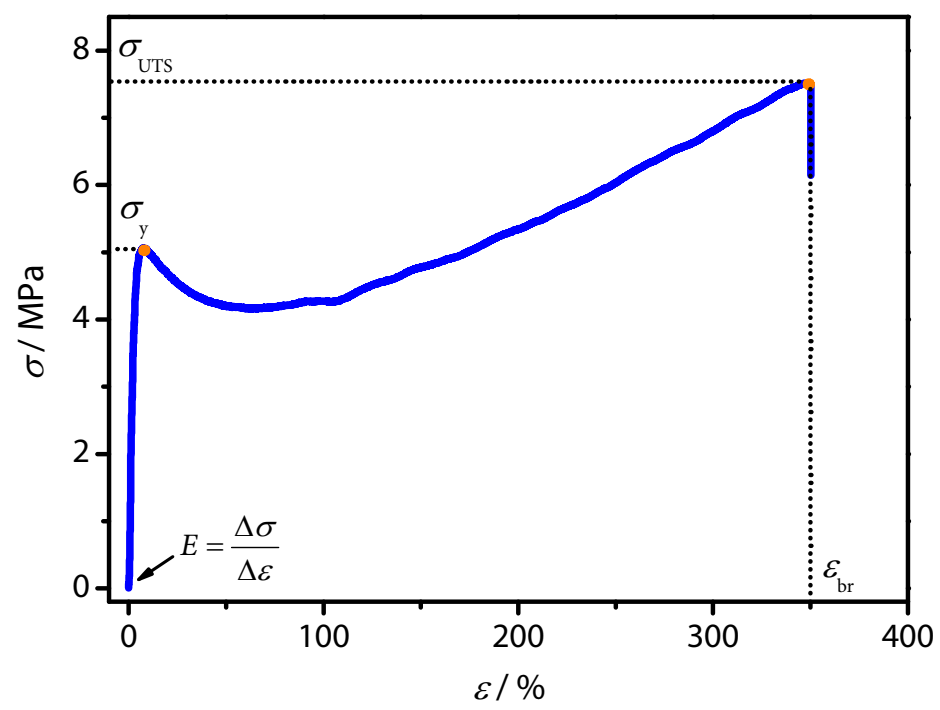

Figure 2-4 An exemplary stress-strain curve with indicated yield stress $\sigma_{\mathrm{y}}$, ultimate tensile strength $\sigma_{\text {UTs }}$ and strain at break $\varepsilon_{\text {br. }}$ 


\section{Spider dragline silk mimicking block copolymers $^{1}$}

\subsection{Preface}

Spider dragline silk is a fascinating natural fiber which outmatches any synthetically obtained fiber concerning its mechanical properties (see section 2.1). ${ }^{23,25,88}$ It is therefore even termed as super-fiber or the holy grail of modern polymer fibers in literature. ${ }^{21,26,89,90}$ One key to its excellent mechanical properties is the molecular structure of spider dragline silk, that is, a multiblock copolymer type structure forming a semi-crystalline polymer (see section 2.1).9,23,33,90 More precisely, spider silk is termed as a nanocomposite material due to the nanoscopic lengthscale of the crystalline polymer segments. 4,32,86,91,92 This length-scale is crucial for the remarkable mechanical properties of spider dragline silk as it results in optimum toughness and strength of the nanocrystals. ${ }^{4}$

Though spider silk offers a multitude of advantageous properties it is to date impossible to produce large quantities for applications. The cannibalistic nature of spiders hampers domestication which has been done for silkworms for millennia to obtain their silk.3,6,23,88 However, even silkworm silks - being inferior to spider dragline silks with respect

\footnotetext{
${ }^{1}$ Adapted with permission from Hendrich, M.; Lewerdomski, L.; Vana, P. J. Polym. Sci. Part A Polym. Chem. 2015, 53, 2809-2819, Copyright 2015, Wiley-VCH Verlag GmbH \& Co. KGaA, Weinheim.
} 
to their mechanical properties ${ }^{3}$ - are produced in vanishingly low quantities compared with commercial polymers such as nylon (about 70000 tons versus several million tons). ${ }^{6}$ Therefore, an artificial production of spider dragline silk is highly desirable. Genetic recombination could be successfully applied to express spider genes in mammalian cells ${ }^{93}$ and different organisms ${ }^{94}$ to obtain artificial spider silk, but a complete expression of spider proteins, called spidroins, has not been achieved yet. Silk fibers spun from artificial spider protein solutions are thus still inferior to their natural analogues although substantial progress has been made in this research field. ${ }^{94}$ It has to be mentioned that genetic recombination is also a rather inapt approach for the production of large quantities of material, at least on an industrial scale. Other approaches aimed towards an emulation of the multiblock structure via step-growth polymerization, connecting disctinct peptide sequences, that have been identified as structural elements in spider dragline silk, ${ }^{90}$ with oligomeric linkers. ${ }^{9,33,95-98}$ The peptide sequences induced discrete secondary structures in the bulk materials that are similar to naturally occurring spider dragline silk. Despite successful structural imitation, the mechanical properties of prepared polymer films were rather poor.9,99

All imitating systems presented so far were very closely related to the actual structure of spider dragline silk. As the mechanical properties of spider dragline silk could not be reproduced adequately it seems to be a limitation remaining too close to the natural model system. Man-made polymer, however, may benefit from a tremendous variety of olefinic monomers if a radical polymerization technique is applied. Using RAFT polymerization as a controlled polymerization technique an additional and unique - advantage is available with a distinct class of controlling agents, namely polyfunctional RAFT agents. These offer a convenient route towards the realization of multiblock copolymer architectures since only two successive polymerization steps are required (see section 2.2).10,50 Thus, the structural implementation of a multiblock copolymer can be achieved with ease. Adequate selection of molecular constituents, however, is a more demanding challenge and requires substantial experimental effort to ascertain suitable combinations of monomers and effective comonomer ratios. This is mainly because material properties cannot be predicted for a novel combination of monomers in a polymer material. Therefore, the present chapter elucidates the design of a spider dragline silk mimicking multiblock copolymer system by starting with selection of monomers, followed by synthetic implementation of 
multiblock copolymers via RAFT polymerization. Subsequently, results of tensile testing of the developed copolymers and differential scanning calorimetry (DSC) measurements are presented and tailoring of material properties is demonstrated by tuning the ratio of the selected comonomers.

\subsection{Block copolymer design}

In order to select suitable monomers for the design of a spider dragline silk-mimicking block copolymer it is worthwhile to consider the natural model system. As described above (see section 2.1 and 3.1), spider silk resembles a multiblock copolymer with hard and amorphous segments. ${ }^{7,9,20}$ Hard, crystalline segments are primarily formed by short alanine strands (four to six repeating units) whereas the soft, amorphous matrix consists of glycine-rich sequences. ${ }^{88,100}$

To form a soft, amorphous matrix, polymer chains have to be capable of moving freely, that is, the polymer exists above its glass transition temperature $\left(T_{\mathrm{g}}\right)$. Investigation of mechanical properties is especially interesting at ambient temperature, a criterion that is met by typical acrylate monomers such as $n$-butyl acrylate (BA, $T_{\mathrm{g}}\left(\text { poly-BA) } \approx-50^{\circ} \mathrm{C}\right)^{101}$ or methyl acrylate (MA, $T_{\mathrm{g}}$ (poly-MA) $\approx 10^{\circ} \mathrm{C}$ )..$^{102}$ Additionally, acrylate monomers have been thoroughly investigated and demonstrated to be polymerizable in a well-controlled fashion via RAFT polymerization, ${ }^{62}$ rendering them ideal candidates for the targeted amorphous polymer segments. Considering the hard, crystalline segments in spider silk, alanine-rich strands are present that are connected via hydrogen bonds as the predominant secondary interaction, providing spider silk with its exceptional toughness.,16,103,104 Hydrogen bonds can be easily incorporated into polymers by selection of corresponding monomers and have already been shown to improve the mechanical properties of polymers and increase their ultimate tensile strength. ${ }^{105}$

Two different monomers were investigated in this work (see Figure 3-1) that both share the advantage of being readily available from commercial substances in one (APA) or two (UPy-MA) synthetic steps. UPy-MA is a well-investigated monomer which has been used in numerous studies to synthesize statistical copolymers101,106-109 or supramolecular polymers ${ }^{110-113}$ and has also found application in a commercially available, self-healing polymer. ${ }^{114}$ This is due to the extraordinarily strong hydrogen bonding of the UPy-group that exhibits four bonding sites forming a self-complementary hydrogen bonding 
motif (see Figure 3-1).115-118 APA on the other hand does not exhibit selfcomplementary hydrogen bonds but also possesses four hydrogen bonding sites and, consequently, appears to be well-suited for the formation of hard polymer segments.

The group of Mori investigated the RAFT polymerization of APA in detail,119,120 and subsequent work utilized APA homopolymers in drug delivery systems, ${ }^{121}$ or APA-containing, amphiphilic block copolymers to obtain pH-responsive vesicles 122 and investigated the chiroptical properties 123,124 of these polymers in solution. Following the given preliminary considerations, it was decided to utilize BA and MA (for the formation of amorphous segments) and UPy-MA and APA (for the formation of hard segments) in initial work to establish a biomimetic copolymer system.

For the realization of multiblock copolymers the RAFT technique was selected, as multiblock copolymers can be prepared very conveniently using polyfunctional RAFT agents (see section 2.2). ${ }^{10,50}$ Here, polyDMATC (see Figure 3-2) was used as polyfunctional RAFT because it has been shown by Liu and Cavicchi ${ }^{81}$ that it may contain a large average number of trithiocarbonate (TTC) groups (up to 20), that is, multiblock copolymers featuring a large average block number may be produced. This is important as higher average block numbers lead to superior mechanical performance. 48,125 It is also easily available from its precursor DMATC (see Figure 3-2) via polycondensation reaction with 1,6-hexandiole.<smiles>C=C(C)C(=O)OCCNC(=O)Nc1nc(=O)cc(C)[nH]1</smiles>

UPy-MA<smiles>C=CC(=O)N[C@@H](Cc1ccccc1)C(=O)O</smiles>

APA<smiles>C=CC(=O)OCCCC</smiles>

BA<smiles>C=CC(=O)OC</smiles>

MA

Figure 3-1 Hydrogen bonding monomers and acrylate monomers that were applied for the design of biomimetic copolymers. Depicted are a methacrylate-type monomer carrying the ureidopyrimidone endgroup (UPy-MA), $N$-acryloyl-L-phenylalanine (APA), $n$-butyl acrylate (BA) and methyl acrylate (MA). 
<smiles>CC(C)(SC(=S)SC(C)(C)C(=O)O)C(=O)O</smiles>

DMATC<smiles>CC(=O)C(C)(C)SC(=S)SC(C)(C)C(=O)OCCCCCCOC(C)(C)C</smiles>

poly-DMATC

Figure 3-2 Applied bi- and polyfunctional RAFT agents DMATC and poly-DMATC for the design of biomimetic copolymers.

\subsubsection{Preliminary work}

In this section, the implementation of the actual comonomer system that was applied for the biomimetic copolymers is elucidated. Therefore, especially unsuccessful approaches are explained which were mainly attempted in the corresponding master thesis. ${ }^{126}$ Hence, synthetic routes are not explained in full detail in this section but the reader is provided with a comprehensive description of the selected proceeding.

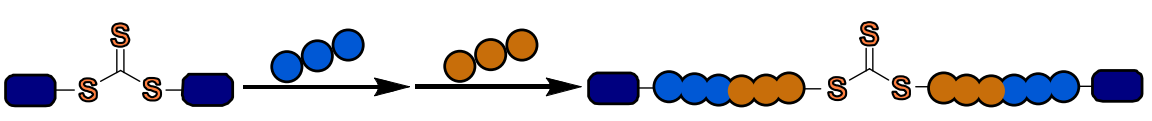

Scheme 3-1 Schematic illustration of a copolymerization process when bifunctional RAFT agents are applied, showing the formation of triblock copolymers in two polymerization steps. Blue and orange circles represent different monomers.

Prior to the preparation of the block copolymers two major points had to be considered regarding the mechanical testing and the synthesis. As explained in the introductory part of this chapter (see section 3.1), tensile testing (see section 2.3) was used to characterize the mechanical properties of polymer samples. Tensile testing requires polymer specimens that exhibit dimensional stability. That means they must not be too brittle (like ceramics or glasses) or too soft (liquid-like) but ductile, to be fixable inside the clamps of a tensile testing machine (see Figure 3-4). With respect to the polymer synthesis the order of the monomers has to be chosen carefully. The macromolecular RAFT agent (macro-RAFT) which is formed in the first polymerization step must have the ability to effectively reinitiate in the second polymerization step, that is, form a good leaving group with respect to the second monomer. ${ }^{127}$ Considering the applied monomers, UPy-MA yields tertiary radical species and APA, BA and MA yield secondary radical species. This means that UPy-MA may only be used in the first polymerization step, since a poly( $n$-butyl acrylate) or poly(methyl acrylate) macro-RAFT 
could not reinitiate poly-merization due to the lower stability of secondary radical species. For APA there is in principle no preference and, consequently, APA may be used in the first or the second polymerization step.

However, the monomer sequence has crucial influence on the mechanical properties of copolymers which comprise more than two blocks. ${ }^{128}$ In Scheme 2-2 the general polymerization scheme is shown to illustrate the position of comonomers along a polymer chain when a polyfunctional RAFT agent is applied. One can see that the monomer which is introduced in the first polymerization step (here depicted as blue circles) will form the end blocks of the corresponding copolymer. The second monomer (here depicted as orange circles) consequently forms linking chains that interconnect the end blocks. A desirable combination of mechanical properties, that is, strength and elasticity, result if the blue blocks display a higher $T_{\mathrm{g}}$ than the orange blocks, and when the copolymer is loaded at a temperature above the $T_{\mathrm{g}}$ of the orange blocks. Then, the blue blocks function as anchor points in the bulk material, providing strength, while the polymer chains of the orange blocks may easily stretch, providing elasticity to the material.

Therefore, block copolymers were synthesized by successive RAFT polymerization of a hydrogen bonding monomer (UPy-MA or APA) and $\mathrm{BA}$ as acrylate monomer. To meet the criterion of dimensional stability for the tensile testing, preliminary experiments were conducted using DMATC as RAFT agent instead of poly-DMATC (see Figure 3-2) to prepare triblock copolymers which represent the smallest repeating unit of a multiblock copolymer (see Scheme 3-1). As already mentioned above (see section 3.1), this proceeding is necessary to determine applicable comonomer ratios because the corresponding mechanical properties can not be predicted.

The prepared triblock copolymer samples are listed in Table 3-1. UPy-MA was modified beforehand by introduction of a triisopropylsilyl (TIPS) protection group to enhance the monomer solubility. All polymer samples were prepared by keeping the number of monomer units constant for the hydrogen bonding monomer (UPy-MA or APA) and varying the BA content. Additionally, the texture of the polymer samples is given as this property indicates if samples are applicable for tensile testing. For UPy-MA-BA copolymers a brittle texture was observed for the first sample, comprising a comonomer ratio of 10 to 100 monomer units. Considering Scheme 3-1 this means that on average short blocks of about five monomer units of UPy-MA form the chain ends (blue 
circles) that are connected by a poly-BA chain (orange circles). The brittle texture of the first samples indicates a strong influence of the hydrogen bonding monomer on the mechanical properties as it leads to the formation of stiff and rigid polymer segments. Increasing the length of the poly-BA chain to 105 monomer units does not alter the texture (see sample UPy-BA-2, Table 3-1), however, an abrupt change in polymer texture is observed when the length of the poly-BA chain is increased to 107 monomer units resulting in a soft and almost liquid-like texture (see sample UPy-BA-3, Table 3-1). This observation was reproducible. Increasing the poly-BA chain length even further did not cause another change in texture. While it is expected that an increase in poly-BA chain length alters the mechanical properties reducing the brittleness of the polymer samples, it is a surprising observation that the samples UPy-BA3 to UPy-BA-5 exhibited a soft and liquid-like structure, indicating a lack of substantial intermolecular forces. One would expect a brittle-to-tough transition with increasing poly-BA chain length, especially with consideration of the delicate changes in poly-BA chain length (see Table 3-1). However, neither of the observed textures for the UPy-MA-BA copolymers is applicable for the preparation of tensile test specimen with dimensional stability.

For the APA-BA copolymer samples a different behaviour was observed (see Table 3-1). Starting at a monomer ratio of 10 to 200 monomer units, that is, the same average end block lengths as in the UPyMA-BA copolymers but an interconnecting poly-BA chain of about twice the length, the polymer texture could always be described as soft but tough. The same texture was observed when the poly-BA chain length was increased even further (samples APA-BA-2 and APA-BA-3, see Table 3-1). Therefore, the polymer materials appeared promising to be suitable for the preparation of test specimen that could be investigated by tensile testing. As described in the experimental section (see section 7.1.7.1), test specimens were prepared via drop-casting method, that is, a concentrated polymer solution is poured into a teflon mold using a pipette and then the solvent is allowed to evaporate slowly. Afterwards, the teflon mold is left in a vacuum oven over night at elevated temperature and under reduced pressure to remove any remaining solvent from the test specimens. The test specimens are obtained as thin (about $1 \mathrm{~mm}$ in thickness) dogbone-shaped samples. Potential drawbacks of this method of sample preparation will be discussed in later chapters concerning the mechanical testing (see section 3.3). 
However, when test specimens of the APA-BA triblock copolymers (APA-BA-1 to APA-BA-3, see Table 3-1) were prepared in the teflon molds, they did not show good dimensional stability and exhibited plastic flow. Thus, it was necessary to modify the comonomer composition of the samples to obtain the required dimensional stability. As the test specimens were too soft, it appeared to be a straightforward proceeding to reduce the overall content of BA. Concerning the monomer UPy-MA it was decided to refrain from further investigations as it was accompanied by, for instance, poor polymer solubility which might have caused problems in the copolymerization process. Additionally, APA-BA copolymers showed the overall more promising performance and handling of monomer emerged to be more facile.

As already mentioned, it was attempted to tune the mechanical performance of the APA-BA triblock copolymers by reduction of the BA content. Additionally, to widen the scope of the investigations, MA was selected as a second acrylate comonomer to prepare APA-MA triblock copolymers which could yield samples with dimensional stability. The prepared copolymer samples are collated in Table 3-2. For the APA-MA copolymers a higher content of MA was applied due to the higher $T_{\mathrm{g}}$ of poly-MA compared with poly-BA (see section 3.1). Hence, at similar chain lengths the influence of APA is more likely to cause brittleness of the copolymer. This reasoning is in accordance with the experimental results (see Table 3-2). While APA-MA-1, comprising a poly-MA chain of about 150 monomer units, displays a brittle texture, APA-BA-4, comprising a poly-BA chain of about 50 monomer units, still shows a rather soft but tough texture. Increasing the poly-MA chain length to 200 monomer units (sample APA-MA-2), a tough texture of the copolymer is restored which is also maintained at a poly-MA chain of 300 monomer units (sample APA-MA-3). The second APA-BA copolymer sample (sample BA-2) also showed a soft and tough texture. Thus, the triblock copolymer samples APA-BA-4, APA-BA-5, APA-MA-2 and APA-MA-3 were used to prepare tensile specimens via drop-casting. However, when trying to remove the specimens out of the teflon molds, APA-BA-2 still turned out to be too soft and respective specimens did not show sufficient dimensional stability. APA-BA-1, APA-MA-2 and APA-MA-3 yielded specimens with good dimensional stability and were consequently investigated via tensile testing. Stress-strain curves are depicted in Figure 3-3. 


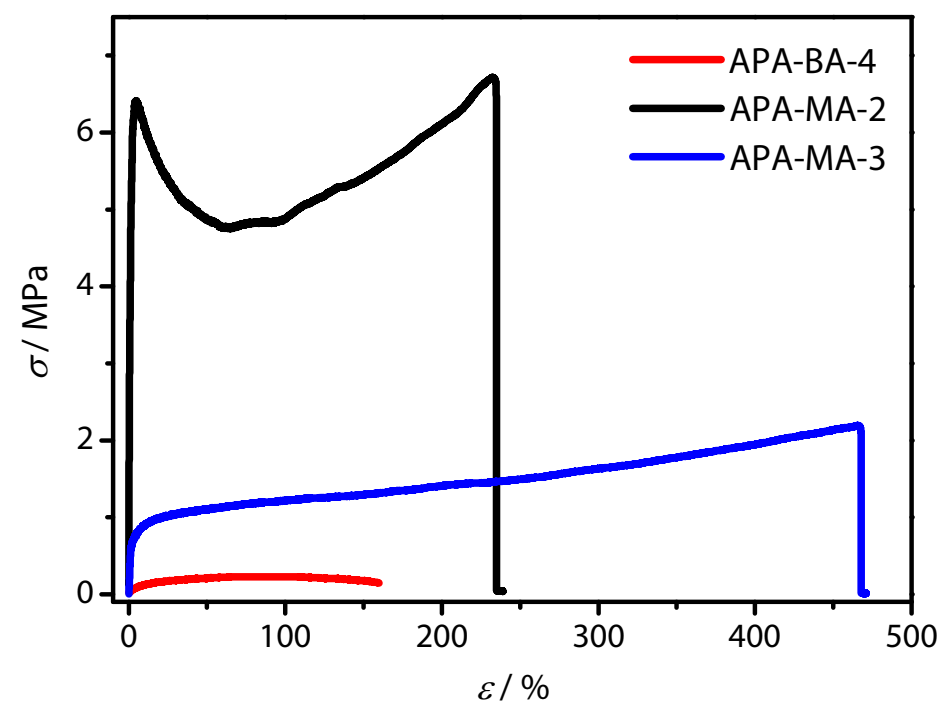

Figure 3-3 Stress-strain curves of copolymer samples APA-BA-4 (red), APA-MA-2 (black) and APA-MA-3 (blue). For corresponding samples see Table 3-2.

The APA-BA triblock copolymer shows a substantially different tensile behavior than the APA-MA triblock copolymers. After a small increase in stress, the sample deforms plastically and the sample diameter is reduced, leading to an apparent decrease in stress. This is observed because engineering stress-strain curves are shown instead of true stress-strain curves (see section 2.3). The sample breaks at about $160 \%$ elongation and a low tensile stress of approximately $0.15 \mathrm{MPa}$. The APA-MA triblock copolymer samples, however, show a pronounced increase of initial stress up to about $6.5 \mathrm{MPa}$ and after a decrease in stress, which is again a result of plastic deformation, the samples display strain-hardening and break at significantly higher tensile stress and elongation than the APA-BA copolymer sample. Simultaneously, the sample toughness, which is represented by the area under the stress-strain curve, of the APA-MA copolymers is significantly superior to the APA-BA copolymers, rendering them the ideal model system for the preparation of block copolymers with enhanced mechanical properties. 
Table 3-1 Overview of prepared triblock copolymers using TIPS-protected UPy-MA (for further explanations see text) and APA as hydrogen bonding monomers, and BA as comonomer. Given are the investigated comonomer ratios, noted as the respective number of monomer units, and the sample textures. (a the sample name includes the description of the hydrogen bonding monomer, $\mathbf{b}$ the ratio corresponds to the respective target degrees of polymerization)

\begin{tabular}{ccc}
\hline \hline sample & $\begin{array}{c}\text { ratio of hydrogen } \\
\text { bonding monomer } \\
\text { to BA }\end{array}$ & texture \\
\hline UPy-BA-1 & $10: 100$ & brittle \\
UPy-BA-2 & $10: 105$ & brittle \\
UPy-BA-3 & $10: 107$ & soft, liquid \\
UPy-BA-4 & $10: 108$ & soft, liquid \\
UPy-BA-5 & $10: 125$ & soft, liquid \\
APA-BA-1 & $10: 200$ & soft, tough \\
APA-BA-2 & $10: 250$ & soft, tough \\
APA-BA-3 & $10: 300$ & soft, tough \\
\hline \hline
\end{tabular}

In summary, the presented preliminary experiments led to the conclusion that the comonomer combination of APA and MA would be ideally suited for the preparation of biomimetic block copolymers in the course of this work. Two major advantages are the good dimensional stability which allows for the preparation of tensile specimens via dropcasting and a promising mechanical performance, including ductility and sample toughness. The next section will elucidate the synthesis of biomimetic block copolymers of APA and MA and also provide a detailed evaluation of their mechanical properties using tensile testing and differential scanning calorimetry as characterization methods. 
Table 3-2 Overview of prepared triblock copolymers using APA as hydrogen bonding monomers and BA or MA as comonomers. Given are the investigated comonomer ratios, noted as the respective number of monomer units, and the sample textures. (a the sample name includes the name of the acrylate monomer, $\mathbf{b}$ the ratio corresponds to the respective target degrees of polymerization)

\begin{tabular}{ccc}
\hline \hline sample & $\begin{array}{c}\text { ratio of APA to } \\
\text { acrylate } \\
\text { monomer }\end{array}$ & texture \\
\hline APA-BA-4 & $10: 50$ & soft, tough \\
APA-BA-5 & $10: 100$ & soft, tough \\
APA-MA-1 & $10: 150$ & brittle \\
APA-MA-2 & $10: 200$ & tough \\
APA-MA-3 & $10: 300$ & tough \\
\hline \hline
\end{tabular}




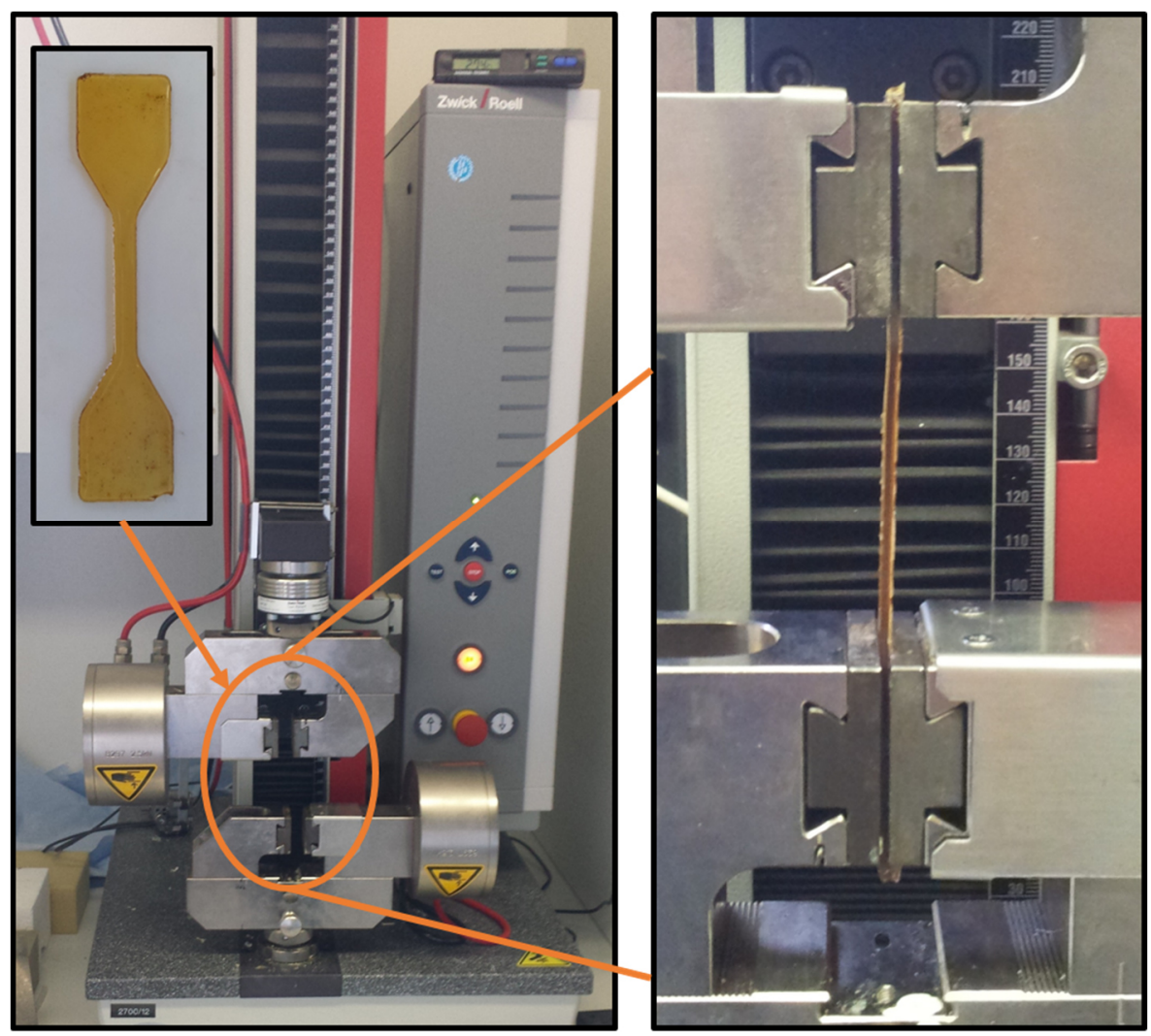

Figure 3-4 Photographs of the utilized tensile testing machine (left) and a test specimen that is fixed inside the clamps (right). Additionally, a typical dogbone-shaped test specimen is shown before testing (top left).

\subsubsection{Synthesis of poly(APA-block-methyl acrylate) block copolymers}

As outlined in the previous section, the combination of APA and MA was evaluated as ideal for the preparation of biomimetic block copolymers. Since the mechanical properties of the copolymers that result from a distinct ratio of APA to MA may not be predicted, triblock copolymers were initially synthesized to evaluate the influence of the comonomer ratio. In the next step, to investigate the influence of the average block number by applying the concept of polyfunctional RAFT agents, multiblock copolymers of APA and MA were prepared. Additionally, to demonstrate the general influence of a hydrogen 
bonding monomer on the mechanical properties, an MA-homopolymer was prepared.

\section{Triblock copolymers}

Triblock copolymer synthesis and the preparation of an MA homopolymer were achieved via RAFT polymerization using DMATC as RAFT agent (see Figure 3-2). Using a bifunctional trithiocarbonate as RAFT agent the preparation of triblock copolymers becomes feasible in two polymerization steps (see Scheme 3-1). For the APA-MA triblock copolymers, APA was polymerized first in the presence of the RAFT agent DMATC. The resulting APA homopolymer was used as macromolecular RAFT agent (APA-Homo) and copolymerized with different amounts of MA to yield corresponding triblock copolymers, exhibiting an APA-MA-APA structure along the polymer chains. For a schematic overview of the polymerization process see Scheme 3-2.

The RAFT polymerization of APA has been investigated by Mori et al.119 in detail to ascertain appropriate reaction conditions for an optimum control of the RAFT process, that is, low dispersity and increase of average molar mass with increasing conversion. Therefore, no additional work was necessary in this regard and the selected polymerization conditions can be found in the experimental section (see section 7.3.8.1). As already outlined in the previous section, a target degree of polymerization of 10 APA monomer units per trithiocarbonate group was chosen. This means for the triblock copolymers that short end blocks of, on average, five APA monomer units are formed that are interlinked by poly-MA chains of varying length (for better illustration see Scheme 3-2). The SEC results of the APA homopolymer (APA-Homo) are listed in Table 3-3. The polymer sample shows an apparent $\bar{M}_{\mathrm{n}, \mathrm{SEC}}=1300 \mathrm{~g} \mathrm{~mol}^{-1}$ and a dispersity $\oslash=1.59$ which is reasonably low for such a highly substituted monomer and with regard to the short targeted chain length. Due to complete consumption of monomer it may hence be assumed that the targeted number of 10 monomer units per RAFT group is obtained in the homopolymer.

Then, the APA homopolymer could be utilized as macromolecular RAFT agent for the copolymerization of MA. The corresponding reaction conditions may be found in the experimental part (see section 7.3.8.2). To realize a different MA content among the copolymers, the target degree of polymerization was varied between 200, 250 and 300 monomer units and polymerizations were conducted up to full monomer 
conversion. The copolymer samples were investigated via SEC (see Table 3-3) and show surprisingly good agreement between the experimental and theoretical $\bar{M}_{\mathrm{n}}$ values. Since SEC was measured versus polystyrene standards this is indicative of a compensation of the different hydrodynamic volumes of APA and MA with respect to styrene. Dispersity values of the copolymers do not differ substantially from the APA homopolymer and therefore indicate a controlled copolymerization. Assuming ideal polymerization kinetics, the theoretical number average molar mass can be calculated via the following equation:

$$
\bar{M}_{\mathrm{n}, \text { Theory }}=\frac{[\text { monomer }]_{0}}{[\mathrm{RAFT}]_{0}} \cdot M_{\text {monomer }} \cdot p+M_{\mathrm{RAFT}}
$$

The equation uses the initial concentrations of monomer and RAFT agent, the molar mass of the monomer $M_{\text {monomer, conversion } p \text { and molar }}$ mass of the RAFT agent $M_{\text {RAFT. }}$.

As a reference sample for tensile testing, an MA homopolymer was prepared using DMATC as RAFT agent (for experimental conditions see section 7.3.8.3). A target degree of polymerization of 200 monomer units per RAFT group was chosen and the resulting polymer (MA-Homo) was characterized via SEC (see Table 3-3). The MA homopolymer shows a good agreement between theoretical and experimental $\bar{M}_{\mathrm{n}}$ and low dispersity, clearly indicating an effective control. Since complete monomer conversion was achieved polymer chains may be assumed to exhibit on average 200 monomer units of MA and small deviations between theoretical and experimental $\bar{M}_{\mathrm{n}}$ are presumably a result of end group effects in SEC. 


\section{I) Homopolymerization of APA}
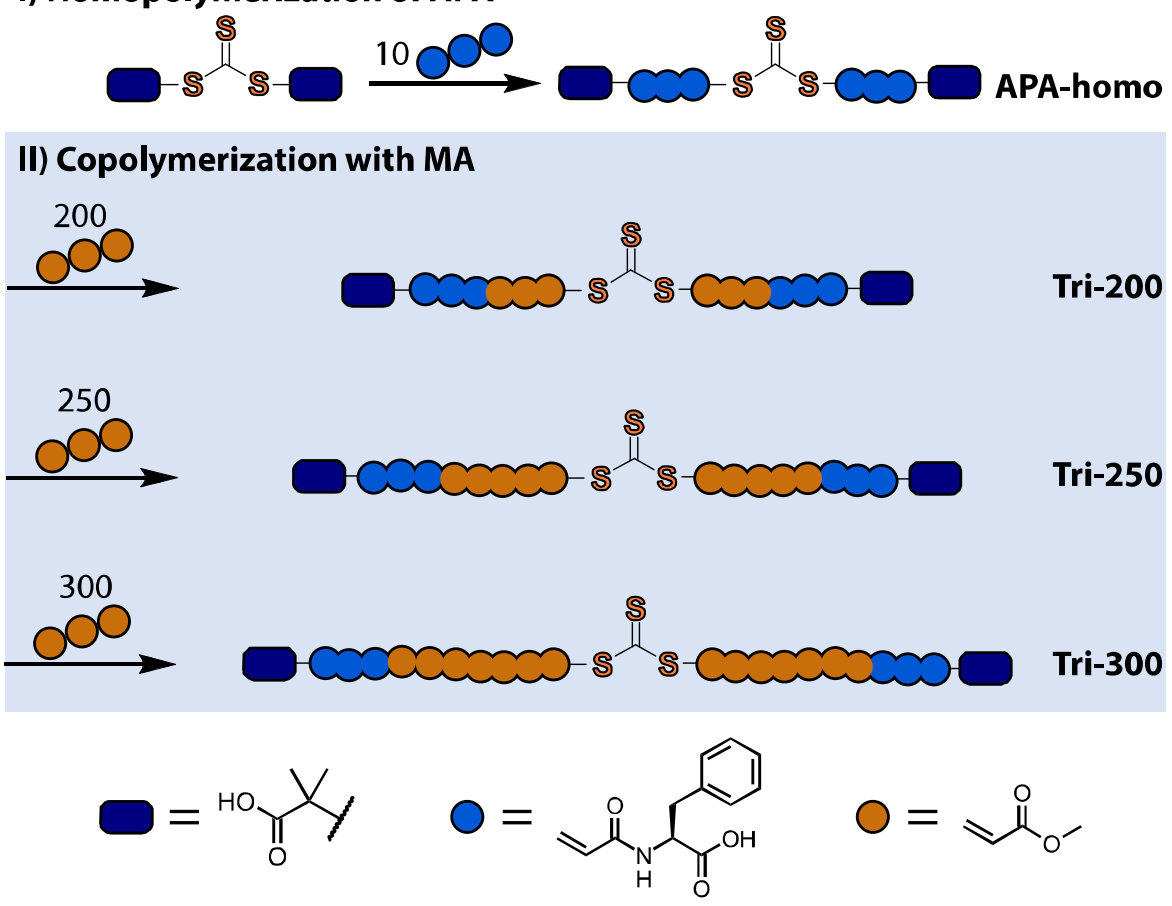

Scheme 3-2 Schematic overview of the synthesis of APA-MA triblock copolymers, showing the preparation of an APA homopolymer (APA-Homo) as macromolecular RAFT agent and subsequent copolymerization with different amounts of MA, yielding three distinct copolymers (Tri-200, Tri-250 and Tri-300). Note that, as depicted here, the APA end blocks ideally exhibit the same length and consist of five APA monomer units, respectively. The same scheme is in principle also valid for the formation of corresponding APA-MA multiblock copolymers. 
Table 3-3 Overview of all copolymer samples that are discussed in this section. Given are the APA homopolymers (APA-Homo and Multi-APA-Homo) which act as macromolecular RAFT agents, the corresponding APA-MA triblock (Tri-200, Tri-250, Tri-300) and multiblock (Multi-200, Multi-300) copolymers and the MA homopolymer (MA-Homo) which serves as a reference sample for tensile testing. SEC was measured versus linear polystyrene standards, except MA-Homo which was measuresd versus poly(methyl acrylate) standards, and results are reported as measured by refractive index detection. $N_{\mathrm{x}}$ indicates the number of monomer units per RAFT group assuming complete monomer consumption.

\begin{tabular}{cccccc}
\hline \hline sample & $\begin{array}{c}\bar{M}_{\mathrm{n}, \mathrm{SEC}} / \\
10^{4} \mathrm{~g} \mathrm{~mol}^{-}\end{array}$ & $\begin{array}{c}\bar{M}_{\mathrm{n}, \text { Theory }} / \\
10^{4} \mathrm{~g} \mathrm{~mol}^{-1}\end{array}$ & $Ð$ & $N_{\mathrm{APA}}$ & $N_{\mathrm{MA}}$ \\
\hline APA-Homo & 0.13 & 0.26 & 1.6 & 10 & - \\
Tri-200 & 1.56 & 1.97 & 1.6 & 10 & 200 \\
Tri-250 & 2.40 & 2.40 & 1.5 & 10 & 250 \\
Tri-300 & 2.50 & 2.83 & 1.7 & 10 & 300 \\
Multi-APA- & 0.25 & 2.43 & 3.1 & 10 & - \\
Homo & & 17.8 & 2.6 & 10 & 200 \\
Multi-200 & 9.70 & 25.6 & 3.2 & 10 & 300 \\
Multi-300 & 3.70 & 1.75 & 1.2 & - & 200 \\
\hline MA-Homo & 1.50 & & & &
\end{tabular}

\section{Multiblock copolymers}

For the preparation of multiblock copolymers a similar proceeding as for the triblock copolymers was followed. First, an APA homopolymer precursor was prepared using poly-DMATC (see Figure 3-2) as polyfunctional RAFT agent. Poly-DMATC had to be characterized prior to use via SEC to determine the average number of trithiocarbonate groups per RAFT agent. The RAFT agent was introduced by Liu and Cavicchi ${ }^{81}$ who found that characterization via SEC versus polystyrene standards and light scattering, an absolute method, yielded similar results. That is, correct molar masses may be obtained and the average number of TTC groups per RAFT agent may be calculated. SEC characterization of polyDMATC versus polystyrene standards yielded a $\bar{M}_{\mathrm{n}, \mathrm{SEC}}=3300 \mathrm{~g} \mathrm{~mol}^{-1}$ and dispersity $\oslash=2.52$. The number of TTC groups per RAFT molecule is calculated to be 9 on average. 


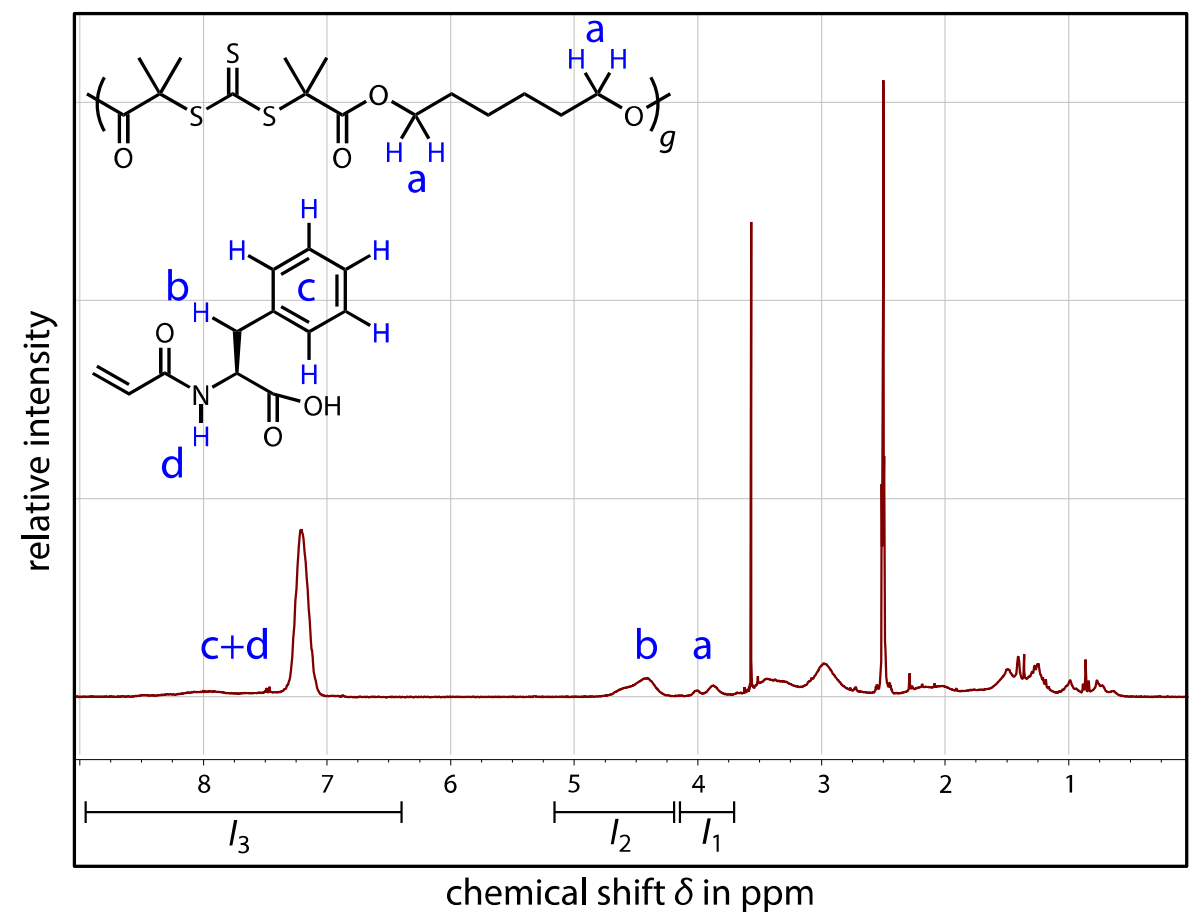

Figure 3-5 ${ }^{1} \mathrm{H}-\mathrm{NMR}$ spectrum of Multi-APA-Homo in DMSO-d ${ }^{6}$ with assignment of proton signals (relative intensities scaled arbitrarily). The integrals that are used in

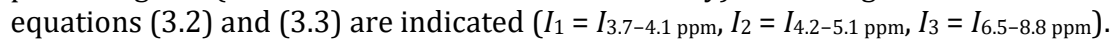

To render the multiblock copolymers comparable with the triblock copolymers it was ensured to use the same target degree of polymerization per RAFT group, that is, 10 monomer units of APA. SEC characterization of the as-prepared APA homopolymer (Multi-APAHomo) revealed a dispersity $Ð=3.1$ and an apparent molar mass of $\bar{M}_{\mathrm{n}, \mathrm{SEC}}=2500 \mathrm{~g} \mathrm{~mol}^{-1}$ (see Table 3-3). The former is not unusual when polyfunctional RAFT agents are applied, as explained earlier in this work (see section 2.2), and is a result of the redistribution mechanism that shuffles RAFT groups among macromolecules during polymerization. ${ }^{10,50}$ The latter finding, however, is remarkable as the apparent molar mass of Multi-APA-Homo is even smaller than that of its precursor RAFT agent. One possible explanation would be an incomplete methylation of the carboxylic acid groups of APA in Multi-APA-Homo, which had to be performed prior to SEC characterization (see experimental section 7.3.8.4). This allows polymer to stick to the SEC column material, leading to longer retention times and hence to a lower 
apparent molar mass. Yet, a successful RAFT polymerization may be assumed since an uncontrolled process would lead to significantly higher molar masses as has been reported by Mori et al.119 for APA.

To give an additional proof for a successful RAFT polymerization, the polymer was characterized via ${ }^{1} \mathrm{H}$-NMR spectroscopy to determine the degree of polymerization. The spectrum of the polymer Multi-APA-Homo and the assignments of the corresponding protons is shown in Figure 3-5. The vicinal methylene protons of the polyfunctional RAFT agent poly-DMATC produce a well-separated signal, corresponding to four protons $\left(I_{1}, \mathbf{a}\right.$ in Figure 3-5). The signal may be compared either to the integral $I_{2}$ of proton $\mathbf{b}$ or the integral $I_{3}$ of the aromatic protons $\mathbf{c}$ and proton $\mathbf{d}$ of the amino functionality (see Figure 3-5) of APA in Multi-APAHomo. When Integral $I_{1}$ is normalized to four protons the degree of polymerization may be calculated using the following equations:

$$
D P_{1}=\frac{I_{2}}{1}
$$

and

$$
D P_{2}=\frac{I_{3}}{6} .
$$

The integers correspond to the respective number of protons present in one repeating unit, that is, 1 for proton $\mathbf{b}$ and 6 for protons $\mathbf{c}$ and $\mathbf{d}$. The degree of polymerization was calculated to be $D P_{1}=10.4$ or $D P_{2}=9.0$ and corresponds well with the targeted value of 10 . It is known from literature that spin-relaxation times of polymers might be substantially prolonged due to hindered rotatability. ${ }^{129,130}$ To ensure reliability of the obtained values, ${ }^{1} \mathrm{H}$-NMR spectra were also recorded with an additional relaxation time of up to $20 \mathrm{~s}$. However, the recorded spectra did not differ significantly proving that this method yields reliable values for the degree of polymerization.

After proving the successful synthesis of Multi-APA-Homo the macromolecular RAFT agent was utilized to produce APA-MA multiblock copolymers. Therefore, two different target degrees of polymerization with respect to MA were selected to produce multiblock copolymer samples that would be comparable with the above discussed triblock copolymers, namely 200 and 300 monomer units of MA per trithiocarbonate group (samples Multi-200 and Multi-300, see Table 3-3). Results of the SEC characterization are collated in Table 3-3. 


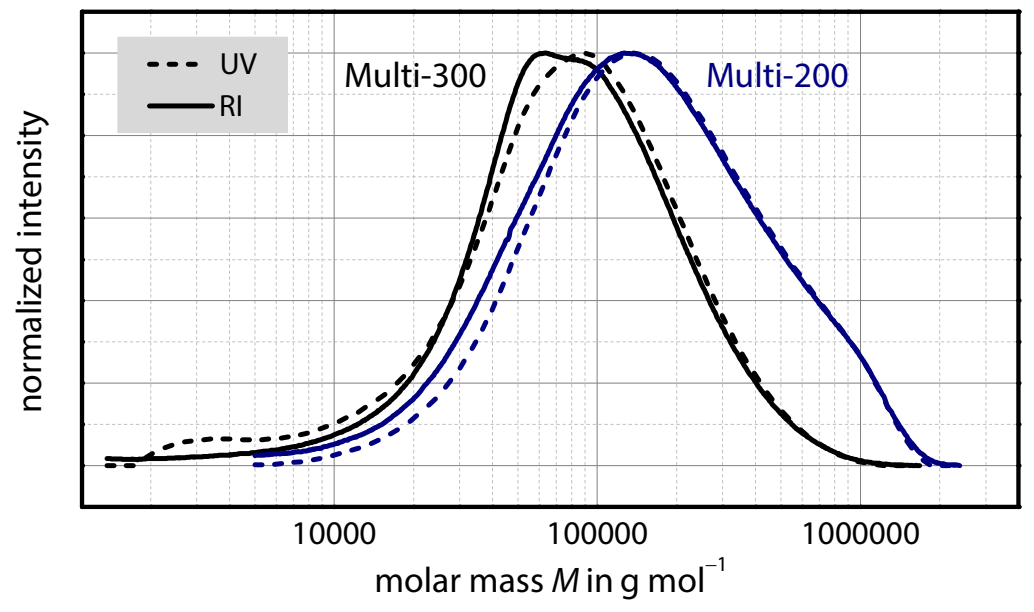

Figure 3-6 SE chromatograms (THF) of the APA-MA multiblock copolymers Multi-200 and Multi-300. UV (wavelength of $\lambda=310 \mathrm{~nm}$ ) and RI detection show good agreement, indicating the presence of RAFT groups along polymer chains.

While the triblock copolymers showed relatively good agreement between theoretical and experimental $\bar{M}_{\mathrm{n}}$ values, the multiblock copolymers show a relatively large deviation (see Table 3-3). This might be again result of an insufficient methylation of the carboxylic acid groups of APA, as observed for Multi-APA-Homo, or this might be a result of the more complex chain architecture resulting in a greater impact on the hydrodynamic volume. Alternatively or additionally, uncertainties in the SEC characterization of the polyfunctional RAFT agent may be effective which could result in a lower true molar mass of the RAFT agent and hence a lower $\bar{M}_{\mathrm{n}}$ of the produced copolymers. The SEC curves of the multiblock copolymers (see Figure 3-6) show a good agreement between RI and UV detection at a wavelength of $\lambda=310 \mathrm{~nm}$ at which trithiocarbonate groups absorb, ${ }^{75}$ and thus confirm the presence of the desired multiblock copolymer architecture. Dispersities of the multiblock copolymers are also larger as for the triblock copolymers, which is in accordance with literature (also see section 2.2).10,50,65,66

In summary the successful synthesis of biomimetic tri- and multiblock copolymers has been presented. Characterization via SEC and NMR affirmed that block copolymers with comparable block lengths have been produced and characterization of their mechanical properties 
hence allows for an investigation of the influence of the block number on the mechanical properties. In addition, an MA homopolymer has been prepared to investigate the general influence of a hydrogen bonding monomer on the mechanical properties.

\section{DSC measurements}

To complement the NMR and SEC characterization of the polymer samples, DSC measurements were conducted to obtain glass transition temperatures of the samples and investigate the influence of APA on the copolymer properties. The DSC curves and corresponding glass transition temperatures are presented in Figure 3-7 and Table 3-4. All polymers display a glass transition temperature $\left(T_{\mathrm{g}}\right)$ close to room temperature, which results from poly-MA segments. All copolymers exhibit an increased $T_{\mathrm{g}}$ compared to pure poly-MA indicating a confiment due to APA blocks which results in a reduced mobility of the poly-MA chain segments. This finding is in accordance with an earlier work that examined triblock copolymers carrying acrylic acid groups as, less effective, hydrogen bonding groups with dynamic mechanical analysis. ${ }^{131}$

Interestingly, the multiblock copolymers Multi-200 and Multi-300 show an additional high temperature transition corresponding to the glass transition of poly-APA segments. This finding suggests the occurence of microphase separation in the multiblock copolymers which has already been observed, for example, in poly(urethane urea) multiblock copolymers. ${ }^{47}$ To explain the absence of an additional glass transition in the triblock copolymers one has to consider the molecular composition along the polymer chains. As described above the target degree of polymerization was 10 monomer units of APA per trithiocarbonate group, resulting in end blocks of, on average, five monomer units in the triblock copolymers. Multiblock copolymers, however, also contain middle blocks that exhibit on average 10 monomer units of APA. This difference between end and middle blocks has already been discussed in section 2.2 and was examined by Ebeling et al.10,50 in detail. Due to the presence of longer poly-APA segments, the multiblock copolymers are consequently more prone to show phase separation. 


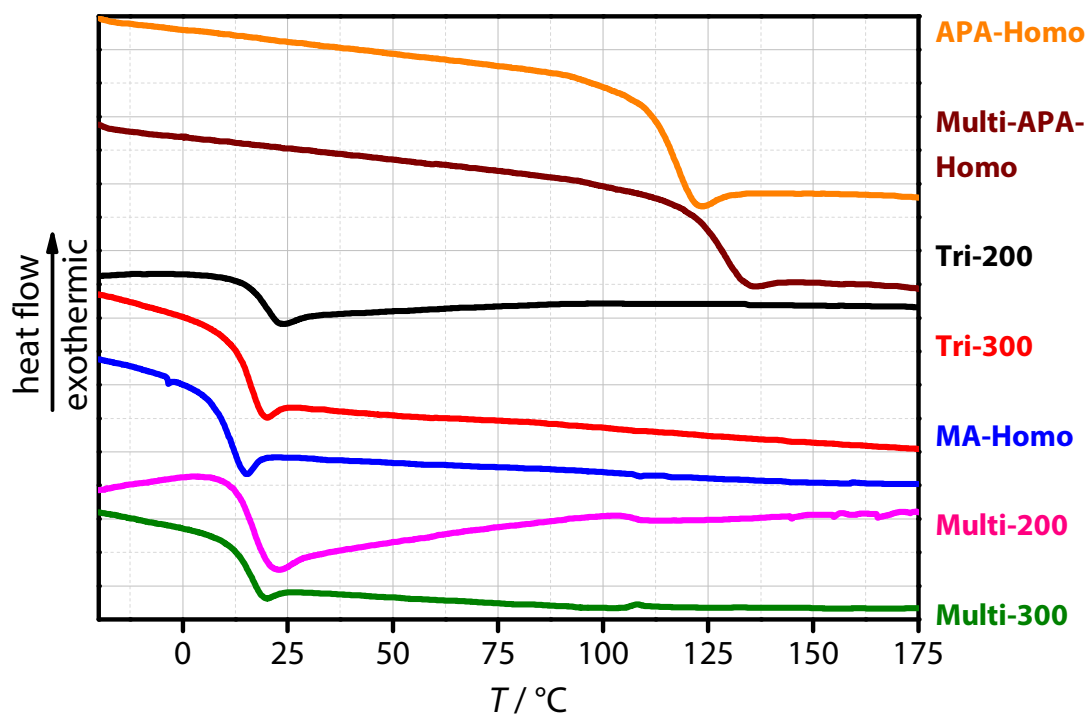

Figure 3-7 DSC curves of polymer samples that are presented in Table 3-3. Curves are shifted vertically for clarity. Glass transition temperatures that were obtained from these curves are collated in Table 3-4.

\section{AFM analysis}

To prove microphase separation of thin block copolymer films, application of atomic force microscopy (AFM) is a commonly selected method.17,132,133 Ideally, distinct morphologies such as lamellar or cylindrical structures can be detected which depend on the comonomer composition of the block copolymers. ${ }^{134}$ The occurence of microphase separation is a result of the immiscibility of the distinct blocks resulting in a thermodynamic driving force to minimize the contact surfaces of the distinct blocks, leading to the aforementioned morphologies. The occurence of microphase separation depends on the interaction parameter $\chi$ (Flory-Huggins parameter), that is, the immiscibility between the comonomers and the degree of polymerization $N$. Additionally, a minimum volume fraction of the minor comonomer component is mandatory for microphase separation to occur. ${ }^{134,135}$

Annealed, thin copolymers films of the biomimetic block copolymers Tri-200, Tri-250, Tri-300 and Multi-200 were investigated via AFM, the respective AFM images can be found in the Appendix A. All samples displayed a smooth and flat coverage of the surface, lacking any 
detectable, regular phase separation. To exclude an influence of the sample preparation process, different annealing temperatures and solvent annealing were used but no visible influence on the polymer surface could be detected. This finding was verified by transmission electron microscopy (TEM) measurements which are not shown here. The absence of a microstructure in the triblock copolymers along with the finding of only one glass transition temperature suggest that the triblock copolymers are not capable of showing microphase separation under the applied conditions. Concerning the above mentioned criteria, it appears reasonable to assume that the volume fraction of the short poly-APA blocks is too low to cause phase separation of the system. ${ }^{135}$ The multiblock copolymers Multi-200 and Multi-300, however, showed an additional glass transition temperature that corresponds to poly-APA segments, hence suggesting microphase separation. Due to the low volume fraction of APA, poly-APA domains should form tiny spheres in the polymer matrix which might be too small to be seen. As Multi-200 did not exhibit any visible phase separation, it was refrained from further AFM investigations into the sample Multi-300 due to the lower volume fraction of APA.

The discussed experimental finding, however, should not be misconceived as a general inability of multiblock copolymers derived from polyfunctional RAFT agents to form microphase separated structures. Within the frame of this work, poly(styrene-block- $n$-butyl acrylate) multiblock copolymers were studied via AFM, which exhibited significantly larger average molar masses $\left(\approx 300000 \mathrm{~g} \mathrm{~mol}^{-1}\right)$ than the biomimetic multiblock copolymers under investigation (see Appendix B). For these systems, phase separation could indeed be detected, which is presumably due to the larger degree of polymerization $N$ (see above). ${ }^{134,135}$

DSC measurements of the polymer samples already demonstrated a significant influence of the hydrogen bonding poly-APA domains on the copolymers by confinement of poly-MA segments. This influence, however, does not lead to a visible phase separation on the microscopic level that could be investigated by AFM. The following section provides detailed investigations via tensile testing and evaluation of the influence of hydrogen bonding sites on the mechanical copolymer properties. 
Table 3-4 Glass transition temperatures of polymer samples (see Table 3-3) that were obtained from the respective DSC curves (see Figure 3-7).

\begin{tabular}{cc}
\hline \hline sample & $T_{\mathrm{g}} /{ }^{\circ} \mathrm{C}$ \\
\hline APA-Homo & 115 \\
Multi-APA-Homo & 127 \\
MA-Homo & 9 \\
Tri-200 & 18 \\
Tri-300 & 15 \\
Multi-200 & 16 and 108 \\
Multi-300 & 15 and 108 \\
\hline \hline
\end{tabular}

\subsection{Tensile testing}

Tensile testing was used to characterize the mechanical properties of the block copolymer samples and the MA homopolymer as a reference sample. Tensile testing is a well-established and frequently used method of materials science to evaluate the mechanical performance of any kinds of materials. Additional information with regard to tensile testing are presented in chapter 2.3. The set-up is depicted in Figure 3-4 along with a typical test specimen exhibiting a dogbone shape. The test specimens were obtained via drop-casting of concentrated polymer solutions in propylene glycol methyl ether acetate (in PGMEA) into dogbone shaped teflon molds. The solvent was allowed to evaporate and the specimens were finally dried in a vacuum oven at $100{ }^{\circ} \mathrm{C}$ and under reduced pressure to remove excess solvent.

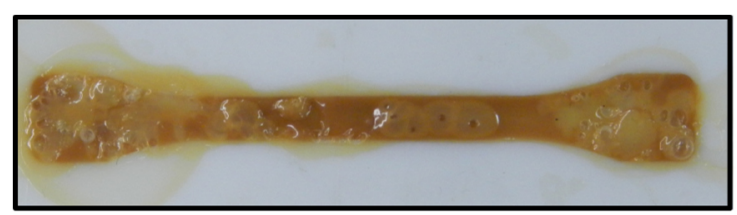

Figure 3-8 Photograph of an inhomogeneous dogbone specimen.

A possible drawback of this method is the formation of inhomogeneous test specimen if the drying process is not conducted patiently. Then, voids may form which cause irregular surfaces of the test specimens rendering them unemployable for tensile testing (see Figure $3-8$ ). This problem is more pronounced for stiffer materials, presumably 
due to more hindered diffusion of solvent molecules. The issue will be commented separately for certain samples.

Tensile testing experiments were conducted at room temperature until failure of the samples applying a constant strain rate of $0.33 \mathrm{~m} \mathrm{~s}^{-1}$. Three samples of each polymer were investigated and characteristic quantities, that is, Young's modulus $E$, strain at break $\varepsilon_{\text {brr }}$, ultimate tensile strength $\sigma_{\text {UTS }}$ and the sample toughness $U_{\mathrm{T}}$ were obtained. Explanations of the characteristic qunatities may be found in section 2.3. First, APA-MA triblock copolymers are compared to MA homopolymer to elucidate the tuning of mechanical properties via implementation of hydrogen bonding sites. Representative stress-strain curves are shown in Figure 3-10. The MA homopolymer exhibits a typical stress-strain curve of a thermoplastic polymer above its glass transition temperature. After a small increase of stress corresponding to the elastic region where Hooke's law applies, the sample practically flows and may be drawn up to the limit of the tensile testing machine. This indicates a lack of internal cohesion.

Changing the macromolecular structure to a triblock architecture containing hydrogen bonding sites in the end blocks alters the observed tensile behaviour substantially. The triblock copolymers exhibit a larger increase of initial stress compared to MA-Homo, which is reflected by larger values of Young's modulus $E$ (see Table 3-5). Subsequently, strain hardening, that is, increase of stress with increasing strain, is observed which ultimately results in a maximum stress, leading to failure of the samples. The strain hardening process is associated with the plastic deformation of polymer chains between physical crosslinks, such as entanglements or hydrogen bonding interactions. ${ }^{136}$ It may also be observed that the hardening process is less pronounced for Tri-250 and Tri-300 compared to Tri-200 resulting in a lower ultimate tensile strength $\sigma_{\text {UTS }}$ (see Table 3-5). This finding can be related to the different chain lengths of the poly-MA chains which the triblock copolymers comprise. It is known, that shorter chains experience a faster orientation along the tensile axis which may result in pronounced strain hardening. ${ }^{137}$ Simultaneously, the strain at break $\varepsilon_{\text {br }}$ increases with increasing poly-MA chain length (see Table 3-5) due to a reduced influence of hydrogen bonding chain segments on the mechanical performance. The sample toughness $U_{\mathrm{T}}$ which corresponds to the area under the stress-strain curve beneftis from an increasing length of the interlinking poly-MA chain as is reflected by the higher toughness values of Tri-250 and Tri-300 compared to Tri-200 (see Table 3-5). 
Table 3-5 Overview of the mechanical properties of tested polymer samples. Characteristic quantities are given as average values and standard deviation that were obtained from three measurements per sample

\begin{tabular}{lclll}
\hline \hline sample & $E / \mathrm{MPa}$ & $\varepsilon_{\text {br }} / \%$ & $\sigma_{\text {UTS }} / \mathrm{MPa}$ & $U_{\mathrm{T}} / \mathrm{MPa}$ \\
\hline Tri-200 & $61 \pm 14$ & $197 \pm 8$ & $3.1 \pm 0.5$ & $5.2 \pm 1.5$ \\
Tri-250 & $54 \pm 56$ & $270 \pm 13$ & $2.9 \pm 0.4$ & $9.5 \pm 5.3$ \\
Tri-300 & $12 \pm 8$ & $474 \pm 138$ & $2.6 \pm 1.7$ & $8.0 \pm 5.5$ \\
MA-Homo & $4 \pm 0.5$ & $>2000$ & $0.3 \pm 0.1$ & $1.7 \pm 3.4$ \\
Multi-200 & $220 \pm 90$ & $302 \pm 14$ & $11 \pm 3$ & $14 \pm 4.5$ \\
Multi-300 & $62 \pm 5$ & $394 \pm 71$ & $8.5 \pm 0.9$ & $13 \pm 0.2$ \\
\hline \hline
\end{tabular}

The presented results suggest an effective formation of physical crosslinks via hydrogen bonding sites which results in increased toughness and ultimate tensile strength of the samples. Additionally, it is shown that the mechanical polymer properties may be tailored by varying the content of hydrogen bonding segments. An increased fraction of hydrogen bonding sites leads to higher ultimate tensile strength $\sigma_{\text {UTS }}$ and lower strain at break $\varepsilon_{\text {br }}$ and vice versa.

Changing the polymer topology to a multiblock architecture the influence of the block number per macromolecule on the mechanical properties can be investigated. Therefore, Tri-200 and Tri-300 are compared to their corresponding multiblock analogues Multi-200 and Multi-300. Typical stress-strain curves are compared in Figure 3-11 and the respective characteristic quantities are shown in Table 3-5. It is evident that the multiblock copolymer samples display higher ultimate tensile strength $\sigma_{\text {UTS }}$ and strain at break $\varepsilon_{\text {br }}$ values than the triblock copolymer samples which combines to an increase of sample toughness $U_{\mathrm{T}}$. Earlier studies already reported an improved performance of multiblock copolymers compared to triblock copolymers with similar block lengths, which is result of a larger number of interacting sites, here poly-APA segments, per macromolecule. ${ }^{48,125}$

Additionally, the higher molar mass $\bar{M}_{\mathrm{n}}$ of the multiblock copolymers compared to the triblock copolymers has to be taken into account. It is known that mechanical properties such as strain at break $\varepsilon_{\mathrm{br}}$ and ultimate tensile strength $\sigma_{\text {UTS }}$ depend on molar mass of polymers due to the formation of entanglements, that is, physical crosslinks. ${ }^{138}$ Entanglements require a minimum chain length to form, ${ }^{139}$ but the maximum density of entanglements which is achievable levels off at a 
certain chain length. 55,140 Therefore, mechanical properties start to benefit at a minimum molar mass from the formation of entanglements but the obtainable improvement is not unlimited. Poly-MA exhibits a socalled entanglement molar mass that corresponds to about 280 monomer units. The presented copolymers contain, at best, on average a comparable number of monomer units with 300 monomer units in Tri300. Hence, the mechanical properties of the presented triblock copolymers might be improved with respect to the multiblock copolymers by increasing the overall chain length while keeping the comonomer ratios constant.

The multiblock copolymers also show a more pronounced strain hardening than the triblock copolymers as can be seen from Figure 3-11. This indicates stronger plastic deformation of polymer chains which agrees with a higher number of physical crosslinks, that is, hydrogen bonding interactions or entanglements as explained above. 55 Furthermore, strain whitening could be observed for the multiblock copolymer samples during tensile testing (see Figure 3-9) which was absent in tests of the triblock copolymer samples. The strain whitening* phenomenon is well-known ${ }^{141-143}$ and has been observed in neat polymers, ${ }^{144}$ rubber modified thermoplastics, ${ }^{145}$ and nanocomposites. ${ }^{141}$ It is the result of optical inhomogeneities 142,145 which cause light scattering, leading to a loss of transparency of a material sample.

Inhomogeneities are often described as crazes which resemble microscopic voids. ${ }^{55,141,143}$ Craze formation occurs during the yielding process of polymer samples and is hence interconnected with plastic deforma-tion. 143,146,147 This is in turn connected to effective formation of entanglements and thus a minimum molar mass is required to observe strain whitening of a polymer sample. 55 The latter explanation agrees well with the observation that stress whitening is absent in the triblock copolymer samples. Strain whitening is reversible by the healing of crazes which occurs when a polymer is allowed to relax. This is in accordance with the observation that test specimens regained optical transparency after sample failure. It has to be noted that strain whitening is undesired with regard to material application due to loss of optical transparency. ${ }^{145}$ A strategy to impede strain whitening is presented in section 4.4 .

${ }^{*}$ In literature the term is used interchangably with stress whitening. Both terms describe the phenomenon of a polymer sample turning turbid during tensile deformation. 

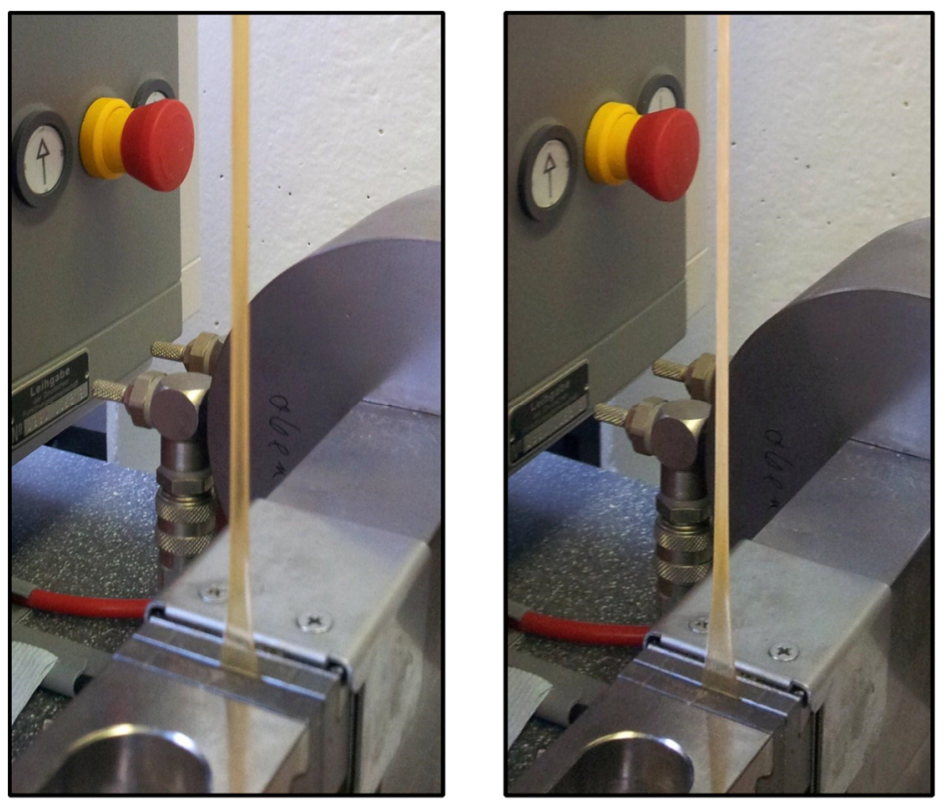

Figure 3-9 Illustration of the stress whitening phenomenon of the multiblock copolymer Multi-300 sample. The left picture shows the starting opaqueness of the polymer sample under tensile deformation. The right picture shows almost complete whitening of the sample. 


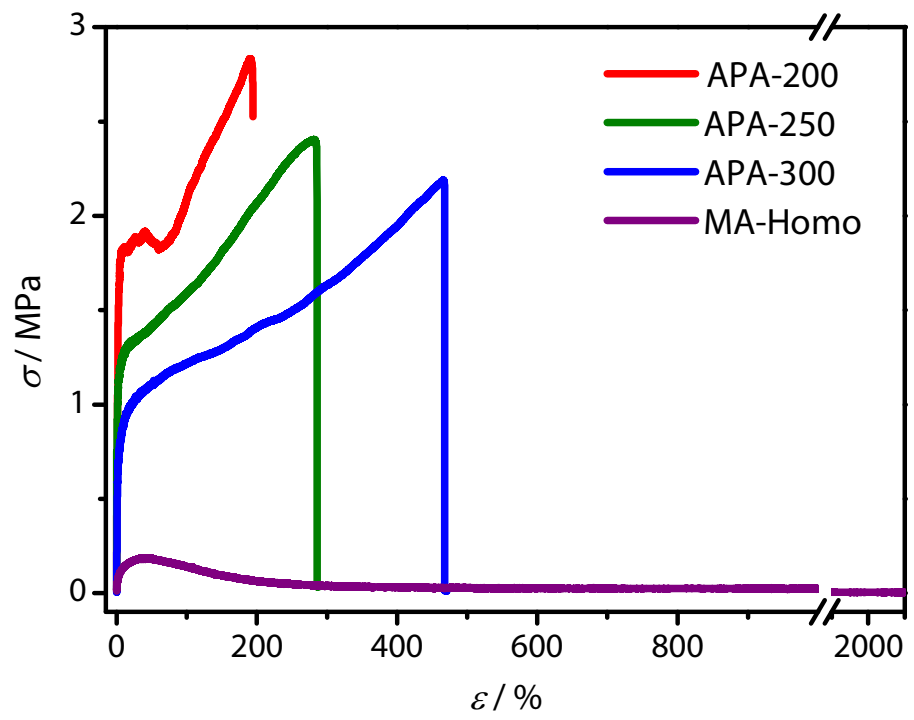

Figure 3-10 Representative stress-strain curves of triblock copolymers Tri-200 (red), Tri-250 (green), Tri-300 (blue) and reference sample MA-Homo. Obtained characteristic quantities from these curves are collated in Table 3-5.

So far, the influence of polymer segments exhibiting hydrogen bonding sites on the mechanical properties of block copolymers has been elucidated. Comparison to a thermoplastic polymer without hydrogen bonding sites (see Figure 3-10) proved the effective formation of physical crosslinks that result in material toughening. Increasing the average block number per macromolecule (Figure 3-11) results in further improvement of mechanical properties due to a larger number of effective physical crosslinks per macromolecular chain. The presence of such a physical network that is formed by hydrogen bonds is, however, not only interesting concerning the mechanical properties but it is also promising to provide the biomimetic block copolymers with shaperegeneration ${ }^{148,149}$ or healing $132,148,150-153$ properties. The following section focuses on the investigation of the biomimetic block copolymers with regard to the aforementioned properties. 


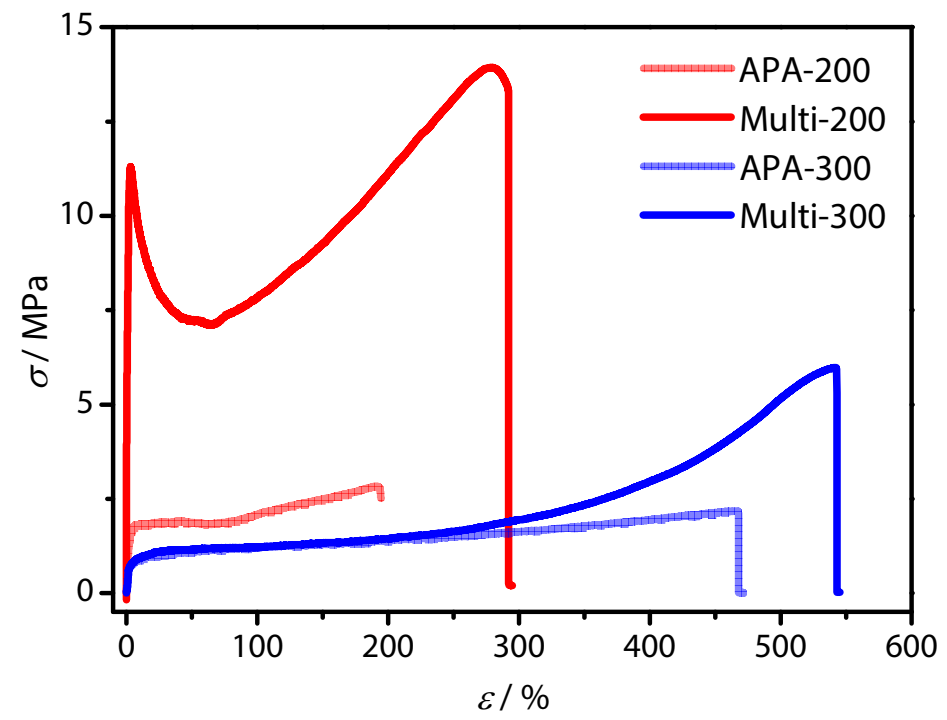

Figure 3-11 Comparison between representative stress-strain curves of triblock copolymers Tri-200 and Tri-300 (transparent curves) and multiblock copolymers Multi-200 and Multi-300 (non-transparent curves). Obtained characteristic quantities from these curves are collated in Table 3-5.

\subsection{Healing of polymer samples}

To check for a regeneration ability of the biomimetic copolymers, the following treatment was applied to the samples after tensile testing until failure: the sample pieces were gently pushed into the teflon mold* so that the fracture sites of the pieces were brought into contact. Afterwards, the samples were annealed overnight, that is, about $16 \mathrm{~h}$ at $100^{\circ} \mathrm{C}$. It has to be mentioned that the pieces retracted almost to their original dimensions at room temperature when the samples were allowed to relax after tensile testing. The sample retraction and the regeneration are illustrated in Scheme 3-3.

* Which was used before in the drop-casting process of the sample. 


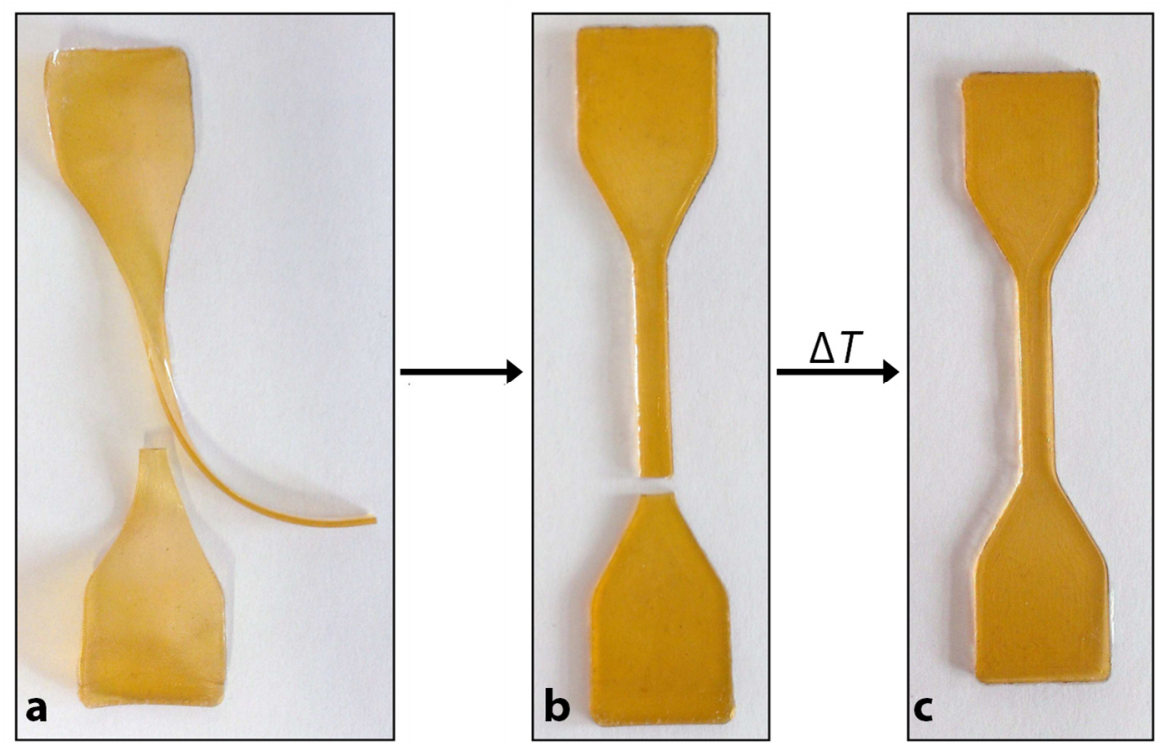

Scheme 3-3 Test specimen of the triblock copolymer Tri-300 (a) shortly after tensile testing, (b) after relaxation at room temperature for several hours and (c) after healing in a vacuum oven at $100{ }^{\circ} \mathrm{C}$ for 12 hours. Note that the regenerated sample (c) does not exhibit any visible flaws. The yellow color of the test specimen is induced by the presence of trithiocarbonate groups.

It is important to note that the test specimens did not exhibit any visible flaws or defects after the regeneration and healing process. Therefore, it appeared obvious to investigate the samples again via tensile testing. Test specimens of Tri-200 and Multi-300 were exemplarily examined to investigate an influence of the healing process on the mechanical properties. It should be pointed out that the here used term "healing" - in materials science also called „welding" 154,155 - must not be confused with "self-healing" which is described as an autonomous process, that is, without external stimulus. ${ }^{*}$ Typical stress-strain curves and the characteristic quantities are shown in Figure 3-12 and Table 3-6 (Tri-200) and Figure 3-13 and Table 3-7 (Multi-300).

Distinct differences may be observed for the stress-strain curves of Tri-200 after the first regeneration. The Elastic modulus $E$ and the ultimate tensile strength $\sigma_{\text {UTS }}$ are increased and, in addition, the

\footnotetext{
* The term "self-healing“ is still used ambiguously in literature. This is supported by the absence of a definition by the International Union of Pure and Applied Chemistry (IUPAC). Therefore, the generic expression „healing“ is used to avoid any incorrectness.
} 
elongation until failure $\varepsilon_{\mathrm{br}}$ of the sample is augmented, leading to a significantly larger sample toughness (see Table 3-6). The copolymer is hence stronger and tougher after the healing process. The observed increase in modulus and tensile strength may be explained by an orientation of the polymer chains in the first tensile test which appears to be memorized even after the annealing process. Such orientation processes are regularly applied to industrial polymers such as nylon fibers ${ }^{156}$ or poly(ethylene terephthalate) ${ }^{157}$ to reinforce the polymers for their application. The strengthening via drawing, however, is usually accompanied by a reduction of the strain at break which is not the case for the bioinspired triblock copolymer Tri-200. An alternative explanation may be suggested when considering the increased sample toughness $U_{\mathrm{T}}$ which represents the energy that is absorbed by the material. This might be interpreted as a larger number of effective intermolecular hydrogen bonds being present after the regeneration process which consequently require a higher amount of energy to be broken. A microscopic interpretation of the observations could be as follows: in the unstressed state, polymer chains try to adapt their thermodynamically most favourable shape which would be a coil. This could lead to a shielding of hydrogen bonding sites which would then form intramolecular interactions.

In the stressed state, however, polymer chains adopt more stretched conformations and segments with hydrogen bonding sites are becoming more exposed to neighboring chains. Hence, the fraction of intermolecular hydrogen bonds that lead to the formation of a physical network might increase and give rise to the enhanced mechanical performance. It has to be noted that sample failure is not a prerequisite to lead to improved sample toughness after regeneration. Deforming a sample to $100 \%$ elongation and then exposing it to a retraction-annealing cycle resulted in a likewise improvement of the respective mechanical properties. 
Table 3-6 Mechanical properties of the triblock copolymer sample Tri-200 in the original state and after subsequent regeneration cycles (see Figure 3-12).

\begin{tabular}{lrlll}
\hline $\begin{array}{l}\text { sample/ } \\
\begin{array}{l}\text { regeneration } \\
\text { cycle }\end{array}\end{array}$ & $E / \mathrm{MPa}$ & $\varepsilon_{\mathrm{br}} / \%$ & $\sigma_{\mathrm{UTS}} / \mathrm{MPa}$ & $U_{\mathrm{T}} / \mathrm{MPa}$ \\
\hline I / & $61 \pm 14$ & $197 \pm 8$ & $3.1 \pm 0.5$ & $5.2 \pm 1.5$ \\
II / 1st & $131 \pm 16$ & $265 \pm 8$ & $9.2 \pm 0.8$ & $18 \pm 3.2$ \\
III / 2nd & $53 \pm 25$ & $385 \pm 59$ & $5.2 \pm 3.5$ & $13 \pm 6.1$ \\
\hline
\end{tabular}

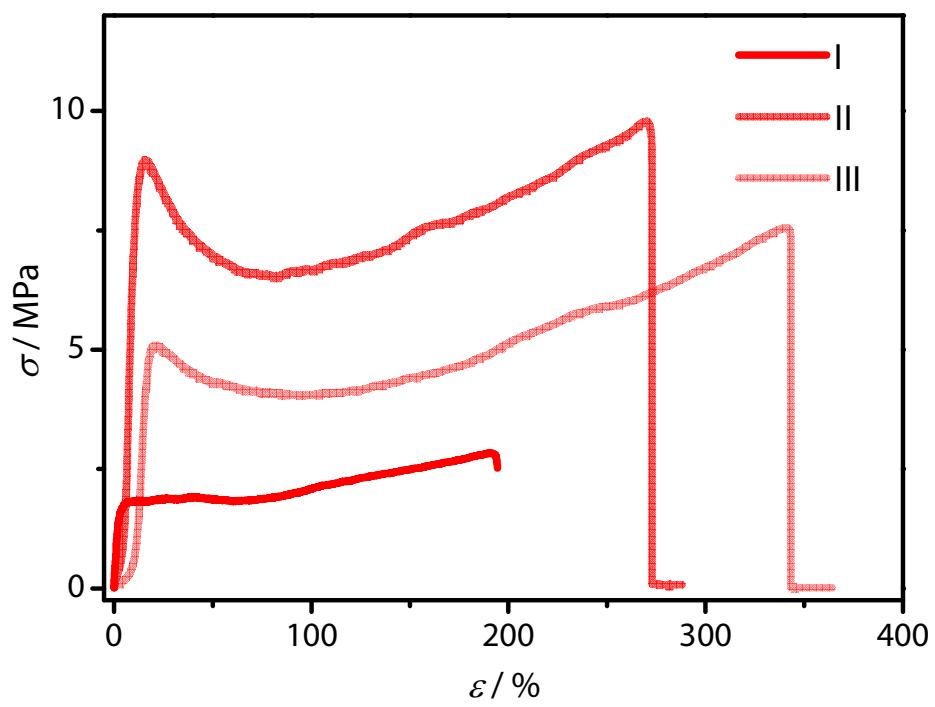

Figure 3-12 Representative stress-strain curves of original (I), once regenerated (II) and twice regenerated (III) triblock copolymer Tri-200 sample. Obtained characteristic quantities from these curves are collated in Table 3-6. Further explanations can be found in the text.

The enhancement of mechanical properties after stretching, breaking and healing of the samples is not an unlimited process since the sample toughness decreases after a second regeneration cycle (see Figure 3-12 and Table 3-6). This is not unexpected as the sample seems to experience fatigue behaviour due to the harsh thermal treatment. Additionally it may be possible that irreversible rupture of polymer chains occurs after sample failure. Though the most pronounced improvement of mechanical properties is observed after the first regeneration cycle, the 
sample properties after the second regenera-tion are still superior to the original sample. This is a remarkable finding as the material may be used multiple times, becoming stronger and tougher after initial failure.

For comparison, the sample Multi-300 was chosen as a multiblock copolymer. Investigation of Multi-200 would have been in principle possible too, however, the preparation of homogenoues specimens was unequally more time-consuming since the samples were very prone towards formation of voids (compare Figure 3-8). This is presumably due to the higher stiffness of the Multi-200 copolymer samples which hinders diffusion of solvent molecules out of the bulk material during the drop-casting process.

Treating Multi-300 copolymer samples with annealing after tensile testing until failure and subsequent retraction led to the same enhancement of mechanical properties with respect to the sample toughness (see Figure 3-13 and Table 3-7). In contrast to the triblock copolymer Tri- 200 a reduction of strain at break $\varepsilon_{\text {br }}$ may be observed and this value cannot be improved compared to the original sample. It is reasonable to assume that the hydrogen bonding sites experience similiar changes like in the triblock copolymers, that is, the number of intermolecular hydrogen bonds increases. This would again explain the significantly larger sample toughness that results after regeneration. Additionally, strain whitening was more pronounced in regenerated tensile specimens of Multi-300 than in original samples. That is, when an original sample was loaded in tensile testing, strain whitening was observed at a strain of about $500 \%$, whereas regenerated specimens exhibited whitening already at about $200 \%$ strain. This means that plastic deformation of polymer chains in regenerated samples of the multiblock copolymer Multi-300 occurs at lower strains which confirms the picture of a physical network with higher crosslink density. The results show that regeneration of the biomimetic multiblock copolymers works similiarly as for the triblock copolymer samples, yielding stronger and tougher materials. 
Table 3-7 Mechanical properties of the multiblock copolymer sample Multi-300 in the original state and after subsequent regeneration cycles (see Figure 3-13).

\begin{tabular}{lcccc}
\hline $\begin{array}{l}\text { sample/ } \\
\text { regeneration } \\
\text { cycle }\end{array}$ & $E / \mathrm{MPa}$ & $\varepsilon_{\mathrm{br}} / \%$ & $\sigma_{\text {UTS }} / \mathrm{MPa}$ & $U_{\mathrm{T}} / \mathrm{MPa}$ \\
\hline I / - & $62 \pm 5$ & $528 \pm 12$ & $6.0 \pm 0.5$ & $13 \pm 0.2$ \\
II / 1st & $130 \pm 7$ & $394 \pm 71$ & $8.5 \pm 0.8$ & $22 \pm 7.1$ \\
III / 2nd & $84 \pm 41$ & $424 \pm 116$ & $7.1 \pm 3.5$ & $17 \pm 0.9$ \\
\hline
\end{tabular}

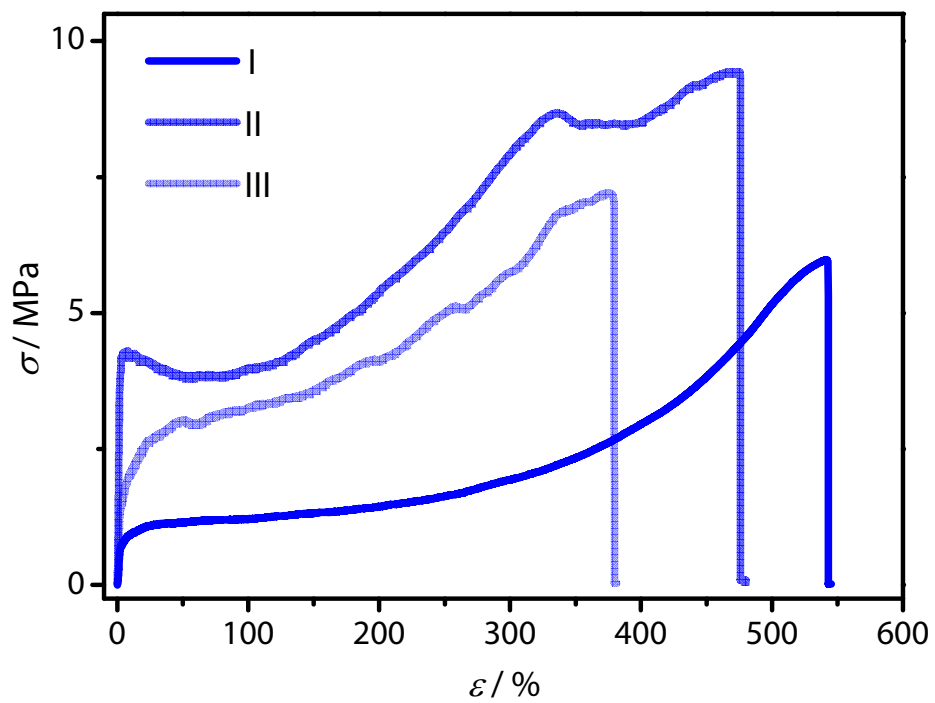

Figure 3-13 Representative stress-strain curves of original (I), once regenerated (II) and twice regenerated (III) triblock copolymer Multi-300 sample. Obtained characteristic quantities from these curves are collated in Table 3-7. Further explanations may be found in the text.

\section{Hysteresis measurements}

Probing the shape stability of a physical network in a polymer and investigation of the ability to absorb energy is possible via so-called hysteresis tensile testing. Multiple loading/unloading cycles are applied to a tensile specimen and the reversibility of plastic deformation may be derived. Here, samples of Multi-300 were investigated via five loading/unloading cycles, straining the sample to $100 \%$ elongation, followed by an initial recovery time of $5 \mathrm{~min}$ at zero load (see Figure 
3-14a). Afterwards, the sample was annealed at $100{ }^{\circ} \mathrm{C}$ for $10 \mathrm{~min}$ before five additional loading/unloading cycles were conducted. Then, the recovery time was prolonged to $60 \mathrm{~min}$ after each cycle at zero load (see Figure 3-14b).

The sample exhibits a large hysteresis in the stress-strain curve, especially, during the first loading/unloading cycle. This indicates an ability of the material to dissipate energy by plastic deformation. Within a recovery time of $5 \mathrm{~min}$ the sample does not regain its initial dimensions and the maximum tensile strength decreases significantly as well as the total energy that is absorbed. Increasing the recovery time to $60 \mathrm{~min}$ leads to a significant enhancement of the shape regeneration ability, resulting in almost identical hysteresis curves after each loading/recovery cycle. This gives additional evidence for network formation via hydrogen bonds acting as physical crosslinks. These are broken during tensile deformation and partially reform hence recovering the original shape to a certain degree. Due to the observed shape regeneration effect, three differenct functions of the hydrogen bonding sites may be identified: (i) on the time scale of the hereperformed experiments, they may form permanent crosslinks resulting in an elastic network that displays shape recovery; (ii) they may open reversibly under mechanical load, leading to an enhanced material toughness compared to, for instance, poly-MA homopolymer and (iii) they facilitate healing of the samples after failure. 

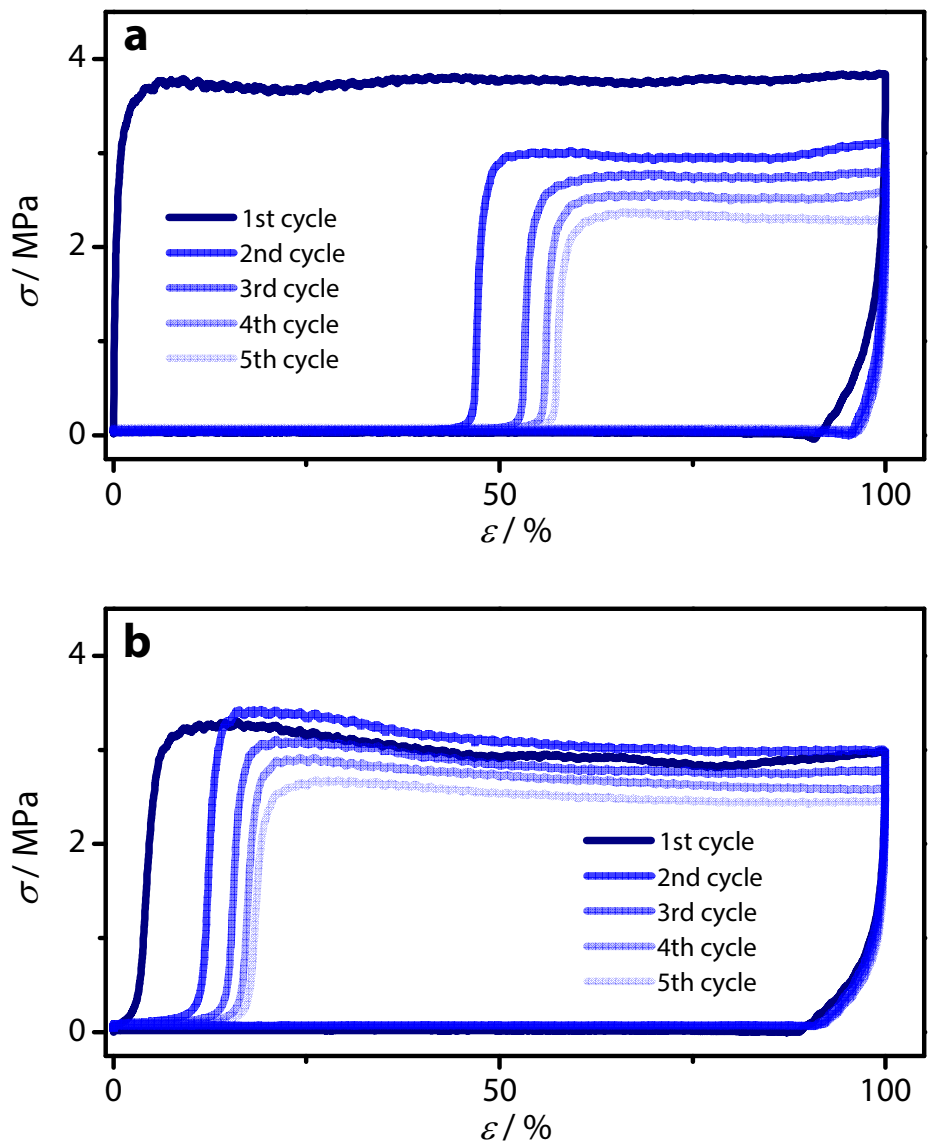

Figure 3-14 Stress-strain hysteresis curves of a multiblock copolymer (Multi-300) specimen with (a) $5 \mathrm{~min}$ and (b) $60 \mathrm{~min}$ relaxation time at zero load at room temperature between consecutive stress-strain cycles.

\subsection{Conclusions}

In summary, following a biomimetic approach of polymer design by using a monomer that carries hydrogen bonding sites, multifunctional materials were obtained that exhibited excellent mechanical properties. It is remarkable that short segments of APA comprising about 5 to 10 monomer units, which corresponds to a content of about $0.1 \mathrm{~mol} \%$ in the copolymers, are sufficient to significantly increase strength and 
toughness of poly(methyl acrylate). This finding is outstanding with respect to monomers that are usually applied to modify the mechanical properties of thermoplastic elastomers such as, for instance, styrene ${ }^{133,158-161}$ which have to be used in significantly larger quantities. Importantly, the hydrogen bonding monomer APA is readily available in a simple one-step synthetic procedure, rendering it an excellent alternative.

Imitating the biological model system spider dragline silk in closer detail by applying polyfunctional RAFT agents to obtain multiblock copolymers, a significant improvement of sample toughness was observed compared with corresponding triblock copolymers. Additionally, the multiblock copolymers exhibited strain whitening during tensile testing which indicates a different deformation mechanism on the microscopic level. Both, triblock and multiblock copolymers, could be healed after failure via thermal annealing and the samples then showed a significant enhancement of toughness. The samples did not display any visible flaws and did not rupture at the same position as in the previous tensile testing cycle, proving complete material regeneration. After regeneration, triblock copolymer samples could be elongated to a greater extent whereas the multiblock copolymer samples showed a slightly decreasing strain at break value.

The reported results suggest that biomimetic material design via RAFT polymerization using polyfunctional RAFT agents are a valuable means towards functional, high performance materials. However, the here presented materials may not be compared to spider dragline silk with respect to their mechanical performance. As has been described in section 2.1, the performance of spider silk is not based only on the sophisticated molecular structure but also on the finely tuned processing of the silk which provides it with a certain degree of orientational order. It has also been demonstrated, for instance, for polyurethanes that sample toughness may be dramatically improved by predrawing of the samples, making them more comparable to spider dragline silk.162 The work in hand, however, focuses on the enhancement of mechanical properties via tailored macromolecular design and modification through different processing procedures is excluded. Therefore, strategies for an additional improvement of the presented biomimetic approach and the resulting material performance are discussed in the following chapter. 



\section{4}

\section{Tuning the mechanical properties of biomimetic copolymers via crosslinking}

\subsection{Preface}

Crosslinking is an additional strategy that is used by nature to modify mechanical properties. Crosslinking in elastin, for instance, provides it with optimum mechanical properties to constitute a connecting material between soft and stiff anatomical elements. ${ }^{86}$ On a molecular length scale, crosslinking is used to stabilize the structure of enzymes such as ribonuclease A and therefore their function. ${ }^{163}$ Probably the best known application of crosslinking is the vulcanization process to obtain rubber materials. It was initially utilized to modify natural rubber latex which itself exhibits only a little fraction of crosslinks. This results in undesirable material properties with respect to the applicable temperature range as the latex turns rigid at low temperatures and sticky at high temperatures. ${ }^{164}$ In addition, natural rubber latex perishes easily. Concerning the natural function of rubber latex, this is readily understandable as the latex is supposed to close and protect wounds of plants being sticky to catch herbivores that could potentially harm the plants.165 Processing via vulcanization of the latex yields rubber materials that retain their stability at elevated temperatures, flexibility at low temperatures and lose their stickiness. ${ }^{164}$ These rubbers turn into tough materials of excellent thermal stability. They can be stretched to 

crosslinking

strains of over several hundred percents without failure and show additional strengthening at large deformations due to strain induced crystallization. ${ }^{166}$ Vulcanization has proved to be an indispensable method for demands of modern society as is reflected by the annual production of billions of tires using natural rubbers, excluding any other application. 167

While the method is powerful and demonstrates beneficial effects of crosslinks on mechanical properties, it inherently yields materials with a fixed shape. However, recyclability and processability might be desirable while keeping the excellent mechanical properties. A different polymer class that combines both characteristics are thermoplastic elastomers. ${ }^{43,44,168-170}$ Like the presented biomimetic copolymers (see chapter 3), these can consist of block copolymers exhibiting soft segments of low glass transition temperature and hard segments of high glass transition or melting temperature. The soft segments assure flexibility over a large temperature range and the hard segments fix the desired shape. When heated above the glass transition or melting temperature of the hard segments, the polymer is readily processable and the material may be turned into any other shape. The formation of physical crosslinks in such materials is illustrated in Figure 4-1a. To improve this approach even further and provide additional crosslinks, star-shaped block copolymers may be used as these inherently contain a chemical crosslink through the star core that interconnects the arms (see Figure 4-1b). This leads to a higher interconnectedness of, for instance, the hard segments because the star arms are preorganized through the core and thus molecular bridging occurs more effectively. ${ }^{171}$ Furthermore, star block copolymers can exhibit higher uniformity of hard and soft segments, which leads to a more homogeneous stress distribution. ${ }^{172}$ This is important as stress concentration causes catastrophic failure of a sample, 30 that is, the material toughness may be elevated in star block copolymers. Eventually, the overall deformation mechanism may be changed when switching from a, for example, linear triblock copolymer to a corresponding star block copolymer structure, also leading to larger sample toughness. ${ }^{173}$ 

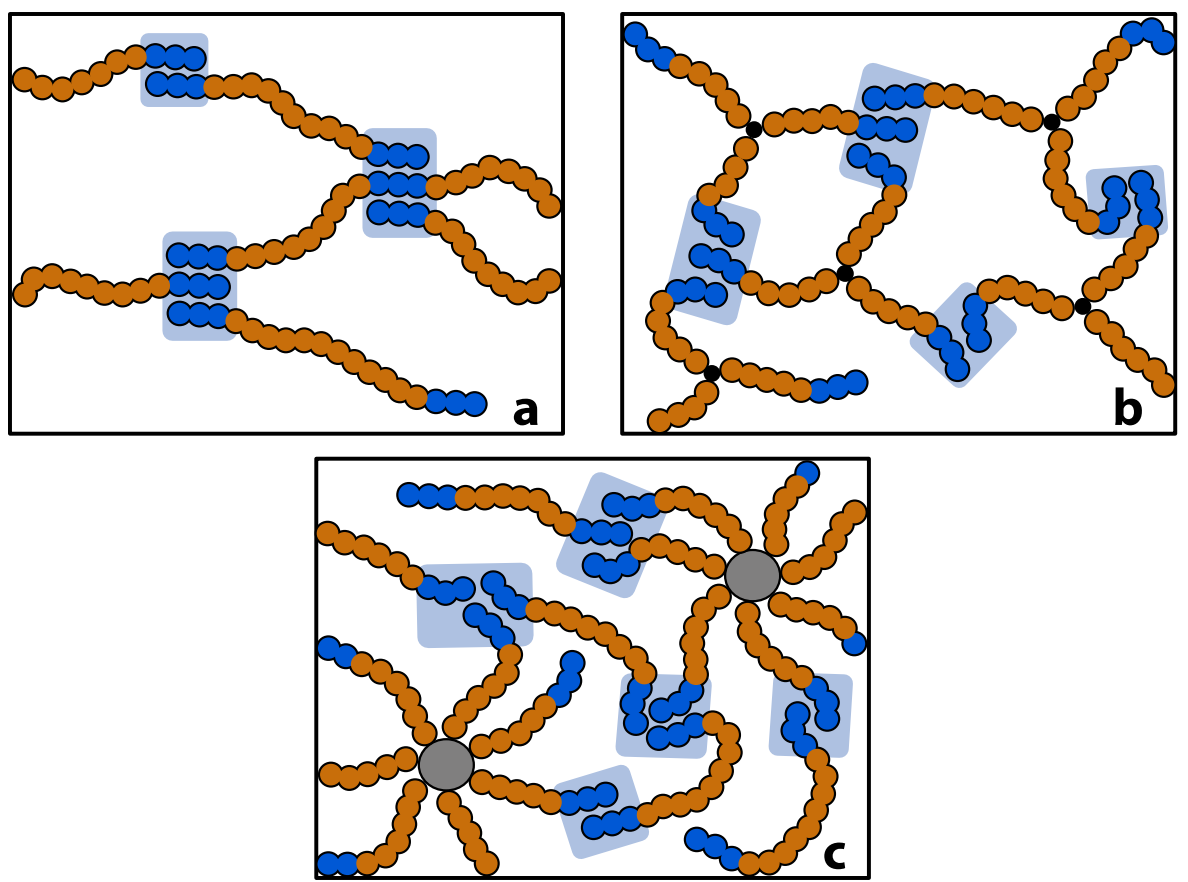

Figure 4-1 Schematic illustration of crosslinking in (a) triblock copolymers via hard domains (blue), (b) 3-arm star block copolymers via chemical crosslinks (black) and hard domains (blue) and (c) polymer-coated, inorganic nanoparticles in a triblock copolymer matrix via nanoparticles (grey) and hard domains (blue). Note, that the drawing does not represent the true size ratios of the components.

A different strategy to improve the mechanical properties of polymers that does not only rely on organic compounds is the addition of polymer-coated inorganic nanoparticles to a polymer matrix. ${ }^{174}$ This approach may also be considered as a possibility to increase crosslinking in a polymer material and hence the mechanical performance, as the nanoparticle cores may resemble the joints of a network (see Figure 4-1c). However, it has to be mentioned that the reasons for the different mechanical properties of such hybrid materials cannot be deduced entirely from such an oversimplified picture.

If one considers a polymer-coated nanoparticle as depicted in Figure 4-1c, there are two counterbalancing driving forces, namely the shortrange enthalpic attraction of the particles and the long-range entropic repulsion of the grafted chains which lose conformational entropy as particles approach one another. ${ }^{175}$ Therefore, polymer-coated nanoparticles can form a variety of different equilibrium structures in a 
polymer matrix, depending on the grafting density and the ratio of the chain lengths of the grafted and the matrix polymer. ${ }^{175}$ While it is generally accepted for unmodified nanoparticles that they have to be well-dispersed in a polymer matrix to yield optimum enhancement of mechanical properties, ${ }^{176-178}$ this does not hold true for polymer-coated nanoparticles.

Above the glass transition of the matrix polymer, it has been found that percolation of the particles, that is, network formation of the particles has to occur. ${ }^{175,179-181}$ This can be understood as particle aggregation which is mediated through the grafted polymer chains. Then, nanoparticles may indeed be considered as junction points of the hybrid network, with the grafted polymer chains providing interfacial adhesion through entanglements. In a recent study it was additionally demonstrated that this interfacial adhesion could be increased by the implementation of hydrogen bonding sites into the grafted polymer chains. ${ }^{182}$ Below the glass transition temperature of the matrix polymer, however, the most beneficial effect on the glassy state modulus of the polymer is achieved when the polymer-coated nanoparticles are welldispersed in the matrix. ${ }^{177,182-185}$

In order to improve the mechanical properties of the biomimetic copolymers that were presented in chapter 3 via additional crosslinking, three different strategies will be presented in the next sections. These include the preparation of nanocomposite materials using silica nanoparticles and the introduced biomimetic multiblock copolymers, and switching of the macromolecular architecture from multiblock copolymers to diblock star copolymers containing hydrogen bonding monomers. Additionally, a novel mixing approach is discussed that provides polymers with additional crosslinking through the redistribution mechanism (see section 2.2) of polyfunctional RAFT agents. That is, when mixtures of polyfunctional and star RAFT agents are applied in the polymerization process, the star-shaped topological features are implemented into the polymeric material thereby altering the mechanical properties. 


\subsection{Addition of silica nanoparticles to biomimetic multiblock copolymers ${ }^{2}$}

When inorganic particles are added to a polymer matrix, a multitude of effects may occur that influence the mechanical properties of the resulting composite material, as already described in the preface of this chapter. Though the fundamental aspects of the interactions between inorganic particles and polymers are still topic of current research, ${ }^{186}$ the addition of, for instance, carbon black, zinc oxide or magnesium sulfate particles was already used in the beginning of the $20^{\text {th }}$ century to improve the mechanical properties of car tires, such as rolling friction. ${ }^{187}$

The strengthening of polymers via inorganic filler materials is very attractive for potential applications as the resulting composite materials exhibit a relatively low density, and yield lightweight materials. ${ }^{188}$ To retain this advantage, the content of inorganic filler material has to be kept low and, therefore, the effect of the filler material on the mechanical properties of the composite materials should be maximized. In this respect, nanoparticles have proven to be highly efficient due to their larger specific surface area per volume compared to, for instance, micron sized particles and the resulting stronger interactions between particles and polymer matrix. ${ }^{176-178,189-191}$ Consequently, the resulting composite materials are also termed nanocom-posites. However, the higher surface energy simultaneously results in a ten-dency of the nanoparticles to form agglomerates, thereby negating the dimensional advantage. ${ }^{176,177,187}$

In order to overcome this inherent limitation, nanoparticles may be functionalized with polymer to screen the particle cores and reduce their mutual attraction, hence avoiding agglomeration. 174,177,192 Preferably, the particles are functionalized with the polymer being chemically identical to the matrix polymer in the composite material to ensure favourable enthalpic interactions of the components. ${ }^{193}$ The wetting of the grafted polymer brushes by the matrix polymer still imposes a confinement on the polymer chains, leading to a loss of configurational entropy and hence to possible demixing of grafted particles and the matrix. ${ }^{194}$ Two parameters may suppress this phenomenon called autophobic dewetting, which are the grafing density and the molar mass of the grafted polymer with respect to the matrix polymer. Either reducing the

\footnotetext{
2 The results that are reported in this section are part of the bachelor thesis „Synthese von BlockCopolymeren unter Zusatz von polymerfunktionalisierten Silica-Nanopartikeln via RAFT-Polymerisation und Untersuchung der mechanischen Eigenschaften“ by Lennart Reuter.
} 

crosslinking

grafting density or reducing the molar mass of the matrix polymer may promote mixing of particles and matrix. ${ }^{194}$ The former shows a lower limit as the particle cores still need to be shielded sufficiently ${ }^{175}$ and the latter is limited due to practical reasons as the mechanical performance deteriorates with decreasing molar mass. ${ }^{55}$ To overcome these inherent limiations, so called bimodal brushes were developed, covering the nanoparticles with polymers of different chain lengths. Lately, this field has shown growing interest due to the accessibility of such modified particles via RAFT polymerization. ${ }^{183,185,195}$ As already outlined in the preface of this chapter, the impact of nanoparticles on the mechanical properties of nanocomposites may be most pronounced when the particles form superstructures such as percolation networks. Bimodal brushes promote the formation of such structures and give rise to additional structures compared with nanoparticles that are covered with monomodal brushes. ${ }^{196,197}$ Additionally, the dispersion of nanoparticles may be improved when grafting bimodal brushes rendering this approach a promising field of future research in nanocomposite materials. 195

In this work it was aimed to improve the mechanical properties of the presented biomimetic multiblock copolymers (see chapter 3 ) via the addition of unmodified and polymer grafted silica nanoparticles. Silica nanoparticles are an excellent choice as they are inodorous, chemically inert, biocompatible and relatively inexpensive. ${ }^{198}$ Also, they may be easily synthesized with control over the resulting particle size distrubtion via the Stöber process. ${ }^{199,200}$ To assure compatibility of the nanoparticles and the copolymer matrix, APA and MA monomer are used to prepare polymer-grafted nanoparticles. Hereafter, the synthesis and characterization of the respective materials, that is, the unmodified and polymer-grafted nanoparticles and the biomimetic multiblock copolymer is presented. Subsequently, the influence of the nanoparticles on the mechanical properties is elucidated by investigations into the neat multiblock copolymer and the respective nanocomposite materials via tensile testing and differential scanning calorimetry.

\subsubsection{Synthesis}

For the preparation of polymer-grafted nanoparticles, the graftingto, grafting-from and grafting-through approach may be distinguished, each offering distinct advantages and challenges. ${ }^{187,201}$ All methods may involve the use of reversible-deactivation radical polymerization (RDRP) 
techniques. As RAFT polymerization was the RDRP technique of choice in this work, corresponding advantages will be highlighted in the following.

The grafting-to approach utilizes presynthesized polymer chains being subsequently tethered to the particle surface. Using a controlled or living polymerization technique, the surface may be grafted with welldefined polymer chains. If the polymer was produced via RAFT and the anchor group constitutes the Z-group, only chains carrying an end-group functionality are bound to the surface and may hence be utilized in, for instance, a subsequent copolymerization. This method usually yields lower grafting density compared to the grafting-from or graftingthrough approach. ${ }^{201}$ The grafting process, however, is not only limited to chemical bonding for RAFT functionalized polymers but also physisorption of, for example, the RAFT group to gold surfaces is a wellestablished method. ${ }^{76-79}$ The grafting-through method utilizes molecules carrying vinylic groups that are bound to the particle surface. Afterwards, the particles may be used in a controlled polymerization to obtain uniform polymer chains on the particle surface.202

The grafting-from approach is arguably the most utilized method to produce polymer-grafted nanoparticles.202 Controlling agents are tethered to the particle surface and allow for controlled chain growth from the surface. With RAFT, two different approaches may be followed by anchoring the RAFT agent either via its R- or Z group. When the Rgroup approach is applied, the growing macromolecular chains are located on the particle and a very high grafting density may be realized. In the Z-group approach, which is unique to RAFT polymerization, RAFT agents are linked to the particles through their stabilizing Z-groups. The Z-group approach is schematically shown in Scheme 4-1. As Scheme 4-1 illustrates, the RAFT groups are always connected to the particle surface and polymer chain growth can only occur in solution. Since radical species need to diffuse to the surface and then react with the RAFT agent to form actual polymer brushes the technique is also referred to as grafting-to. ${ }^{201,203}$ This direct exchange of grafted polymer and polymer in solution leads to very uniform chain lengths of free and tethered polymer species. Due to this feature the Z-group approach was the method of choice in the present work. In both, R- and Z-group approach, sacrificial RAFT agent may be added to enhance the control of the polymerization and facilitate polymer characterization. ${ }^{203,204}$ 

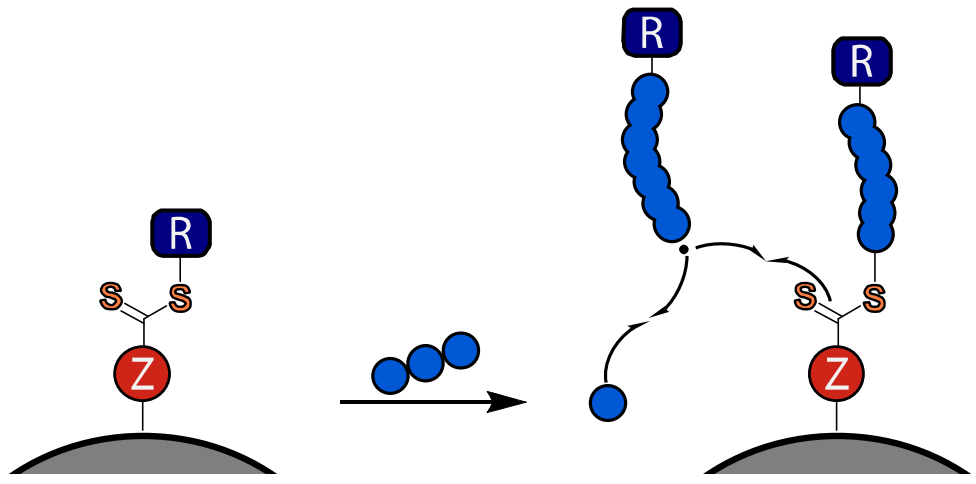

Scheme 4-1 Schematic illustration of the grafting-from approach using a RAFT group that is anchored via its Z-group.

\subsubsection{RAFT immobilized silica nanoparticles}

Silica nanoparticles were prepared in this work following the Stöber process that allows for the synthesis using tetraethyl orthosilicate in methanol, ethanol and water under the addition of ammonia (see Scheme 4-2). ${ }^{199}$ Choosing the solvent carefully, the size of the particles may be tailored as sterically more hindered alcohols lead to larger nanoparticles. ${ }^{200}$
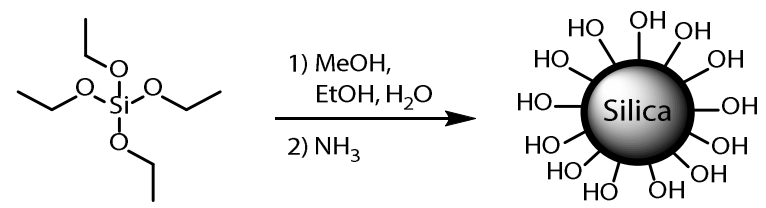

Scheme 4-2 Reaction scheme for the synthesis of silica nanoparticles. Note, that the drawing does not represent the true size ratios of the components.

Thermogravimetric analysis (TGA) of the particles (see Figure 4-2) shows a relative mass loss of $12.5 \%$ which is caused by residual solvent. Afterwards the nanoparticles were immobilized with the RAFT agent BD2PT (see Figure 4-3) which was kindly provided by Dennis Hübner. The RAFT agent contains a dimethoxysilane anchor group that mediates the immobilization reaction. Since the RAFT agent should be used for the polymerization of APA and MA, careful choice of the leaving group was necessary for an effective reinitiation by the RAFT agent. The benzyl group may be assumed to be a reasonable choice as it has been shown in 
literature that $\mathrm{APA}^{119}$ and $\mathrm{MA}^{205}$ poly-merizations can be mediated in a controlled fashion. The functionalized nanoparticles were investigated via TGA and exhibited a larger mass loss than the unfunctionalized particles (see Figure 4-2). Though residual solvent may also contribute to the overall mass loss, the measurement still suggests a successful immobilization of the RAFT agent. The functionalized nanoparticles were subsequently used for the preparation of poly-APA and poly-MA grafted nanoparticles (see section 4.2.1.3).

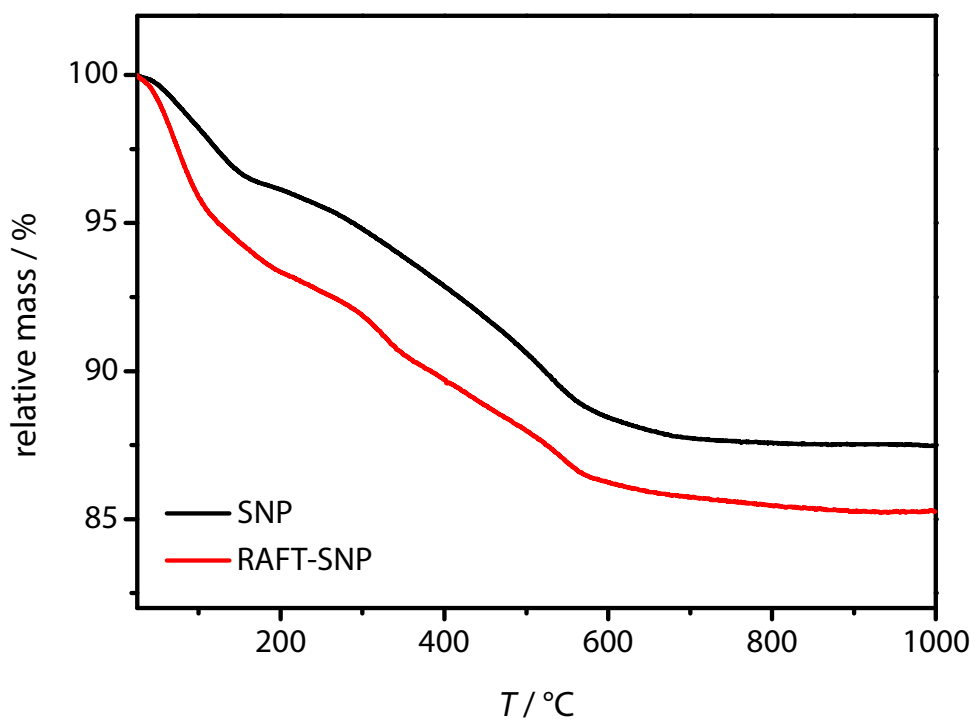

Figure 4-2 Comparison of the thermogravimetric analysis curves of unfunctionalized (SNP) and RAFT functionalized (RAFT-SNP) silica nanoparticles.<smiles>CO[Si](C)(CCCSC(=S)SCc1ccccc1)OC</smiles>

\section{BD2PT}

Figure 4-3 RAFT agent BD2PT carrying a dimethoxysilane anchor. The RAFT agent was kindly provided by Dennis Hübner. 


\subsubsection{Synthesis of matrix multiblock copolymer poly(APA- block-methyl acrylate)}

For the studies into the nanocomposite systems, a new batch of biomimetic multiblock copolymer was prepared in order to provide the same copolymer matrix for all systems. Therefore, the synthesis of polyfunctional RAFT agent poly-DMATC was also repeated. The results of all reactions are listed in Table 4-1. A slightly lower molar mass was obtained for poly-DMATC compared to the original study (see chapter 3 ), which corresponds to an average number of TTC groups of 6.6 per molecule. Afterwards, homopolymerization of APA was conducted to obtain multiblock polymer APAMB. In accordance with the results discussed earlier (see section 3.2.2) a relatively low apparent molar mass of the multiblock homopolymer was determined which indicates incomplete methylation of the carboxylic acid groups of APA prior to SEC characterization. However, aiming at a certain target degree of polymerization is not required as the same polymer would be used for all following studies.

In the next step, the copolymerization with MA was conducted to obtain the matrix polymer APA-MAMB. Due to the large scale of the reaction (35 g of MA in $120 \mathrm{~mL}$ 1,4-dioxane) it was decided to prepare a stock solution, which was then degassed and distributed among multiple $5 \mathrm{~mL}$ polymerization vials under argon. Subsequently, the polymerization vials were put into a thermostated heating block. This way, optimum heat transfer was assured for all samples, as conducting the polymerization on such a scale in a flask may lead to problems with regard to the control of the polymerization (see section 4.3.1.2). Afterwards, the reaction mixtures of all polymerization vials were combined except one and SEC was measured for the dried polymers. SEC analysis yields almost identical results for the polymer samples (see Table 4-1), verifying the correctness of the approach. In Figure 4-4 the $\mathrm{SE}$ chromatograms of the precursor $\mathrm{APA} \mathrm{MB}^{\mathrm{B}}$ and the APA-MA ${ }^{\mathrm{MB}}$ copolymer samples are shown. The SE chromatograms clearly demonstrate the significant increase in molar mass of the copolymers compared to the homopolymer without any low molar mass tailing, veryfing the successful copolymerization procedure. The prepared APA-MAMB copolymer was investigated by tensile testing to obtain reference values for the nanocomposite materials. Afterwards, polymer-grafted nanoparticles were prepared using APA and MA. The results are presented in the following section. 
Table 4-1 Results of the SEC analysis of the prepared polyfunctional RAFT agent polyDMATC, the homopolymer APAMB and the copolymer APA-MAMB. The latter was prepared by dividing a stock solution into multiple polymerization vials (mixture), combining all samples except for one afterwards (single sample). For further explanations refer to the text. SEC was measured versus linear polystyrene standards. Corresponding SEC curves are depicted in Figure 4-4. The number average molar mass of poly-DMATC corresponds to an average of 6.6 TTC groups per molecule.

\begin{tabular}{ccc}
\hline \hline sample & $\begin{array}{c}\bar{M}_{\mathrm{n}, \mathrm{SEC}} / \\
10^{4} \mathrm{~g} \mathrm{~mol}^{-1}\end{array}$ & $Ð$ \\
\hline poly-DMATC & 0.24 & 2.1 \\
APA $^{\mathrm{MB}}$ & 0.56 & 1.5 \\
$\begin{array}{c}\text { APA-MA } \\
\text { (single sample) }\end{array}$ & 4.3 & 2.2 \\
$\begin{array}{c}\text { APA-MA } \\
\text { (mixture) }\end{array}$ & 4.1 & 2.2 \\
\hline \hline
\end{tabular}

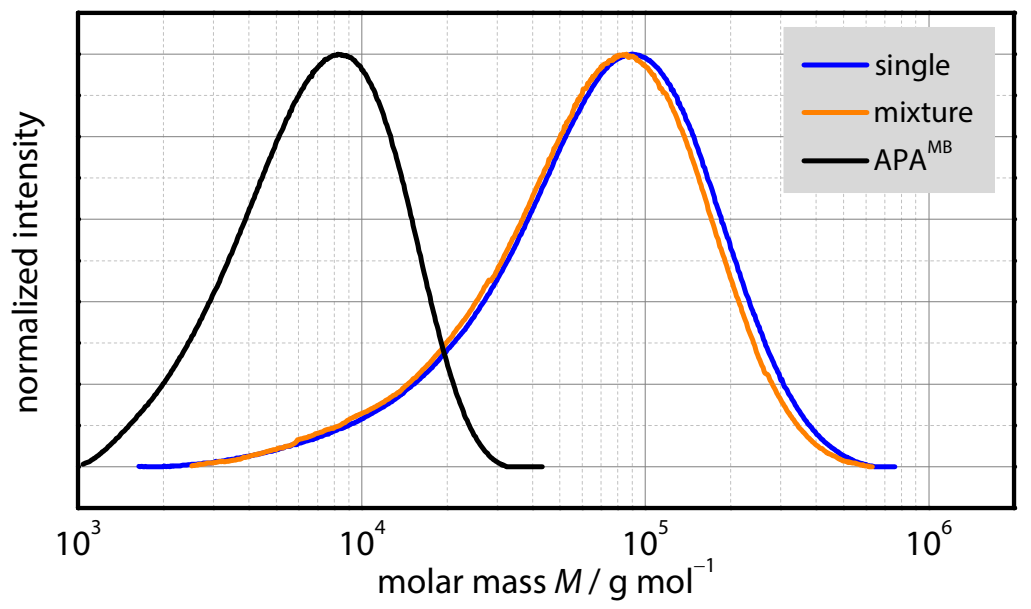

Figure 4-4 SE chromatograms (THF) of the homopolymer sample APAMB and copolymer sample APA-MAMB as recorded by RI detection. Copolymerization was conducted using a stock solution of macro-RAFT agent, initiator, MA and solvent, which was divided into multiple polymerization vials. The blue SEC trace corresponds to the copolymer material obtained from a single polymerization vial, the orange SEC trace was obtained for a copolymer material that combined all residual polymerization vials. 


\subsubsection{Synthesis of polymer-grafted nanoparticles}

In this work, polymer-grafted silica nanoparticles (SNPs) were prepared by the grafting-from approach using BD2PT as RAFT agent (see Figure 4-3) which is anchored via its Z-group. It was decided to synthesize poly-APA and poly-MA grafted nanoparticles as both may provide distinct advantages through either hydrogen bonding interactions with the matrix polymer in the former, or entanglement formation in the latter case. To improve the degree of control over the polymerizations, sacrificial RAFT agent was added during both polymerizations.

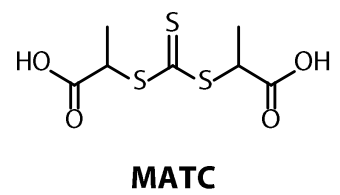

Figure 4-5 Bifunctional RAFT agent MATC.

Starting with the preparation of poly-MA grafted (MA-SNP) nanoparticles, DMATC was added as sacrificial RAFT agent. Due to the assumed uniformity of grafted polymer chains and chains in solution in the Z-group approach, free polymer chains were analysed via SEC to deduce the molar mass of the grafted chains. It has to be noted that DMATC is a bifunctional RAFT agent which should consequently yield polymer chains of about twice the length of the grafted chains. Therefore, determined molar masses need to be divided by a factor of 2 to obtain the actual molar mass of the grafted chains, assuming comparable polymerization kinetics. For the grafting of poly-APA, MATC (see Figure 4-5) was added as sacrificial RAFT agent. In preliminary experiments it was determined that MATC can yield APA homopolymer with lower dispersities than DMATC. The results of the SEC analysis of the free polymer are collated in Table 4-2. For both types of nanoparticles, the formation of polymer with good control is observed.

Investigation of the polymer-grafted nanoparticles via TGA was conducted to obtain the polymer loading. The results are shown in Figure 4-6. Both, poly-MA (MA-SNP) and poly-APA (APA-SNP) grafted nanoparticles exhibit a larger mass loss than the RAFT immobilized particles, corresponding to net loadings of $5.7 \%$ (MA-SNPs) and $4.2 \%$ (APA-SNPs). 
Table 4-2 Results of the SEC analysis of polymer that is formed in solution during the polymerization process in the presence of RAFT functionalized nanoparticles. The corrected number average molar mass is obtained by dividing the determined number average molar mass by a factor of two as free bifunctional RAFT agent was added. SEC was measured versus linear polystyrene standards (APA-SNP) or linear poly(methyl acrylate) standards (MA-SNP).

\begin{tabular}{|c|c|c|c|}
\hline sample & $\begin{array}{c}\bar{M}_{\mathrm{n}, \mathrm{SEC}} / \\
10^{3} \mathrm{~g} \mathrm{~mol}^{-1}\end{array}$ & $\begin{array}{c}\bar{M}_{\mathrm{n}, \mathrm{corr}} / \\
10^{3} \mathrm{~g} \mathrm{~mol}^{-1}\end{array}$ & $D$ \\
\hline MA-SNP & 11.5 & 5.6 & 1.1 \\
\hline APA-SNP & 5.7 & 2.9 & 1.3 \\
\hline
\end{tabular}

To give additional proof of successful polymer immobilization on the nanoparticles surfaces, all prepared nanoparticle samples, that is, unfunctionalized, RAFT functionalized, poly-MA grafted and poly-APA grafted silica nanoparticles were characterized via dynamic light scattering (DLS). DLS allows to determine the size of particles in solution by measuring their time-dependent change in scattering intensity. 206 More precisely, the standard deviation of the average scattering intensity is measured which can then be related to the diffusion coefficient $D$. The diffusion coefficient is linked to the hydrodynamic radius via the Stokes-Einstein equation:

$$
D=\frac{k_{\mathrm{B}} T}{6 \pi \eta R_{\mathrm{h}}} .
$$

The diffusion coefficient therefore depends on temperature $T$, viscosity $\eta$ and hydrodynamic radius $R_{\mathrm{h}}$ of the particles. Thus, DLS does not give quantitative information about the particle size but differences in hydrodynamic radius may certainly be compared

Results of the DLS measurements are depicted in Figure 4-7. After immobilization of RAFT, the maximum of the intensity distribution is shifted to smaller particle sizes and the intensity distribution also becomes more narrow compared to unfunctionalized silica nanoparticles. After immobilization of either poly-MA or poly-APA, further narrowing of the intensitity distributions is observed, indicating the successful immobilization of polymer chains on the particle surfaces. Therefore, the combination of SEC, TGA and DLS measurements suggests that the nanoparticles were successfully immobilized with polymer. In the following section, the influence of the unfunctionalized and polymergrafted SNPs is elucidated by tensile testing and DSC measurements. 
4 Tuning the mechanical properties of biomimetic copolymers via crosslinking

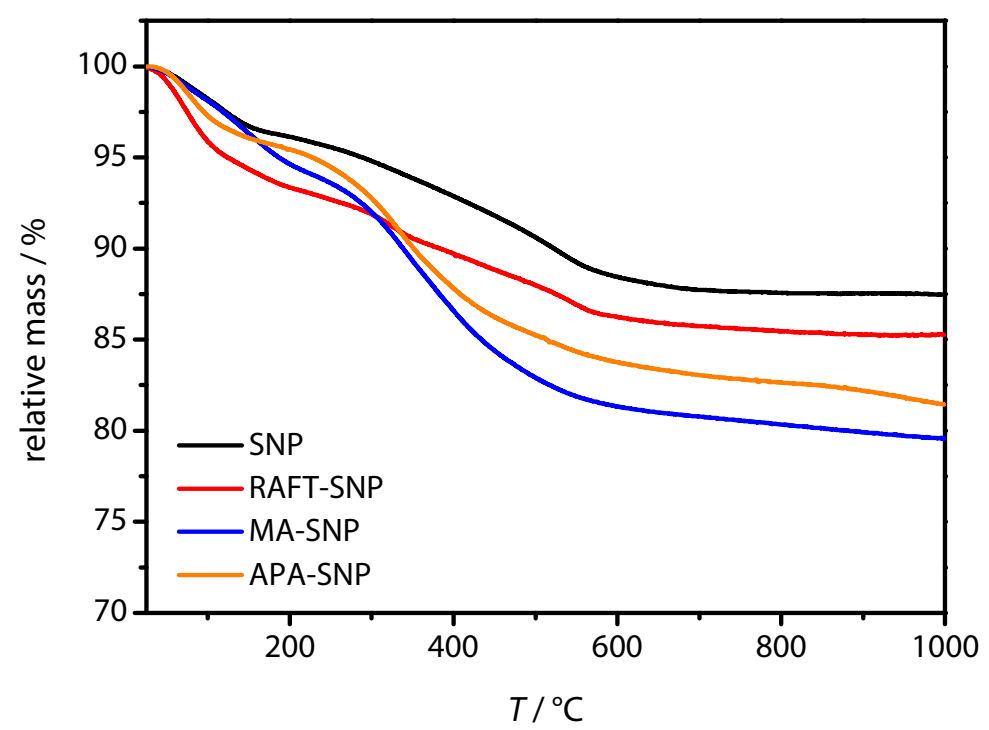

Figure 4-6 Comparison of the thermogravimetric analysis curves of unfunctionalized (SNP), RAFT functionalized (RAFT-SNP), poly-MA grafted (MA-SNP) and poly-APA grafted (APA-SNP) silica nanoparticles. 


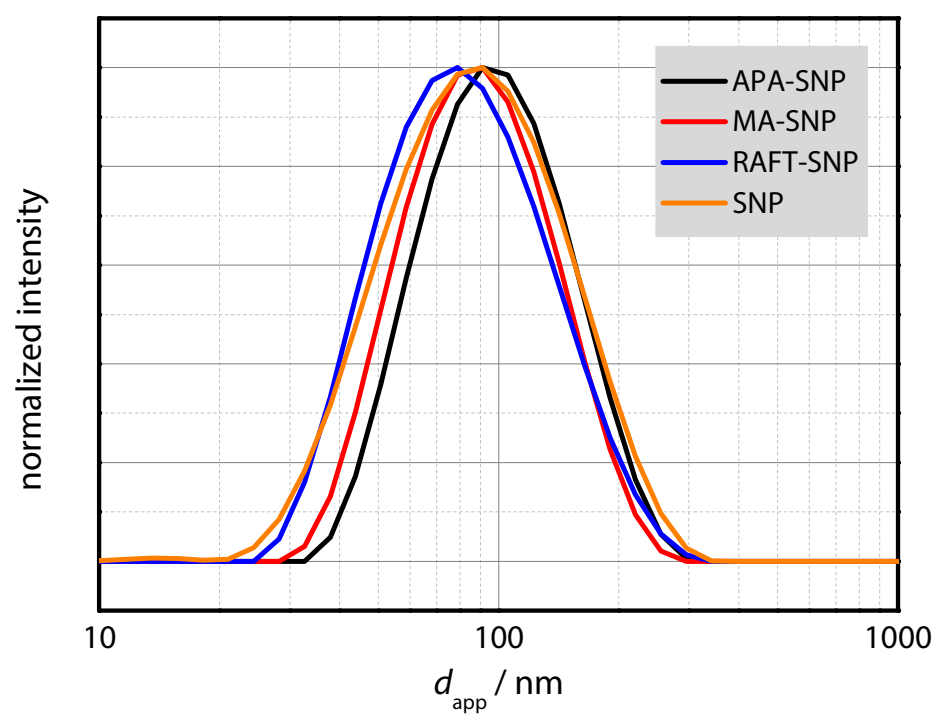

Figure 4-7 Normalized intensity distributions of the unfunctionalized (SNP), RAFT functionalized (RAFT-SNP), poly-MA grafted (MA-SNP) and poly-APA grafted (APASNP) silica nanoparticles in PGMEA versus the apparent particle diameter $d_{\mathrm{app}}$.

\subsubsection{Tensile testing}

In this section, the tensile testing results of the pure matrix copolymer and the corresponding nanocomposites are presented. Starting with the matrix copolymer, the effect of unfunctionalized silica nanoparticles on the mechanical properties of the nanocomposites is shown first and afterwards the influence of the polymer-grafted nanoparticles on the mechanical properties is elucidated. To facilitate the discussion of the obtained results, the corresponding results of DSC measurements of the nanocomposites and the matrix polymer are given in each case. It has to be noted that the results presented here were obtained during the summer of 2015. At that point, no temperature control was present for the tensile testing machine and, therefore, experiments had to be conducted at different temperatures. It is wellknown, that the mechanical properties of not only polymers ${ }^{84,138}$ but also polymer nanocomposites 189,207 significantly depend on temperature. Additionally, the glass transition temperature of poly-MA is close to room temperature $\left(T_{\mathrm{g}} \text { (poly-MA) } \approx 10^{\circ} \mathrm{C}\right)^{102}$ and a great influence on 

crosslinking

mechanical properties is hence expected. Thus, interpretation and generalization of experimental findings is limited. However, results of tensile testing experiments will be given with corresponding temperatures and the experimental findings are discussed qualitatively.

For the preparation of tensile specimens, the drop-casting procedure was used in accordance with the biomimetic copolymers discussed earlier (see section 3.3), to ensure comparability of the results. PGMEA was also used as solvent, as it dissolves the copolymer and is capable of dispersing the silica nanoparticles well. The latter was tested via DLS (see Figure 4-7) and only a monomodal intensity distribution was obtained, indicating the lack of aggregates. The nanocomposites were prepared by slowly mixing a concentrated polymer solution with a dilute solution of dispersed nanoparticles and subsequent drop-casting.

Table 4-3 Mechanical properties of three tensile specimens of the matrix copolymer APA-MAMB. The respective stress-strain curves are depicted in Figure 4-8. For each sample the corresponding ambient temperature is indicated.

\begin{tabular}{lcll}
\hline \hline sample & $\varepsilon_{\text {br }} / \%$ & $\sigma_{\text {UTS }} / \mathrm{MPa}$ & $U_{\mathrm{T}} / \mathrm{MPa}$ \\
\hline $\mathrm{I}\left(20.9^{\circ} \mathrm{C}\right)$ & 675 & 0.65 & 2.94 \\
II $\left(25.5^{\circ} \mathrm{C}\right)$ & 1970 & - & 1.61 \\
III $\left(25.5^{\circ} \mathrm{C}\right)$ & 1503 & 0.04 & 1.56 \\
\hline \hline
\end{tabular}




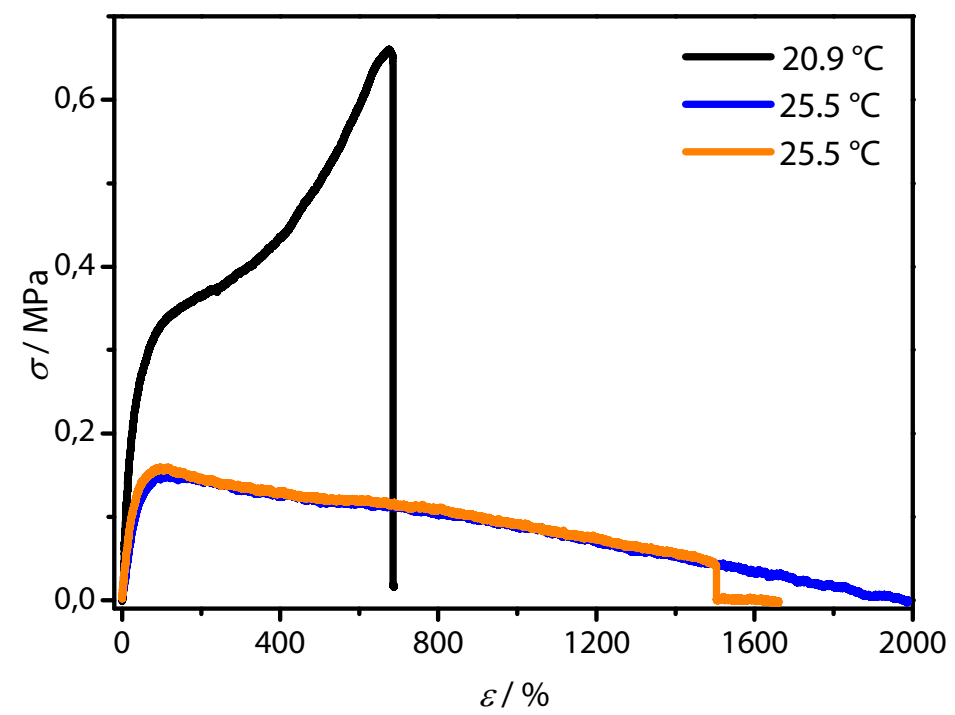

Figure 4-8 Stress-strain curves of the matrix copolymer APA-MAMB. Respective ambient temperatures are added to the diagram. The glass transition temperature of the copolymer was determined to be $13^{\circ} \mathrm{C}$.

\section{Matrix copolymer APA-MAMB}

The stress-strain curves of the matrix copolymer and the corresponding mechanical quantities are shown in Figure 4-8 and Table 4-3. Two distinct stress-strain curves result depending on the ambient temperature. At $20.9^{\circ} \mathrm{C}$ the polymer shows a tough behaviour and an increase of stress, that is, strain hardening is observed before the sample fails at an elongation of $675 \%$. At elevated temperature, however, the polymer exhibits creeping and a constant decrease of stress before the samples fail. The first curve proves the incorporation of poly-APA domains that lead to a sample toughening compared to pure poly-MA (also compare section 3.3). At $25.5^{\circ} \mathrm{C}$ the influence of strengthening poly-APA segments vanishes as the polymer is stressed well above its glass transition temperature $\left(T_{\mathrm{g}}=13^{\circ} \mathrm{C}\right)$. Compared to the biomimetic copolymers discussed above (see chapter 3 ), a smaller tensile strength and a larger elongation at break are observed, which indicates a smaller content of APA in the copolymers. Hereafter the influence of unfunctionalized silica nanoparticles on the mechanical properties of the matrix copolymer is elucidated. 

crosslinking

\section{Unfunctionalized SNPs}

For the preparation of silica nanocomposites it was decided to investigate three different contents of unfunctionalized SNPs. In literature, silica particles are used as the minor component of nanocomposites and weight contents around $10 \%$ are conventionally applied.176,177,208,209 Even though larger contents have been investigated as well, the inherent trade-off of higher density and weight of the resulting compounds renders them impractical for applications. Additionally, properties such as elongation at break and tensile strength may show a maximum at nanoparticle contents of about $10 \mathrm{wt} \%$, which may be the result of aggregation at higher weight fractions. ${ }^{176}$ Though such observations are dependent on the polymer matrix and other factors, such as processing conditions, it was decided to investigate contents of 1,5 and $10 \mathrm{wt} \%$ SNPs to avoid aggregation phenomena that would cause deterioration of mechanical properties.

It was attempted to investigate at least three different tensile specimens for each composition. For a content of $1 \mathrm{wt} \%$ it was not possible to prepare three specimens as the third one was prone to void formation during the drop-casting process and an optically homogeneous specimen could not be obtained. The stress-strain curves for the investigated specimens are depicted in Figure 4-10a and respective mechanical properties are collated in Table 4-4. Both stress-strain curves display a ductile behaviour of the specimens and an increase of stress before sample failure. This indicates an effective strengthening and crosslink formation by the nanoparticles. At $23.3^{\circ} \mathrm{C}$ a lower stress level is observed with a simultaneous increase in elongation at break. This is presumably due to the higher chain mobility which attenuates the strengthening via the nanoparticles. Going to $5 \mathrm{wt} \%$ of SNPs (see Figure 4-10b) even higher tensile strengths are observed and the temperature dependence of the mechanical properties seems to be less pronounced. Between 21.2 and $24.0^{\circ} \mathrm{C}$ comparable elongations at break are observed, only at $25.3^{\circ} \mathrm{C}$ the elongation at break increases and the tensile strength decreases. The higher mobility of polymer chains is likely to explain this observation. At $10 \mathrm{wt} \%$ content of SNPs (see Figure 4-10c) a decrease of tensile strength and an increase in elongation at break is observed compared to the nanocomposites with $5 \mathrm{wt} \%$ of SNPs. Additionally, the second sample that was measured at $23.3^{\circ} \mathrm{C}$ (blue curve in Figure 4-10c) displayed a stress-strain behaviour that resembled the pure matrix copolymer at $25.5^{\circ} \mathrm{C}$ (see Figure 4-8). It has to be noted, that the tensile specimen was stored at room temperature 
for $36 \mathrm{~h}$ prior to tensile testing. One may assume that an agglomeration of SNPs has occured within that time frame which could ultimately cause a demixing of the components.

To check this assumption, several tensile specimens were investigated via DSC before and after tensile testing. The tensile testing had no influence on the glass transition temperature. However, for sample II with $10 \mathrm{wt} \%$ SNPs in comparison to samples with smaller SNP content a slight reduction of the glass transition temperature is observed, which coincides with the decreased tensile strength and increased elongation at break as the sample appears to resemble the pure matrix copolymer. This would confirm the suggested agglomeration and demixing of the nanoparticles. Other than that, the weight content of SNPs does not seem to influence the glass transition temperature but it is increased compared to the pure matrix copolymer $\left(T_{\mathrm{g}}=13^{\circ} \mathrm{C}\right)$. An effective reinforcement by the nanoparticles and interaction with the matrix polymer may, therefore, also be suggested based on the DSC measurements. ${ }^{195}$ An additional error source of the presented tensile tests concerns the drop-casting process. With increasing weight content of SNPs the samples were more prone to bubble formation which led to the formation of voids when the bubbles ruptured. These voids were then refilled with nanocomposite solution and, thus, an inhomogeneous distribution of SNPs might result.

During all tensile tests of the nanocomposites, strain whitening of the specimens could be observed after an elongation of about $100 \%$. Strain whitening was also observed for the biomimetic copolymers discussed in chapter 3. While the formation of crazes is the predominant mechanism for strain whitening of polymers, ${ }^{142,143}$ the formation of cavities causes strain whitening in composite materials. ${ }^{141,210}$ In clay nanocomposite materials, that is, layered silicates, a reduced susceptibility to strain whitening was reported. ${ }^{141}$ However, the system presented herein seems to show promoted strain whitening due to the filler material as the whitening is only partially reversible after the release of stress (see Figure 4-9). Yet, for the biomimetic copolymers the whitening was completely reversible (see section 3.3). 
4 Tuning the mechanical properties of biomimetic copolymers via crosslinking

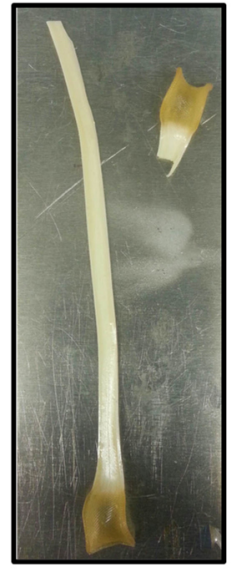

$0 \min$

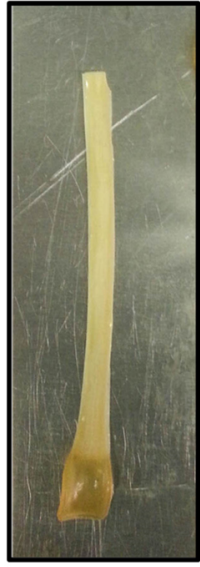

$5 \mathrm{~min}$

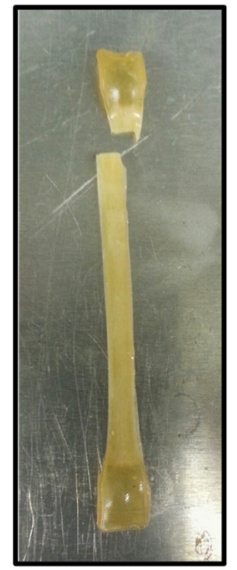

$25 \mathrm{~min}$

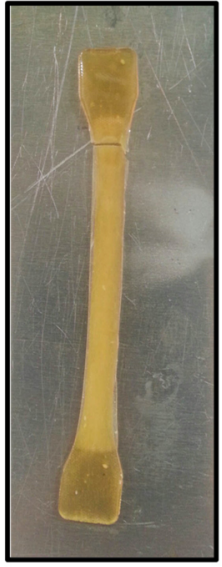

$18 \mathrm{~h}$

Figure 4-9 Reversibility of strain whitening after sample failure. Given are the time intervals after sample failure.

Comparing the tensile testing of the nanocomposites with unfunctionalized SNPs to the pure matrix copolymer, a significant reinforcement and toughening of the samples is observed. These results suggest effective crosslink formation through the nanoparticles. These might be mediated by the hydroxyl functionalities of the silica particle surfaces via the formation of hydrogen bonds to the poly-APA domains of the copolymer. Such a formation of hydrogen bonds has been reported in literature for polyvinylpyrrolidone ${ }^{211}$ and modified polybutadiene ${ }^{212}$ matrix polymers. With regard to the presented results, a content of $5 \mathrm{wt} \%$ SNPs displayed the largest tensile strength and toughness values. However, due to the limited number of samples and lack of control over the temperature conditions one may not conclusively deduce $5 \mathrm{wt} \%$ as an optimum content of unfunctionalized SNPs. 


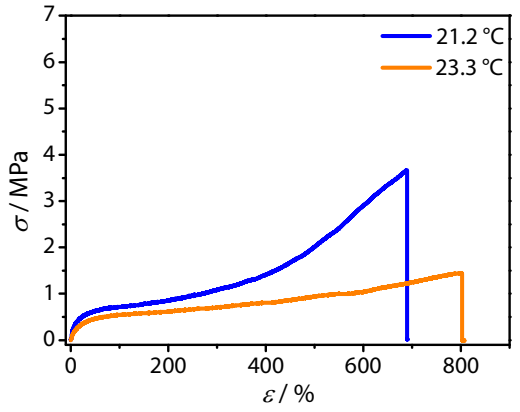

a) $1 \mathrm{wt} \%$

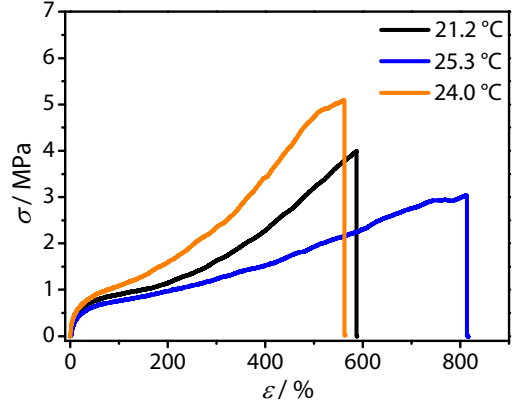

b) $5 \mathrm{wt} \%$

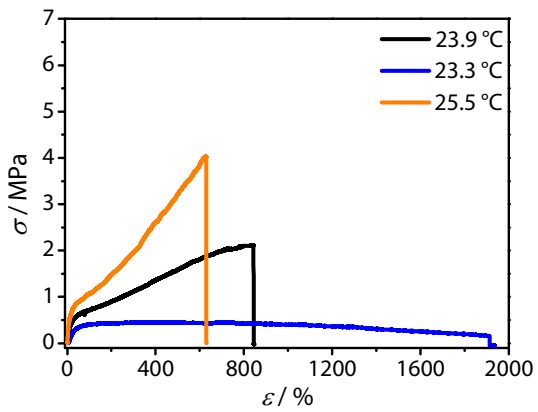

c) $10 \mathrm{wt} \%$

Figure 4-10 Stress-strain curves for nanocomposites of matrix copolymer APA-MAMB and unfunctionalized SNPs at three different weight contents of SNPs. The corresponding mechanical properties are collated in Table 4-4. 
4 Tuning the mechanical properties of biomimetic copolymers via crosslinking

Table 4-4 Mechanical properties of nanocomposites of the matrix copolymer APA$M \mathrm{~A}^{\mathrm{MB}}$ and three different weight contents of unfunctionalized silica nanoparticles. The respective stress-strain curves are depicted in Figure 4-10.

\begin{tabular}{lccc}
\hline \hline \multicolumn{1}{c}{ sample } & $\varepsilon_{\mathrm{br}} / \%$ & $\sigma_{\mathrm{UTS}} / \mathrm{MPa}$ & $U_{\mathrm{T}} / \mathrm{MPa}$ \\
\hline I wt\% & & & \\
I $\left(21.2^{\circ} \mathrm{C}\right)$ & 690 & 3.7 & 10.6 \\
II $\left(23.3^{\circ} \mathrm{C}\right)$ & 800 & 1.4 & 6.8 \\
\hline $5 \mathrm{wt} \%$ & & & \\
I $\left(21.2^{\circ} \mathrm{C}\right)$ & 587 & 4.0 & 10.9 \\
II $\left(25.3^{\circ} \mathrm{C}\right)$ & 813 & 3.0 & 13.6 \\
III $\left(24.0^{\circ} \mathrm{C}\right)$ & 561 & 5.1 & 14.1 \\
\hline $10 \mathrm{wt} \%$ & & & \\
I $\left(23.9^{\circ} \mathrm{C}\right)$ & 843 & 2.1 & 11.5 \\
II $\left(23.3^{\circ} \mathrm{C}\right)$ & 1912 & 0.1 & 6.8 \\
III $\left(25.5^{\circ} \mathrm{C}\right)$ & 628 & 4.0 & 13.6 \\
\hline \hline
\end{tabular}

Table 4-5 Glass transition temperatures that were determined for the nanocomposites of matrix copolymer APA-MA ${ }^{\mathrm{MB}}$ and three different weight contents of unfunctionalized SNPs by means of DSC. Samples were chosen to aid in the interpretation of the tensile testing results.

\begin{tabular}{lc}
\hline \hline \multicolumn{1}{c}{ sample } & $T_{\mathrm{g}} /{ }^{\circ} \mathrm{C}$ \\
\hline $1 \mathrm{wt} \%$ & \\
I $\left(21.2^{\circ} \mathrm{C}\right)$ & 19 \\
II $\left(23.3^{\circ} \mathrm{C}\right)$ & 18 \\
\hline $5 \mathrm{wt} \%$ & \\
II $\left(25.3^{\circ} \mathrm{C}\right)$ & 18 \\
\hline $10 \mathrm{wt} \%$ & \\
II $\left(23.3^{\circ} \mathrm{C}\right)$ & 16 \\
III $\left(25.5^{\circ} \mathrm{C}\right)$ & 18 \\
\hline \hline
\end{tabular}




\section{Poly-MA functionalized SNPs}

After the investigation of nanocomposites with unfunctionalized SNPs, nanocomposites with polymer-grafted nanoparticles were prepared. Starting with poly-MA functionalized SNPs, composite materials with similar content of nanoparticles, that is, 1, 5 and $10 \mathrm{wt} \%$ were prepared. It is important to note that for the nanocomposite preparation the polymer-loading (5.7\% for poly-MA grafted SNPs) has to be taken into account. That is, for a given amount of nanocomposite fewer matrix polymer has to be added to keep the number of SNPs comparable to the nanocomposites with unfunctionalized SNPs. As outlined in the introduction of this chapter, the addition of polymergrafted nanoparticles should result in a more pronounced improvement of mechanical properties due to better mixing and more effective interactions with the polymer matrix. ${ }^{177}$

The obtained stress-strain curves are depicted in Figure 4-12 and the corresponding mechanical properties are collated in Table 4-6. After the addition of $1 \mathrm{wt} \%$ poly-MA grafted SNPs, similar stress-strain curves (see Figure 4-12a) compared to unfunctionalized SNPs are observed that result in comparable toughness values. The third sample, however, displays a significantly larger tensile strength that causes a more than two times larger sample toughness. One possible explanation is the lower ambient temperature and a different dispersion of the particles could also contribute to the observation. The latter argument is supported by DSC data, as the sample III exhibits a slightly higher glass transition temperature $\left(T_{\mathrm{g}}=19^{\circ} \mathrm{C}\right)$. At a content of $5 \mathrm{wt} \%$ functionalized SNPs it was only possible to produce two optically homogeneous tensile specimens. Similar to the unfun-ctionalized SNPs at $1 \mathrm{wt} \%$, the third specimen tended to form bubbles that could not be removed without void formation. The optically homogeneous specimens were tested via tensile testing and sample I exhibited a stress-strain behaviour similar to $1 \mathrm{wt} \%$ poly-MA grafted SNPs (see Figure 4-12b). The second specimen, despite lacking visible flaws, showed necking (see Figure 4-11 a) and consequently failed at significantly lower elongation. For the nanocomposites with $10 \mathrm{wt} \%$ poly-MA grafted SNPs it was possible to obtain three optically homogeneous specimens. The stress-strain curves appear to be different compared to the $1 \mathrm{wt} \%$ nanocomposites. The latter showed an initially lower level of stress and a relatively steep increase of stress before failure. At $10 \mathrm{wt} \%$ a constantly increasing stress is observed for all specimens after the elastic regimen. This suggests, that the deformation mechanism might change at the higher particle content. 
4 Tuning the mechanical properties of biomimetic copolymers via crosslinking

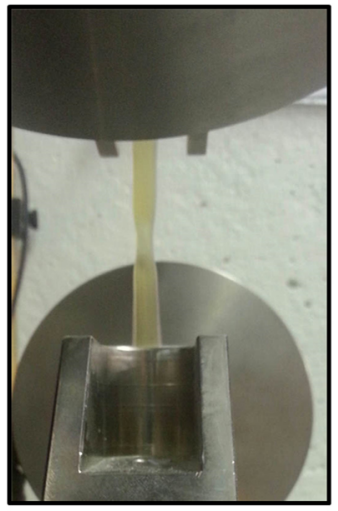

a)

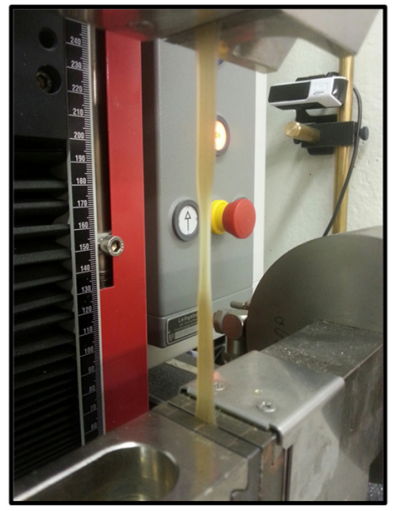

b)

Figure 4-11 a) Necking of sample II (orange curve in Figure 4-12 b) containing 5 wt\% of poly-MA functioalized SNPs, b) Necking of sample I (black curve in Figure 4-13 b) containing $5 \mathrm{wt} \%$ of poly-APA functionalized SNPs.

The third sample shows a lower tensile strength and elongation at break compared to the samples I and II (see Table 4-6). No temperature was recorded for that measurement, however, the weather record of the day of the experiment $(07 / 15 / 2015)$ states a maximum temperature of $25.7^{\circ} \mathrm{C}^{213}$ at that day. Therefore, the ambient temperature should be comparable among all the samples and poorer dispersion of particles in the specimen may explain the difference in stress-strain behaviour.

The observed strengthening of the samples compared to the pure copolymer is additionally supported by DSC measurements that show a significantly increased glass transition temperature (see Table 4-7). Furthermore, a particle content of $1 \mathrm{wt} \%$ yields significantly improved mechanical properties that are comparable to a particle content of $10 \mathrm{wt} \%$. However, at $5 \mathrm{wt} \%$ it was difficult to obtain homogeneous tensile specimen and one sample exhibited premature failure due to necking. 


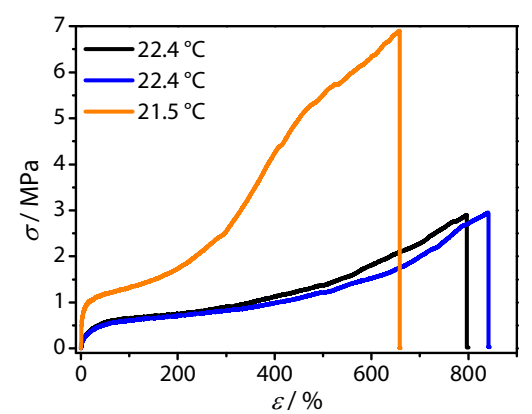

a) $1 \mathrm{wt} \%$

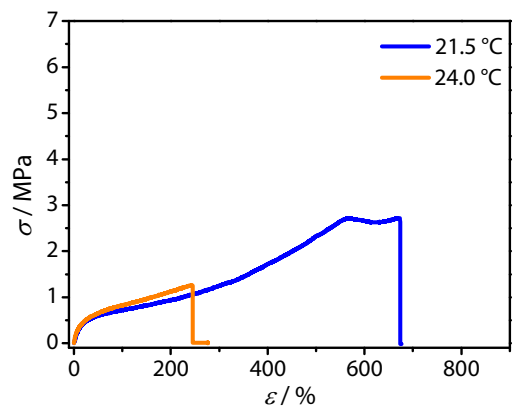

b) $5 \mathrm{wt} \%$

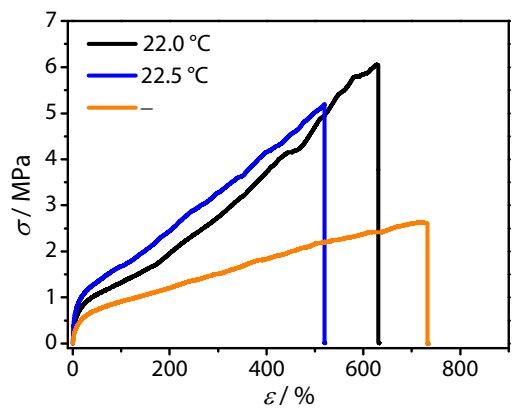

c) $10 \mathrm{wt} \%$

Figure 4-12 Stress-strain curves for nanocomposites of matrix copolymer APA-MAMB and poly-MA grafted SNPs at three different weight contents of SNPs. The corresponding mechanical properties are collated in Table 4-6. 
4 Tuning the mechanical properties of biomimetic copolymers via crosslinking

Table 4-6 Mechanical properties of nanocomposites of the matrix copolymer APA$\mathrm{MA}^{\mathrm{MB}}$ and three different weight contents of poly-MA grafted silica nanoparticles. The respective stress-strain curves are depicted in Figure 4-12.

\begin{tabular}{lccc}
\hline \hline \multicolumn{1}{c}{ sample } & $\varepsilon_{\text {br }} / \%$ & $\sigma_{\text {UTS }} / \mathrm{MPa}$ & $U_{\mathrm{T}} / \mathrm{MPa}$ \\
\hline 1 wt\% & & & \\
I $\left(22.4^{\circ} \mathrm{C}\right)$ & 797 & 2.9 & 10.4 \\
II $\left(22.4^{\circ} \mathrm{C}\right)$ & 841 & 3.0 & 10.5 \\
III $\left(21.5^{\circ} \mathrm{C}\right)$ & 658 & 6.9 & 22.8 \\
\hline $5 \mathrm{wt} \%$ & & & \\
I $\left(21.5^{\circ} \mathrm{C}\right)$ & 672 & 2.7 & 10.4 \\
II $\left(24.0^{\circ} \mathrm{C}\right)$ & 244 & 1.3 & 2.1 \\
\hline $10 \mathrm{wt} \%$ & & & \\
I $\left(22.4{ }^{\circ} \mathrm{C}\right)$ & 630 & 6.1 & 19.5 \\
II $\left(22.4^{\circ} \mathrm{C}\right)$ & 519 & 5.2 & 15.4 \\
III $($ see text) & 721 & 2.6 & 12.4 \\
\hline \hline
\end{tabular}

Table 4-7 Glass transition temperatures that were determined for the nanocomposites of matrix copolymer APA-MAMB and three different weight contents of poly-MA grafted SNPs by means of DSC. Samples were chosen to aid in the interpretation of the tensile testing results.

\begin{tabular}{lc}
\hline \multicolumn{1}{c}{ sample } & $T_{\mathrm{g}} /{ }^{\circ} \mathrm{C}$ \\
\hline $1 \mathrm{wt} \%$ & \\
I $\left(22.4^{\circ} \mathrm{C}\right)$ & 17 \\
III $\left(21.5^{\circ} \mathrm{C}\right)$ & 19 \\
\hline $5 \mathrm{wt} \%$ & \\
II $\left(22.0^{\circ} \mathrm{C}\right)$ & 16 \\
\hline $10 \mathrm{wt} \%$ & \\
II $\left(22.5^{\circ} \mathrm{C}\right)$ & 17 \\
\hline \hline
\end{tabular}




\section{Poly-APA functionalized SNPs}

After the investigation of the influence of poly-MA functionalized particles, which should preferably interact with poly-MA segments of the matrix copolymer, nanocomposites containing poly-APA functionalized particles were investigated. It may be envisioned that these particles interact via hydrogen bonding with poly-APA segments of the matrix and hence cause even more effective crosslinking.

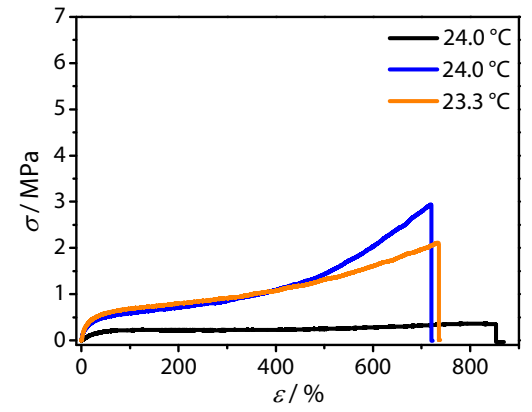

a) $1 \mathrm{wt} \%$

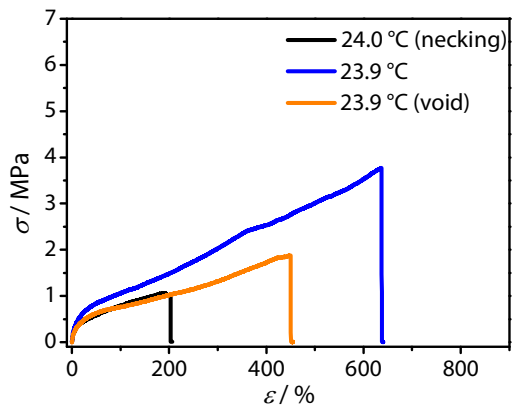

b) $5 \mathrm{wt} \%$

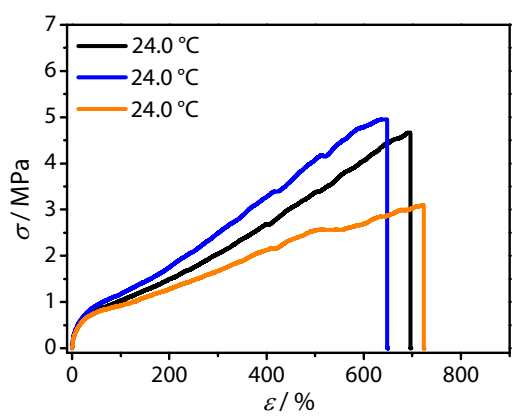

c) $10 \mathrm{wt} \%$

Figure 4-13 Stress-strain curves for nanocomposites of matrix copolymer APA-MAMB and poly-APA grafted SNPs at three different weight contents of SNPs. The corresponding mechanical properties are collated in Table 4-8.

The obtained stress-strain curves and corresponding mechanical quantities are shown in Figure 4-13 and Table 4-8. At a content of $1 \mathrm{wt} \%$, the poly-APA functionalized SNPs do not seem to influence the stress-strain behaviour as similar curves compared to the pure matrix copolymer are obtained (see Figure 4-13a). That is, after an initially almost constant stress, the stress shows a steeper increase before sample failure. The first sample (black curve) exhibits a distinct 

crosslinking

stress-strain curve. After the elastic region, no increase of stress is observed and the sample breaks after about $800 \%$ elongation. Since the sample did not display any visible flaws, an aggregation of particles or residual solvent, that would act as a plasticizer, might cause the observed stress-strain behaviour. Investigation of the sample via DSC yielded a glass transition temperature of $13^{\circ} \mathrm{C}$ which is similar to the pure matrix copolymer (see Table 4-9). This indicates particle aggregation and demixing, hence suppressing interaction of the particles with the matrix. Going to $5 \mathrm{wt} \%$ content of poly-APA functionalized SNPs a different stress-strain behaviour of the samples is observed (see Figure 4-13b). Sample II (blue curve) shows an almost linear increase of stress after the initial elastic regime, which was also observed for a content of $10 \mathrm{wt} \%$ poly-MA functionalized SNPs. Samples I and III (black and orange curves) displayed inhomogeneities that led to premature sample failure. Sample I showed necking (see Figure 4-11 b) and Sample III exhibited a void which acted as a predetermined breaking point. Therefore, the observed tensile curve of sample II cannot be safely verified by the other samples. At $10 \mathrm{wt} \%$ particle content, however, all samples display the described stress-strain behaviour (see Figure 4-13c). As all samples could be measured at similar ambient temperature, the obtained stress-strain curves and mechanical properties (see Table 4-8) reflect the accuracy of the tensile testing method. Similar to the aforementioned samples, the poly-APA grafted SNPs also caused strain whitening of the samples at all contents.

The observed change in the stress-strain behaviour, that is, a very linear increase of stress before sample failure, was also observed for $10 \mathrm{wt} \%$ of poly-MA grafted nanoparticles and unfunctionalized SNPs. At lower particle contents, the stress-strain curves resemble the behaviour of thermoplastics, that is, after the elastic region the stress remains almost constant which is due to cold-drawing. The chains adopt more stretched conformations and because the sample diameter decreases simultaneously, no rise in stress is observed. When all chains are completely stretched, a steep incease in stress occurs which is the observed strain-hardening before sample failure. Since the aforementioned nanocomposites show a different stress-strain behaviour, the deformation mechanism also needs to change. It may be suggested, that the presence of additional crosslinks superimposes the stretching of polymer chains. Therefore, while polymer chains are stretched, crosslinks are stressed simultaneously and eventually break which causes the stress to increase overall. Hence, the presented results 
suggest an influence of the SNPs at higher contents on the deformation mechanism which appears to be beneficial with regard to the mechanical properties of nanocomposites carrying $10 \mathrm{wt} \%$ of polymer-grafted SNPs.

Table 4-8 Mechanical properties of nanocomposites of the matrix copolymer APA$\mathrm{MA}^{\mathrm{MB}}$ and three different weight contents of poly-APA grafted silica nanoparticles. The respective stress-strain curves are depicted in Figure 4-13.

\begin{tabular}{lccc}
\hline \hline \multicolumn{1}{c}{ sample } & $\varepsilon_{\text {br }} / \%$ & $\sigma_{\text {UTS }} / \mathrm{MPa}$ & $U_{\mathrm{T}} / \mathrm{MPa}$ \\
\hline $1 \mathrm{wt} \%$ & & & \\
I $\left(24.0^{\circ} \mathrm{C}\right)$ & 804 & 0.4 & 2.2 \\
II $\left(24.0^{\circ} \mathrm{C}\right)$ & 719 & 2.9 & 8.8 \\
III $\left(23.3^{\circ} \mathrm{C}\right)$ & 735 & 2.1 & 8.3 \\
\hline $5 \mathrm{wt} \%$ & & & \\
I $\left(24.0^{\circ} \mathrm{C}\right)$ & 191 & 1.1 & 1.5 \\
II $\left(23.9^{\circ} \mathrm{C}\right)$ & 637 & 3.8 & 13.5 \\
III $\left(23.9^{\circ} \mathrm{C}\right)$ & 449 & 1.9 & 5.1 \\
\hline $10 \mathrm{wt} \%$ & & & \\
I $\left(24.0^{\circ} \mathrm{C}\right)$ & 692 & 5.7 & 17.0 \\
II $\left(24.0^{\circ} \mathrm{C}\right)$ & 647 & 5.0 & 17.7 \\
III $\left(24.0^{\circ} \mathrm{C}\right)$ & 722 & 3.1 & 13.7 \\
\hline \hline
\end{tabular}

Table 4-9 Glass transition temperatures that were determined for the nanocomposites of matrix copolymer APA-MA ${ }^{\mathrm{MB}}$ and three different weight contents of poly-APA grafted SNPs by means of DSC. Samples were chosen to aid in the interpretation of the tensile testing results.

\begin{tabular}{lc}
\hline \multicolumn{1}{c}{ sample } & $T_{\mathrm{g}} /{ }^{\circ} \mathrm{C}$ \\
\hline $1 \mathrm{wt} \%$ & \\
I $\left(24.0^{\circ} \mathrm{C}\right)$ & 13 \\
II $\left(24.0^{\circ} \mathrm{C}\right)$ & 19 \\
\hline $5 \mathrm{wt} \%$ & \\
II $\left(23.9^{\circ} \mathrm{C}\right)$ & 14 \\
\hline $10 \mathrm{wt} \%$ & \\
II $\left(24.0^{\circ} \mathrm{C}\right)$ & 17 \\
\hline \hline
\end{tabular}



crosslinking

\subsubsection{Conclusions}

In this chapter, the synthesis of silica nanoparticles and their subsequent functionalization with polymers has been described. Afterwards, unfunctionalized nanoparticles and polymer-grafted nanoparticles were used for the preparation of nanocomposite materials with three different weight contents of nanoparticles, by mixing with a biomimetic multiblock copolymer. For the preparation of polymergrafted nanoparticles, the unfunctionalized particles were immobilized with RAFT agent beforehand and then used in a polymerization with sacrificial RAFT agent in solution. Using TGA and DLS, the successful immobilization of RAFT agent and grafting of polymer could be verified. Nanocomposite materials were prepared by drop-casting of solutions that contained matrix copolymer and 1, 5 or $10 \mathrm{wt} \%$ of silica nanoparticles that were unfunctionalized or polymer-grafted, respectively.

The pristine matrix copolymer and the corresponding nanocomposites were then investigated by means of tensile testing. It could be demonstrated that addition of $1 \mathrm{wt} \%$ of SNPs, functionalized or unfunctionalized, improved mechanical properties such as tensile strength and toughness. For one sample (sample III, $1 \mathrm{wt} \%$, poly-MA functionalized SNPs) an almost 8-fold increase of sample toughness was noted. Additionally, the stress-strain behaviour of the samples varied with increasing particle content. Strain-hardening was reduced and a more linear increase of stress over strain resulted. Despite finding these beneficial effects of preparing nanocomposites, it has to be noted that reproducibility was relatively poor for some particle contents due to inhomogeneities that resulted in void formation during the solventcasting process or necking during tensile testing. Also, the lack of temperature control impedes further interpretation of the obtained results, as the different ambient temperatures seemed to have influenced the stress-strain behaviour of several samples. Hence it would be desirable to investigate the nanocomposite materials via dynamic mechanical analysis, as the sample modulus is measured versus temperature and the mechanical properties of the nanocomposites could hence be evaluated more comprehensively. ${ }^{166,212,214}$

Furthermore, as the stress-strain curves suggested agglomeration and/or demixing of SNPs, independent of the particle surface, it would be worthwhile to investigate the dispersion of the particles. Scanning electron microscopy ${ }^{141,215,216}$ (SEM) or transmission electron microscopy183,217,218 (TEM) could be used to determine the dispersion of particles on the surface of a bulk sample (SEM) or of a thin film (TEM). 
Atomic force microscopy (AFM) may also be used to investigate surface morphologies of thin films. ${ }^{184}$ Concerning the particle dispersion it might also be attempted to change the processing conditions. Melt mixing of the respective components may be used or other solvents than PGMEA could be utilized to influence particle dispersion. ${ }^{219}$ One sample using unfunctionalized SNPs at $10 \mathrm{wt} \%$ content also showed a different stress-strain behaviour after storing it for $36 \mathrm{~h}$ at room temperature. It was suggested that the particles could aggregate and were expelled from the polymer matrix. Thus it would be worthwhile to also investigate the time dependence of especially polymer-grafted SNPs further as these should improve interaction between SNPs and matrix copolymer.

A very sophisticated approach towards improvement of the performance of silica nanocomposites is the synthesis of bimodal silica nanoparticles. ${ }^{183,195}$ Using this approach, the dispersion and the formation of superstructures of nanoparticles in a polymer matrix may be controlled.175,180,183 As the here presented multiblock copolymers are in the rubbery state, that is, above the glass transition temperature of one component, one would aim to realize percolation networks of the particles to improve the mechanical properties of nanocomposites even further. ${ }^{179,186}$ Nevertheless, the here presented approach was successful in improving the mechanical properties of the biomimetic copolymers significantly.

\subsection{Biomimetic star block copolymers via RAFT polymerization using the Z-group approach ${ }^{3}$}

As already explained in the preface of this chapter (see section 4.1), switching the macromoleular architecture from linear polymer chains to a star topology was selected as the second approach to obtain biomimetic copolymers. Especially with the advent of RDRP techniques such as RAFT or atom transfer radical polymerization (ATRP), a plethora of techniques became available that may be utilized to form star-like macromolecular structures. ${ }^{38}$ This promoted extensive research, leading to a comprehensive understanding of the special properties of star polymers. Compared to linear polymers of the same molar mass, star polymers exhibit a smaller hydrodynamic volume which consequently

\footnotetext{
${ }^{3}$ The results that are reported in this section are part of the bachelor thesis „Synthese von Sternpolymeren mit wasserstoffbrückenbindenden Segmenten via RAFT-Polymerisation und Untersuchung der mechanischen Eigenschaften“ by Darius Rohleder.
} 

crosslinking

leads to lower viscosity of starpolymers in solution and in melt. This renders them ideal for industrial applications as viscosity modifiers in, for example, adhesives and coatings. ${ }^{38}$ Especially as additives in engine oils, star polymers gained importance because they provide better low temperature fluidity and hence lead to better fuel efficiency and economy. By the design of amphiphilic star block copolymers, the special macromolecular architecture results in the formation of core-shell structures in solution. ${ }^{37,38,220}$ These star block copolymers may be utilized in various ways, such as tailored formation of nanostructures in solution $^{220}$ or as single molecule drug carriers. ${ }^{38}$ The latter can also be approached in a very elegant fashion by the synthesis of hollow-sphere particles using 6-arm Z-star RAFT agents as starting molecules. ${ }^{37}$ In these star RAFT agents, the RAFT groups are always attached to the star core. After the preparation of amphiphilic diblock copolymers, the outer layer of the star molecules is crosslinked via ultra violet (UV)-irradiation and subsequent aminolysis removes the RAFT groups, leaving hollow particles that may be loaded with drug molecules.

Though the aformentioned examples exclusively focused on the application of star polymers due to their solution properties, they may also contribute beneficially to the mechanical properties of bulk polymer materials. When linear triblock and star shaped diblock copolymers diplaying similar block lengths are compared by tensile testing, an increase of the ultimate strength ${ }^{85,172}$ and toughness ${ }^{173}$ are observed. A toughening of the polymeric material may occur as the result of change in the microphase morphology when the block copolymers show phase separation, which impacts the deformation mechanism. ${ }^{173}$ In star diblock copolymers that comprise soft and hard polymer segments, the hard segments form smaller domains than in linear triblock copolymers. ${ }^{221} \mathrm{~A}$ larger number of hard domains per unit volume is present and the effective surface of these domains is elevated, ultimately leading to an increased influence on the mechanical properties. ${ }^{85,172}$ This effect is similar to composite materials that comprise nanoscopic instead of microscopic filler materials (also see section 4.2). ${ }^{189,190}$ Additionally, stresses are more evenly distributed to the hard domains due to uniform arm length. Therefore, star block copolymers are less suspectible to stress concentration which eventually leads to catastrophic material failure.

Besides the higher resilience of star diblock copolymers to mechanical stresses compared to linear triblock copolymers, the star copolymers also exhibit a lower viscosity in the melt state. This is crucial 
for industrial applications as the processability of the polymers is improved. ${ }^{85}$ With increasing number of arms, the star copolymers also become less susceptible to the processing conditions with regard to the resulting mechanical performance whereas the mechanical properties of linear triblock copolymers highly depend on the processing conditions. For instance, properties such as tensile strength are superior when linear polymers are processed via solvent casting instead of molding, as the molding process hampers microphase separation. ${ }^{85}$ In star block copolymers, however, microphase separation is facilitated and the respective microphase morphology is developed under either conditions. ${ }^{85,222}$ As a result, star block copolymers also maintain higher levels of tensile strength at elevated temperatures compared to their linear counterparts. ${ }^{85,172}$

The previous remarks demonstrate that it is worthwhile to design star block copolymer analogs of the presented biomimetic linear block copolymers (see chapter 3 ) in order to obtain materials with better mechanical performance. In the following, the synthesis of star diblock copolymers is described that, in accordance with the biomimetic approach, also comprise poly(methyl acrylate) to form soft, elastic segments and poly-APA to form stiff and strong segments. Afterwards, characterization of the prepared star polymers via tensile testing and DSC is presented.

\subsubsection{Synthesis}

For the synthetic implementation of star polymers, two techniques can be distinguished: the arm-first and the core-first approach. While the first one follows an initial synthesis of the arms that are afterwards connected via a crosslinker, the second approach starts from a precursor molecule that constitutes the star shape. The latter can be devided even further into the R- and Z-group approach. In the R-group approach, reactive sites are attached to the star core whereas in the Z-group approach the reactive species are attached to the growing arms. Both, arm-first technique and the core-first R-group approach may be realized by a multitude of polymerization techniques such as anionic and ringopening polymerizatins or the most prominent RDRP techniques, that is, RAFT, ATRP and nitroxide-mediated radical polymerization (NMP). ${ }^{38}$ The Z-group approach, however, is unique to the RAFT process since only RAFT agents may be designed such that the radical functionality is not attached to the star core during polymerization.223-226 Star-star coupling events are hence significantly suppressed by the Z-group 

crosslinking

approach which is usually regarded as the major advantage of this method. ${ }^{225}$ However, it has to be noted that star-star coupling reactions can occur in polymerizations of, for instance, $n$-butyl acrylate at high conversions. ${ }^{227}$ Then, intermolecular chain transfer may result in armcentered radical species that ultimately lead to the coupling of star species. As this should not be a concern in the present study, a Z-group star RAFT agent was chosen to prepare star diblock copolymers. Also, it should be emphasized that the R-group approach is in principle also capable of yielding very uniform star polymers when the polymerization conditions are carefully chosen. ${ }^{224,225}$

The utilized RAFT agent is depicted in Figure 4-14. It was used by Skey et al. ${ }^{220}$ for the design of amphiphilic star diblock copolymers that underwent self-assembly in solution, using a derivatized APA monomer carrying a carboxy methyl ester functionality. Therefore, it could be reasoned that the star RAFT agent would also be capable of controlling the polymerization of APA monomer. The order of monomer addition in the polymerization process is analogous to the presented linear block copolymers, that is, APA needs to be polymerized first so that the polyAPA segments would form the end segments of the arms. The mechanism of monomer addition to a Z-RAFT star is schematically depicted in Scheme 4-3.

\subsubsection{Synthesis of poly-APA star homopolymers}

Though the work of Skey et al.220 had applied the methyl ester derivative of APA, no work could be found in literature that utilized APA carrying an unprotected carboxylic acid group in polymerizations with the respective star RAFT agent (see Figure 4-14). Therefore, polymerization series were conducted to investigate the quality of star-RAFT-1 as RAFT agent for the polymerization of APA. The results including SEC analysis of the obtained polymers are collated in Table 4-10.

It has to be noted, that for sample $\sigma$-i a higher monomer concentration was applied than for the samples $\sigma$-ii to $\sigma$-v. Therefore, the obtaind results are discussed separately. Evaluation of the SEC data reveals that despite increasing consumption of monomer with time no simultaneous increase in number average molar mass $\bar{M}_{\mathrm{n}, \mathrm{SEC}}$ can be observed. For comparison, the theoretical number average molar masses $\bar{M}_{\mathrm{n} \text {,Theory }}$ that can be calculated based on the conversions are also presented. Along with dispersity values of around 1.5 these results suggest a mediocre control over the polymerization of APA by the applied star RAFT agent. 
<smiles>O=C(CCSC(=S)SCc1ccccc1)OCC(COC(=O)CCSC(=S)SCc1ccccc1)(COC(=O)CCSC(=S)SCc1ccccc1)COC(=O)CCSC(=S)SCc1ccccc1</smiles>

star-RAFT-1

Figure 4-14 Star RAFT agent star-RAFT-1 that was used for the design of biomimetic star diblock copolymers.

These results, however, agree well with the results obtained for the linear poly-APA (see section 3.2.2) and they also agree well with results that were reported by Skey et al. ${ }^{220}$ They used even higher monomer concentrations than applied here and varied temperature between 60 and $90^{\circ} \mathrm{C}$, reporting dispersities between 1.4 and 1.7. Additionally, the relatively low apparent molar masses $\bar{M}_{\mathrm{n}, \mathrm{SEC}}$ suggest a certain level of control because Mori et al.119 conducted conventional radical polymerization of APA under similar conditions and obtained number average molar mass of about $60000 \mathrm{~g} \mathrm{~mol}^{-1}$ versus polystyrene standards.

As already outlined in section 3.2.2, APA is a highly substituted monomer which is prone to transfer reactions, thereby contributing to a non-uniformity of chains and an overall reduction of control. A different issue might be poor initiation of arm growth. To obtain uniform arm lenghts a simultaneous initiation of all star arms is crucial. ${ }^{130}$ Inadequate choice of RAFT agent with respect to the reinitiating $\mathrm{R}$ group may only lead to fully initiated star polymers at high monomer conversions which consequently results in star polymers that lack topological control. Additionally, the methylation process (see section 3.2 .2 and experimental section 7.3.8.4) that is required post-polymerization to prepare APA-polymers for SEC might have not worked quantitatively, leaving carboxylic acid groups unprotected which could adhere to the 

crosslinking

SEC column material and falsify the obtained molar mass distributions. Besides, as SEC is predominantly measured versus polystyrene standards in this work, obtained results have to be carefully interpreted as they only give insight into the corresponding hydrodynamic radius of the star polymers but not their actual molar mass. Termination events naturally contribute to broadening of the molar mass distributions, especially for longer reaction times (samples $\sigma$-iv and $\sigma$-v) as dead arms increasingly contribute to comparably low molar mass species.

After evaluating the development of molar mass distributions with time, sample $\sigma$-i needs to be discussed, as a higher monomer concentration was used for this sample. The sample shows almost quantitative monomer conversion (see Table 4-10) after $2 \mathrm{~h}$, whereas the lower monomer concentration gave quantitative conversion after more than $24 \mathrm{~h}$. Additionally, a significantly higher apparent $\bar{M}_{\mathrm{n}, \mathrm{SEC}}$ is determined with comparable dispersity. Both observations are expected, as a higher monomer concentration leads to a higher monomer to RAFT ratio and faster polymerization rate. ${ }^{64}$ It can be argued that reducing the reaction time also leads to fewer termination events and dead chains, as fewer initiator derived chains are generated in the polymerization process. This could also lead to a narrowing of the molar mass distribution. Following this reasoning, an additional polymerization series was conducted with the monomer concentration of sample $\sigma$-i but at $90{ }^{\circ} \mathrm{C}$. To assure similar decay kinetics to AIBN at $60^{\circ} \mathrm{C}$ and hence a comparable number of radical species generated in a similar timeframe, 1,1'azobis(cyclohexanecarbonitrile) (ABCN) was used as initiator as it possesses a similar half life period at $90^{\circ} \mathrm{C}$.

In these experiments, quantitative monomer conversion was already obtained after $5 \mathrm{~min}$. However, dispersities were initially larger than 2, showing a significant loss of control which therefore rendered the reaction conditions inept for further work. Side reactions such as transfer to monomer probably become more dominant at elevated temperatures and override possible benefits of fewer termination events. As the experiments were unsuccessful, the results are not shown here in further detail. To synthesize sufficient amounts of material for investigations via tensile testing an upscaling of the reaction was necessary as the polymerization series were conducted in $5 \mathrm{~mL}$ vials in a thermostated heating block. Conducting the polymerization reaction in a $250 \mathrm{~mL}$ flask in an oilbath for $8 \mathrm{~h}$ only yielded a monomer conversion of about $20 \%$ and also significantly higher dispersities. This is most likely caused by a more inhomogeneous temperature distribution of the larger 
reaction volume. After additional attempts, a large amount of poly-APA star homopolymer (sample $\sigma$-vi, see Table 4-10) was obtained after a reaction time of $10 \mathrm{~h}$. Results of the SEC characterization yielded an apparent $\bar{M}_{\mathrm{n}, \mathrm{SEC}}=8400 \mathrm{~g} \mathrm{~mol}^{-1}$ and dispersity $\oslash=1.7$. The conversion was determined to be $43 \%$. Concerning the target degree of polymerization of 160 APA monomer units per star RAFT, this corresponds to approximately 68 APA monomer units per star or, on average, 17 monomer units per arm.

To verify a uniform arm length of the prepared star homopolymer, the trithiocarbonate RAFT groups may be cleaved via aminolysis. Here, a method according to Shen et al.228 was followed using hydrazine as cleaving agent. The obtained SE chromatograms of the star polymer before and after aminolysis are shown in Figure 4-15. The peak molar mass $M_{\mathrm{P}}$ shifts from $3.810^{4} \mathrm{~g} \mathrm{~mol}^{-1}$ for the original star polymer to $1.410^{4} \mathrm{~g} \mathrm{~mol}^{-1}$ for the cleaved chains which corresponds to a factor of 2.7. Additionally, the dispersity decreases from 1.7 to 1.5 . Ideally, the difference in molar mass would be expected to be a factor of four. It has to be taken into account that star polymers exhibit a smaller hydrodynamic volume compared to linear polymers of the same molar mass, which would reduce the factor of four. ${ }^{226}$ Concerning the dispersity of the initial and cleaved polymer, however, an estimation of the uniformity of the arms is impeded as the obtained molar mass distributions are too broad. The cleavage experiments hence do not yield qualitative information on the uniformity of arms. In summary, the controlled synthesis of poly-APA star homopolymers proved to be a challenging task which is presumably hampered due to the highly substituted monomer APA. Nonetheless, the star homopolymer could be produced on a gram-scale with a medium level of control over the polymerization. The results of the corresponding copolymerizations with MA will be discussed in the following section. 
Table 4-10 Results of the polymerization series of APA in 1,4-dioxane at $60^{\circ} \mathrm{C}$. SEC was measured versus linear polystyrene standards. Conversions were determined gravimetically. For sample $\sigma$-i a 1.65 -fold higher monomer concentration was used compared to the other samples. The target degree of polymerization was 160 APA monomer units per star RAFT molecule. Sample $\sigma$-vi was obtained after upscaling of the reaction and was consequently used as macro-RAFT agent for copolymerizations.

\begin{tabular}{cccccc}
\hline sample & $\begin{array}{c}\text { time } \\
\mathrm{h}\end{array}$ & $\begin{array}{c}\text { conversion } \\
/ \%\end{array}$ & $\begin{array}{c}\bar{M}_{\mathrm{n}, \mathrm{SEC}} / \\
10^{4} \mathrm{~g} \mathrm{~mol}^{-1}\end{array}$ & $\begin{array}{c}\bar{M}_{\mathrm{n}, \text { Theory }} / \\
10^{4} \mathrm{~g} \mathrm{~mol}^{-1}\end{array}$ & Ð \\
\hline$\sigma$-i & 2 & 98 & 1.8 & 6.2 & 1.5 \\
$\sigma$-ii & 4 & 39 & 0.9 & 1.6 & 1.5 \\
$\sigma$-iii & 8 & 69 & 1.2 & 2.7 & 1.4 \\
$\sigma$-iv & 24 & 93 & 0.6 & 3.6 & 1.7 \\
$\sigma-\mathrm{v}$ & 48 & 100 & 1.1 & 3.8 & 1.6 \\
$\sigma$-vi & 10 & 43 & 0.8 & 1.6 & 1.7 \\
\hline \hline
\end{tabular}

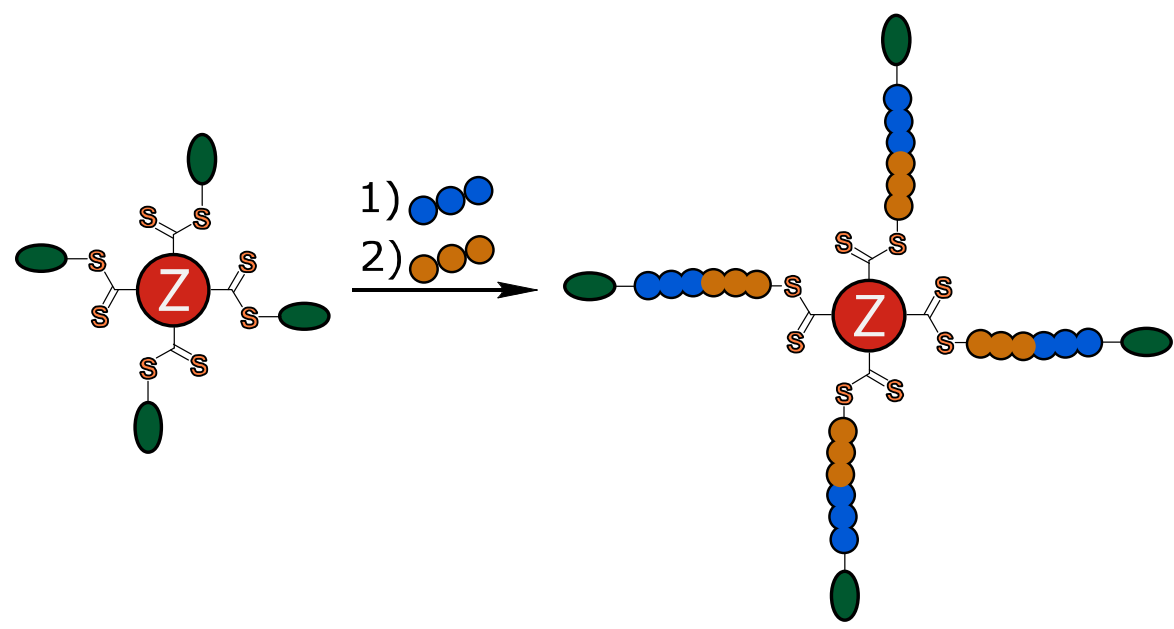

Scheme 4-3 Schematic illustration of the formation of star diblock copolymers via the sequential addition of two monomers (blue and orange circles) using the Z-group (red circle) approach. In this work, the first monomer is the hydrogen bonding APA and the second monomer is MA. It is important to note that the RAFT groups are always attached to the star core. The R-group is depicted as a green ellipse 


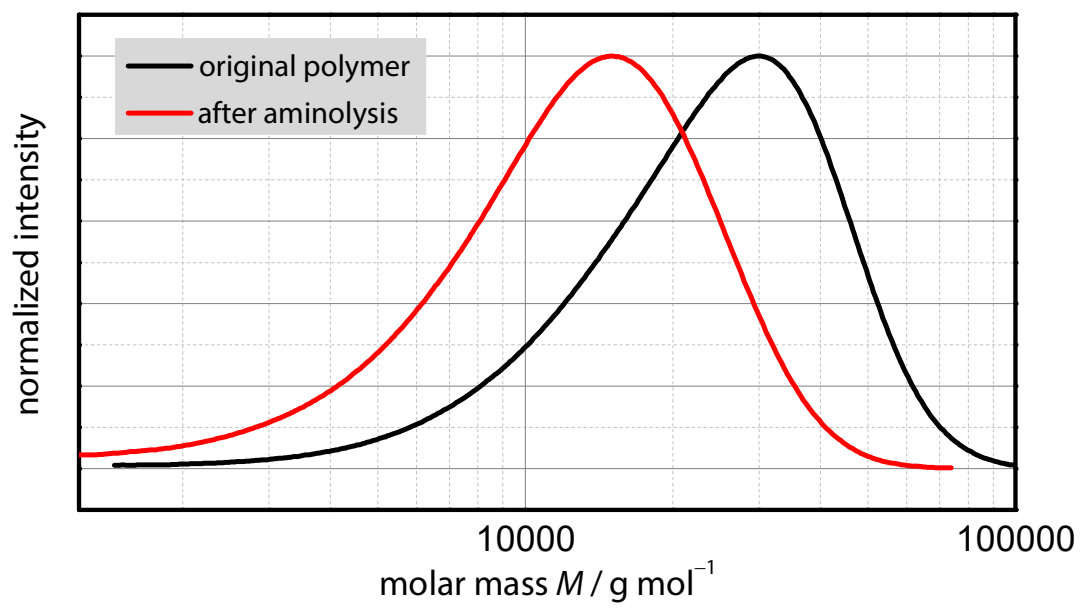

Figure 4-15 SE chromatograms (DMAc) of the poly-APA star homopolymer $\sigma$-vi before and after aminolysis as recorded by RI detection.

\subsubsection{Synthesis of poly(APA-block-methyl acrylate) star diblock copolymers}

In accordance with the preliminary work performed for the linear block copolymers (see section 3.2.1), copolymerizations of the poly-APA star polymers with MA had to be conducted under the premise of obtaining materials with a sufficient dimensional stability to prepare specimens for tensile testing. Since no literature existed on copolymerizations involving the prepared star polymers, a polymerization series was conducted. The star homopolymers were utilized as macromolecular RAFT (macro-RAFT) agents. To determine a reasonable target degree of polymerization for MA, the APA content of the star homopolymers needs to be considered. As explained in the previous section, the star polymers should display on average 17 APA monomer units per arm. This content appears to be well comparable to the linear block copolymers that contained on average 10 APA monomer units per RAFT group. In preliminary work it was determined that a monomer ratio of $10 \mathrm{APA}$ to $150 \mathrm{MA}$ monomer units renders the linear block copolymers too brittle to be usable in tensile testing (see section 3.2.1). For the desired 4-arm star copolymers this would correspond to a target degree of polymerization of at least 600 for MA. The upper limit of MA 

crosslinking

content were 300 monomer units per 10 units of APA. Therefore, it was decided to apply a target degree of 1400 MA units per macro-RAFT molecule to assure that a sufficient amount of MA would be incorporated into the star copolymers.

Samples were run for different reaction times and the conversions of MA were determined gravimetically. All copolymers were characterized by means of SEC and the results are collated in Table 4-11 (SE chromatograms are shown in Figure 4-16). The SEC characterization yielded several remarkable results. Very high molar mass species are observable that are especially dominant in the samples $\sigma$-I and $\sigma$-III, resulting in particularly high dispersities. This observation may indicate aggregation of star copolymers which would be promoted by incomplete methylation of the carboxylic acid groups in APA. DLS was performed as an independent method.

To investigate the presumed aggregation of star polymers, copolymer sample $\sigma$-I was dissolved in $\mathrm{N}, \mathrm{N}$-dimethylacetamide (DMAc), that is, the SEC eluent and measured at $35^{\circ} \mathrm{C}$ to resemble the experimental conditions. The star homopolymer $\sigma$-vii was measured for comparison. Both intensity distributions are depicted in Figure 4-17. Both, homopolymer and copolymer display two distinct maxima, with a relatively sharp distribution at small hydrodynamic radius and a broader distribution at large hydrodynamic radius. It appears reasonable that the former corresponds to unimolecular species and the latter to molecular aggregates. Therefore, the results of the DLS measurements give evidence for an aggregation occuring during the SEC analysis. This precludes an evaluation of the degree of control of the copolymerizations. Furthermore, the reproducibility of the material synthesis can be assumed to be rather poor.

Nonetheless it was decided to investigate the samples $\sigma$-III, $\sigma$-IV and $\sigma$-VI via tensile testing as these samples exhibited a tough texture and a basic influence of the macromolecular architecture on the mechanical properties could be investigated. Additionally, DSC experiments were performed on the aforementioned copolymers and homopolymers that are listed in Table 4-10 to examine an influence on thermal transitions of the polymer. 
Table 4-11 Results of the copolymerization series that used the macro-RAFT agent $\sigma$ vi (see Table 4-10) in methanol at $60^{\circ} \mathrm{C}$. SEC was measured versus linear polystyrene standards. Conversions were determined gravimetically. The respective SEC curves are shown in Figure 4-16.

\begin{tabular}{|c|c|c|c|c|c|}
\hline sample & $\begin{array}{c}\text { time / } \\
\mathrm{h}\end{array}$ & $\begin{array}{c}\text { conversion } \\
/ \%\end{array}$ & $\begin{array}{c}\bar{M}_{\mathrm{n}, \mathrm{SEC}} / \\
10^{4} \mathrm{~g} \mathrm{~mol}^{-1}\end{array}$ & $\begin{array}{c}\bar{M}_{\mathrm{n}, \text { Theory }} / \\
10^{4} \mathrm{~g} \mathrm{~mol}^{-1}\end{array}$ & 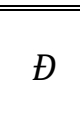 \\
\hline$\sigma-\mathrm{I}$ & 0.25 & 35 & 0.8 & 5.3 & 668 \\
\hline$\sigma$-II & 0.5 & 41 & 2.8 & 6.0 & 7.82 \\
\hline$\sigma$-III & 1 & 49 & 2.5 & 7.0 & 134 \\
\hline$\sigma$-IV & 2 & 67 & 3.6 & 9.2 & 2.86 \\
\hline$\sigma-\mathrm{V}$ & 10 & 80 & 2.4 & 10.7 & 3.57 \\
\hline$\sigma-\mathrm{VI}$ & 12 & 85 & 3.9 & 11.3 & 6.94 \\
\hline
\end{tabular}

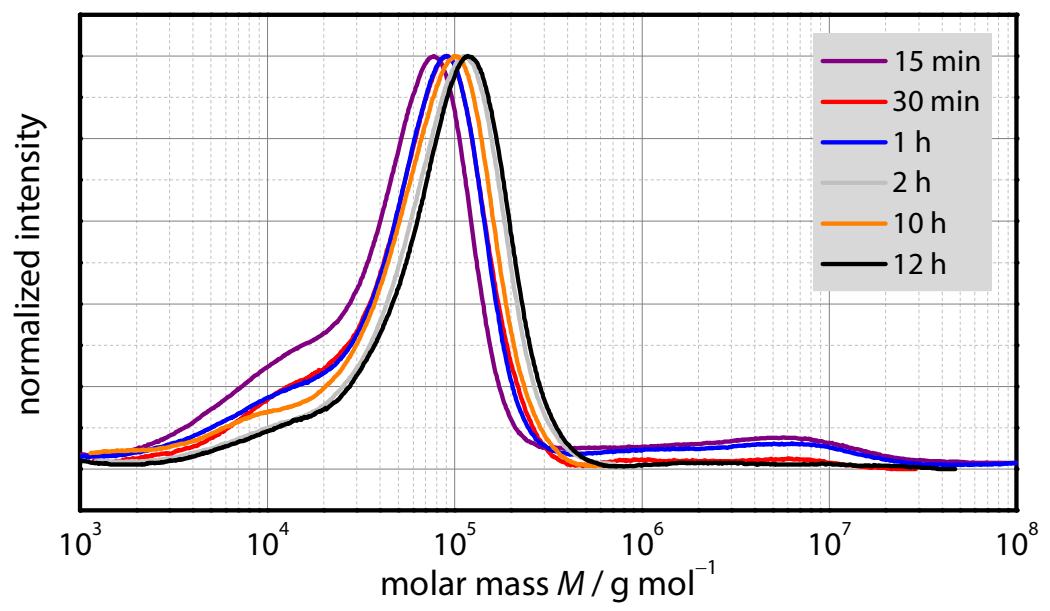

Figure 4-16 SE chromatograms (DMAc) of the star diblock copolymer samples (see Table 4-11) as recorded by RI detection. 


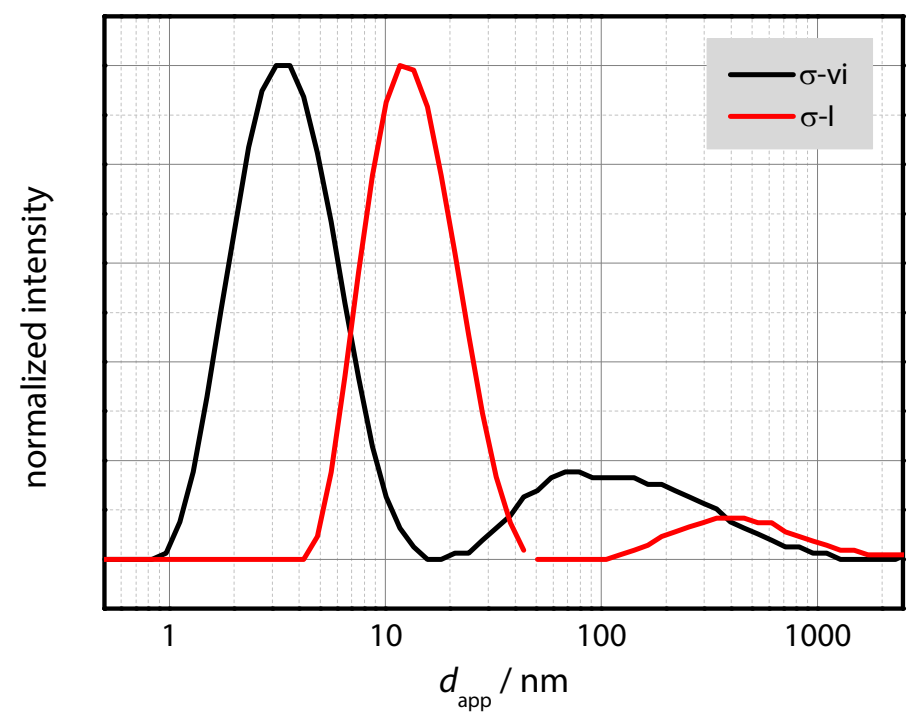

Figure 4-17 Normalized intensity distributions that were obtained via DLS for the polyAPA star homopolymer $\sigma$-vii (see text) and the copolymer $\sigma$-I (see Table 4-11).

\subsubsection{Differential scanning calorimetry}

First, the prepared star homopolymers (see Table 4-10) were investigated via DSC, the corresponding glass transition tempratures are presented in Table 4-12. For the linear poly-APA (see section 3.2.2) samples, glass transition temperatures of 115 and $127^{\circ} \mathrm{C}$ could be determined for single and multiblock poly-APA. The star polymers, however, display significantly higher glass transition temperatures, ranging from 145 to $157^{\circ} \mathrm{C}$. Sample $\sigma$-i exhibits the highest glass transition temperature which is expected as the glass transition temperature of a polymer increases with increasing molar mass to a material dependent threshold value. ${ }^{55}$ Despite having a medium molar mass with respect to the other samples, polymer $\sigma$-v shows the lowest glass transition temperature. This is presumably due to a larger amount of terminated low molar mass chains which act as plasticizers and hence decrease the glass transition temperature. Since all samples display an even higher glass transition temperature than their linear counterparts, poly-APA domains should also be capable of providing sufficient strength to the corresponding star copolymers. 
Table 4-12 Glass transition temperatures of the homopolymer samples that are presented in Table 4-10.

\begin{tabular}{ccc}
\hline \hline sample & $\begin{array}{c}\bar{M}_{\mathrm{n}, \mathrm{SEC}} / \\
1 \mathrm{~g} \mathrm{~mol}^{-1}\end{array}$ & $T_{\mathrm{g}} /{ }^{\circ} \mathrm{C}$ \\
\hline$\sigma$-i & 1.8 & 157 \\
$\sigma$-ii & 0.9 & 153 \\
$\sigma$-iii & 1.2 & 155 \\
$\sigma$-iv & 0.6 & 151 \\
$\sigma$-v & 1.1 & 145 \\
\hline \hline
\end{tabular}

The DSC curves of the copolymer samples that were subsequently investigated via tensile testing are depicted in Figure 4-18. Glass transition temperatures are collated in Table 4-13. All copolymer samples exhibit one pronounced glass transition at about $17^{\circ} \mathrm{C}$ which corresponds to segments of poly-MA. Compared to pure poly-MA $\left(T_{\mathrm{g}}=9^{\circ} \mathrm{C}\right.$, see section 3.2.2) an elevated glass transition temperature is determined, which proves an effective confinement that is imposed onto the poly-MA segments by poly-APA domains. However, no profound impact of the molar mass of the poly-MA segments on the glass transition temperature can be determined, which agress well with the results of the linear copolymers. Furthermore, glass transitions of the poly-APA segments are absent in the copolymers. This indicates lack of phase separation in the copolymers which may be expected due to the high dispersities of the copolymers (see Table 4-11). In total, the DSC measurements demonstrate an effect of the poly-APA segments on the glass transition temperature of the poly-MA domains which indicates formation of physical crosslinks by the poly-APA segments. These should contribute beneficially to the mechanical properties which will be presented in the following section. 
4 Tuning the mechanical properties of biomimetic copolymers via crosslinking

Table 4-13 Glass transition temperatures of copolymer samples $\sigma$-III, $\sigma$-IV and $\sigma$-VI (see Table 4-11) that were obtained from the respective DSC curves (see Figure 4-18).

\begin{tabular}{ccc}
\hline \hline sample & $\begin{array}{c}\bar{M}_{\mathrm{n}, \mathrm{SEC}} / \\
10^{4} \mathrm{~g} \mathrm{~mol}^{-1}\end{array}$ & $T_{\mathrm{g}} /{ }^{\circ} \mathrm{C}$ \\
\hline$\sigma$-III & 2.5 & 18 \\
$\sigma$-IV & 3.6 & 16 \\
$\sigma$-VI & 3.9 & 17 \\
\hline \hline
\end{tabular}

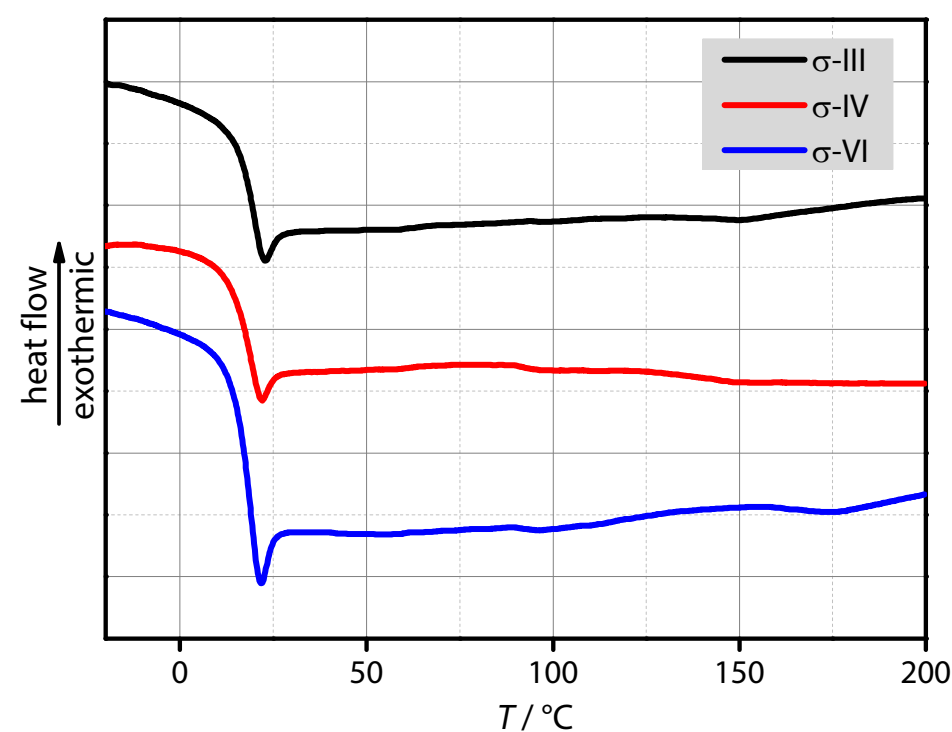

Figure 4-18 DSC curves of copolymer samples $\sigma$-III, $\sigma$-IV and $\sigma$-VI that are presented in Table 4-11. Curves are shifted vertically for clarity. Glass transition temperatures that were obtained from these curves are collated in Table 4-13.

\subsubsection{Tensile testing}

Similar to the linear biomimetic copolymers, dogbone specimens of the star copolymers were prepared via drop-casting. PGMEA was used as solvent as it was also capable of dissolving the star copolymers. Due to its high boiling point $\left(T_{\mathrm{b}}=146^{\circ} \mathrm{C}\right)$, it should also reduce the formation of bubbles in the specimens during the casting process. However, the preparation of applicable specimens emerged to be a highly challenging 
task for the star copolymers. During the process of solvent removal under vacuum, extensive formation of bubbles was observed especially for sample $\sigma$-III (compare Figure 3-8) that leads to the formation of voids. This can be most likely explained by the lower poly-MA content which increases the density of physical crosslinks formed by the polyAPA segments, thus hindering the diffusion of solvent molecules. However, the solvent could not be exchanged as the copolymers were insoluble in other common solvents such as toluene. Finally, a reduction of the number of voids could be achieved by conducting the evaporation process very slowly, which required more than one week to prepare a specimen of $\sigma$-III that was applicable in tensile testing. However, the specimen still displayed minor voids which are likely to deteriorate the mechanical properties. Specimens of the samples $\sigma$-IV ( 5 days) and $\sigma$-VI (3 days) could be prepared in less than one week, and one specimen of each copolymer was then investigated via tensile testing. The obtained stress-strain curves are depicted in Figure 4-19 and the respective data are collected in Table 4-14.

As expected, the elongation at break increases with increasing content of poly-MA, that is, from $\sigma$-III to $\sigma$-VI due to the longer polymer chains. Sample $\sigma$-III shows a lower tensile strength than $\sigma$-IV which is expected to be the other way round due to the higher crosslink density, however, as the specimen exhibited minor voids this is the result of premature failure of the test specimen. Concerning these preliminary results, one might suggest that $\sigma$-VI exhibits the best comonomer composition of the three samples as it yielded the best mechanical performance with respect to tensile strength and resulting sample toughness. However, no additional test specimens were prepared as these preliminary results already demonstrate an inferior mechanical performance compared to the linear block copolymers. Additionally, it was attempted to regenerate the tensile specimen of sample $\sigma$-VI but unlike the linear block copolymers a healing process did not occur. These results, in addition to the poor processability of the samples, showed that the change of macromolecular architecture in the presented approach may not yield materials superior to the presented linear biomimetic copolymers (see chapter 3 ). 
4 Tuning the mechanical properties of biomimetic copolymers via crosslinking

Table 4-14 Overview of the mechanical properties of the star diblock copolymer samples $\sigma$-III, $\sigma$-IV and $\sigma$-VI. The quantities were obtained from a single stress-strain curve.

\begin{tabular}{ccccc}
\hline \hline sample & $E / \mathrm{MPa}$ & $\varepsilon_{\text {br }} / \%$ & $\sigma_{\text {UTS }} / \mathrm{MPa}$ & $U_{\mathrm{T}} / \mathrm{MPa}$ \\
\hline$\sigma$-III & 3.4 & 181 & 0.1 & 1.1 \\
$\sigma$-IV & 3.2 & 363 & 1.32 & 3.0 \\
$\sigma$-VI & 0.63 & 539 & 0.43 & 1.1 \\
\hline \hline
\end{tabular}

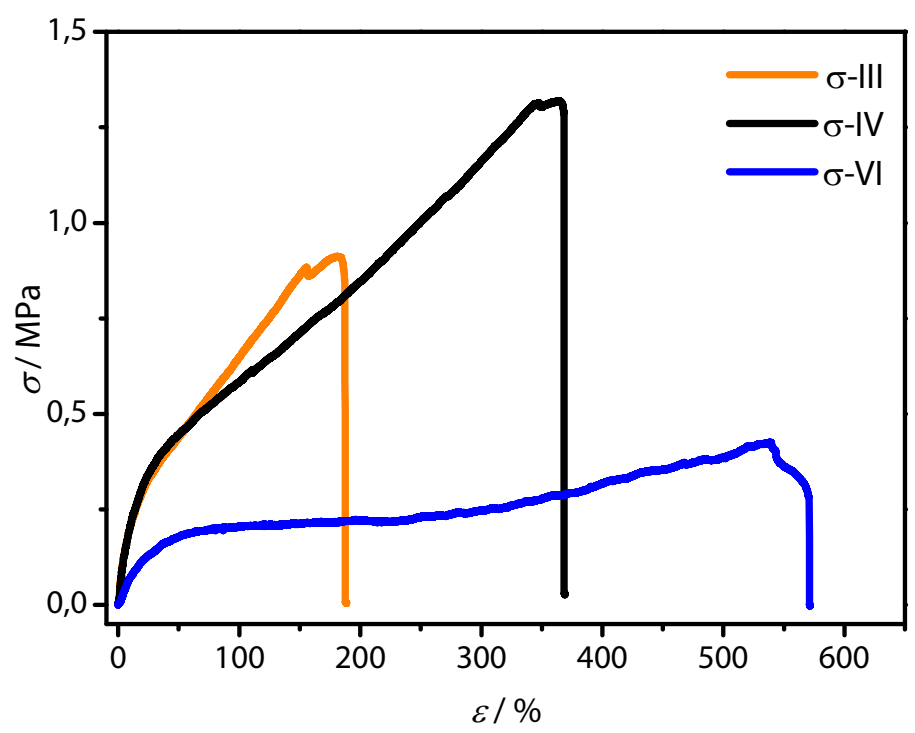

Figure 4-19 Stress-strain curves of the star diblock copolymers $\sigma$-III (red), $\sigma$-IV (black) and $\sigma$-VI (blue). Obtained characteristic quantities from these curves are collated in Table 4-14

\subsubsection{Conclusions}

In summary, the synthesis and characterization of the mechanical properties of star diblock copolymers were presented. Using this approach, it was attempted to surpass the mechanical properties of linear block copolymers that are based on the same comonomer combination. However, the synthesis proved to be unequally more challenging as, for instance, upscaling of the homopolymer synthesis was not straightforward and the characterization of the copolymers via SEC 
was hindered due to aggregate formation. Ultimately, the preparation of tensile specimens of the prepared copolymers via drop-casting could not be adequately conducted as one sample always displayed voids. Both material synthesis and the mechanical testing appear to be poorly reproducible due to the encountered issues, rendering the approach inferior to the biomimetic multiblock strategy. However, in the next chapter an improved approach involving star polymers is presented that is promising to surpass the mechanical properties of the biomimetic polymers that were presented in chapter 3 .

\subsection{Multiblock copolymer materials via mixing of polyfunctional and star RAFT agents ${ }^{4}$}

In the previous section it was discussed that star diblock copolymers with hydrogen bonding outer segments may cause difficulties with regard to processability and characterization of the polymer materials. Presumably, these issues occured because macromolecules may form relatively dense networks consisting of physical crosslinks formed by the hydrogen bonding segments and chemical crosslinks resembled by the star cores. In order to improve the processability one may rationale that the crosslink density should be decreased. The density of physical crosslinks formed by hydrogen bonding segments may be reduced by increasing the overall molar mass while keeping the hydrogen bonding block length constant. As higher molar mass facilitates the formation of entanglements, 55,139 that is, a different type of physical crosslink, this strategy should result in similar difficulties. However, reducing the density of chemical crosslinks can be realized with ease by blending star polymers with linear polymers.

${ }^{4}$ Hendrich, M. and Vana, P. manuscript in preparation. 


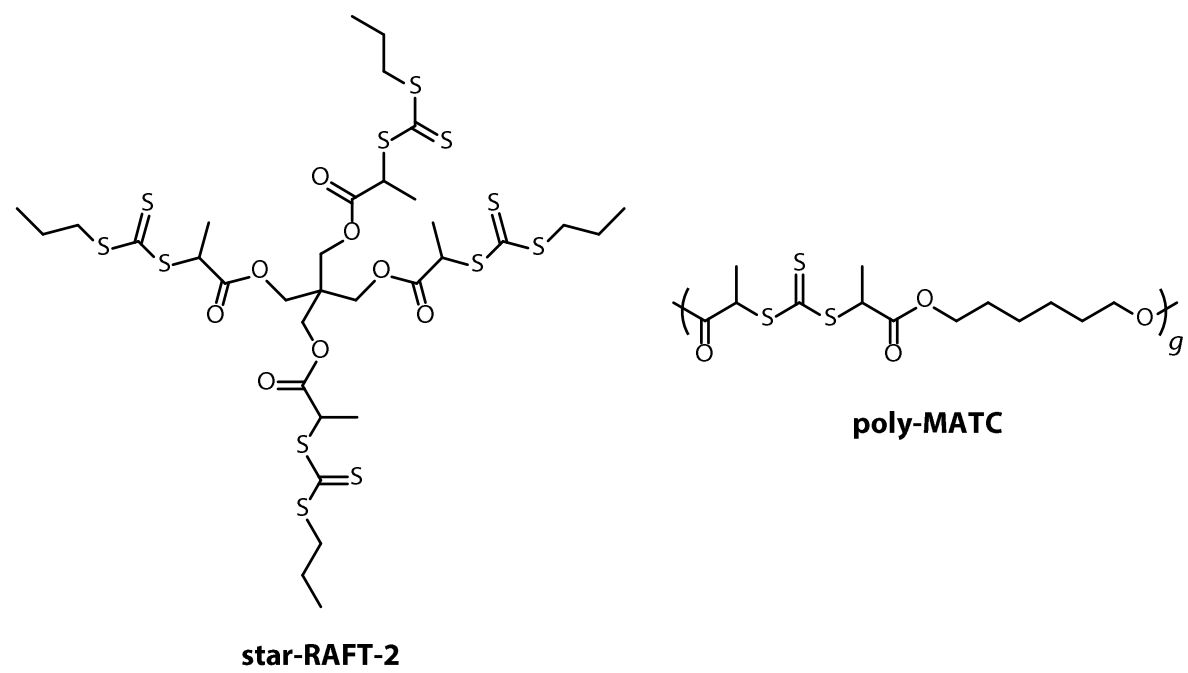

Figure 4-20 Utilized star RAFT agent star-RAFT-2 and polyfunctional RAFT agent polyMATC. The star RAFT agent was kindly provided by Christian Roßner.

Such a blending approach has been utilized by Adhikari et al.229-233 to modify the mechanical performance of triblock copolymers by addition of asymmetric star block copolymers. They prepared block copolymers consisting of polystyrene and polybutadiene and observed increased crack resistance ${ }^{229}$ and material toughness. ${ }^{231}$ The blending process resulted in a modification of the microphase morphology due to the different macromolecular architectures of the components, thereby leading to the enhanced mechanical performance.231,234 A key feature of the blending of chemically identical copolymers is superior compatibility of the components compared with homopolymer blends. Therefore, material properties such as optical transparency are retained in the final polymer material.229 Recently, Shi et al.235-237 refined the strategy by utilizing star copolymers of polystyrene and polyisoprene (PI), exhibiting an asymmetric miktoarm star structure (PS- $b$-(PI- $b$-PS) $)_{3}$ ). Utilizing these miktoarm star copolymers, very high mass contents of polystyrene could be incorporated while keeping microphase morphologies that exhibit ductile deformation behaviour. ${ }^{236,237}$ This was achieved by blending the star copolymers with polystyrene homopolymer. Additionally, this strategy provided a novel microphase morphology which the authors termed "brick-and-mortar" structure. ${ }^{236}$

Here, a novel mixing approach will be introduced that relies on the redistribution mechanism being operative in poly-RAFT-mediated 
polymerizations (see section 2.2). When polyfunctional RAFT agents are combined with star RAFT agents, the redistribution process should yield multiblock star species during polymerization, as illustrated in Scheme 4-4. Then, chemical crosslinks are provided by the star cores, which are beneficial to the mechanical properties. By varying the ratio of linear polyfunctional and star RAFT agent, it is feasible to tailor the number, and therefore the density of crosslinks formed by the star cores. Consequently, issues that occurred in the pure star polymer systems (see chapter 4.3) should be avoided.

To illustrate this mixing concept, model polymerizations of styrene were investigated. Styrene is highly convenient in this respect due to availability of SEC standards that allow for qualitative and quantitative evaluation of results. First, styrene polymerizations were conducted using bi- and polyfunctional RAFT agents DMATC and poly-DMATC (see Figure 3-2). By varying the ratio of bi- and polyfunctional RAFT agent, the redistribution mechanism should allow for a tailored average block number as the ratio of end- and middle blocks is modified. Then, styrene was polymerized in the presence of polyfunctional and star RAFT agent (see Figure 4-20) to obtain fundamental insight into the influence of a different RAFT agent topology on the polymerization process. Finally, the mixing approach was used to prepare poly(styrene-b-butyl acrylate) copolymer materials in the presence of different polyfunctional and star RAFT agent ratios. The synthesized copolymer materials were consequently examined via tensile testing to determine the topological influence on the mechanical properties. In the following sections, the experimental findings will be discussed in detail. 


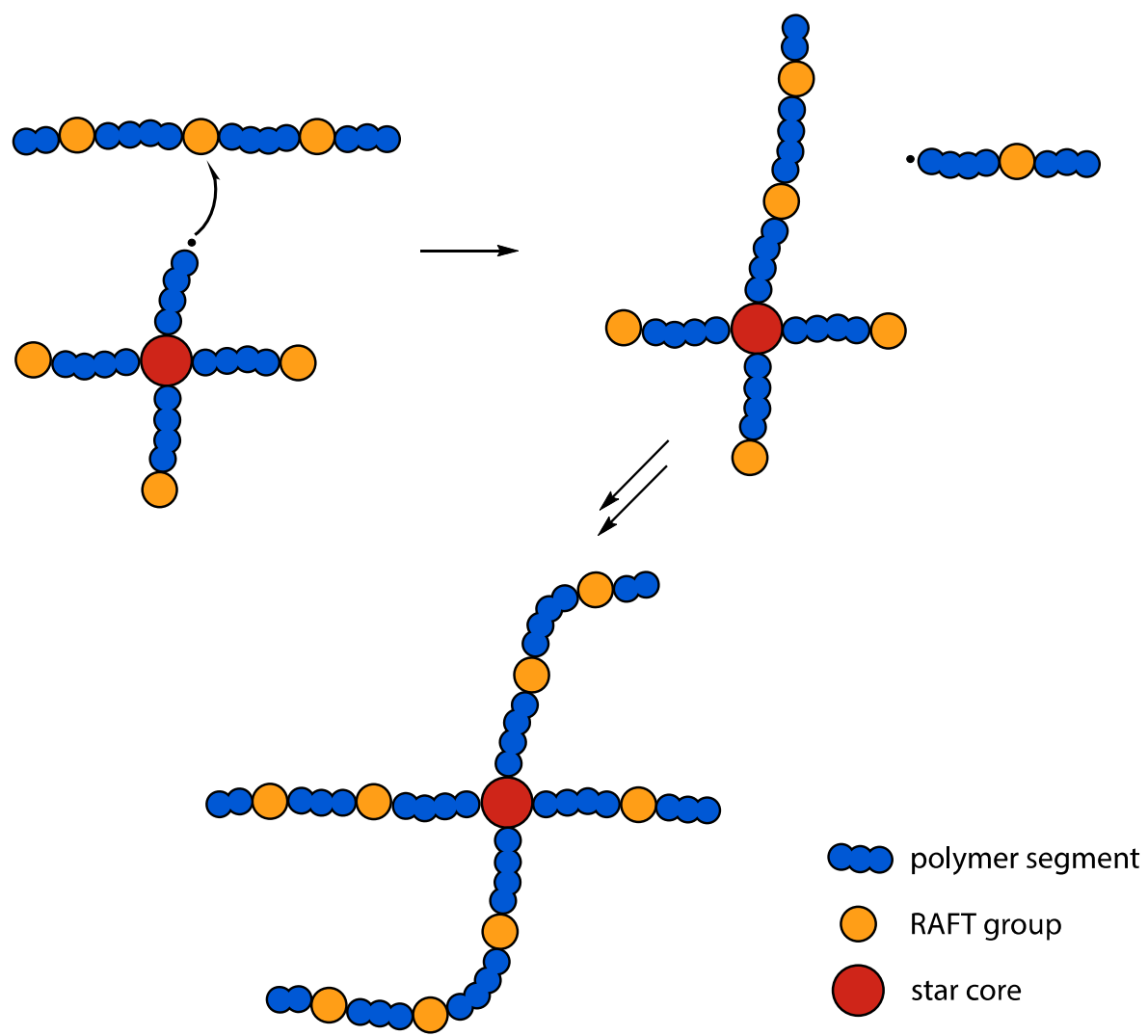

Scheme 4-4 Schematic illustration of the redistribution mechanism when star and polyfunctional RAFT species are simultaneously present. Here, a star-centered radical species adds to a macromolecular species having three RAFT groups along its chain. This reaction yields a new macroradical and a multiblock arm upon the star species. Consecutive reactions lead to the formation of additional multiblock arms and, eventually, a multiblock star molecule. Every blue polymer segment may be an ABblockcopolymer segment when initially formed macro-RAFT species are utlized in a copolymerization. Note that the number of blocks per arm differ due to the redistribution mechanism. 


\subsubsection{Mixing of poly- and bifunctional RAFT agents}

In this section, basic aspects of the mixing concept will be elucidated by discussing a model system of linear poly- and bifunctional RAFT agents. When polyfunctional RAFT agents are applied, they inherently provide two distinct block species - end and middle blocks - to the polymerization system which may not be interconverted (see section 2.2). As the redistribution mechanism shuffles these block species among the macromolecular chains, ${ }^{10}$ tuning the ratio of the respective blocks allows for tailoring of the average number of TTC groups per macromolecule, that is, the average block number. Increasing the fraction of end blocks, the number of blocks per chain decreases and vice versa. It has to be noted that middle blocks may in principle be converted to end blocks via termination of an active chain end. Therefore, it is necessary to select polymerization conditions such that the number of termination events remains low.

To illustrate the described mechanistic feature of the redistribution process, styrene polymerizations were conducted in the presence of different ratios of bifunctional RAFT agent DMATC and polyfunctional RAFT agent poly-DMATC (see Figure 3-2) and the obtained polymers were investigated via GPC (see Figure 4-21). Utilized RAFT agent ratios and respective GPC data are shown in Table 4-15. Both RAFT agents possess similar R-groups to assure similar polymerization kinetics. The average number of RAFT groups $\bar{g}$, here TTC groups, in the polymer may be calculated using the following equation:

$$
\bar{g}=x_{\text {DMATC }} \cdot g_{\text {DMATC }}+x_{\text {poly-DMATC }} \cdot \bar{g}_{\text {poly-DMATC }} \cdot
$$

The molar ratios of the RAFT agents are denoted by $x_{\text {DMATC }}$ and $x_{\text {poly-DMATC }}$ and the average number of TTC groups per RAFT agent is represented by $g_{\text {DMATC }}$ and $\bar{g}_{\text {poly-DMATC }}$, with $g_{\text {DMATC }}$ corresponding to 1 . It has to be noted, that the term TTC group may be used interchangeably with block (see section 2.2 and equation (2.3)) to characterize the resulting macromolecules. ${ }^{10}$ Throughout this text, TTC group will be unambiguously used as term to characterize macromolecular topologies. 
4 Tuning the mechanical properties of biomimetic copolymers via crosslinking

Table 4-15 Bulk styrene polymerizations with different ratios of bifunctional RAFT agent DMATC of polyfunctional RAFT agent poly-DMATC. Corresponding SE chromatograms are depicted in Figure 4-21. Given are the SEC data for original polymer samples and cleavage products obtained by reaction with excess radicals. SEC was measured versus linear polystyrene standards.

\begin{tabular}{|c|c|c|c|c|c|c|}
\hline \multicolumn{4}{|c|}{ original polymer } & \multicolumn{2}{|c|}{ after cleavage } & \\
\hline sample & $\begin{array}{c}\text { molar } \\
\text { portion } \\
\text { DMATC }\end{array}$ & $\begin{array}{c}\bar{M}_{\mathrm{n}, \mathrm{SEC}} / \\
10^{4} \mathrm{~g} \mathrm{~mol}^{-1}\end{array}$ & 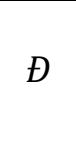 & $\begin{array}{c}\bar{M}_{\mathrm{n}, \mathrm{SEC}} / \\
10^{3} \mathrm{~g} \mathrm{~mol}^{-1}\end{array}$ & $Ð$ & $\bar{g}$ \\
\hline 1 & 1 & 0.5 & 1.2 & 2.8 & 1.39 & 1 \\
\hline 2 & $5 / 6$ & 1.4 & 1.7 & 4.8 & 1.27 & 3.1 \\
\hline 3 & $4 / 6$ & 2.6 & 1.8 & 6.2 & 1.23 & 5.1 \\
\hline 4 & $3 / 6$ & 3.2 & 2.0 & 6.5 & 1.17 & 7.2 \\
\hline 5 & $2 / 6$ & 4.0 & 2.1 & 7.1 & 1.15 & 9.3 \\
\hline 6 & $1 / 6$ & 4.4 & 2.3 & 7.2 & 1.16 & 11.3 \\
\hline 7 & 0 & 4.4 & 2.6 & 7.6 & 1.15 & 13.4 \\
\hline
\end{tabular}

The average number of TTC groups of the polyfunctional RAFT agent $\bar{g}_{\text {poly-DMATC }}$ can be calculated based on its $\bar{M}_{\mathrm{n}, \mathrm{SEC}}$ in accordance with the work of Liu and Cavicchi, as already discussed in section 3.2.2.81 PolyDMATC that was used in this study yielded a $\bar{M}_{\mathrm{n}, \mathrm{SEC}}=4900 \mathrm{~g} \mathrm{~mol}^{-1}$ according to RI-signal of the SE chromatogram which corresponds to an average number of TTC groups of $\bar{g}_{\text {poly-DMATC }}=13$. Equation (4.2) was utilized to calculate the theoretical average number of TTC groups per macromolecule. Then, polymerizations were conducted, keeping the target degree of polymerization per TTC group constant (300 monomer units of styrene). Therefore, increased molar mass would correlate with an increased block number.

The SEC curves of the polystyrene samples are progressively shifted towards higher molar mass with increasing ratio of polyfunctional RAFT agent. As expected, the polystyrene sample that was prepared only with poly-DMATC (grey curve in Figure 4-21) gives rise to the highest molar mass species, that is, consisting of the largest number of blocks. Additionally, the experimentally determined dispersity values follow a trend that agrees well with the theoretical model that can be used to describe the redistribution mechanism (see section 2.2).10 With increasing average block number, an increasing dispersity is expected 
due to the redistribution mechanism. In Figure 4-22 the experimentally determined dispersities are plotted versus the theoretical average number of TTC groups. It can be seen that the experimentally determined dispersities are always larger than the ideal. As the theoretical model only proposes one radical species in the polymerization system that shuffles the TTC groups among macromolecules, neglecting chain-growth or termination events, ${ }^{10}$ this observation may easily be explained. The occurrence of termination events and inherent dispersity of the single blocks both contribute to a broadening of the experimentally determined molar mass distribution.

All polystyrene samples were cleaved at the TTC groups via excess radicals to judge the control of the RAFT process over the individual block lengths. Using excess radicals, oxidative coupling of thiol groups may be avoided that form during the cleavage process. ${ }^{238}$ Investigation of the cleavage products via SEC suggests an effective control, as the dispersities of the products are below 1.5 (see Table 4-15). Furthermore, dispersities follow a decreasing trend while the number average molar masses increase with increasing number of TTC groups. Figure 4-23 further illustrates these findings by comparing selected SEC curves. Both observations may be explained again by comparing the ratio of end and middle blocks. An average number of 13.4 TTC groups was calculated for poly-DMATC, that is, the absolute number of middle blocks exceeds the number of end blocks by a factor of almost 6 and consequently the cleavage product predominantly resembles the molar mass distribution of middle blocks. Since these middle blocks may have a larger probability to grow (see section 2.2 and Ebeling et al. ${ }^{10}$ ) they are on average longer than end blocks. These findings agree well with an earlier study of Ebeling and Vana. ${ }^{50}$ Dispersities decrease because the ratio of middle to end blocks changes in favor of the middle blocks and, consequently, the impact of the end blocks on the molar mass distribution decreases. End blocks feature larger dispersities because they can only grow from one side as opposed to middle blocks that can grow from two sides.

The presented experiments demonstrate that the approach of mixing poly- and bifunctional RAFT agents may be successfully used to tailor the average block number of multiblock polymers. For linear polymers it should be considered as a proof of principle, since the addition of bifunctional RAFT agent reduces the average block number, which is undesirable with regard to mechanical properties as already discussed earlier (see chapter 3). However, as outlined in the introduction of this section, the presented approach should yield materials with interesting 
4 Tuning the mechanical properties of biomimetic copolymers via crosslinking

mechanical properties when star RAFT agents are utilized instead of bifunctional RAFT agents (see Scheme 4-4). In the following section, basic considerations of the mixing process of star and polyfunctional RAFT agents are discussed based on model styrene polymerizations. Especially, differences compared to the mixing of poly- with bifunctional RAFT agents will be highlighted.

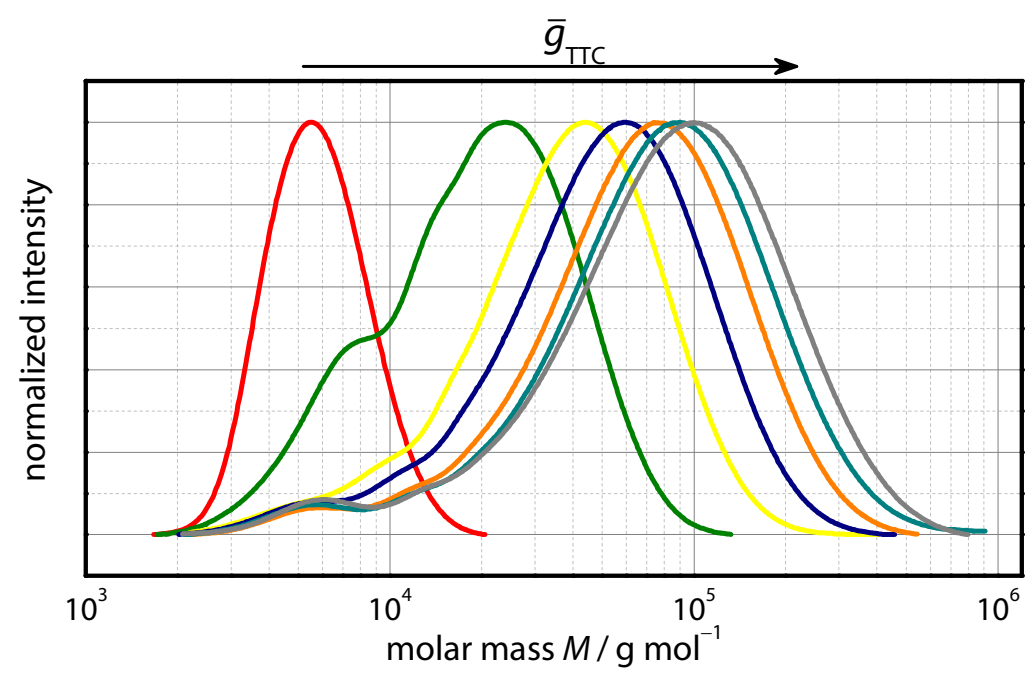

Figure 4-21 SE chromatograms (THF) of polystyrene samples (see Table 4-15) as recorded by RI detection. 


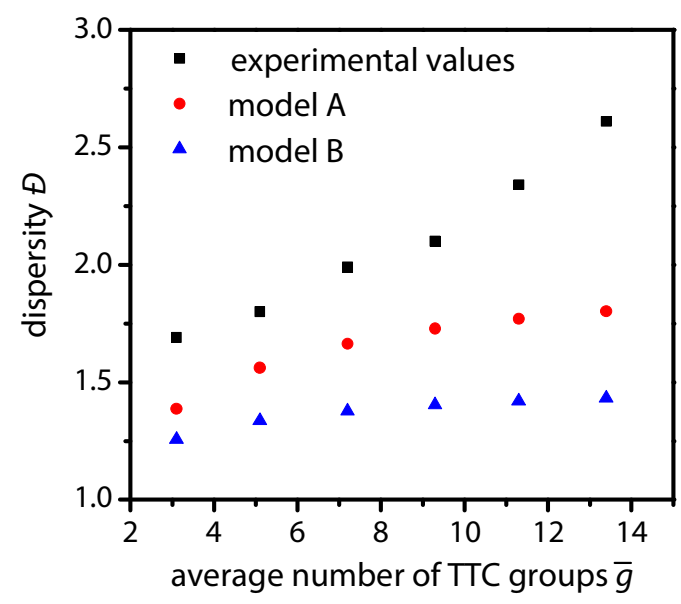

Figure 4-22 Experimental dispersity values (black) and theoretical values that were calculated based on the model introduced by Ebeling et al. ${ }^{10}$ For further explanations see text and section 2.2 .

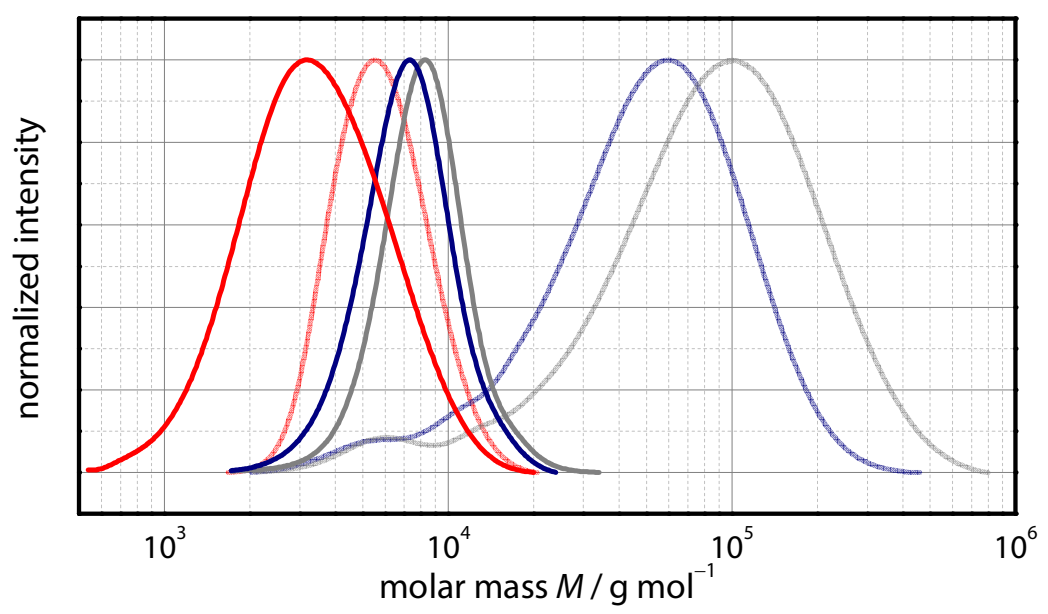

Figure 4-23 SE chromatograms (THF) of polystyrene samples (transparent curves) 1 (red), 4 (blue) and 7 (grey) and corresponding cleavage products (solid curves) as recorded by RI detection (see Table 4-15). The red curve corresponds to pure DMATC, the blue curve to an equal molar ratio of both RAFT agents and the grey curve to pure poly-DMATC. 
4 Tuning the mechanical properties of biomimetic copolymers via crosslinking

\subsubsection{Mixing of polyfunctional and star RAFT agents}

Utilizing star RAFT agents instead of bifunctional linear RAFT agents, a similar mixing process may be achieved when the R-group is formed by the star core. Then, radical species are attached to the star core throughout the course of polymerizations and the core becomes structurally equal to four connected end blocks. This situation is realized in the utilized star RAFT agent star-RAFT-2 that exhibits the same Rgroup as the polyfunctional RAFT agent poly-MATC to assure similar polymerization kinetics (see Figure 4-20). In agreement with the previously discussed mixing of bi- and polyfunctional RAFT agents (see section 4.4.1), styrene polymerizations were conducted with distinct RAFT agent ratios and constant target degree of polymerization per RAFT group, that is, 300 monomer units of styrene. Therefore, the polyfunctional RAFT agent poly-MATC was analysed via SEC to obtain its $\bar{M}_{\mathrm{n}, \mathrm{SEC}}$. In accordance with the analysis of poly-DMATC via SEC (see sections 3.2.2, 4.2.1.2 and 4.4.1), ${ }^{81}$ evaluation of the RI-signal of the SE chromatogram yielded a $\bar{M}_{\mathrm{n}, \mathrm{SEC}}=5300 \mathrm{~g} \mathrm{~mol}^{-1}$ that corresponds to an average number of TTC groups $\bar{g}_{\text {poly-MATC }}=16$. The following equation was used to calculate the average number of TTC groups per macromolecule $\bar{g}$ when star and polyfunctional RAFT agents are applied simultaneously.

$$
\bar{g}=x_{\text {star-RAFT }} \cdot g_{\text {star-RAFT }}+x_{\text {poly-MATC }} \cdot \bar{g}_{\text {poly-MATC }} \cdot
$$

The molar ratios of the RAFT agents are denoted by $x_{\text {star-RAFT }}$ and $x_{\text {poly-MATC }}$ and the average number of TTC groups per RAFT agent is represented by $g_{\text {star-RAFT }}$ and $\bar{g}_{\text {poly-MATC }}$.

Initially, styrene polymerizations were conducted using $0,25,50$ and $75 \mathrm{~mol} \%$ of star-RAFT-2. The prepared polymers were characterized via SEC and the corresponding SE-chromatograms are depicted in Figure 4-24. Mixing star-RAFT-2 with poly-MATC, an extensive broadening of the molar mass distributions is observed. At $75 \mathrm{~mol} \%$ of star-RAFT-2, dispersities of about 4.1 are obtained (see Table 4-16) which renders these polymers comparably ill-defined. Even at $25 \mathrm{~mol} \%$ of star-RAFT-2 a significant broadening and increased dispersity are observed (see Table 4-16). Closer inspection of the SEC curves reavel that both, low and high molar mass species, become more prominent with increasing star RAFT agent ratio compared to polystyrene that was prepared exclusively 
with linear polyfunctional RAFT agent poly-MATC. Low molar mass species are likely to be represented by polystyrene star polymers that possess no additional blocks due to the redistribution process or linear single blocks. High molar mass species presumably result by coupling of star polymers which is a well-known side reaction in R-RAFT star mediated polymerizations. ${ }^{224,225,239}$ However, coupling of star radical species can be a termination process via combination and, therefore, the absolut number of such coupled species should be relatively low under appropriate polymerization conditions. An additional side reaction that could contribute to star-star coupling is transfer to polymer.227 For comparison, a styrene polymerization was conducted in the presence of star-RAFT-2 (black curve in Figure 4-24) and only a minor high molar mass shoulder is observed. This finding suggests that side reactions such as termination or transfer to polymer contribute negligibly to the formation of high molar mass species under the chosen reaction conditions. Since the exchange of RAFT groups is the dominating process compared to termination, it may be assumed that the redistribution process accounts for the observed high molar mass species. It may be readily envisioned that this process leads to coupling of multiple star species which could be considered as a network formation process.

In addition to these experiments, polymerizations were conducted with smaller ratios of star RAFT agent to examine if the mixing approach could also be conducted without significant broadening of the molar mass distributions. Therefore, 1, 5 and $10 \mathrm{~mol} \%$ star-RAFT- 2 were used to mediate styrene polymerizations. The obtained SEC curves are depicted in Figure 4-25 and the respective data are collated in Table 4-16. Under these reaction conditions, no additional broadening is observed and the shape of the molar mass distributions remains essentially unaltered. Based on the presented results it may be assumed that different ratios of star and polyfunctional RAFT agent could lead to materials with distinct mechanical properties. In the next section, the synthesis of corresponding copolymers and investigation of the mechanical properties via tensile testing are discussed. 
Table 4-16 Bulk styrene polymerizations with different ratios of polyfunctional RAFT agent poly-MATC and star RAFT agent star-RAFT-2 (see Figure 4-20). Corresponding SE chromatograms are depicted in Figure 4-24 and Figure 4-25. SEC was measured versus linear polystyrene standards. *Since SEC was measured versus linear polystyrene standards, the experimentally determined molar mass of the star polymer (sample 8) was corrected by a factor of 1.3.226 The factor accounts for the smaller hydrodynamic volume of star polymers compared with linear polymers of the same molar mass.

\begin{tabular}{cccc}
\hline \hline sample & $\begin{array}{c}\text { molar portion } \\
\text { star-RAFT-2 }\end{array}$ & $\begin{array}{c}\bar{M}_{\mathrm{n}, \mathrm{SEC}} / \\
10^{4} \mathrm{~g} \mathrm{~mol}^{-1}\end{array}$ & $Ð$ \\
\hline 1 & $0 \%$ & 2.6 & 2.26 \\
2 & $1 \%$ & 3.6 & 2.14 \\
3 & $5 \%$ & 3.3 & 2.27 \\
4 & $10 \%$ & 3.2 & 2.35 \\
5 & $25 \%$ & 2.7 & 3.27 \\
6 & $50 \%$ & 2.7 & 3.89 \\
7 & $75 \%$ & 3.0 & 4.09 \\
8 & $100 \%$ & $2.7^{*}$ & 1.22 \\
\hline \hline
\end{tabular}

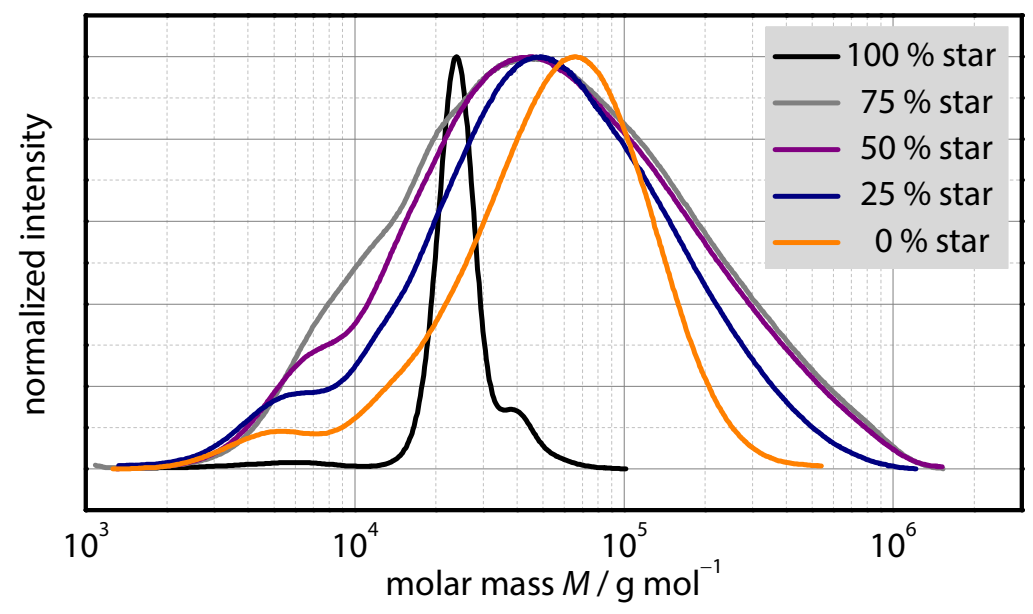

Figure 4-24 SE chromatograms (THF) of polystyrene samples with different molar ratios of polyfunctional RAFT agent poly-MATC and star RAFT agent star-RAFT-2 (see Figure 4-20) as recorded by RI detection. Respective data are collated in Table 4-16 (samples 1, 5, 6, 7 and 8). 


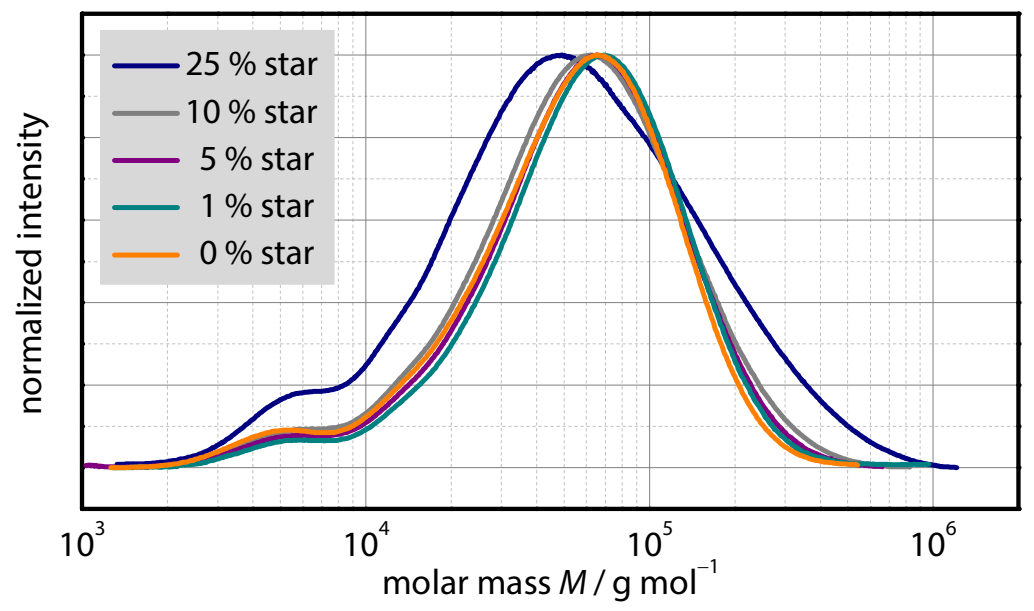

Figure 4-25 SE chromatograms (THF) of polystyrene samples with different molar ratios of polyfunctional RAFT agent poly-MATC and star RAFT agent star-RAFT-2 (see Figure 4-20) as recorded by RI detection. Respective data are collated in Table 4-16 (samples 1, 2, 3, 4 and 5).

\subsubsection{Synthesis of poly(styrene-block-n-butyl acrylate) copolymers}

For the preparation of materials that could be investigated by means of tensile testing, it was decided to use styrene and $n$-butyl acrylate (BA) as comonomers, because the corresponding block copolymers are known to yield samples with dimensional stability. 159 As discussed earlier, this is essential for tensile testing experiments (see section 3.2.1). A further important goal was the synthesis of copolymers that do not differ in comonomer composition or respective block lengths to isolate the topological effect on the mechanical performance. Therefore, polystyrene homopolymer samples with different ratios of polyfunctional and star RAFT agents were prepared first and analysed via SEC to assure comparable number average molar masses. After copolymerization with BA, the samples were investigated by means of NMR to determine the comonomer composition. Additionally, selected samples were examined using AFM to investigate possible influences on the microscopic structure of the samples (see Appendix B). In the final section of this chapter, the results of the tensile testing of the prepared materials is presented. 

crosslinking

\section{Homopolymer synthesis}

In order to achieve a comprehensive understanding of the mixing approach and its influence on the mechanical properties of polymers, various molar ratios of polyfunctional RAFT agent poly-MATC and star RAFT agent star-RAFT-2 (see Figure 4-20) were selected, covering the entire composition range (see Table 4-17). To evaluate the reproducibility of the presented experiments, three polystyrene samples were prepared for $5 \mathrm{~mol} \%$ (star-5i-iii) and $10 \mathrm{~mol} \%$ (star-10i-iii) of star RAFT agent as these ratios did not cause significant broadening of the molar mass distributions (see Figure 4-25). In agreement with preliminary experiments (see previous section) the target degree of polymerization per TTC group was kept constant (300 monomer units of styrene). All samples were characterized by means of SEC (see Table 4-17).

Table 4-17 Overview of polystyrene homopolymer samples that were utilized as macro-RAFT agents for the preparation of copolymers (see Table 4-18). SEC was measured versus linear polystyrene standards. *Since SEC was measured versus linear polystyrene standards, the experimentally determined molar mass of the star polymer (sample 8) was corrected by a factor of 1.3.226 The factor accounts for the smaller hydrodynamic volume of star polymers compared with linear polymers of the same molar mass. For 5 and $10 \mathrm{~mol} \%$ of star-RAFT-2, three samples (denoted by i-iii) were prepared that were subsequently used in copolymerizations with BA (see Table 4-18).

\begin{tabular}{lccc}
\hline \hline sample & $\begin{array}{c}\text { molar portion } \\
\text { of star-RAFT-2 }\end{array}$ & $\begin{array}{c}\bar{M}_{\mathrm{n}, \mathrm{SEC}} / \\
10^{4} \mathrm{~g} \mathrm{~mol}^{-1}\end{array}$ & $Ð$ \\
\hline linear & $0 \%$ & 3.3 & 2.43 \\
star-5i & $5 \%$ & 3.5 & 2.27 \\
star-5ii & $5 \%$ & 3.7 & 2.40 \\
star-5iii & $5 \%$ & 3.5 & 2.38 \\
star-7 & $6.7 \%$ & 3.4 & 2.39 \\
star-8 & $8.3 \%$ & 3.4 & 2.39 \\
star-10i & $10 \%$ & 3.2 & 2.41 \\
star-10ii & $10 \%$ & 3.2 & 2.78 \\
star-10iii & $10 \%$ & 3.5 & 2.55 \\
star-27 & $27 \%$ & 3.4 & 2.89 \\
star-75 & $75 \%$ & 4.6 & 5.00 \\
star-100 & $100 \%$ & $3.0^{*}$ & 1.19 \\
\hline \hline
\end{tabular}


The obtained results agree well with the previously discussed experiments. Especially the determined number average molar masses are remarkably similar for all samples except $75 \mathrm{~mol} \%$ of star-RAFT-2. This sample, again, shows extensive broadening due to the crosslinking of star polymers. One may assume that, based on these results, similar block lengths of polystyrene are realized in all samples, hence rendering them ideal candidates for studies into topological effects.

\section{Copolymer synthesis}

The previously described polystyrene samples were then used as macromolecular RAFT agents in copolymerizations with $n$-butyl acrylate. Comonomer compositions of all samples were determined by NMR spectroscopy. A representative copolymer spectrum is shown in Figure 4-26 for sample LINEAR (see Table 4-18). To calculate the comonomer ratio $r$ (ratio of BA to styrene), the integral of the signal of the aromatic protons of styrene ( $I_{2}$ in Figure 4-26) was normalized to five protons. Then, the following equation may be applied.

$$
r=\frac{0.5 I_{1}}{1+0.5 I_{1}} .
$$

The integral $I_{1}$ corresponds to the two protons of the butyl alkyl chain adjacent to the ester group (see Figure 4-26). The thus obtained comonomer ratios are collated in Table 4-18 and show only minor compositional differences for the copolymer samples. For instance, sample STAR-75 exhibits a slightly lower molar ratio of BA with $42 \mathrm{~mol} \%$ and sample STAR-100 displays a higher molar ratio of BA with $47 \mathrm{~mol} \%$. Therefore, it was decided to utilize the precursor polymers with $5 \mathrm{~mol} \%$ of star RAFT agent (star-5i-iii, see Table 4-17) to prepare copolymers that cover the entire composition range. It is important to note that, despite being relatively small, the different compositions have a significant influence on the mechanical properties as is demonstrated in the next section. Copolymer samples with $10 \mathrm{~mol} \%$ of star RAFT agent (STAR-10i-iii, see Table 4-18) were prepared with similar composition, allowing for an evaluation of the mechanical properties with regard to their reproducibility. Additionally, a conventional blend of the samples LINEAR and STAR-100 was prepared with $10 \mathrm{wt} \%$ of STAR-100, by dissolving the copolymers in toluene and subsequent mixing. The sample BLEND hence constitutes a reference sample that will be compared to STAR-10 with respect to mechanical performance and provides insight into the effectiveness of the novel mixing approach. 
Table 4-18 Overview of poly(styrene- $b$-butyl acrylate) copolymer samples that were characterized via tensile testing. SEC was measured versus linear polystyrene standards in THF. The sample BLEND was prepared by blending polymer sample LINEAR with $10 \mathrm{wt} \%$ of STAR-100. The comonomer ratio $r$ (BA:Styrene) was determined via NMR (see Figure 4-26 and explanations given in the text).

\begin{tabular}{lcccc}
\hline \hline \multirow{2}{*}{ sample } & $\begin{array}{c}\text { molar portion } \\
\text { of star-RAFT-2 }\end{array}$ & $\begin{array}{c}\bar{M}_{\mathrm{n}, \mathrm{SEC}} / \\
10^{4} \mathrm{~g} \mathrm{~mol}^{-1}\end{array}$ & $\emptyset$ & $r$ \\
\hline LINEAR & - & 5.0 & 2.27 & 0.43 \\
STAR-5i & $5.0 \%$ & 4.9 & 2.26 & 0.42 \\
STAR-5ii & $5.0 \%$ & 4.6 & 2.68 & 0.44 \\
STAR-5iii & $5.0 \%$ & 5.1 & 2.46 & 0.47 \\
STAR-7 & $6.7 \%$ & 4.9 & 2.43 & 0.44 \\
STAR-8 & $8.3 \%$ & 4.9 & 2.40 & 0.44 \\
STAR-10i & $10 \%$ & 4.6 & 2.51 & 0.44 \\
STAR-10ii & $10 \%$ & 4.6 & 2.73 & 0.45 \\
STAR-10iii & $10 \%$ & 4.9 & 2.87 & 0.44 \\
STAR-27 & $27 \%$ & 5.1 & 2.67 & 0.45 \\
STAR-75 & $75 \%$ & 6.6 & 4.66 & 0.42 \\
STAR-100 & $100 \%$ & 5.2 & 1.21 & 0.47 \\
\hline \hline
\end{tabular}




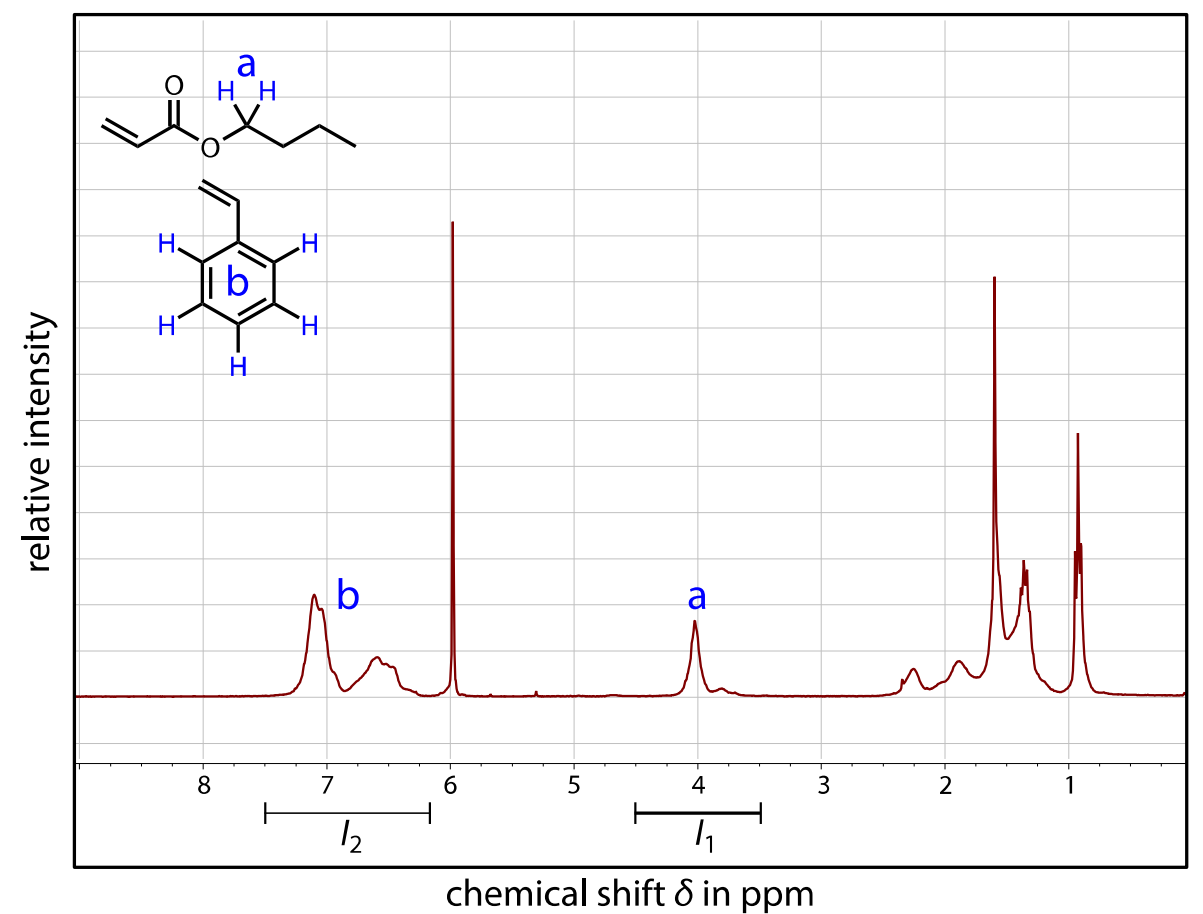

Figure 4-26 ${ }^{1}$ H-NMR spectrum of sample LINEAR in 1,1,2,2-tetrachloroethane-d ${ }^{2}$ with assignment of proton signals (relative intensities scaled arbitrarily). The integrals that

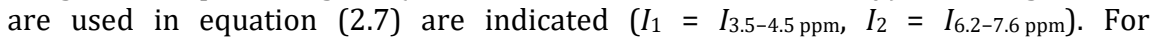
corresponding calculations, integral $I_{2}$ was normalized to five protons.

\subsubsection{Tensile testing}

The mixing process was introduced in order to enhance the mechanical performance of multiblock copolymers that are prepared by means of polyfunctional RAFT agents and to avoid processability issues that occurred in star diblock copolymers with hydrogen bonding segments. Therefore, the results of the tensile testing experiments will be discussed with respect to these two goals. In accordance with other tensile testing experiments presented in this thesis, tensile specimens were prepared via drop-casting. However, the experiments presented in this section could be conducted under improved temperature control. The workshop constructed a casing, that utilizes the air condition of a car and ventilation to cool down the inside of the casing (see Figure 7-1 in the experimental section). Results of the tensile testing are collated in Table 4-19 for all samples. To address the reproducibility of the 

crosslinking

experiments, three copolymer samples with $10 \mathrm{~mol} \%$ of star-RAFT-2 and comparable composition were prepared (STAR-10i-iii, see Table 4-19) and investigated via tensile testing. The averages of the characteristic quantities of the distinct copolymer samples were subsequently averaged and the error was calculated as standard deviation. These values are highlighted as STAR-10 in Table 4-19. As mentioned in the previous section, the samples STAR-5i-iii demonstrate the influence of comonomer composition on the mechanical properties and hence results are shown separately.

First, general remarks on the observed stress-strain behaviour will be given. Similar curves were observed for all copolymer samples except star copolymer STAR-100. Representative stress-strain curves are depicted in Figure 4-27, with STAR-10 being shown exemplarily. The stress-strain behaviour of STAR-10 resembles the biomimetic copolymers discussed in chapter 3 . Yielding is observed after the elastic region, which corresponds to plastic deformation of the tensile specimen. This indicates effective physical crosslinking due to glassy polystyrene domains and entanglements. Afterwards, strain hardening occured that ultimately led to sample failure. While all samples exhibited similar stress-strain responses - excluding STAR-100 - tensile specimens of the copolymer LINEAR displayed strain whitening simultaneously to strain hardening. This finding agrees well with the biomimetic copolymer samples that were also prepared using polyfunctional RAFT agent (see section 3.3), however, the loss of transparency is considered undesirable with regard to applications. ${ }^{145}$ The addition of star RAFT agent offers a significant improvement in this respect because none of the hybrid materials showed strain whitening behaviour. This observation suggests an influence of star species on the deformation mechanism of the bulk materials.

The pure star copolymer STAR-100 shows distinct stress-strain response. After the yield point, strain softening occurs which is associated with stress localization. ${ }^{240}$ Sample failure occurs after significantly smaller strains compared with the other materials under investigation, hence leading to significantly lower material toughness (see Table 4-19). In the following, the determined characteristic quantities, that is, strain at break $\varepsilon_{\text {br, }}$ ultimate tensile strength $\sigma_{\text {UTS, yield }}$ stress $\sigma_{\mathrm{y}}$ and toughness $U_{\mathrm{T}}$ are discussed separately and possible advantages of the mixing approach are elucidated. Therefore, material quantities were plotted versus molar ratios of star RAFT agent starRAFT-2. It has to be noted that, as already desribed above, plotted 
average values and standard deviations for $10 \mathrm{~mol} \%$ star RAFT agent represent three different copolymer samples that were investigated via tensile testing three times each, compared to one copolymer material that has been examined three times for all other samples.

In order to interpret the obtained mechanical properties, one has to consider that the addition of star RAFT agent implies two distinct influences on the macromolecular structures. Addition of star species contributes additional chemical crosslinks to the materials through star cores. This might lead to more homogeneous stress distribution which could explain the absence of strain whitening. However, star-RAFT-2 contributes end blocks to the RAFT process which reduces the average number of trithiocarbonate groups and hence diminishes the average molar mass of the linear matrix polymer. Therefore, both effects influence the mechanical properties adversely and have to be considered in the interpretation of results.

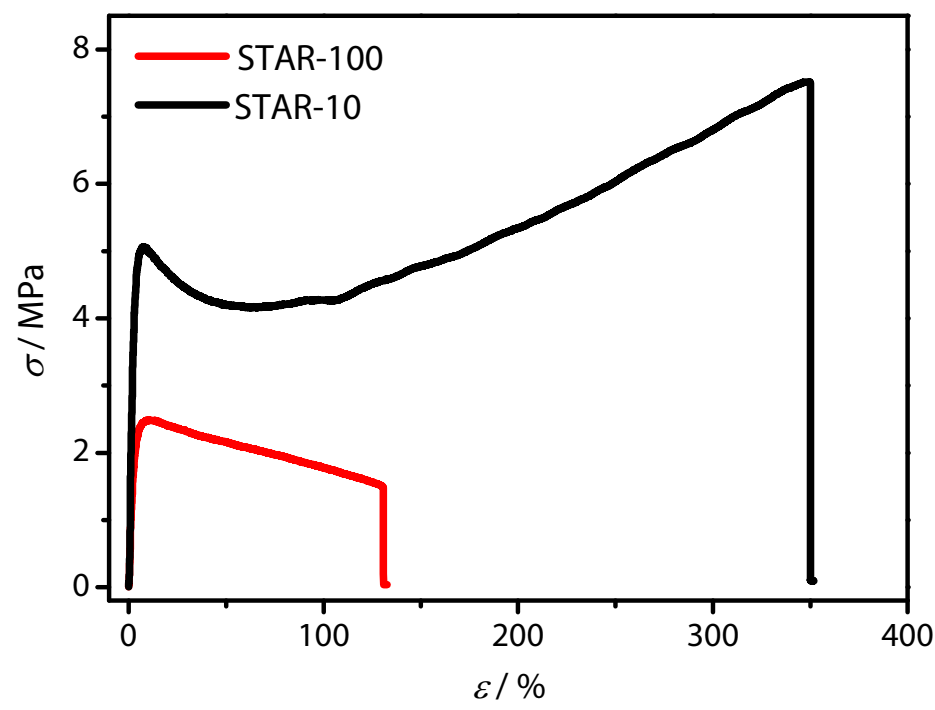

Figure 4-27 Representative stress-strain curves of pure star copolymer STAR-100 and copolymer STAR-10i. The stress-strain curve of the latter is representative for all copolymer samples except STAR-100. 
4 Tuning the mechanical properties of biomimetic copolymers via crosslinking

Table 4-19 Overview of the mechanical properties of tested polymer samples. Characteristic quantities are given as average values and standard deviation that were obtained from three measurements per sample. Yield stress $\sigma_{\mathrm{y}}$ corresponds to the first maximum of the stress-strain curve after the elastic region. STAR-10 represents the calculated average values and standard deviations by averaging over the samples STAR-10i, STAR-10ii, STAR-10iii. Tensile testing experiments were conducted at $18 \pm 1^{\circ} \mathrm{C}$.

\begin{tabular}{lllll}
\hline \hline sample & $\varepsilon_{\mathrm{br}} / \%$ & $\sigma_{\mathrm{UTS}} / \mathrm{MPa}$ & $\sigma_{\mathrm{y}} / \mathrm{MPa}$ & $U_{\mathrm{T}} / \mathrm{MPa}$ \\
\hline LINEAR & $314 \pm 23$ & $5.8 \pm 0.6$ & $3.9 \pm 0.4$ & $13.5 \pm 1.0$ \\
STAR-5i & $299 \pm 11$ & $7.8 \pm 0.4$ & $6.3 \pm 0.4$ & $18.2 \pm 0.8$ \\
STAR-5ii & $366 \pm 8$ & $5.5 \pm 0.8$ & $4.3 \pm 0.5$ & $14.3 \pm 2.1$ \\
STAR-5iii & $501 \pm 28$ & $4.4 \pm 0.5$ & $1.7 \pm 0.3$ & $13.6 \pm 1.8$ \\
STAR-7 & $331 \pm 35$ & $6.2 \pm 0.7$ & $3.8 \pm 0.5$ & $14.5 \pm 2.0$ \\
STAR-8 & $363 \pm 22$ & $6.8 \pm 0.5$ & $3.9 \pm 0.6$ & $17.2 \pm 1.4$ \\
STAR-10i & $340 \pm 33$ & $7.5 \pm 0.8$ & $4.8 \pm 0.2$ & $18.3 \pm 2.7$ \\
STAR-10ii & $319 \pm 24$ & $6.8 \pm 0.1$ & $4.9 \pm 0.8$ & $15.9 \pm 1.2$ \\
STAR-10iii & $336 \pm 7$ & $6.3 \pm 0.2$ & $5.8 \pm 0.6$ & $15.7 \pm 0.7$ \\
\hline STAR-10 & $331 \pm 11$ & $6.9 \pm 0.6$ & $5.1 \pm 0.4$ & $16.6 \pm 1.4$ \\
\hline STAR-27 & $379 \pm 27$ & $5.8 \pm 0.7$ & $4.1 \pm 0.7$ & $15.2 \pm 1.4$ \\
STAR-75 & $260 \pm 27$ & $7.9 \pm 0.7$ & $8.4 \pm 1.9$ & $17.1 \pm 2.6$ \\
STAR-100 & $118 \pm 11$ & $1.5 \pm 0.04$ & $2.6 \pm 0.2$ & $2.4 \pm 0.2$ \\
BLEND & $299 \pm 23$ & $3.8 \pm 0.5$ & $3.3 \pm 0.5$ & $9.2 \pm 1.3$ \\
\hline \hline
\end{tabular}

\section{Strain at break}

Especially in the regime of lower star RAFT agent contents no obvious trend of the determined strain at break values is present (see Figure 4-28 and Table 4-19). Including the margin of error, the samples STAR-5ii, STAR-8, STAR-27 and STAR-75 show significantly different strain at break values compared to copolymer LINEAR. Copolymer STAR-75 displays significantly reduced strain at break compared to copolymer LINEAR which might be explained by the larger dispersity of the copolymer (see Table 4-18) which was interpreted as a crosslinking reaction between star molecules. The material therefore exhibits higher crosslink density, similar to a covalent network, which reduces the strain at break. Such an interpretation may be supported by the observation, that preparation of homogeneous specimens via solvent casting was highly challenging. Macromolecules are less prone to rearrange in the 
thermal annealing process and solvent evaporation is consequently hampered. Such difficulties could not be noticed for any of the other copolymer samples. Significantly increased strain at break values were determined for copolymer samples STAR-5ii, STAR-8 and STAR-27 compared to copolymer LINEAR. No significant change in strain at break can be determined for copolymers STAR-7 and STAR-10.

Comparing the hybrid copolymer STAR-10 with the conventional blend, a significant, albeit small, improvement of strain at break is observed. Still, the novel mixing approach may be considered superior with respect to strain at break and, especially at higher star RAFT agent contents (STAR-27 and STAR-75), relatively large changes of strain at break compared to copolymer LINEAR occur.

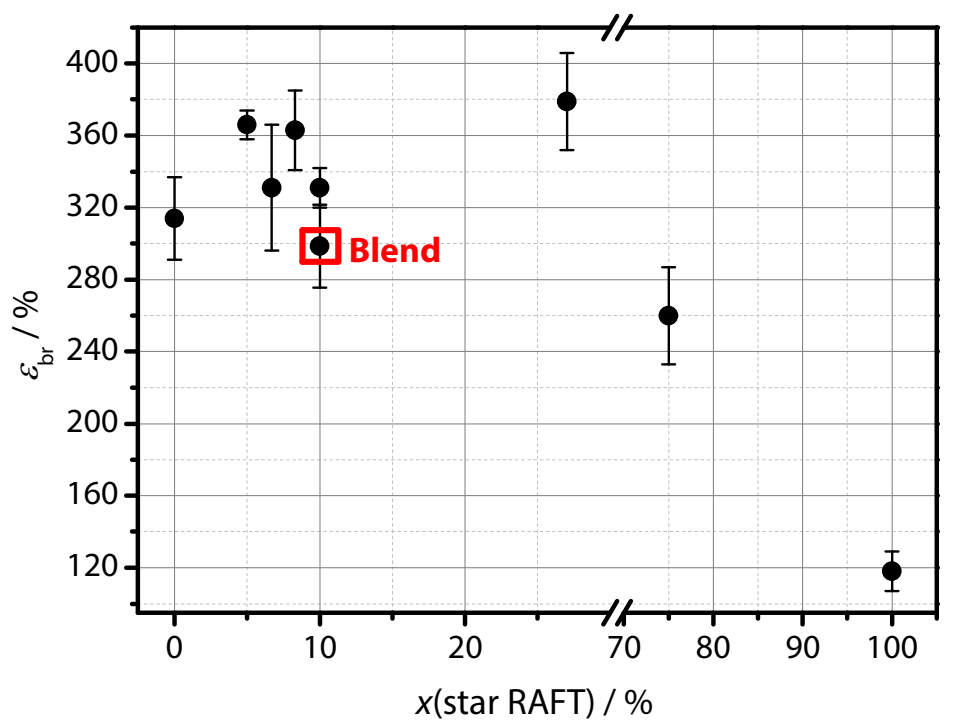

Figure 4-28 Plot of strain at break versus molar star RAFT agent content (see Table 4-19). The conventional polymer blend (BLEND) is highlighted in the graphical representation to facilitate comparison with the hybrid polymer STAR-10. For $5 \%$ and $10 \%$ star content the values of the samples STAR-5ii and STAR-10 are shown as is noted in Table 4-19. 


\section{Ultimate tensile strength}

Regarding ultimate tensile strength, comparable (STAR-5ii, STAR-7 and STAR-27) or larger values were observed after the addition of star RAFT agent to the polymerization process (see Figure 4-29 and Table 4-19). Significantly larger values were determined for the copolymer samples STAR-8, STAR-10 and STAR-75. For the latter, this observation supports the interpretation of a higher crosslink density which leads to the largest ultimate tensile strength of all investigated materials. Based on the reasoning that addition of star RAFT agent provides chemical crosslinks but reduces the average number of blocks, samples STAR-8 and STAR-10 might show an optimum ratio of star and polyfunctional RAFT where the increased number of end blocks does not overrule the increased number of crosslinks. Concerning the investigated RAFT agent ratios it would be desirable for future work to prepare two additional samples with star RAFT ratios between 10 and $27 \mathrm{~mol} \%$ to achieve better understanding of this effect. One would expect a maximum value for the ultimate tensile strength due to the two contradictory effects explained above.

Comparing the samples STAR-10 and BLEND, again, the hybrid materials shows significantly larger ultimate strength. More precisely, the new mixing concept seems to provide synergetic effect with respect to the ultimate tensile strength, whereas the conventional blend appears to resemble an average value of the single components. Such an observation agrees well with literature studies on blends of linear and star block copolymers. ${ }^{231,234,241}$ 


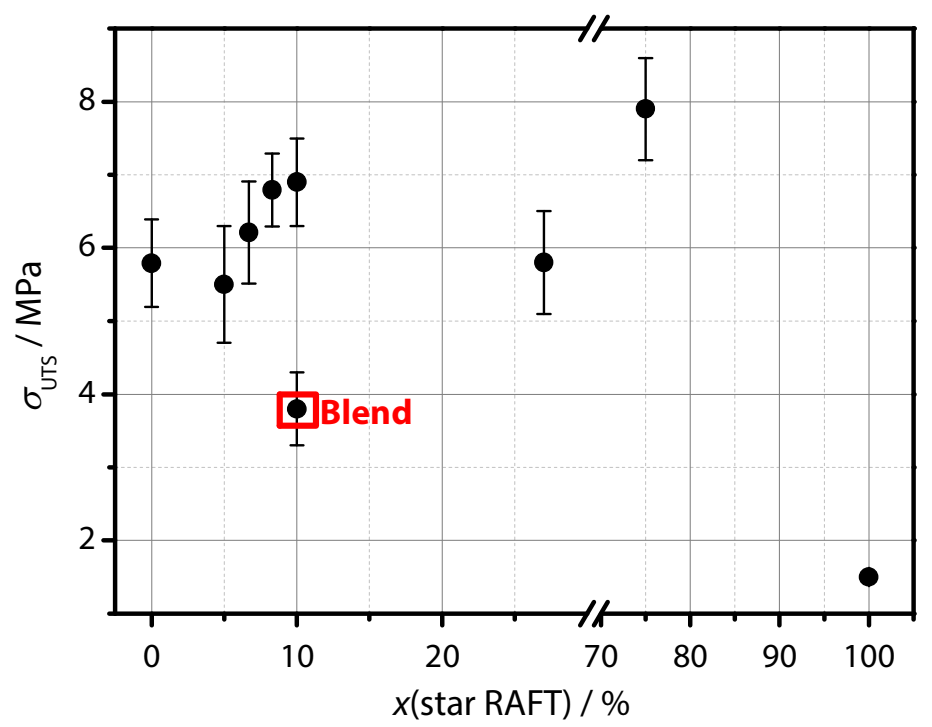

Figure 4-29 Plot of ultimate tensile strength versus molar star RAFT agent content (see Table 4-19). The conventional polymer blend (BLEND) is highlighted in the graphical representation to facilitate comparison with the hybrid polymer STAR-10. Note that the error bar for STAR-100 is too small to be visible in this representation. For $5 \%$ and $10 \%$ star content the values of the samples STAR-5ii and STAR-10 are shown as is noted in Table 4-19.

\section{Yield stress}

The yield point corresponds to the first maximum of the stress-strain curve and characterizes the onset of plastic deformation in a tensile specimen. Therefore, yield stress may be considered as the resistance of a material to initial plastic deformation and hence is correlated with the number or density of effective crosslinks, respectively. The observed yield stress remains essentially unaltered for the samples STAR-5ii, STAR-7, STAR-8 and STAR-27 compared to copolymer LINEAR (see Figure 4-30 and Table 4-19). Significantly increased yield stresses were determined for samples STAR-10 and STAR-75. The former might indicate a beneficial content of star species. Additionally, the hybrid polymer proves to be superior to the conventional polymer blend. Polymer STAR-75 exhibits the largest yield stress which agrees well with the picture of a comparably dense network being formed by crosslinking of star species (see section 4.3). 


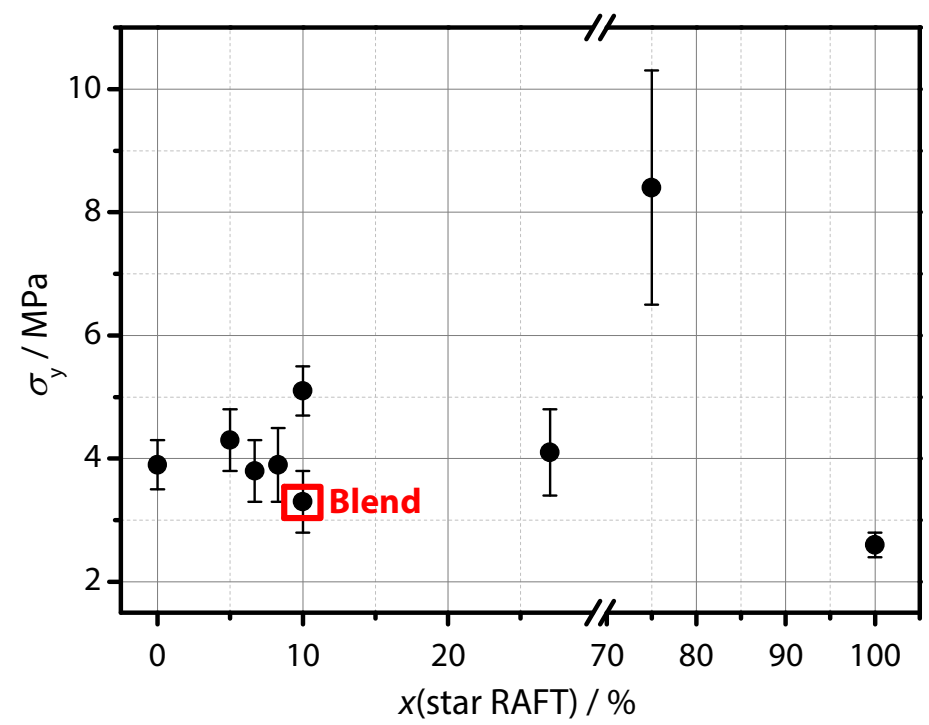

Figure 4-30 Plot of yield stress versus molar star RAFT agent content (compare Table 4-19). The conventional polymer blend (BLEND) is highlighted in the graphical representation to facilitate comparison with the hybrid polymer STAR-10. For $5 \%$ and $10 \%$ star content the values of the samples STAR-5ii and STAR-10 are shown as is noted in Table 4-19.

\section{Toughness}

Material toughness is determined by integrating over the area under the stress-strain curve (see section 2.3). Therefore, the obtained values reflect a combination of the strain at break and ultimate tensile strength values discussed above. It is thus not surprising that the novel hybrid polymers exhibit either similar or larger toughness values than the linear multiblock copolymer LINEAR (see Figure 4-31 and Table 4-19). More importantly, the comparison between copolymer STAR-10 and the conventional blend shows that the determined toughness is almost two times larger for the hybrid polymer. This finding is crucial, as the addition of star species also led to absence of strain whitening in STAR10 , but compared with the conventional blend, synergetic effects with regard to the mechanical performance occur. The observation may also be considered as an indirect proof of the successful mixing of polyfunctional and star RAFT agent, leading to distinct macromolecular structures. 


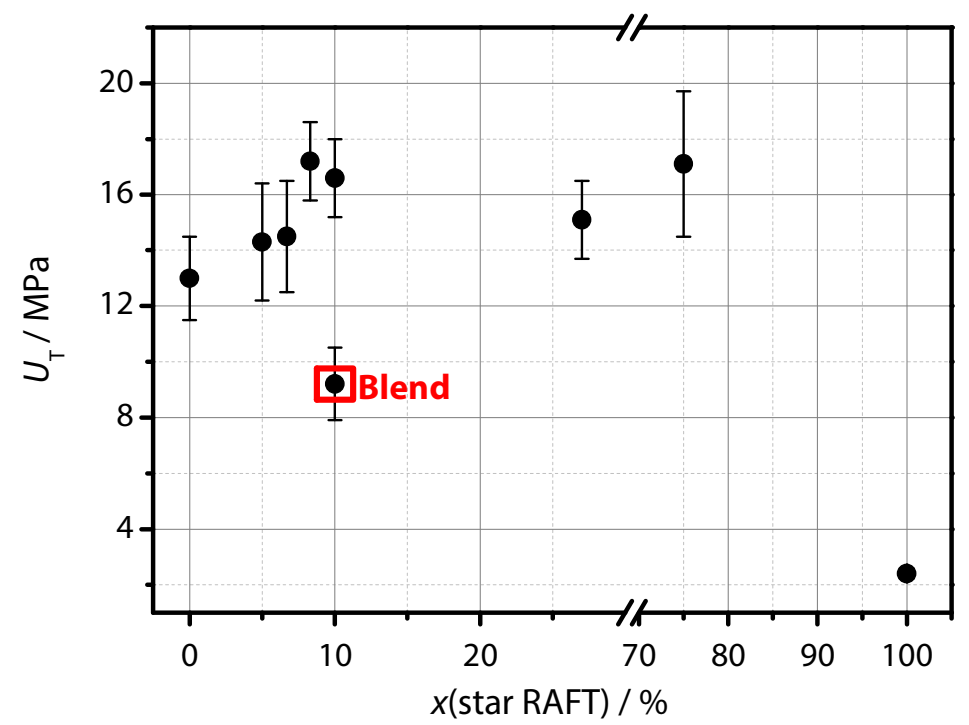

Figure 4-31 Plot of toughness versus molar star RAFT agent content (compare Table 4-19). The conventional polymer blend (BLEND) is highlighted in the graphical representation to facilitate comparison with the hybrid polymer STAR-10. Note that the error bar for STAR-100 is too small to be visible in this representation. For $5 \mathrm{~mol} \%$ and $10 \mathrm{~mol} \%$ star content the values of the samples STAR-5ii and STAR-10 are shown as is noted in Table 4-19.

\section{Compositional effects}

As discussed at the beginning of this section, minor differences in the comonomer composition of the investigated copolymer materials were determined (see Table 4-18). Therefore, three samples with $5 \mathrm{~mol} \%$ star RAFT agent (STAR-5i-iii) covering the entire comonomer composition range ( 42 to $47 \mathrm{~mol} \% \mathrm{BA}$ ) were investigated by tensile testing, to impede misinterpretation of results and exclude composition effects. Representative stress-strain curves for each copolymer are depicted in Figure 4-32. Substantial differences in the stress-strain behaviour are observed with increasing BA content. After the elastic regime, STAR-5i displays a yield point and pronounced strain softening that indicates plastic deformation of the tensile specimen. Increasing the BA content, STAR-5ii shows lower yield stress and less pronounced yielding. At $47 \mathrm{~mol} \% \mathrm{BA}$ content, the yield point almost vanishes and subsequent loading of the tensile specimen leads to continous strain hardening. 

crosslinking

As expected, the strain at break increases with increasing BA content due to longer polymer chains, which has also been observed for the biomimetic multiblock copolymers (see section 3.3). The ultimate tensile strength, consequently, decreases with increasing BA content. These effects result in lower sample toughness of STAR-5ii and STAR-5iii compared to STAR-5i. However, among the copolymers STAR-5ii and STAR-5iii the differences in strain at break and ultimate tensile strength seem to compensate each other. Therefore, one can assume that a BA content of $42 \mathrm{~mol} \%$ would be benficial for a work that aimed to maximize material toughness.

Based on these results, it may be suggested that compositional effects on the mechanical properties are effective for STAR-75 as the sample also exhibits a BA content of $42 \mathrm{~mol} \%$. The observed reduction of strain at break and significantly increased ultimate tensile strength therefore occur due to two factors: crosslinking between star species and lower BA content. Concerning the other copolymer samples under investigation, compositional effects are likely to be excluded as BA content varied between 44 and $45 \mathrm{~mol} \%$.

\section{Reproducibility}

In order to examine the reproducibility of the presented strategy, three copolymers were prepared with $10 \mathrm{~mol} \%$ of star RAFT agent (STAR-10i-iii) and investigated via tensile testing. The characteristic mechanical quantities were determined for three samples of each copolymer and then averaged, calculating the error as standard deviation. Therefore, compared to the samples LINEAR and BLEND, it could be clearly demonstrated that the new approach yields significantly improved mechanical properties with respect to all quantities under investigation, that is strain at break, ultimate tensile strength, yield stress and toughness. In addition, the mixing approach can be used to prevent strain whitening, which was observed in purely linear, biomimetic multiblock copolymers (see chapter 3) and star diblock copolymers (see section 4.3). 


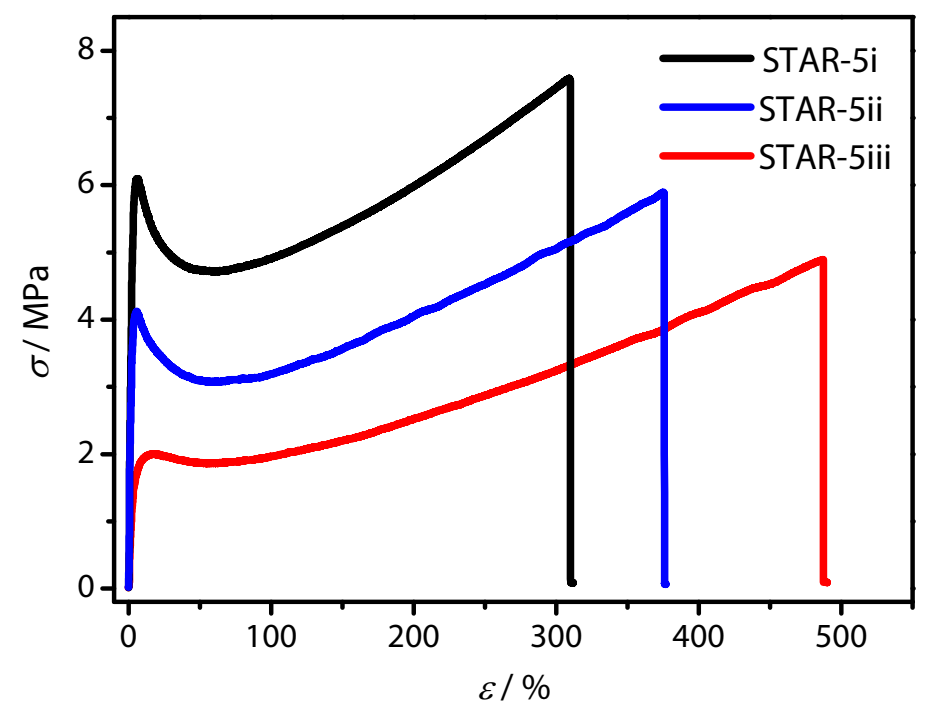

Figure 4-32 Representative stress-strain curves of copolymers STAR-5i, STAR-5ii, and STAR-5iii with a BA content of 42, 44 and 47 mol\%, respectively (see Table 4-19).

\subsubsection{Conclusions}

A new approach for the preparation of multiblock copolymers via polyfunctional RAFT agents was introduced. In order to provide a strategy that can be used to improve the mechanical performance of presented biomimetic multiblock copolymers (see chapter 3), star RAFT agent and polyfunctional RAFT agent were applied simultaneously in the polymerization process. In addition, processability issues should be avoided that occured in biomimetic star diblock copolymers, presumably due to network formation (see section 4.3).

The new strategy relies on the redistribution mechanism being operative in RAFT polymerizations with polyfunctional RAFT agents. To demonstrate the effect of the mixing concept, bi- and polyfunctional RAFT agents were applied in specific ratios to mediate RAFT polymerizations of styrene. It was shown that the resulting average number of TTC groups in the polymers could be tuned by variation of the RAFT agent ratio. Exemplarily, a 4-arm star RAFT agent was selected to be combined with a polyfunctional RAFT agent and poly(styrene-block$n$-butyl acrylate) copolymers were prepared with different RAFT agent 
ratios. Characterization of the as-prepared block copolymers demonstrated that mechanical properties such as ultimate tensile strength and toughness can be enhanced compared to linear multiblock copolymers.

More importantly, the mixing approach proved to be superior compared to conventional blending of linear multiblock copolymer and star block copolymers that were prepared separately. The new hybrid materials exhibit synergetic effects with regard to mechanical properties that are absent in the conventional blend. Both strategies led to prevention of strain whitening that occured in purely linear, multiblock copolymers. Given the experimental ease of the mixing strategy, this is an intriguing finding as it renders the new materials highly attractive for applications.

With increasing star RAFT agent ratio, increasing dispersities were determined that are presumably a result of coupling reactions between star polymers. This effect caused significant issues in the preparation of homogeneous tensile specimens at $75 \mathrm{~mol} \%$ star RAFT content due to extensive void formation. Such processability problems were absent at contents of about $10 \mathrm{~mol} \%$ and, furthermore, no additional broadening of molar mass distributions was observed at these star RAFT contents.

To enhance the mechanical performance of the biomimetic multiblock copolymer that were presented in chapter 3 , it might be particularly useful to apply low star RAFT agent ratios, in order to prevent processability issues that were encountered in corresponding star diblock copolymers (see section 4.3). Further improvement of the mechanical properties might be obtained by use of, for instance, 6-arm star RAFT agents. It has been demonstrated that 6-arm star polymers exhibit improved molecular bridge formation, ${ }^{171}$ which results in better stress distribution and hence larger strength and toughness. Nonetheless, the presented results could clearly demonstrate the effectiveness of the mixing approach. Superior mechanical performance and the prevention of strain whitening could be obtained without changing the required experimental effort. 


\section{5}

\section{Future perspectives}

In section 4.3, spherical silica nanoparticles were used in order to improve the mechanical properties of biomimetic multiblock copolymers (see chapter 3 ). In recent years, however, interest has been growing in anisotropic filler materials such as carbon nanotubes and layered silicates, that is, clay platelets. ${ }^{174}$ These filler materials are promising to improve properties such as mechanical properties, thermal stability, flame retardancy or barrier properties of nanocomposites at even lower loadings than conventional spherical particles. ${ }^{174,242,243}$ The reason for that lies in their beneficial surface-to-volume ratio, which increases the number of interfacial interactions per volume of filler. ${ }^{178}$

Clay in particular has experienced a great deal of attention by scientists. ${ }^{242}$ It is an excellent candidate for the reinforcement of polymers as it is easily available and cheap. To achieve an optimum effect of clay platelets on the mechanical properties, however, thorough dispersion in the matrix polymer is crucial.242,243 When the platelets are well dispersed, the clay is considered to be exfoliated. The preparation of exfoliated structures hence has been a major concern of studies into clay nanocomposites. ${ }^{243} \mathrm{An}$ interesting method is the in situ polymerization via RDRP techniques, as it may offer control over the polymer architecture and the dispersion state of the clay. ${ }^{244}$ The group of Devon A. Shipp contributed to this field by implementation of ATRP244-246 and RAFT247 polymerization approaches that led to well-dispersed structures of clay in polystyrene, poly(methyl methacrylate) and poly $(n-$ 
butyl acrylate). The RAFT polymerization approach appears highly interesting to be adapted using polyfunctional RAFT agents, as it could be demonstrated in this work that thus produced multiblock copolymers exhibit superior mechanical performance compared with triblock copolymers (see chapter 3). The preparation of nanocomposites containing multiblock copolymers and clay should therefore yield materials with exquisite mechanical performance.

The following experiments were conducted at Clarkson University under the supervision of professor Devon A. Shipp. It was examined if polyfunctional RAFT agents could also be applied to yield exfoliated polystyrene-clay nanocomposites. Such nanocomposites would then be promising for further studies. A similar proceeding was followed as reported by Salem and Shipp in 2005 (see Scheme 5-1). ${ }^{247}$ They obtained exfoliated structures at $3 \mathrm{wt} \%$ clay and an average molar mass of $\bar{M}_{\mathrm{n}}=25000 \mathrm{~g} \mathrm{~mol}^{-1}$ for polystyrene. The study used a monofunctional RAFT agent.

For the investigations in professor Shipp's laboratory, organically modified VB16-MMT was still available from earlier studies. Its integrity was checked by means of TGA and X-ray diffraction (XRD). As is shown in Scheme 5-1, the pristine clay has to be modified in the first step with an organic surfactant (VB16). Montmorillonite (Na-MMT) with sodium counterions was applied in this study, which is the most frequently used clay. ${ }^{242,248}$ It can be delaminated relativly easy due to its comparably low cation exchange capacity and hence low ionic interactions between the clay layers. ${ }^{248}$ The corresponding change in $d$-spacing of the clay layers could be examined via XRD. An increasing $d$-spacing affirms successful intercalation of the VB16, as the surfactant is more sterically demanding. Then, the organically modified clay (VB16-MMT) was used in RAFT polymerizations with styrene, as polystyrene was shown to yield welldispersed nanocomposites. ${ }^{247}$ As polyfunctional RAFT agent, polyDMATC was used which was applied in section 4.4 (average number of TTC groups $\bar{g}=13.4$ ). A degree of polymerization of 300 monomer units styrene per TTC group was targeted, in agreement with the original study. The obtained composite materials were then investigated by transmission electron microscopy (TEM) to evaluate the dispersion state of the clay qualitatively. Prior to TEM, samples had to be prepared via sectioning with an ultra-microtome. This step is considered to be the major obstacle for evaluation of the dispersion state, because thin and uniform sections are required to reveal the morphology reliably. ${ }^{243}$ 


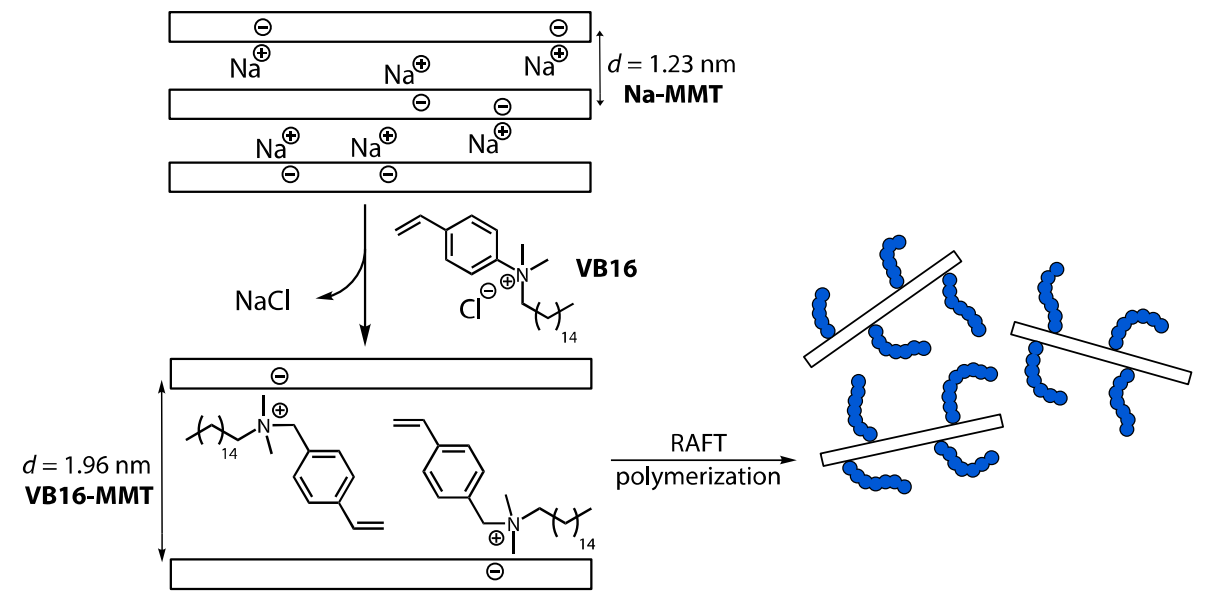

Scheme 5-1 Reaction scheme for the preparation of exfoliated clay nanocomposites via RAFT polymerization. An ion exchange is conducted with the pristine clay (Na-MMT), and a surfactant with a polymerizable group (VB16) is intercalated. Change of the counter ion results in an increased spacing of the clay layers. The RAFT polymerization is conducted in the presence of initiator, monomer and RAFT agent and is not explicitely shown here. Note, that the drawing does not represent the true size ratios of the components. For further explanations refer to the text.

Representative TE micrographs for one section are shown in Figure 5-1. Because of the elemental composition, clay gives good contrast in TEM compared to polystyrene and, therefore, only clay structures are visible. At low magnification (Figure 5-1 a), several darker spots are noticeable which indicate clay layers that could not be dispersed. However, at higher magnifications (Figure 5-1a-c) small stacks exhibiting few layers of clay (intercalated) and even single clay layers (exfoliated) can be seen. This observation agrees well with literature and the original study of Salem and Shipp, because complete exfoliation is seldomly achieved and usually requires more polar monomers. $243,247,248$ Nevertheless, the presented results suggest, that clay may also be dispersed significantly when polyfunctional RAFT agents are applied in the polymerization process. Continuing the presented studies is therefore considered to be highly promising. After reproduction of the obtained results, the next logical step would be the preparation of, for instance, multiblock poly(styrene-block- $n$-butyl acrylate)-clay nanocomposites that could be investigated with respect to their mechanical properties via tensile testing. 

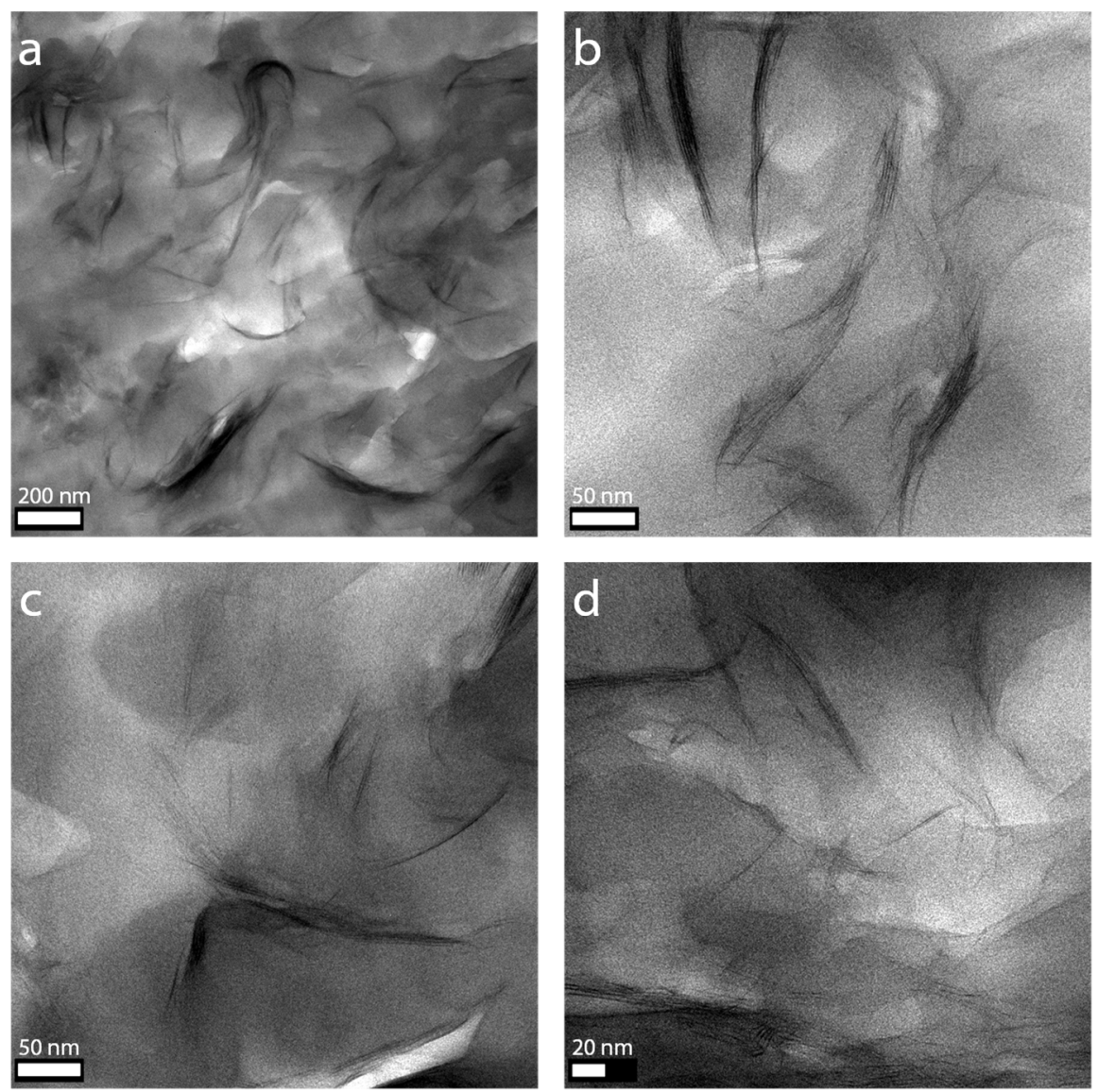

Figure 5-1 TE micrographs of a multiblock polystyrene-clay nanocomposite. Micrographs were taken at different spots of the section and at different magnifications. The respective scale bars are (a) $200 \mathrm{~nm}$, (b) $50 \mathrm{~nm}$, (c) $50 \mathrm{~nm}$ and (d) $20 \mathrm{~nm}$.

Additionally, it could be promising to combine the preparation of clay nanocomposites with the mixing approach of section 4.4. Robello et al. ${ }^{249}$ showed that highly exfoliated polystyrene-clay nanocomposites could be obtained after melt processing, when star shaped polystyrene polymers were utilized. Therefore, a comprehensive study could be conducted using mono-, polyfunctional, and a mixture of polyfunctional and star RAFT agent to prepare polystyrene-clay nanocomposites. Then, the influence on the dispersion state of clay could be investigated systematically with regard to the macromolecular architecture. 
The mixing approach (see section 4.4) itself also offers much potential for further studies. After establishing the concept for 4-arm star RAFT agents, a systematic study on the influence of the arm number on the mechanical properties should be conducted. As already discussed in section 4.4.5, improved molecular bridging is expected with an increased number of arms. ${ }^{171}$ If, for example, 3-, 4- and 6-arm star RAFT agents were applied at similar molar content, one would hence expect a more pronounced impact on the mechanical properties with increasing arm number. Ultimately, adapting the biomimetic polymer design with hydrogen bonding monomer APA might be promising, as the additional chemical crosslinks could potentially give rise to shape-memory abilities of the polymers. ${ }^{149}$

In quest of a synthetic polymer that could match the properties of spider dragline silk, increasing the average molar mass of the biomimetic multiblock copolymers (see chapter 3) is essential.55,250 In order to obtain high molecular weight polymers under RAFT conditions, RAFT agent concentrations need to be reduced. However, this cannot be done arbitrarily, as significant loss of control will occur eventually.251 To overcome this limitation, polymerizations can be conducted under high pressure, because propogation rate is accelerated and the rate of termination is reduced.61,63 Therefore, polymerizations can be conducted at conventional RAFT agent concentrations and higher average chain lengths are obtained at reasonable levels of control. This is very interesting for polyfunctional RAFT agents, as the multiblock structure should allow for ultra high average molar masses. To date, average molar masses of about $10^{6} \mathrm{~g} \mathrm{~mol}^{-1}$ are reported for specific types of RAFT polymerization. ${ }^{33,252,253}$ However, polyfunctional RAFT agents may allow to surpass this value significantly and hence allow to get closer to the imitation of mechanical properties of spider dragline silk. 



\section{6}

\section{Concluding remarks}

Do polyfunctional RAFT agents offer versatile routes towards biomimetic copolymers with excellent mechanical properties? In the first part of this thesis, the preparation of a model multiblock copolymer with hydrogen bonding domains and amorphous segments was described, which resembled the structure of spider dragline silk. Compared with the compositionally similar triblock copolymers, significantly improved mechanical properties in tensile deformation were determined for the multiblock copolymers, including increased strain at break, ultimate tensile strength and toughness. All copolymer samples could be regenerated after failure via thermal annealing. Surprisingly, superior mechanical performance was observed for both triblock and multiblock copolymers after healing. The sample toughness in particular was tremendously increased, indicating the uptake of a larger amount of energy by the samples before failure.

This observation was consequently interpreted by an increased number of effective hydrogen bonding sites. These can also aid in saving orientational order imposed by the tensile deformation, hence strengthening the samples in following tensile testing runs. Concerning the observed healing properties and consequent reinforcement of the polymer materials one can clearly consider the prepared biomimetic polymers as highly functional materials. Especially with respect to applications, such properties appear to be very desirable. 
In order to widen the scope of the novel materials, three different strategies were examined that could potentially give rise to even better mechanical properties. Initially, nanocomposite materials were prepared by addition of unfunctionalized and polymer-grafted silica nanoparticles. Due to lack of temperature control, systematic interpretation was impeded. The obtained results, nevertheless, suggest that significant strengthening and toughening of the nanocomposites may be achieved compared with the neat polymer. Following a different strategy, the macromolecular architecture was switched from the multiblock copolymer structure to star-shaped diblock copolymers. However, the change in macromolecular architecture was found to be detrimental with regard to characterization and processing of the copolymer materials. It was argued that the chemical crosslinks, provided by the star cores, in addition to the hydrogen bonding segments would lead to network formation which resulted in the aforementioned issues.

To address these problems, a new strategy was developed that relies on the redistribution mechanism of polyfunctional RAFT agents. When polyfunctional RAFT agents are mixed with star RAFT agents, the redistribution process of RAFT groups yields multiblock star copolymers that are smoothly incorporated into the matrix of linear multiblock copolymer chains. By tuning the ratio of star and polyfunctional RAFT agents, the mechanical properties of the resulting copolymers could be altered. At low star RAFT contents, the mechanical properties can even be improved compared with linear multiblock copolymers. Additionally, it could be clearly shown, that the presented strategy yields materials that are superior to conventional polymer blends. The results of this work conclusively show that polyfunctional RAFT agents are an exquisite means for the development of highly functional, highperformance materials. 


\section{Experimental section}

\subsection{Instrumentation}

\subsubsection{Atomic force microscopy}

AFM measurements were performed on a BRUKER MULTIMODE 8 microscope in peak force tapping mode. A ScanAsyst-Air Cantilever (Bruker, nominal resonance frequency $=70 \mathrm{kHz}$, nominal spring constant $=0.4 \mathrm{Nm}^{-1}$, and nominal radius $=2 \mathrm{~nm}$ ) was used. Samples were typically prepared via spin-casting of $40 \mu \mathrm{L}$ polymer solution ( $2 \mathrm{wt} \%$ ) onto a glass substrate. The glass substrates had been cleaned for $30 \mathrm{~min}$ in a ZEPTO plasma cleaner by DIENER ELECTRONIC. As solvents PGMEA (poly(APA-block-methyl acrylate) block copolymers, see chapter 3 and appendix A) and toluene (poly(styrene-block- $n$-butyl acrylate) block copolymers, see section 4.4 and appendix B) were used. Annealing conditions of the samples are indicated in the text of the respective chapters.

\subsubsection{Chromatography}

\subsubsection{Column chromatography}

Column chromatography was performed using silica gel 60 (70-230 mesh, 63-200 $\mu \mathrm{m}$, SIGMA ALDRICH). 


\subsubsection{Size-exclusion chromatography (SEC)}

SEC characterization was performed with THF (flow rate $=1.0 \mathrm{~mL} \mathrm{~min}^{-1}$ ) at $35^{\circ} \mathrm{C}$ as eluent using an AGILENT 1260 InFINITY system. The set-up comprised an isocratic HPLC-pump and an autosampler, a PSS SDV (styrene-divinylbenze copolymer network) guard column $(8 \times 50 \mathrm{~mm})$ and three PSS GRAM separation columns $(8 \times 300 \mathrm{~mm}$, particle size $=$ $10 \mu \mathrm{m}$, pore sizes $=30 \AA 0^{3} \AA \AA$ and $10^{3} \AA$ ) . As detection system a UVdetector (set to a wavelength of $310 \mathrm{~nm}$ ) and an RI detector were used. The system was calibrated using PSS polystyrene standards $\left(M_{\mathrm{P}}=0.5-\right.$ $2520 \mathrm{~kg} \mathrm{~mol}^{-1}$ ) of low dispersity with toluene as the internal standard. Polymer samples were dissolved in THF and filtered through a syringe filter with a $0.45 \mu \mathrm{m}$ porous polytetrafluoroethylene (PTFE) membrane prior to injection. The concentration of the samples was $3 \mathrm{~g} \mathrm{~L}^{-1}$.

SEC characterization was also performed with DMAc containing $0.1 \mathrm{wt} \%$ lithium bromide (flow rate $=0.8 \mathrm{~mL} \mathrm{~min}^{-1}$ ) at $45^{\circ} \mathrm{C}$ as eluent using an AGILENT 1260 INFINITY system. The set-up comprised an isocratic HPLCpump and an autosampler, a PSS GRAM (polyester copolymer network) guard column $(8 \times 50 \mathrm{~mm})$ and three PSS SDV separation columns $\left(8 \times 300 \mathrm{~mm}\right.$, particle size $=10 \mu \mathrm{m}$, pore sizes $=10^{6} \AA, 10^{5} \AA$ and $\left.10^{3} \AA\right)$. As detection system a UV-detector (set to a wavelength of $310 \mathrm{~nm}$ ) and an RI detector were used. The system was calibrated using PSS polystyrene standards $\left(M_{\mathrm{P}}=0.5-2520 \mathrm{~kg} \mathrm{~mol}^{-1}\right)$ of low dispersity with toluene as the internal standard. Polymer samples were dissolved in THF and filtered through a syringe filter with a $0.45 \mu \mathrm{m}$ porous polytetrafluoroethylene (PTFE) membrane prior to injection. The concentration of the samples was $3 \mathrm{~g} \mathrm{~L}^{-1}$.

Polymer samples that contained APA had to be additionally modified prior to characterization via SEC (see section 7.3.8.4).

\subsubsection{Differential scanning calorimetry}

DSC measurements were conducted on a METTLER TOLEDO 820 with a cryostat (LAUDA KRYOMAT RUK 90) in a temperature range from -30 to $200{ }^{\circ} \mathrm{C}$. Measurements were performed with a heating rate of $10^{\circ} \mathrm{C} \mathrm{min}^{-1}$ and a constant nitrogen flow of $4.5 \mathrm{~mL} \mathrm{~min}^{-1}$ to prevent water condensation. 


\subsubsection{Dynamic light scattering}

DLS measurements were performed with a MALVERN ZETASIZER NANO S instrument equipped with a $\mathrm{He}-\mathrm{Ne}$ laser (wavelength $=633 \mathrm{~nm}$ ). The scattered intensity was detected at an angle of $173^{\circ}$. Measurements were conducted to investigate the particle size distribution of silica nanoparticles (see section 4.2). Particles were dispersed in PGMEA either via ultrasonification (particles without polymer shell) or manual shaking (polymer-coated particles) for $30 \mathrm{~min}$ yielding dilute analyte samples. After filtration through a PTFE filter $(0.2 \mu \mathrm{m})$ the dispersions were filled into a glas cuvette and measured three times (a 10 measurements).

\subsubsection{Lyophilisation}

Freeze-drying was performed using a CHRIST ALPHA-2,4-lyophilisator and 1,4-dioxane as solvent.

\subsubsection{Nuclear magnetic resonance (NMR) spectroscopy}

${ }^{1} \mathrm{H}-\mathrm{NMR}$ spectra were measured with a VARIAN UNITY 300 instrument at room temperature using residual solvent proton signals as internal standard. Substance concentrations of approximately $20 \mathrm{~g} \mathrm{~L}^{-1}$ were used.

\subsubsection{Tensile testing}

Tensile testing was performed on a ZWICK \& ROELL Z2.5 tensile testing machine. Measurements were conducted at $21 \pm 1^{\circ} \mathrm{C}$ or at $18 \pm 1{ }^{\circ} \mathrm{C}$ as indicated in the respective section. For measurements at $18{ }^{\circ} \mathrm{C}$ a cooling chamber was used that was constructed by the institute workshop (see Figure 7-1). All measurements were performed with a strain rate of $0.33 \mathrm{~mm} \mathrm{~s}^{-1}$ (20 $\mathrm{mm} \mathrm{min}^{-1}$, strain control). Tensile data reported herein are averages taken from at least three specimens per polymer sample. The data were collected and analysed by the computer program testXpert II, yielding Young's modulus $(E)$, strain at break $\left(\varepsilon_{\max }\right)$ and ultimate tensile strength $\left(\sigma_{\text {UTS }}\right)$ as characteristic quantities. Toughness $\left(U_{\mathrm{T}}\right)$ and yield strength $\left(\sigma_{\mathrm{y}}\right)$ were obtained by evaluating tensile data separately using the program ORIGINPRO $8.5 \mathrm{G}$. 

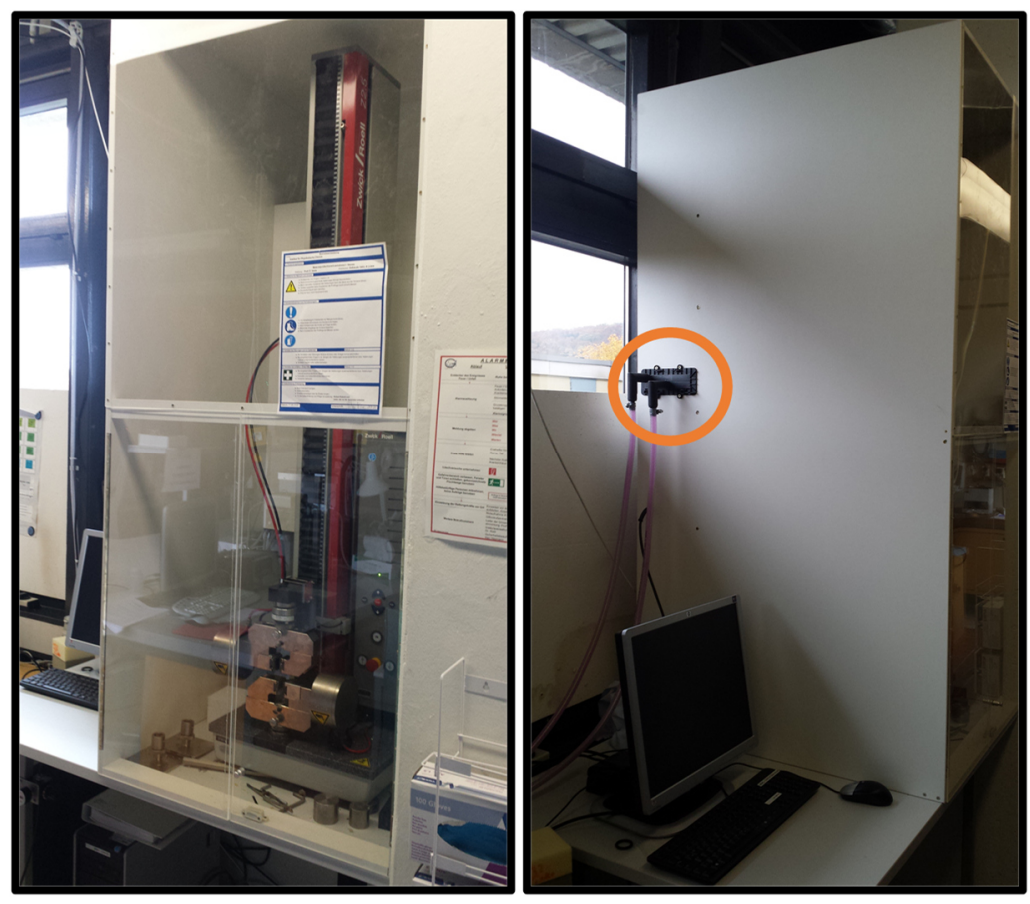

Figure 7-1 Photographs of the cooling casing that was constructed by the workshop. In the right photo, the position of the air conditioning is highlighted. 


\subsubsection{Sample preparation}

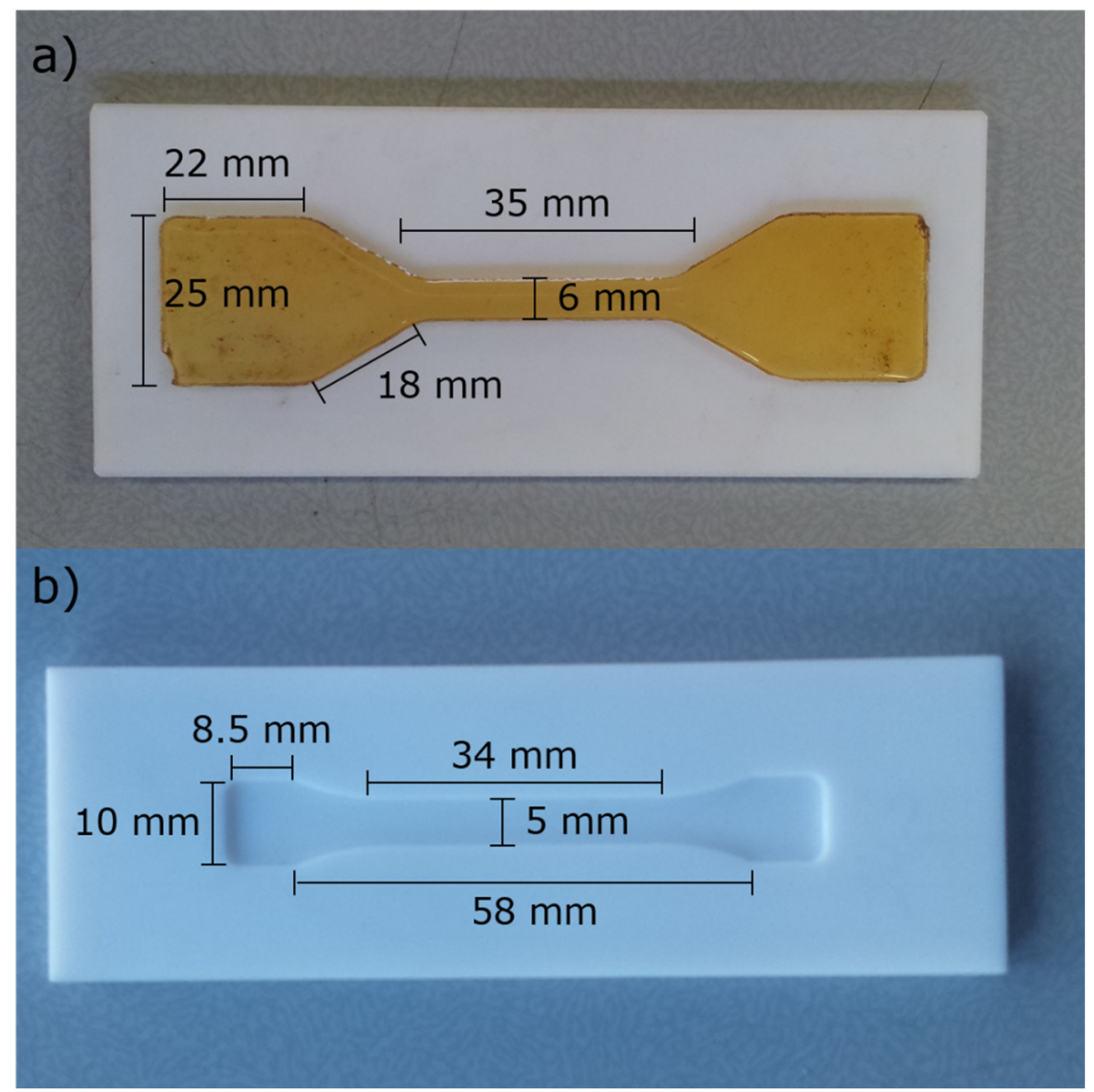

Figure 7-2 Pictures of a) a large tensile specimen according to ISO-37 norm type 1 and b) a small teflon mold according to ISO 527-2 norm type 1BA, including relevant dimensions.

Dogbone shaped polymer films were prepared via drop-casting of polymer solution (33wt\%) into a PTFE mold (see Figure 7-2b). The samples were then dried overnight (approximately $15 \mathrm{~h}$ ) under vacuum at $100{ }^{\circ} \mathrm{C}$. As solvents PGMEA (for poly(APA-block-methyl acrylate) block copolymers, see chapter 3 , sections 4.2 and 4.3) and toluene (for poly(styrene-block-n-butyl acrylate) block copolymers, see section 4.4) were used. 


\subsubsection{Regeneration of poly(APA-block-methyl acrylate) block copolymer samples}

Regeneration of tensile specimens that were strained until failure was conducted in the following manner: the pieces of a broken sample were gently pushed into a PTFE mold and then the mold was put into a vaccum and heated to $100{ }^{\circ} \mathrm{C}$ for at least $12 \mathrm{~h}$. After cooling at room temperature the specimens did not display any visible defects.

\subsubsection{Thermogravimetric analysis}

Thermogravimetric analysis was conducted with a NETZSCH TG 209 F3 Tarsus instrument from room temperature up to $1000^{\circ} \mathrm{C}$ with a heating rate of $10 \mathrm{~K} \mathrm{~min}^{-1}$ under a nitrogen flow rate of $10 \mathrm{~mL} \mathrm{~min}^{-1}$.

\subsection{Commercially acquired chemicals}

Acryloyl chloride (96\%, ABCR), ammonia solution (32\%, VWR), benzyl bromide (98\%, ALDRICH), 2-bromopropionic acid ( $\geq 99$, ALDRICH), carbon disulfide $(\geq 99.9 \%$, SIGMA-ALDRICH), chloroform (HPLC grade, FISHER SCIENTIFIC), cyclohexane ( $\geq 99.0 \%$, SIGMA-ALDRICH), 1,2-dimethoxyethane ( $\geq 99 \%$, SIGMA-ALDRICH), 1,4-dioxane (99.5\%, GRÜSSING), ethanol ( $\geq 99.8 \%$, SigMA-ALDRICH), 1,6-hexanediol (99\%, ALDRICH), hydrazine monohydrate (55\%, ACROS ORGANICS), hydrochloric acid ( $\geq 37 \%$, ALDRICH), L-phenylalanine ( $\geq 98 \%$, SIGMA-ALDRICH), maleic anhydride (99\%, ALDRICH), methanol ( $\geq 99.5 \%$, SIGMA-ALDRICH), 3-methyl-1-( $p$ tolyl)triazene (98\%, SIGMA-ALDRICH), pentaerythritol tetrakis(3mercaptopropionate) ( $>95 \%$, ALDRICH), potassium hydroxide $(\geq 85$, ROTH), potassium phosphate ( $\geq 98 \%$, SIGMA-ALDRICH), propylene glycol monomethyl ether acetate (PGMEA, $\geq 99.5 \%$, SIGMA-ALDRICH), $p$-toluenesulfonic acid monohydrate ( $\geq 98.5 \%$, SIGMA-ALDRICH), tetrabutylammonium hydrogensulfate ( $\geq 99.0 \%$, SigMA-ALDRICH), tetraethyl orthosilicate (98\%, ALDRICH) toluene (p.A. grade, FISHER SCIENTIFIC) were used as received without further purification. Deuterated solvents (chloroform-d, dimethyl sulfoxide- $\mathrm{d}_{6}, 1,1,2,2$ tetrachloroethane- $\mathrm{d}_{2}$ ) for NMR spectroscopy were provided by the NMR laborartory.

\subsubsection{Monomers}

Methyl acrylate (MA, SIGMA-ALDRICH, $99 \%$, St. Louis, MO) was passed through a column of inhibitor remover (Sigma-ALDRICH) prior to use, 
styrene (Sty, $\geq 99 \%$, ALDRICH) and $n$-butyl acrylate (BA, $\geq 99 \%$, ALDRICH) were passed through a column of basic aluminum oxide (basic, Brockmann I, 150 mesh, ALDRICH) before use. All monomers were freshly purified before usage.

\subsubsection{Initiators}

2,2'-Azobis(isobutyronitrile) (AIBN, $\geq 98 \%, \quad$ FLUKA) and 1,1'-Azobis(cyclohexanecarbonitrile) (ACCN, $98 \%$, ALDRICH) were recrystallized from Methanol and stored at $8{ }^{\circ} \mathrm{C}$ before use.

\subsubsection{RAFT agent}

The star RAFT agent pentaerythritol-tetrakis(methyl2 (propylthiocarbonothioylthio)-propanoate) (Star-RAFT-2, see Figure 4-20) was kindly provided by Christian Roßner who has already reported on the synthesis. ${ }^{79}$ Its integrity was checked by NMR spectroscopy before usage.

${ }^{1} \mathrm{H}-\mathrm{NMR} \quad\left(300 \mathrm{MHz}, \quad \mathrm{CDCl}_{3}\right): \quad \delta=4.83 \quad(\mathrm{q}, \quad J=7.4 \mathrm{~Hz}, \quad 4 \mathrm{H}$, $\left.\mathrm{OC}(=0)-\mathrm{CH}\left(\mathrm{CH}_{3}\right) \mathrm{S}-\right), 4.06\left(\mathrm{~m}, 8 \mathrm{H},-\mathrm{C}\left(\mathrm{CH}_{2}\right)-\right), 3.33(\mathrm{~m}, 8 \mathrm{H}$, $\left.-\mathrm{SC}(=\mathrm{S}) \mathrm{S}-\mathrm{CH}_{2}-\mathrm{CH}_{2}-\mathrm{CH}_{3}\right), 1.73\left(\mathrm{~m}, 8 \mathrm{H},-\mathrm{SC}(=\mathrm{S}) \mathrm{S}-\mathrm{CH}_{2}-\mathrm{CH}_{2}-\mathrm{CH}_{3}\right), 1.59$ (d, $\left.J=7.4 \mathrm{~Hz}, 12 \mathrm{H}, \mathrm{OC}(=0)-\mathrm{CH}\left(\mathrm{CH}_{3}\right) \mathrm{S}-\right), 1.02(\mathrm{t}, J=7.4 \mathrm{~Hz}, 12 \mathrm{H}$, $\left.\mathrm{SC}(=\mathrm{S}) \mathrm{S}-\mathrm{CH}_{2}-\mathrm{CH}_{2}-\mathrm{CH}_{3}\right)$.

\subsection{Synthesized substances}

\subsubsection{Synthesis of monomer N-Acryloyl-L-phenylalanine (APA)}<smiles>C=CC(=O)N[C@@H](Cc1ccccc1)C(=O)O</smiles>

Sodium hydroxide (12.4 g, $0.303 \mathrm{~mol}, 2$ eq.) was dissolved under continuous stirring in demineralized water $(75.0 \mathrm{~mL})$ and the solution was cooled using an ice bath. L-phenylalanine $(25.5 \mathrm{~g}, 015 \mathrm{~mol}, 1 \mathrm{eq}$.) was added and stirring continued for $30 \mathrm{~min}$. To the yellow solution acryloyl chloride (12.8 mL, $015 \mathrm{~mol}, 1 \mathrm{eq}$ ) was added over $30 \mathrm{~min}$ and after an 
hour a precipitate formed. Additional demineralized water $(100 \mathrm{~mL})$ was added to redissolve the precipate. Subsequently, the solution was acidified with concentrated hydrochloric acid to $\mathrm{pH}=2$ and the reaction was continued for one hour. The precipitate was washed with dilute hydrochloric acid solution $(1.0 \mathrm{M}, 300 \mathrm{~mL})$ and the crude product was recrystallized twice from ethanol/demineralized water (1:1) to yield white crystals (19.4 g, $88.7 \mathrm{mmol}, 59 \%$ ).

${ }^{1} \mathrm{H}-N M R(300 \mathrm{MHz}$, DMSO-d 6 ): $\delta=12.74(\mathrm{~s}, 1 \mathrm{H},-\mathrm{C}(=0) \mathrm{OH}), 8.42(\mathrm{~d}$, $J=8.5 \mathrm{~Hz}, 1 \mathrm{H},-\mathrm{NH}), 7.39-7.10\left(\mathrm{~m}, 5 \mathrm{H},-\mathrm{CH}_{\mathrm{Ar}}\right), 6.35-6.17(\mathrm{~m}, 1 \mathrm{H}$, $\left.\mathrm{CH}_{2}-\mathrm{CH}-\mathrm{C}(=0) \mathrm{NH}\right), 6.10-5.97\left(\mathrm{~m}, 1 \mathrm{H}, \mathrm{CH}_{2}-\mathrm{CH}-\mathrm{C}(=0) \mathrm{NH}\right), 5.64-5.50$ $\left(\mathrm{m}, 1 \mathrm{H}, \mathrm{CH}_{2}-\mathrm{CH}-\mathrm{C}(=\mathrm{O}) \mathrm{NH}\right), 4.56-4.44(\mathrm{~m}, 1 \mathrm{H}, \mathrm{CH}-\mathrm{C}(=\mathrm{O}) \mathrm{OH}) 2.77-3.16$ (m, $2 \mathrm{H}, \mathrm{CH}-\mathrm{CH}_{2}-\mathrm{C}_{\mathrm{Ar}}$ ).

\subsubsection{Synthesis of star RAFT agent pentaerythritoltetrakis-(3- (S-benzyltrithiocarbonyl)propionate) (star-RAFT-1)}<smiles>O=C(CCSC(=S)SCc1ccccc1)OCC(COC(=O)CCSC(=S)SCc1ccccc1)(COC(=O)CCSC(=S)SCc1ccccc1)COC(=O)CCSC(=S)SCc1ccccc1</smiles>

Pentaerythritol tetrakis(3-mercaptopropionate) $(1.00 \mathrm{~g}, 2.05 \mathrm{mmol}$, 1 eq.) was added to a suspension of potassium phosphate $(2.60 \mathrm{~g}$, $12.3 \mathrm{mmol}, 6 \mathrm{eq}$ ) in carbon disulfide ( $1.87 \mathrm{~g}, 12.3 \mathrm{mmol}, 6 \mathrm{eq}$.) under continuous stirring. After $30 \mathrm{~min}$ benzyl bromide $(2.10 \mathrm{~g}, 12.3 \mathrm{mmol}$, 6 eq.) was added and the stirring was continued for $24 \mathrm{~h}$. The reaction mixture was filtrated and the solvent was removed in vacuo. The crude product was purified by column chromatography using a mixture of $n$ hexane, ethyl acetate and triethylamine (1000:500:1) as eluent. The product was obtained as a yellow viscous oil $(0.260 \mathrm{~g}, 0.20 \mathrm{mmol}, 11 \%)$ 
${ }^{1} \mathrm{H}-N M R\left(300 \mathbf{M H z}, \mathbf{C}_{2} \mathbf{C l}_{\mathbf{4}} \mathbf{D}_{2}\right): \delta=7.41-7.23\left(\mathrm{~m}, 20 \mathrm{H},-\mathrm{CH}_{\mathrm{Ar}}\right), 4.62(\mathrm{~s}$, $\left.8 \mathrm{H},-\mathrm{SC}(=\mathrm{S}) \mathrm{S}-\mathrm{CH}_{2}-\mathrm{C}_{\mathrm{Ar}}\right), 4.16\left(\mathrm{~s}, 8 \mathrm{H},-\mathrm{C}\left(\mathrm{CH}_{2}\right)-\right), 3.62(\mathrm{t}, J=6.9 \mathrm{~Hz}, 8 \mathrm{H}$, $\left.\mathrm{CH}_{2}-\mathrm{CH}_{2}-\mathrm{SC}(=\mathrm{S}) \mathrm{S}-\right), 2.82\left(\mathrm{t}, J=6.9 \mathrm{~Hz}, 8 \mathrm{H}, \mathrm{CH}_{2}-\mathrm{CH}_{2}-\mathrm{SC}(=\mathrm{S}) \mathrm{S}-\right)$.

\subsubsection{Synthesis of RAFT agent S,S-Bis( $\alpha, \alpha^{\prime}$-dimethyl- $\alpha^{\prime \prime}$ - acetic acid)trithiocarbonate (DMATC)}<smiles>CC(C)(SC(=S)SC(C)(C)C(=O)O)C(=O)O</smiles>

A solution of carbon disulfide ( $27.4 \mathrm{~g}, 0.36 \mathrm{~mol}, 0.4 \mathrm{eq}$.$) , choloroform$ (107.5 g, $0.90 \mathrm{mmol}, 1 \mathrm{eq}$.$) acetone (52.3 g, 0.90 \mathrm{~mol}, 1 \mathrm{eq}$.$) ,$ tetrabutylammonium hydrogensulfate $(2.41 \mathrm{~g}, 7.10 \mathrm{mmol}$, catalytic amount) and toluene $(120 \mathrm{~mL})$ was cooled to $0^{\circ} \mathrm{C}$. A sodium hydroxide solution ( $50 \mathrm{wt} \%$ in water) was carefully added under permanent cooling to keep the temperature of the reaction mixture below $10{ }^{\circ} \mathrm{C}$. After completion of the addition the mixture was stirred for $24 \mathrm{~h}$. A precipitate was formed which was redissolved by the addition of water (900 $\mathrm{mL})$ and the aqueous phase was acidified with concentrated hydrochloric acid (120 mL). After 30 min of stirring the brown solid was filtrated and recrystallized from acetone to give the yellow crystalline product (12.5 g, $0.108 \mathrm{~mol}, 5 \%$ ).

${ }^{1} \mathrm{H}-\mathrm{NMR}$ (300 MHz, DMSO-d $)$ ): $\delta=12.89$ (s, $\left.2 \mathrm{H},-\mathrm{C}(=0) \mathrm{OH}\right), 1.60$ (s, $\left.12 \mathrm{H},-\mathrm{CH}_{3}\right)$.

\subsubsection{Synthesis of RAFT agent S,S-bis( $\alpha$-methyl- $\alpha$ - acetic acid)trithiocarbonate (MATC)}<smiles>CC(SC(=S)SC(C)C(=O)O)C(=O)O</smiles>

To a solution of potassium hydroxide $(6.60 \mathrm{~g}, 117.5 \mathrm{mmol}, 2.2 \mathrm{eq}$.) in water $(75 \mathrm{~mL})$, carbon disulfide ( $8.04 \mathrm{~g}, 105.6 \mathrm{mmol}, 2 \mathrm{eq}$.$) was added.$ Afterwards 2-bromopropionic acid $(8.075 \mathrm{~g}, 52.8 \mathrm{mmol}, 1 \mathrm{eq}$.) was dropwisely added and the solution was allowed to react for $72 \mathrm{~h}$ under continuous stirring. The solution was washed with methylene chloride $(5 \times 25 \mathrm{~mL})$. Then, the aqueous layer was acidified with hydrochloric acid and extracted with methylene chloride until the aqueous layer was no longer yellow. The organic layer was dried using magnesium sulfate and 
the solvent was removed in vacuo. The crude product was recrystallized from toluene and ethyl acetate (1:1) to yield the yellow product $(2.50 \mathrm{~g}$, mmol, $19 \%)$.

${ }^{1} \mathrm{H}-\mathrm{NMR}$ (300 MHz, DMSO-d $\mathbf{d}_{6}$ ): $\delta=13.26(\mathrm{~s}, 2 \mathrm{H},-\mathrm{C}(=0) 0 \mathrm{H}), 4.65$ (q, $J=7.3 \mathrm{~Hz}, 2 \mathrm{H},-\mathrm{CH}-), 1.52\left(\mathrm{~d}, J=7.3 \mathrm{~Hz}, 6 \mathrm{H},-\mathrm{CH}_{3}-\right.$ )

\subsubsection{Synthesis of polyfunctional RAFT agents poly-DMATC and poly-MATC}<smiles>CCCCCCCOC(=O)C(C)(C)SC(=S)SC(C)(C)C(C)=O</smiles>

poly-DMATC

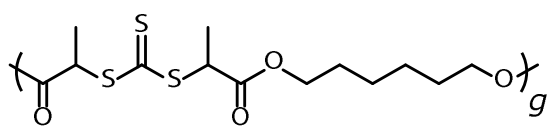

poly-MATC

The synthesis route is described for the preparation of poly-DMATC. Poly-MATC was obtained in a likewise procedure. DMATC $(6.00 \mathrm{~g}$, $21.2 \mathrm{mmol}, 1$ eq.), 1,6-hexanediol $(2.51 \mathrm{~g}, 21.2 \mathrm{mmol}, 1 \mathrm{eq}$.$) and p$ toluenesulfonic acid monohydrate $(0.89 \mathrm{~g}, 4.48 \mathrm{mmol}, 0.21 \mathrm{eq}$.) were suspended in cyclohexane $(4 \mathrm{~mL})$. The reaction mixture was heated under reflux at $120^{\circ} \mathrm{C}$ over $48 \mathrm{~h}$. After cooling the obtained brown solid was dissolved in methylene chloride $(10 \mathrm{~mL})$ and precipitated in excess amounts of cold methanol. The oligomeric RAFT agent was collected by centrifugation and decantation of the supernatant. It was then redissolved and the precipitation-centrifugation process was repeated twice. The product was dried in vacuo and characterized by SEC (see chapters 3 and 4).

For poly-MATC the respective weighed portions were: MATC $(2.01 \mathrm{~g}$, $7.91 \mathrm{mmol}, 1$ eq.), 1,6-hexanediol $(0.935 \mathrm{~g}, 7.91 \mathrm{mmol}, 1 \mathrm{eq}$.$) and$ $p$-toluenesulfonic acid monohydrate $(0.599 \mathrm{~g}, 3.10 \mathrm{mmol}, 0.4 \mathrm{eq}$.$) .$

\subsubsection{Synthesis of silica nanoparticles}

Ethanol (300 mL), methanol ( $400 \mathrm{~mL})$ and an ammonia solution in water $(33 \mathrm{wt} \%, 22.4 \mathrm{~mL})$ were placed in a flask ( $1 \mathrm{~L})$ and stirred for $5 \mathrm{~min}$. Tetraethyl orthosilicate $(25.2 \mathrm{~mL}, 113 \mathrm{mmol})$ was quickly added and stirring was continued for $20 \mathrm{~h}$. Subsequently, the solvent was removed under reduced pressure. The crude particles were washed with methanol $(3 \times 90 \mathrm{~mL})$, dispersed under ultrasonification and centrifugated to separate the particles from excessive solvent. Drying under vacuum yielded the particles a white powder $(7.10 \mathrm{~g}, 99 \%)$. 


\subsubsection{Synthesis of RAFT functionalized nanoparticles}

To a suspension of silica nanoparticles ( $3.00 \mathrm{~g}$ ) in 1,2-dimethoxyethane $(30 \mathrm{~mL})$ a solution of the RAFT agent BD2PT $(0.725 \mathrm{~g}, 2.10 \mathrm{mmol}, 1$ eq., see Figure $4-3)$ in 1,2-dimethoxyethane (10 mL) was dropwisely added under continuous stirring. Subsequently, a saturated solution of maleic anhydride in water $(0.2 \mathrm{~mL})$ was added and stirring was continued for $72 \mathrm{~h}$. Afterwards, the solvent was removed in vacuo. The RAFTimmobilized particles were purified by three cycles of washing with acetone $(30 \mathrm{~mL})$, centrifugation and subsequent redispersion. The product was obtained as a white powder $(2.98 \mathrm{~g}, 80 \%)$. The particle size distribution was characterized by DLS in toluene.

The RAFT agent was kindly provided by Dennis Hübner.

\subsubsection{Polymers}

\subsubsection{Homopolymerizations with APA}

The experiments refer to chapter 3, sections 4.2 and 4.3. In a typical experiment RAFT agent DMATC, poly-DMATC or star-RAFT-1, AIBN and APA were dissolved in 1,4-dioxane or methanol and the polymerization mixtures was degassed via three freeze-pump-thaw cycles. The polymerizations were conducted in a heated oil-bath at $60^{\circ} \mathrm{C}$ under argon atmosphere. Polymerizations were stoppd by exposing the reaction solutions to air and cooling with liquid nitrogen. All polymers were then precipitated in mixtures of $n$-hexane and ethyl acetate (1:1) and collected by centrifugation of the mixture and decantation of the supernatant. The polymers were redissolved with minimal amounts of 1,4-dioxane and then the precipitation-centrifugation process was repeated twice. The isolatd polymer was freeze-dried from 1,4-dioxane. The amounts of the used substances and polymerization conditions of the respective experiments are collated in Table 7-1.

\subsubsection{Copolymerizations of APA homopolymer with methyl acrylate}

The experiments refer to chapter 3 , sections 4.2 and 4.3. APA homopolymers were dissolved with AIBN and MA in methanol. The solutions were degassed via three freeze-pump-thaw cycles and the polymerizations were conducted in a heated oil-bath at $60^{\circ} \mathrm{C}$ under argon atmosphere. Polymerizations were stopped by exposing the reaction mixtures to air and cooling with liquid nitrogen. Afterwards, the 
solutions were poured into aluminum bowls and it was waited until remaining solvent and MA had evaporated. Polymers were then dried in a vacuum oven at $100{ }^{\circ} \mathrm{C}$ under reduced pressure. The amounts of the used substances and polymerization conditions of the respective experiments are collated in Table 7-2.

\subsubsection{Homopolymerization with methyl acrylate}

The experiment refers to chapter 3. In a typical experiment methyl acrylate $\left(c=3.48 \mathrm{~mol} \mathrm{~L}^{-1}\right)$, DMATC $\left(c=1.7410^{-2} \mathrm{~mol} \mathrm{~L}^{-1}\right)$, and AIBN $\left(c=4.3510^{-3} \mathrm{~mol} \mathrm{~L}^{-1}\right)$ in methanol were added to a flask equipped with a magnetic stirring bar and the mixture was degassed thoroughly via four freeze-pump-thaw cycles. The polymerization was conducted in a heated oil-bath at $60^{\circ} \mathrm{C}$ under argon atmosphere. The reaction mixture was poured into small aluminum bowls and the samples were dried in a vacuum oven at $100^{\circ} \mathrm{C}$ for $24 \mathrm{~h}$.

\subsubsection{Methylation of APA polymer samples}

In accordance with the work of Mori et al.,119 all APA-containing polymers had to be modified by methylation of the carboxylic acid groups prior to SEC characterization. Therefore, a modified literature procedure was applied ${ }^{254}$ using 3-methyl-1-( $p$-tolyl)triazene as methylating agent. $50 \mathrm{mg}$ of each polymer and $50 \mathrm{mg}$ of 3-methyl-1-( $p$ tolyl)triazene were dissolved in $4 \mathrm{~mL}$ of 1,4-dioxane and the mixture was stirred at $60^{\circ} \mathrm{C}$ for $72 \mathrm{~h}$. Thus it was ensured that an excess of methylating agent was added. Afterwards, $30 \mathrm{~mL}$ of dichloromethane was added to the reaction mixture, followed by work-up with hydrochloric acid $(2 \times 10 \mathrm{~mL})$. The organic phases were collected and the solvent was removed in vacuo, yielding samples that could be analyzed via SEC without further purification.

\subsubsection{Aminolysis of APA starpolymers}

The experiments refer to section 4.3. In a typical experiment $10 \mathrm{mg}$ of APA starpolymer was dissolved in $3 \mathrm{~mL}$ of hydrazine monohydrate. The solution was stirred for $1 \mathrm{~h}$ at room temperature and then the polymer was precipiated in hydrochloric acid. The precipitate was separated from the supernatant via centrifugation and the obtained polymer was subsequently investigated using SEC.228 


\subsubsection{Nanoparticle polymerizations}

Nanoparticle polymerizations were conducted as described in sections 7.3.8.1 and 7.3.8.3 but per gram of monomer, $50 \mathrm{mg}$ of RAFT functionalized nanoparticles were added to the polymerization mixture.

\subsubsection{Homopolymerizations with styrene}

Styrene polymerizations were conducted in bulk. In a typical polymerization procedure, solutions of RAFT agents and AIBN in styrene were weighed into polymerization vials and additional styrene was added with respect to the target degree of polymerization. The vials were degassed via bubbling with Argon for $15 \mathrm{~min}$ and consequently heated to $60{ }^{\circ} \mathrm{C}$ in a heating block under constant agitation. Polymerizations were stopped by cooling with liquid nitrogen and exposure to air. Polymers were dissolved in dichloromethane and precipitated in methanol three times to remove residual monomer and initiator. The amounts of the used substances and polymerization conditions of the respective experiments are collated in Table 7-3, Table 7-4 and Table 7-5.

\subsubsection{Copolymerizations of styrene homopolymer with $n$-butyl acrylate}

Copolymerizations with butyl acrylate were conducted with toluene as a solvent (volume fraction $=50 \%$ ). In a typical polymerization procedure, macro-RAFT agent $(300 \mathrm{mg})$ was weighed into a polymerization vial. AIBN $\left(c=1.610^{-3} \mathrm{~mol} \cdot \mathrm{L}^{-1}\right)$ dissolved in toluene, $n$-butyl acrylate $\left(c=4.40 \mathrm{~mol} \cdot \mathrm{L}^{-1}\right)$ and toluene $\left(c=6.17 \mathrm{~mol} \cdot \mathrm{L}^{-1}\right)$ were added. The vial was degassed via bubbling with Argon for 15 min and consequently heated to $60^{\circ} \mathrm{C}$ for $2.5 \mathrm{~h}$ in a heating block under constant agitation. Polymerization was stopped by cooling with liquid nitrogen and exposure to air. The polymer was dissolved in dichloromethane and precipitated in methanol three times to remove residual monomer and initiator.

\subsubsection{Cleavage by reaction with excess radicals}

Polymer samples were cleaved at the TTC groups with excess radicals according to a procedure previously reported. ${ }^{78}$ Typically, polymer $(10 \mathrm{mg})$ and AIBN $(10 \mathrm{mg}, 0.060 \mathrm{mmol})$ were dissolved in $1 \mathrm{~mL}$ of tetrahydrofuran in a polymerization vial and heated to $85{ }^{\circ} \mathrm{C}$ under 
constant agitation for $5 \mathrm{~h}$. Afterwards, the solvent was removed and the samples were directly characterized by SEC without further work-up.

Table 7-1 Polymerization conditions of APA homopolymerizations.

\begin{tabular}{|c|c|c|c|c|}
\hline RAFT agent & $\begin{array}{c}c_{\mathrm{APA}} / \\
\mathrm{mol} \mathrm{L}^{-1}\end{array}$ & $\begin{array}{l}c_{\mathrm{RAFT}} / \\
\mathrm{mol} \mathrm{L}^{-1}\end{array}$ & $\begin{array}{l}c_{\text {AIBN }} / \\
\mathrm{mol} \mathrm{L}^{-1}\end{array}$ & $t / \mathrm{h}$ \\
\hline DMATC & $9.1210^{-1}$ & $9.1210^{-2}$ & $2.2810^{-2}$ & 48 \\
\hline $\begin{array}{c}\text { poly-DMATC } \\
\text { (chapter } 3 \text { ) }\end{array}$ & $9.1210^{-1}$ & $1.0110^{-2}$ & $2.2710^{-2}$ & 48 \\
\hline $\begin{array}{l}\text { poly-DMATC } \\
\text { (section } 4.2 \text { ) }\end{array}$ & 1.37 & $6.9110^{-3}$ & $4.5710^{-3}$ & 12 \\
\hline star-RAFT- & 1.5 & $5.3210^{-2}$ & $2.1310^{-2}$ & 10 \\
\hline
\end{tabular}

Table 7-2 Polymerization conditions of copolymerizations with MA. Concentrations of macro-RAFT agents were calculated based on theoretical molar mass values.

\begin{tabular}{ccccc}
\hline \hline RAFT agent & $\begin{array}{c}c_{\mathrm{MA}} / \\
\mathrm{mol} \mathrm{L}^{-1}\end{array}$ & $\begin{array}{c}c_{\text {macro-RAFT }} / \\
\mathrm{mol} \mathrm{L}^{-1}\end{array}$ & $\begin{array}{c}c_{\mathrm{AIBN}} / \\
\mathrm{mol} \mathrm{L}^{-1}\end{array}$ & $t / \mathrm{h}$ \\
\hline $\begin{array}{c}\text { DMATC } \\
\text { poly-DMATC } \\
\text { (chapter 3) }\end{array}$ & 3.48 & $1.4110^{-3}$ & $3.5210^{-4}$ & 48 \\
$\begin{array}{c}\text { poly-DMATC } \\
\text { (section 4.2) }\end{array}$ & 3.48 & $1.5110^{-4}$ & $1.3610^{-4}$ & 48 \\
\begin{tabular}{l} 
star-RAFT- \\
\hline \hline
\end{tabular}
\end{tabular}


Table 7-3 Polymerization conditions of the mixing experiments with bi- and polyfunctional RAFT agents DMATC and poly-DMATC as shown in Table 4-15. For all samples a degree of polymerization of 300 monomer units per TTC-group was targeted. The AIBN concentration was $c(\mathrm{AIBN})=3.6 \mathrm{~mol} \cdot \mathrm{L}^{-1}$. The polymerization time was $17 \mathrm{~h}$ at $60^{\circ} \mathrm{C}$.

\begin{tabular}{ccc}
\hline \hline sample & $\begin{array}{c}c_{\text {DMATC }} / \\
\text { mol L }^{-1}\end{array}$ & $\begin{array}{c}c_{\text {poly-DMATC }} / \\
\text { mol L }^{-1}\end{array}$ \\
\hline 1 & $3.5410^{-2}$ & - \\
2 & $9.86 \cdot 10^{-3}$ & $1.95 \cdot 10^{-3}$ \\
3 & $4.58 \cdot 10^{-3}$ & $2.30 \cdot 10^{-3}$ \\
4 & $2.44 \cdot 10^{-3}$ & $2.45 \cdot 10^{-3}$ \\
5 & $1.29 \cdot 10^{-3}$ & $2.53 \cdot 10^{-3}$ \\
6 & $5.21 \cdot 10^{-4}$ & $2.60 \cdot 10^{-3}$ \\
7 & - & $2.62 \cdot 10^{-3}$ \\
\hline \hline
\end{tabular}

Table 7-4 Polymerization conditions of mixing experiments with star RAFT agent starRAFT-2 and polyfunctional RAFT agent poly-MATC as shown in Table 4-16. For all samples a degree of polymerization of 300 monomer units per TTC-group was targeted. The AIBN concentration was $c(\mathrm{AIBN})=3.6 \mathrm{~mol} \cdot \mathrm{L}^{-1}$. The polymerization time was $17 \mathrm{~h}$ at $60^{\circ} \mathrm{C}$.

\begin{tabular}{ccc}
\hline \hline sample & $\begin{array}{c}c_{\text {star-RAFT-2 }} / \\
\text { mol L }^{-1}\end{array}$ & $\begin{array}{c}c_{\text {poly-MATC }} / \\
\text { mol L }^{-1}\end{array}$ \\
\hline 1 & - & $3.41 \cdot 10^{-3}$ \\
2 & $3.3910^{-5}$ & $3.3910^{-3}$ \\
3 & $1.7510^{-4}$ & $3.2910^{-3}$ \\
4 & $3.5810^{-4}$ & $3.2310^{-3}$ \\
5 & $1.06 \cdot 10^{-3}$ & $2.97 \cdot 10^{-3}$ \\
6 & $2.53 \cdot 10^{-3}$ & $2.46 \cdot 10^{-3}$ \\
7 & $4.66 \cdot 10^{-3}$ & $1.58 \cdot 10^{-3}$ \\
8 & $2.0610^{-3}$ & - \\
\hline \hline
\end{tabular}


Table 7-5 Polymerization conditions of polystyrene homopolymers that were prepared via mixing of star RAFT agent star-RAFT-2 and polyfunctional RAFT agent poly-MATC as shown in Table 4-17. For all samples a degree of polymerization of 300 monomer units per TTC-group was targeted. The AIBN concentration was $c(\mathrm{AIBN})=$ $3.6 \mathrm{~mol} \cdot \mathrm{L}^{-1}$. The polymerization time was $17 \mathrm{~h}$ at $60^{\circ} \mathrm{C}$. The same weighed portions were used for all samples of star-5 and star- 10 .

\begin{tabular}{ccc}
\hline \hline sample & $\begin{array}{c}C_{\text {DMATC }} / \\
\text { mol L }^{-1}\end{array}$ & $\begin{array}{c}c_{\text {poly-DMATC }} / \\
\text { mol L }^{-1}\end{array}$ \\
\hline linear & - & $2.21 \cdot 10^{-3}$ \\
star-5 & $1.16 \cdot 10^{-3}$ & $2.21 \cdot 10^{-3}$ \\
star-7 & $1.57 \cdot 10^{-4}$ & $2.20 \cdot 10^{-3}$ \\
star-8 & $1.98 \cdot 10^{-4}$ & $2.20 \cdot 10^{-3}$ \\
star-10 & $2.45 \cdot 10^{-4}$ & $2.19 \cdot 10^{-3}$ \\
star-27 & $8.00 \cdot 10^{-4}$ & $2.18 \cdot 10^{-3}$ \\
star-75 & $2.26 \cdot 10^{-3}$ & $7.39 \cdot 10^{-4}$ \\
star-100 & $8.74 \cdot 10^{-3}$ & - \\
\hline \hline
\end{tabular}


Appendices 



\section{Appendix A}

\section{AFM images of biomimetic multiblock copolymers}

The AFM images shown below were recorded as part of the studies into biomimetic multiblock copolymers presented in section 3.2.2.
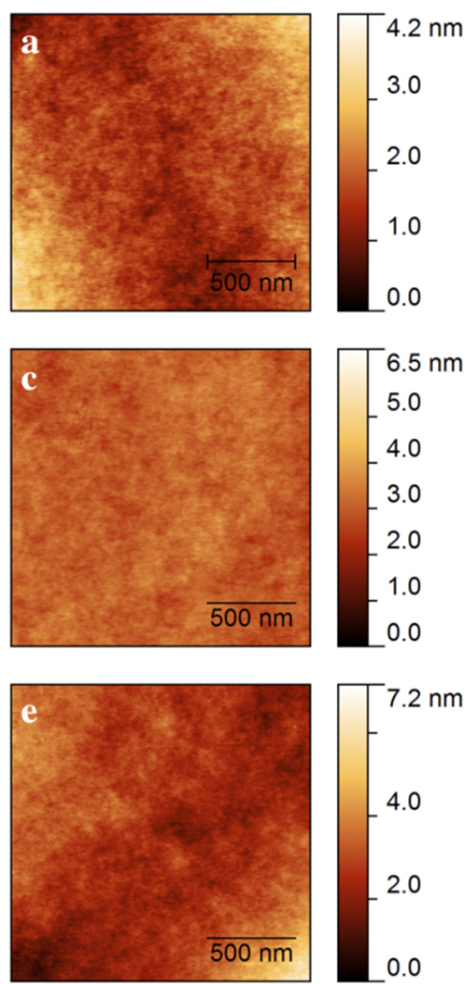
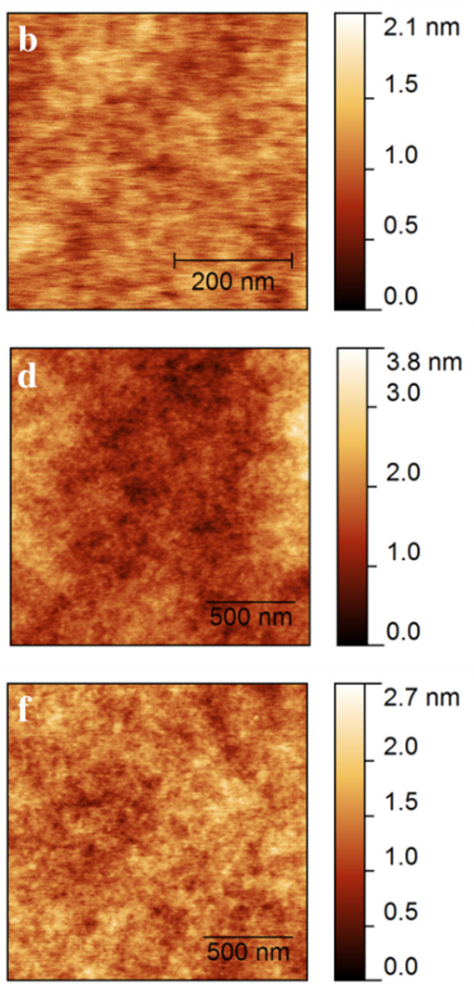

Figure A-1 AFM images of Tri-200 (a and b), Tri-250 (c), Tri-300 (d and e) and Multi200 (f). Images $a-d$ and $f$ were obtained after thermal annealing at $135^{\circ} \mathrm{C}$ for $24 \mathrm{~h}$. Image $e$ was obtained after solvent annealing under chloroform atmosphere for $24 \mathrm{~h}$. 


\section{Appendix B}

\section{Atomic Force Microscopy investigations of poly(styrene-block-n-butyl acrylate) copolymers}

It is known from literature, that star shaped block copolymers can be visualized using AFM. ${ }^{255}$ The Matyjaszewski group managed to visualize star block copolymers consisting of $n$-butyl acrylate and styrene, that were cast from dilute solutions, on a mica substrate. As the polar surface of the mica forces poly( $n$-butyl acrylate) segments to adopt highly stretched conformations, macromolecular topologies become visible. The authors prepared three arm star block copolymers with number average molar masses up to $170000 \mathrm{~g} \mathrm{~mol}^{-1}$. To match the conditions of the literature study, additional samples were prepared with higher number average molar masses (see Table A-1). As no specific comonomer ratio was targeted, the synthesized copolymers differ from the copolymer samples that were investigated via tensile testing (see Table 4-18). However, the relatively high content of $n$-butyl acrylate in the high molar mass copolymer STAR-10 $10^{\mathrm{HMW}}$ should facilitate visualization by AFM, because of the stretched conformations of the respective polymer segments.

Figure A-2 shows typical AFM images that were obtained for dilute solutions of STAR-10 ${ }^{\mathrm{HMW}}\left(1 \mu \mathrm{g} \mathrm{mL} \mathrm{m}^{-1}\right.$ in toluene). The surface does not exhibit any features that would indicate the presence of single molecular species. The Matyjaszewski group utilized solutions of 5 to $10 \mu \mathrm{g} \mathrm{mL}^{-1}$ in toluene, ${ }^{255}$ therefore, additional casting solutions were prepared with concentrations varying between 20 and $1 \mu \mathrm{g} \mathrm{mL}-1$. However, no effect on the recorded AFM images could be observed. It was concluded, that the visualization of single molecules is not possible for the prepared materials under the given conditions. Presumably, the discrimination of star species is hampered due to the larger amount of linear species in addition to large dispersity (see Table A-1). 
Table A-1 High molar mass copolymer samples that were prepared with poly-MATC and $10 \mathrm{~mol} \%$ of star-RAFT-2. SEC was measured versus linear polystyrene standards in THF. The comonomer ratio $r$ (BA:Styrene) was determined via NMR.

\begin{tabular}{lcccc}
\hline \hline \multicolumn{1}{c}{ sample } & $\begin{array}{c}\text { molar portion } \\
\text { of star-RAFT-2 }\end{array}$ & $\begin{array}{c}\bar{M}_{\mathrm{n}, \mathrm{SEC}} / \\
10^{5} \mathrm{~g} \mathrm{~mol}^{-1}\end{array}$ & $Ð$ & $r$ \\
\hline LINEAR $^{\mathrm{HMW}}$ & - & 3.0 & 1.87 & 0.90 \\
STAR-10 $^{\mathrm{HMW}}$ & $10 \%$ & 2.9 & 1.87 & 0.90 \\
\hline \hline
\end{tabular}

Consequently, AFM studies were continued using more concentrated casting solutions to examine an influence of the macromolecular topology on the microphase morphology. Therefore, copolymer samples LINEAR and STAR-10i were applied, as AFM investigations might aid in the interpretation of tensile testing results. Solutions $(0.5 \mathrm{wt} \%$ in toluene) were casted onto glass substrates. Representative AFM images are depicted in Figure A-3. For both copolymers, a flat surface was observed, indicating smooth coverage with polymer. This experimental finding is in accordance with the biomimetic multiblock copolymer systems (see section 3.2.2), that also did not exhibit any visible phase separation.
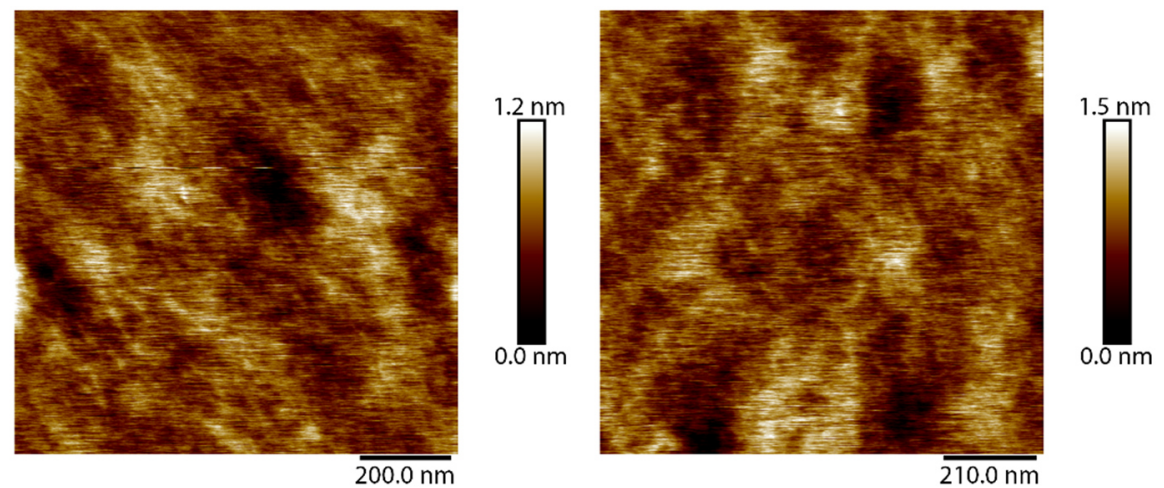

Figure A-2 Representative AFM images of STAR-10 $10^{\mathrm{HM}}$ on a mica substrate.

To increase the tendency of a copolymer to form microphase separated structures, the product $\chi N$ has to be increased, that is, larger average molar and hence average block length are required. Since the copolymer sample STAR-10 ${ }^{\mathrm{HMW}}$ exhibits significantly increased block lengths compared to STAR-10i, it was assumed that microphase separation could occur. Therefore, a more concentrated solution 
( $0.5 \mathrm{wt} \%$ in toluene) of STAR- $10^{\mathrm{HMW}}$ was applied in the casting process and the respective AFM images indeed show a microscopic structure (see Figure A-4 top). Interestingly, the observed spheres show a somewhat irregular arrangement, which is clearly visible at higher magnification (see Figure A-4 top, right). This observation is presumably a result of the larger dispersity. To validate the interpretation of phase separation, samples were investigated that were not thermally annealed (see Figure A-4 bottom). The AFM images show irregular arrangements of macromolecules or aggregates, proving that an ordering process must have occured after annealing. For comparison, a copolymer sample was prepared only with poly-MATC that exhibited comparable molar mass and composition to STAR-10 ${ }^{\mathrm{HMW}}$ (LINEAR ${ }^{\mathrm{HMW}}$, see Table A-1). The obtained AFM images also show spherical arrangements without any notable differences (see Figure A-5).

The latter experiments suggest, that minor addition of star RAFT agent (up to $10 \mathrm{~mol} \%$ ) to polyfunctional RAFT agent does not change the microscopic structure of thus prepared block copolymers. However, high molar masses of the block copolymers are required for the samples to exhibit visible microphase separation in AFM experiments. Hence, AFM investigations can not aid in the interpreation of results of the tensile testing experiments (see 4.4.4), as the prepared copolymers show significantly lower number average molar masses (see Table 4-18). The presented AFM results, nonetheless, offer various possibilities for future work. For example, high molar mass copolymers could be prepared with larger star-RAFT-2 ratios to attempt the visualization of single molecule star species via AFM. Yet, the presented work focused on a comprehensive examination of the influence of the mixing concept on the mechanical properties of copolymers and AFM studies could not be conducted in its entirety. 

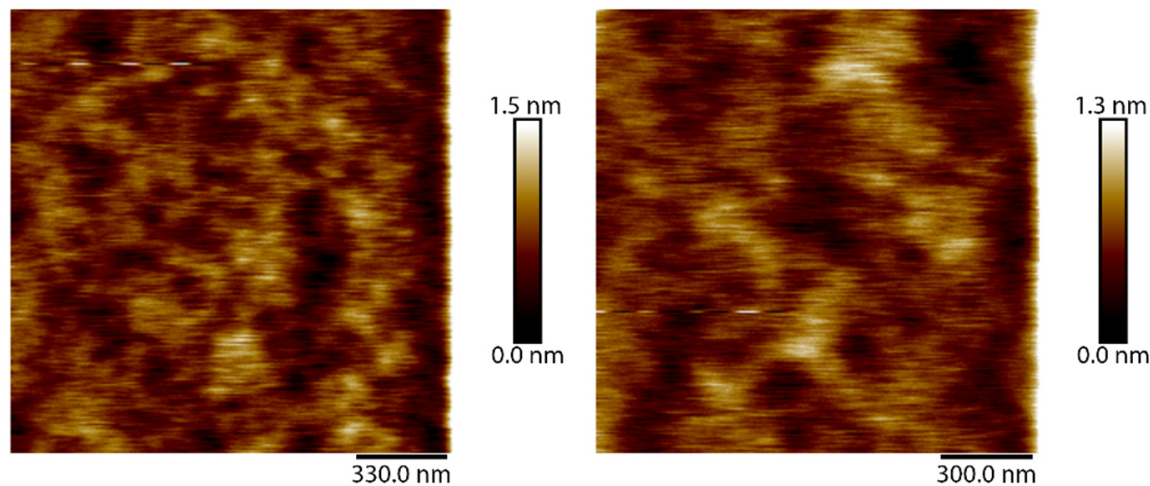

Figure A-3 Representative AFM images of LINEAR (left) and STAR-10i (right) on a glass substrate.
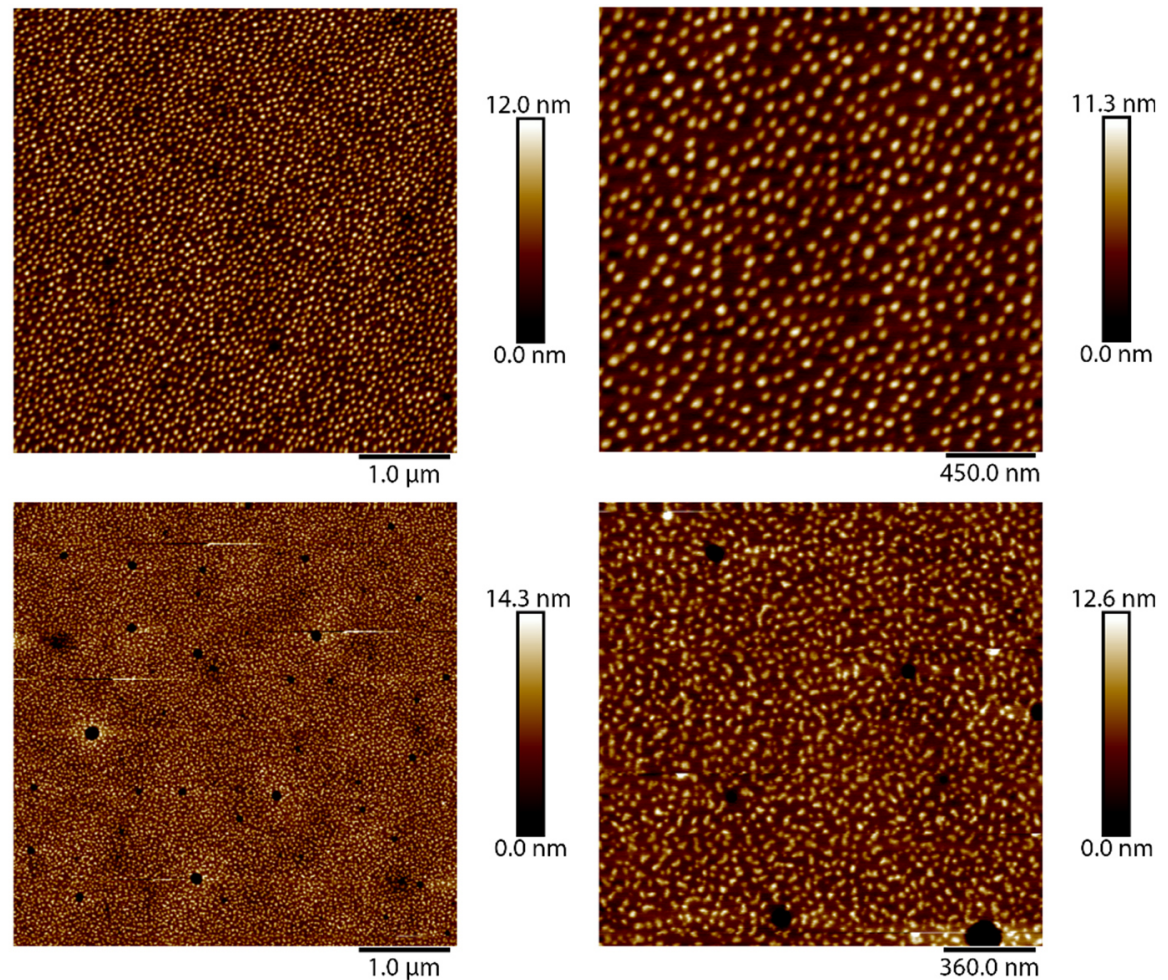

Figure A-4 Representative AFM images of STAR-10HMW on a glass substrate after overnight annealing at $100{ }^{\circ} \mathrm{C}$ under reduced pressure (top) and without annealing (bottom). 

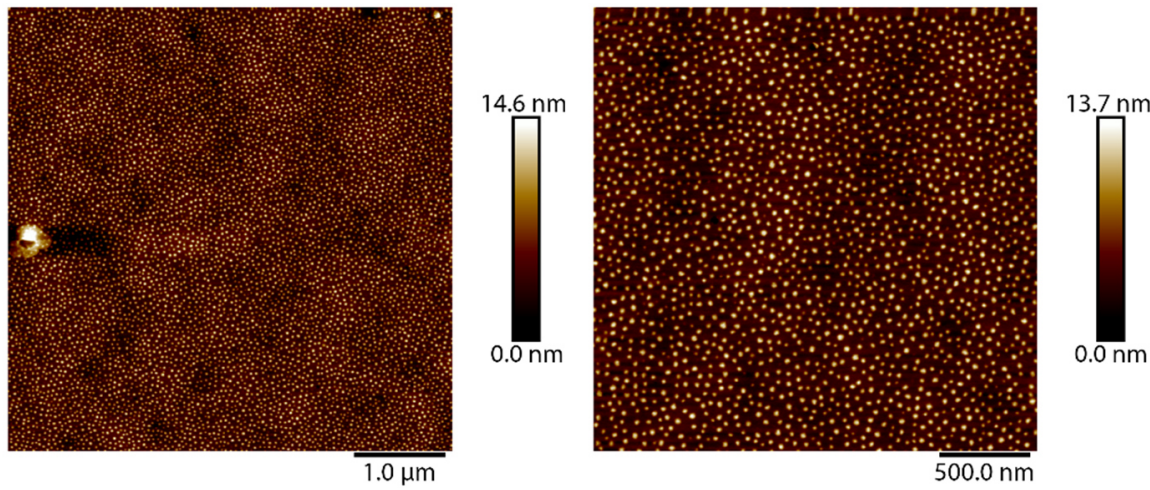

Figure A-5 Representative AFM images of LINEAR ${ }^{\mathrm{HMW}}$ on a glass substrate after overnight annealing at $100^{\circ} \mathrm{C}$ under reduced pressure. 


\section{Abbreviations and Acronyms}

$A_{0} \quad$ initial cross-section of a tensile specimen

$\AA \quad$ Ångström $\left(10^{-10} \mathrm{~m}\right)$

ABCN 1,1'-azobis(cyclohexanecarbonitrile)

AFM atomic force microscopy

AIBN 2,2'-Azobis(isobutyronitrile)

APA $\quad N$-acryloyl-L-phenylalanine

ATRP atom transfer radical polymerization

BA $n$-butyl acrylate

c concentration

${ }^{\circ} \mathrm{C} \quad$ degree Celsius

Đ dispersity $=\frac{\bar{M}_{\mathrm{w}}}{\bar{M}_{\mathrm{n}}}$

DLS dynamic light scattering

DMAc $\quad N, N$-dimethylacetamide

DMSO dimethyl sulfoxide

DP degree of polymerization

DSC differential scanning calorimetry

E Young's modulus

$\varepsilon \quad$ strain

$\varepsilon_{\text {br }} \quad$ strain at break

eq. equivalent 


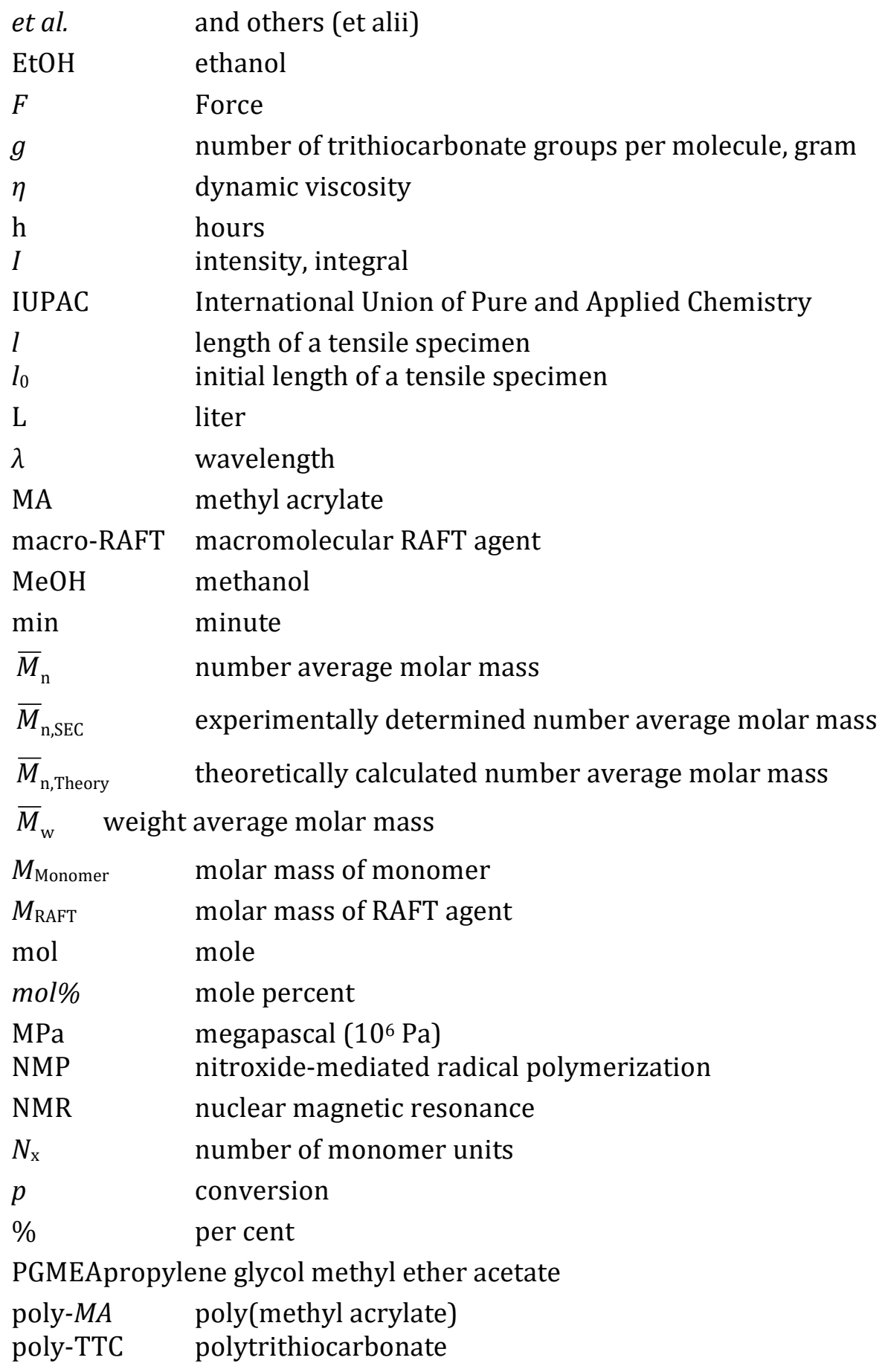

PGMEApropylene glycol methyl ether acetate poly-MA poly(methyl acrylate) poly-TTC polytrithiocarbonate 


$\begin{array}{ll}\text { ppm } & \text { parts per million } \\ r & \text { comonomer ratio } \\ \text { RAFT } & \text { reversible addition-fragmentation chain transfer } \\ \text { RDRP } & \text { reversible-deactivation radical polymerization } \\ R_{\mathrm{h}} & \text { hydrodynamic radius } \\ \text { RI } & \text { refractive index } \\ \text { S } & \text { second } \\ \text { SEC } & \text { size-exclusion chromatography } \\ \text { SNP } & \text { silica nanoparticle } \\ \sigma & \text { stress } \\ \sigma_{\text {UTS }} & \text { ultimate tensile strength } \\ \sigma_{\mathrm{y}} & \text { yield stress } \\ T & \text { temperature } \\ \text { TEM } & \text { transmission electron microscopy } \\ T_{\mathrm{g}} & \text { glass transition temperature } \\ \text { TGA } & \text { thermogravimetric analysis } \\ \text { THF } & \text { tetrahydrofuran } \\ \text { TIPS } & \text { triisopropylsilyl } \\ \text { TTC } & \text { trithiocarbonate } \\ U_{\mathrm{T}} & \text { toughness } \\ \text { UV } & \text { ultra violet } \\ \text { wt } \% & \text { weight percent } \\ x & \text { molar ratio } \\ \chi & \text { Flory-Huggins parameter }\end{array}$





\section{Bibliography}

(1) Bhushan, B. Phil. Trans. R. Soc. A 2009, 367, 1445-1486.

(2) Vincent, J. F. V.; Bogatyreva, O. A.; Bogatyrev, N. R.; Bowyer, A.; Pahl, A.-K. J. R. Soc. Interface 2006, 3, 471-482.

(3) Hardy, J. G.; Scheibel, T. R. Biochem. Soc. Trans. 2009, 37, 677-681.

(4) Keten, S.; Xu, Z.; Ihle, B.; Buehler, M. J. Nat. Mater. 2010, 9, 359367.

(5) Osaki, S. Nature 1996, 384, 419-419.

(6) Vollrath, F.; Knight, D. P. Nature 2001, 410, 541-548.

(7) Jelinski, L. W. Curr. Opin. Solid State Mater. Sci. 1998, 3, 237-245.

(8) Su, I.; Buehler, M. J. Nanotechnology 2016, 27, 302001.

(9) Rathore, O.; Sogah, D. Y. J. Am. Chem. Soc. 2001, 123, 5231-5239.

(10) Ebeling, B.; Eggers, M.; Vana, P. Macromolecules 2010, 43, 1028310290.

(11) Osaki, S. Polym. J. 2007, 39, 267-270.

(12) Osaki, S.; Yamamoto, K.; Matsuhira, T.; Sakai, H. Polym. J. 2016, 48, $1-5$.

(13) Lewis, R. V. Acc. Chem. Res. 1992, 25, 392-398.

(14) Osaki, S. Int. J. Biol. Macromol. 1999, 24, 283-287.

(15) Osaki, S.; Ishikawa, R. Polym. J. 2002, 34, 25-29.

(16) Liu, Y.; Shao, Z.; Vollrath, F. Nat. Mater. 2005, 4, 901-905.

(17) Rathore, O.; Sogah, D. Y. Macromolecules 2001, 34, 1477-1486.

(18) Simmons, A. H.; Michal, C. A.; Jelinski, L. W. Science 1996, 271, 8487.

(19) Sponner, A.; Vater, W.; Monajembashi, S.; Unger, E.; Grosse, F.; Weisshart, K. PLoS One 2007, 2, e998.

(20) Termonia, Y. Biomacromolecules 2004, 5, 2404-2407. 
(21) Vollrath, F.; Madsen, B.; Shao, Z. Proc. R. Soc. B Biol. Sci. 2001, 268, 2339-2346.

(22) Jin, H.-J.; Kaplan, D. L. Nature 2003, 424, 1057-1061.

(23) Eisoldt, L.; Smith, A.; Scheibel, T. Mater. Today 2011, 14, 80-86.

(24) Heim, M.; Keerl, D.; Scheibel, T. Angew. Chem. Int. Ed. 2009, 48, 3584-3596.

(25) Agnarsson, I.; Kuntner, M.; Blackledge, T. A. PLoS One 2010, 5, e11234.

(26) Porter, D.; Guan, J.; Vollrath, F. Adv. Mater. 2013, 25, 1275-1279.

(27) Savage, K. N.; Gosline, J. M. J. Exp. Biol. 2008, 211, 1937-1947.

(28) Hagn, F.; Thamm, C.; Scheibel, T.; Kessler, H. Angew. Chem. Int. Ed. 2011, 50, 310-313.

(29) Du, N.; Liu, X. Y.; Narayanan, J.; Li, L.; Lim, M. L. M.; Li, D. Biophys. J. 2006, 91, 4528-4535.

(30) Gao, H.; Ji, B.; Jager, I. L.; Arzt, E.; Fratzl, P. Proc. Natl. Acad. Sci. 2003, 100, 5597-5600.

(31) Giesa, T.; Pugno, N. M.; Wong, J. Y.; Kaplan, D. L.; Buehler, M. J. Adv. Mater. 2014, 26, 412-417.

(32) Meyers, M. A.; McKittrick, J.; Chen, P.-Y. Science 2013, 339, 773779.

(33) Zhou, C.; Leng, B.; Yao, J.; Qian, J.; Chen, X.; Zhou, P.; Knight, D. P.; Shao, Z. Biomacromolecules 2006, 7, 2415-2419.

(34) Johnson, J. C.; Wanasekara, N. D.; Korley, L. T. J. Biomacromolecules 2012, 13, 1279-1286.

(35) Shao, Z.; Vollrath, F.; Yang, Y.; Thøgersen, H. C. Macromolecules 2003, 36, 1157-1161.

(36) Omenetto, F. G.; Kaplan, D. L. Science 2010, 329, 528-531.

(37) Förster, N.; Schmidt, S.; Vana, P. Polymers 2015, 7, 695-716.

(38) Ren, J. M.; McKenzie, T. G.; Fu, Q.; Wong, E. H. H.; Xu, J.; An, Z.; Shanmugam, S.; Davis, T. P.; Boyer, C.; Qiao, G. G. Chem. Rev. 2016, 116, 6743-6836.

(39) Leclerc, E.; Daoud, M. Macromolecules 1997, 30, 293-300.

(40) Gersappe, D.; Harm, P. K.; Irvine, D.; Balazs, A. C. Macromolecules 1994, 27, 720-724.

(41) Eastwood, E.; Viswanathan, S.; O'Brien, C. P.; Kumar, D.; Dadmun, M. D. Polymer 2005, 46, 3957-3970. 
(42) Eastwood, E. A.; Dadmun, M. D. Macromolecules 2002, 35, 50695077.

(43) Seefried, C. G.; Koleske, J. V; Critchfield, F. E. J. Appl. Polym. Sci. 1975, 19, 2493-2502.

(44) Seefried, C. G.; Koleske, J. V; Critchfield, F. E. J. Appl. Polym. Sci. 1975, 19, 2503-2513.

(45) Bates, F. S. Science 1991, 251, 898-905.

(46) Lee, D.; Lee, S.-H.; Kim, S.; Char, K.; Park, J. H.; Bae, Y. H. J. Polym. Sci. Part B Polym. Phys. 2003, 41, 2365-2374.

(47) Garrett, J. T.; Siedlecki, C. A.; Runt, J. Macromolecules 2001, 34, 7066-7070.

(48) Koo, C. M.; Hillmyer, M. A.; Bates, F. S. Macromolecules 2006, 39, 667-677.

(49) Lee, I.; Panthani, T. R.; Bates, F. S. Macromolecules 2013, 46, 73877398.

(50) Ebeling, B.; Vana, P. Polymers 2011, 3, 719-739.

(51) Greene, A. C.; Zhu, J.; Pochan, D. J.; Jia, X.; Kiick, K. L. Macromolecules 2011, 44, 1942-1951.

(52) Wu, Y.; Wang, Q. I. J. Polym. Sci. Part A Polym. Chem. 2010, 48, 2425-2429.

(53) Sommerdijk, N. A. J. M.; Holder, S. J.; Hiorns, R. C.; Jones, R. G.; Nolte, R. J. M. Macromolecules 2000, 33, 8289-8294.

(54) Wu, C.; Xie, Z.; Zhang, G.; Zi, G.; Tu, Y.; Yang, Y.; Cai, P.; Nie, T. Chem. Commun. 2002, 2898-2899.

(55) Nunes, R. W.; Martin, J. R.; Johnson, J. F. Polym. Eng. Sci. 1982, 22, 205-228.

(56) Hadjiantoniou, N. A.; Triftaridou, A. I.; Kafouris, D.; Gradzielski, M.; Patrickios, C. S. Macromolecules 2009, 42, 5492-5498.

(57) Nagata, Y.; Masuda, J.; Noro, A.; Cho, D.; Takano, A.; Matsushita, Y. Macromolecules 2005, 38, 10220-10225.

(58) Kim, J. G.; Cowman, C. D.; LaPointe, A. M.; Wiesner, U.; Coates, G. W. Macromolecules 2011, 44, 1110-1113.

(59) Chiefari, J.; Chong, Y. K. (Bill); Ercole, F.; Krstina, J.; Jeffery, J.; Le, T. P. T.; Mayadunne, R. T. A.; Meijs, G. F.; Moad, C. L.; Moad, G.; Rizzardo, E.; Thang, S. H. Macromolecules 1998, 31, 5559-5562.

(60) Boyer, C.; Bulmus, V.; Davis, T. P.; Ladmiral, V.; Liu, J.; Perrier, S. 
Chem. Rev. 2009, 109, 5402-5436.

(61) Vana, P. Macromol. Symp. 2007, 248, 71-81.

(62) Moad, G.; Rizzardo, E.; Thang, S. H. Aust. J. Chem. 2012, 65, 9851076.

(63) Hill, M. R.; Carmean, R. N.; Sumerlin, B. S. Macromolecules 2015, 48, 5459-5469.

(64) Zhong, M.; Matyjaszewski, K. Macromolecules 2011, 44, 26682677.

(65) You, Y.-Z.; Hong, C.-Y.; Pan, C.-Y. Chem. Commun. 2002, 28002801.

(66) You, Y.-Z.; Hong, C.-Y.; Pan, C.-Y. Macromol. Rapid Commun. 2002, 23, 776-780.

(67) Motokucho, S.; Sudo, A.; Sanda, F.; Endo, T. Chem. Commun. 2002, 1946-1947.

(68) Bussels, R.; Bergman-Göttgens, C.; Meuldijk, J.; Koning, C. Macromolecules 2004, 37, 9299-9301.

(69) Bussels, R.; Koning, C. E. Tetrahedron 2005, 61, 1167-1174.

(70) Bussels, R.; Bergman-Göttgens, C.; Meuldijk, J.; Koning, C. Polymer 2005, 46, 8546-8554.

(71) Jia, Z.; Xu, X.; Fu, Q.; Huang, J. J. Polym. Sci. Part A Polym. Chem. 2006, 44, 6071-6082.

(72) Pavlović, D.; Linhardt, J. G.; Künzler, J. F.; Shipp, D. A. J. Polym. Sci. Part A Polym. Chem. 2008, 46, 7033-7048.

(73) Yan, J.-J.; Wang, Z.-K.; Lin, X.-S.; Hong, C.-Y.; Liang, H.-J.; Pan, C.-Y.; You, Y.-Z. Adv. Mater. 2012, 24, 5617-5624.

(74) Roy, A.-L.; Bui, C.; Rau, I.; Kajzar, F.; Charleux, B.; Save, M.; Kreher, D.; Attias, A. Polymer 2014, 55, 782-787.

(75) Duwez, A.-S.; Guillet, P.; Colard, C.; Gohy, J.-F.; Fustin, C.-A. Macromolecules 2006, 39, 2729-2731.

(76) Rossner, C.; Ebeling, B.; Vana, P. ACS Macro Lett. 2013, 2, 10731076.

(77) Rossner, C.; Glatter, O.; Saldanha, O.; Vana, P. Langmuir 2015, 31, 10573-10582.

(78) Ebeling, B.; Vana, P. Macromolecules 2013, 46, 4862-4871.

(79) Rossner, C.; Vana, P. Angew. Chem. Int. Ed. 2014, 53, 1263912642. 
(80) Berek, D. J. Sep. Sci. 2010, 33, 315-335.

(81) Liu, Y.; Cavicchi, K. A. Macromol. Chem. Phys. 2009, 210, 16471653.

(82) Flory, P. J.J. Am. Chem. Soc. 1936, 58, 1877-1885.

(83) Schwartzl, F. R. Polymermechanik - Struktur und mechanisches Verhalten von Polmeren; Berlin, 1990.

(84) Martin, J. R.; Johnson, J. F.; Cooper, A. R. J. Macromol. Sci. Part C 1972, 8, 57-199.

(85) Bi, L.-K.; Fetters, L. J. Macromolecules 1976, 9, 732-742.

(86) Kushner, A. M.; Guan, Z. Angew. Chem. Int. Ed. 2011, 50, 90269057.

(87) Smith, B. L.; Schäffer, T. E.; Viani, M.; Thompson, J. B.; Frederick, N. A.; Kindt, J.; Belcher, A.; Stucky, G. D.; Morse, D. E.; Hansma, P. K. Nature 1999, 399, 761-763.

(88) van Hest, J. C. M.; Tirrell, D. A. Chem. Commun. 2001, 1897-1904.

(89) Shao, Z.; Vollrath, F. Nature 2002, 418, 741-741.

(90) Xu, M.; Lewis, R. V. Proc. Natl. Acad. Sci. USA 1990, 87, 7120-7124.

(91) Porter, D.; Vollrath, F. Nano Today 2007, 2, 6.

(92) Studart, A. R. Adv. Mater. 2012, 24, 5024-5044.

(93) Lazaris, A.; Arcidiacono, S.; Huang, Y.; Zhou, J.-F.; Duguay, F.; Chretien, N.; Welsh, E. A.; Soares, J. W.; Karatzas, C. N. Science 2002, 295, 472-476.

(94) Elices, M.; Guinea, G. V.; Plaza, G. R.; Karatzas, C.; Riekel, C.; AgullóRueda, F.; Daza, R.; Pérez-Rigueiro, J. Macromolecules 2011, 44, 1166-1176.

(95) Yao, J.; Xiao, D.; Chen, X.; Zhou, P.; Yu, T.; Shao, Z. Macromolecules 2003, 36, 7508-7512.

(96) Liu, H.; Xu, W.; Zhao, S.; Huang, J.; Yang, H.; Wang, Y.; Ouyang, C. J. Appl. Polym. Sci. 2010, 117, 235-242.

(97) Liu, H.; Zhou, J.; Liu, X.; Zuo, D.; Gu, S.; Xu, W. J. Appl. Polym. Sci. 2013, 130, 631-637.

(98) Liu, H.; Zhou, J.; Zuo, D.; Liu, X.; Gu, S.; Xu, W. J. Appl. Polym. Sci. 2014, 131, 1-6.

(99) Guan, Z. Polym. Int. 2007, 56, 467-473.

(100) Huang, H.; Hu, J.; Zhu, Y. Macromol. Biosci. 2013, 13, 161-166.

(101) Yamauchi, K.; Lizotte, J. R.; Long, T. E. Macromolecules 2003, 36, 
1083-1088.

(102) Ajji, Z. Rev. Roum. Chim. 2008, 53, 1065-1068.

(103) Tokareva, O.; Jacobsen, M.; Buehler, M.; Wong, J.; Kaplan, D. L. Acta Biomater. 2014, 10, 1612-1626.

(104) Ebrahimi, D.; Tokareva, O.; Rim, N. G.; Wong, J. Y.; Kaplan, D. L.; Buehler, M. J. ACS Biomater. Sci. Eng. 2015, 1, 864-876.

(105) Biemond, G. J. E.; Feijen, J.; Gaymans, R. J. Macromol. Mater. Eng. 2009, 294, 492-501.

(106) Cui, J.; del Campo, A. Chem. Commun. 2012, 48, 9302-9304.

(107) Kuo, S.-W.; Tsai, H.-T. J. Appl. Polym. Sci. 2011, 123, 3275-3282.

(108) Elkins, C. L.; Park, T.; McKee, M. G.; Long, T. E. J. Polym. Sci. Part A Polym. Chem. 2005, 43, 4618-4631.

(109) Cui, J.; Wang, D.; Koynov, K.; Del Campo, A. ChemPhysChem 2013, 14, 2932-2938.

(110) ten Cate, T. A.; Sijbesma, R. P. Macromol. Rapid Commun. 2002, 23, 1094-1112.

(111) Kautz, H.; van Beek, D. J. M.; Sijbesma, R. P.; Meijer, E. W. Macromolecules 2006, 39, 4265-4267.

(112) Feldman, K. E.; Kade, M. J.; de Greef, T. F. A.; Meijer, E. W.; Kramer, E. J.; Hawker, C. J. Macromolecules 2008, 41, 4694-4700.

(113) Aida, T.; Meijer, E. W.; Stupp, S. I. Science 2012, 335, 813-817.

(114) Bosman, A. W.; Sijbesma, R. P.; Meijer, E. W. Mater. Today 2004, 34-39.

(115) Beijer, F. H.; Sijbesma, R. P.; Kooijman, H.; Spek, A. L.; Meijer, E. W. J. Am. Chem. Soc. 1998, 120, 6761-6769.

(116) Beijer, F. H.; Kooijman, H.; Spek, A. L.; Sijbesma, R. P.; Meijer, E. W. Angew. Chem. 1998, 110, 79-82.

(117) Söntjens, S. H. M.; Sijbesma, R. P.; van Genderen, M. H. P.; Meijer, E. W. J. Am. Chem. Soc. 2000, 122, 7487-7493.

(118) Sun, H.; Lee, H. H.; Blakey, I.; Dargaville, B.; Chirila, T. V.; Whittaker, A. K.; Smith, S. C. J. Phys. Chem. B 2011, 115, 11053-11062.

(119) Mori, H.; Matsuyama, M.; Sutoh, K.; Endo, T. Macromolecules 2006, 39, 4351-4360.

(120) Mori, H.; Endo, T. Macromol. Rapid Commun. 2012, 33, 10901107.

(121) El-Newehy, M. H.; Elsherbiny, A. S.; Mori, H. J. Appl. Polym. Sci. 
2013, 127, 4918-4926.

(122) Du, J.; O'Reilly, R. K. Macromol. Chem. Phys. 2010, 211, 1530-1537.

(123) Mori, H.; Matsuyama, M.; Endo, T. Macromol. Chem. Phys. 2008, 209, 2100-2112.

(124) Mori, H.; Matsuyama, M.; Endo, T. Macromol. Chem. Phys. 2009, 210, 217-229.

(125) Spontak, R. J.; Smith, S. D. J. Polym. Sci. Part B Polym. Phys. 2001, 39, 947-955.

(126) Hendrich, M. Synthese und Eigenschaften von biomimetischen Multiblock-Copolymeren, Georg-August-Universität Göttingen, 2013.

(127) Chong, Y. K.; Le, T. P. T.; Moad, G.; Rizzardo, E.; Thang, S. H. Macromolecules 1999, 32, 2071-2074.

(128) Qiao, L.; Leibig, C.; Hahn, S. F.; Winey, K. I. Ind. Eng. Chem. Res. 2006, 45, 5598-5602.

(129) Barner-Kowollik, C.; Heuts, J. P. A.; Davis, T. P. J. Polym. Sci. Part A Polym. Chem. 2001, 39, 656-664.

(130) Boschmann, D.; Mänz, M.; Pöppler, A.-C.; Sörensen, N.; Vana, P. J. Polym. Sci. Part A Polym. Chem. 2008, 46, 7280-7286.

(131) Schwabe, M.; Rotzoll, R.; Küchemann, S.; Nadimpalli, K.; Vana, P.; Samwer, K. Macromol. Chem. Phys. 2010, 211, 1673-1677.

(132) Hentschel, J.; Kushner, A. M.; Ziller, J.; Guan, Z. Angew. Chem. 2012, 124, 10713-10717.

(133) Puskas, J. E.; Antony, P.; El Fray, M.; Altstädt, V. Eur. Polym. J. 2003, 39, 2041-2049.

(134) Bates, F. S.; Fredrickson, G. H. Annu. Rev. Phys. Chem. 1990, 41, 525-557.

(135) Matsen, M. W.; Thompson, R. B. J. Chem. Phys. 1999, 111, 71397146.

(136) Wu, P. D.; Van Der Giessen, E. J. Mech. Phys. Solids 1993, 41, 427456.

(137) Fu, B. X.; Hsiao, B. S.; Pagola, S.; Stephens, P.; White, H.; Rafailovich, M.; Sokolov, J.; Mather, P. T.; Jeon, H. G.; Phillips, S.; Lichtenhan, J.; Schwab, J. Polymer 2001, 42, 599-611.

(138) Meijer, H. E. H.; Govaert, L. E. Prog. Polym. Sci. 2005, 30, 915-938.

(139) Aharoni, S. M. Macromolecules 1983, 16, 1722-1728. 
(140) Koo, C. M.; Wu, L.; Lim, L. S.; Mahanthappa, M. K.; Hillmyer, M. A.; Bates, F. S. Macromolecules 2005, 38, 6090-6098.

(141) Nathani, H.; Dasari, A.; Misra, R. D. K. Acta Mater. 2004, 52, 32173227.

(142) Kambour, R. P. Polym. Eng. Sci. 1968, 8, 281-289.

(143) Kambour, R. P. J. Polym. Sci. Macromol. Rev. 1973, 7, 1-154.

(144) Dasari, A.; Rohrmann, J.; Misra, R. D. K. Mater. Sci. Eng. 2003, A351, 200-213.

(145) Breuer, H.; Haaf, F.; Stabenow, J. J. Macromol. Sci. Part B Phys. 1977, 14, 387-417.

(146) Liu, Y.; Kennard, C. H. L.; Truss, R. W.; Calos, N. J. Polymer 1997, 38, 2797-2805.

(147) Liu, Y. A. N.; Truss, R. W. J. Polym. Sci. Part B Polym. Phys. 1994, 2037-2047.

(148) Kushner, A. M.; Vossler, J. D.; Williams, G. A.; Guan, Z. J. Am. Chem. Soc. 2009, 131, 8766-8768.

(149) Liu, C.; Qin, H.; Mather, P. T. J. Mater. Chem. 2007, 17, 1543-1558.

(150) Schuetz, J.-H.; Peng, W.; Vana, P. Polym. Chem. 2015, 6, 1714-1726.

(151) Cordier, P.; Tournilhac, F.; Soulié-Ziakovic, C.; Leibler, L. Nature 2008, 451, 977-980.

(152) Blaiszik, B. J.; Kramer, S. L. B.; Olugebefola, S. C.; Moore, J. S.; Sottos, N. R.; White, S. R. Annu. Rev. Mater. Res. 2010, 40, 179-211.

(153) Chen, Y.; Kushner, A. M.; Williams, G. A.; Guan, Z. Nat. Chem. 2012, 4, 467-472.

(154) Jud, K.; Kausch, H. H. Polym. Bull. 1979, 1, 697-707.

(155) Wu, D. Y.; Meure, S.; Solomon, D. Prog. Polym. Sci. 2008, 33, 479522.

(156) Murthy, N. S.; Bray, R. G.; Correale, S. T.; Moore, R. A. F. Polymer 1995, 36, 3863-3873.

(157) Ajji, A.; Guèvremont, J.; Cole, K. C.; Dumoulin, M. M. Polymer 1996, 37, 3707-3714.

(158) Araki, Y.; Shimizu, D.; Hori, Y.; Nakatani, K.; Saito, H. Polym. J. 2013, $45,1140-1145$.

(159) Luo, Y.; Wang, X.; Zhu, Y.; Li, B.-G.; Zhu, S. Macromolecules 2010, $43,7472-7481$.

(160) Fei, P.; Cavicchi, K. A. ACS Appl. Mater. Interfaces 2010, 2, 2797- 
2803.

(161) Zhao, Y.; Ning, N.; Hu, X.; Li, Y.; Chen, F.; Fu, Q. Polymer 2012, 53, 4310-4317.

(162) Fernández-d'Arlas, B.; Ramos, J. A.; Saralegi, A.; Corcuera, M.; Mondragon, I.; Eceiza, A. Macromolecules 2012, 45, 3436-3443.

(163) Ruoppolo, M.; Vinci, F.; Klink, T. A.; Raines, R. T.; Marino, G. Biochemistry 2000, 39, 12033-12042.

(164) Mooibroek, H.; Cornish, K. Appl. Microbiol. Biotechnol. 2000, 53, 355-365.

(165) Agrawal, A.; Konno, K. Annu. Rev. Ecol. Evol. Syst. 2009, 40, 311331.

(166) Ozbas, B.; Toki, S.; Hsiao, B. S.; Chu, B.; Register, R. A.; Aksay, I. A.; Prud'homme, R. K.; Adamson, D. H. J. Polym. Sci. Part B Polym. Phys. 2012, 50, 718-723.

(167) Ahrends, A.; Hollingsworth, P. M.; Ziegler, A. D.; Fox, J. M.; Chen, H.; $\mathrm{Su}, \mathrm{Y}$.; Xu, J. Glob. Environ. Chang. 2015, 34, 48-58.

(168) Fakirov, S.; Gogeva, T. Makromol. Chemie 1990, 191, 615-624.

(169) Fakirov, S.; Gogeva, T. Makromol. Chemie 1990, 191, 603-614.

(170) Jenekhe, S. A.; Chen, X. L. Science 1998, 279, 1903-1907.

(171) Hart, K. E.; Abbott, L. J.; Lísal, M.; Colina, C. M. J. Chem. Phys. 2014, 141, 204902.

(172) Shim, J. S.; Kennedy, J. P. J. Polym. Sci. Part A Polym. Chem. 1999, 37, 815-824.

(173) Weidisch, R.; Laatsch, J.; Michler, G. H.; Arnold, M.; Schade, B.; Fischer, H. Macromolecules 2002, 35, 6585-6591.

(174) Kumar, S. K.; Krishnamoorti, R. Annu. Rev. Chem. Biomol. Eng. 2010, 1, 37-58.

(175) Akcora, P.; Liu, H.; Kumar, S. K.; Moll, J.; Li, Y.; Benicewicz, B. C.; Schadler, L. S.; Acehan, D.; Panagiotopoulos, A. Z.; Pryamitsyn, V.; Ganesan, V.; Ilavsky, J.; Thiyagarajan, P.; Colby, R. H.; Douglas, J. F. Nat. Mater. 2009, 8, 354-359.

(176) Fu, S.-Y.; Feng, X.-Q.; Lauke, B.; Mai, Y.-W. Composites: Part B 2008, 39, 933-961.

(177) Zou, H.; Wu, S.; Shen, J. Chem. Rev. 2008, 108, 3893-3957.

(178) Crosby, A. J.; Lee, J.-Y. Polym. Rev. 2007, 47, 217-219.

(179) Nan, C.-W.; Shen, Y.; Ma, J. Annu. Rev. Mater. Res. 2010, 40, 131- 
151.

(180) Akcora, P.; Kumar, S. K.; Moll, J.; Lewis, S.; Schadler, L. S.; Li, Y.; Benicewicz, B. C.; Sandy, A.; Narayanan, S.; Ilavsky, J.; Thiyagarajan, P.; Colby, R. H.; Douglas, J. F. Macromolecules 2010, 43, 1003-1010.

(181) Moll, J. F.; Akcora, P.; Rungta, A.; Gong, S.; Colby, R. H.; Benicewicz, B. C.; Kumar, S. K. Macromolecules 2011, 44, 7473-7477.

(182) Hashemi, A.; Jouault, N.; Williams, G. A.; Zhao, D.; Cheng, K. J.; Kysar, J. W.; Guan, Z.; Kumar, S. K. Nano Lett. 2015, 15, 5465-5471.

(183) Natarajan, B.; Neely, T.; Rungta, A.; Benicewicz, B. C.; Schadler, L. S. Macromolecules 2013, 46, 4909-4918.

(184) Maillard, D.; Kumar, S. K.; Fragneaud, B.; Kysar, J. W.; Rungta, A.; Benicewicz, B. C.; Deng, H.; Brinson, L. C.; Douglas, J. F. Nano Lett. 2012, 12, 3909-3914.

(185) Kumar, S. K.; Jouault, N.; Benicewicz, B.; Neely, T. Macromolecules 2013, 46, 3199-3214.

(186) Chen, Q.; Gong, S.; Moll, J.; Zhao, D.; Kumar, S. K.; Colby, R. H. ACS Macro Lett. 2015, 4, 398-402.

(187) Balazs, A. C.; Emrick, T.; Russell, T. P. Science 2006, 314, 11071110.

(188) Trask, R. S.; Williams, H. R.; Bond, I. P. Bioinspir. Biomim. 2007, 2, P1-P9.

(189) Petrovic, Z. S.; Javni, I.; Waddon, A.; Bánhegyi, G. J. Appl. Polym. Sci. 2000, 76, 133-151.

(190) Cho, J.; Joshi, M. S.; Sun, C. T. Compos. Sci. Technol. 2006, 66, 19411952.

(191) Bockstaller, M. R.; Mickiewicz, R. A.; Thomas, E. L. Adv. Mater. 2005, 17, 1331-1349.

(192) Sugimoto, H.; Daimatsu, K.; Nakanishi, E.; Ogasawara, Y.; Yasumura, T.; Inomata, K. Polymer 2006, 47, 3754-3759.

(193) Li, C.; Benicewicz, B. C. Macromolecules 2005, 38, 5929-5936.

(194) Edgecombe, S. R.; Gardiner, J. M.; Matsen, M. W. Macromolecules 2002, 35, 6475-6477.

(195) Rungta, A.; Natarajan, B.; Neely, T.; Dukes, D.; Schadler, L. S.; Benicewicz, B. C. Macromolecules 2012, 45, 9303-9311.

(196) Zhao, D.; Di Nicola, M.; Khani, M. M.; Jestin, J.; Benicewicz, B. C.; Kumar, S. K. ACS Macro Lett. 2016, 5, 790-795. 
(197) Zhao, D.; Di Nicola, M.; Khani, M. M.; Jestin, J.; Benicewicz, B. C.; Kumar, S. K. Soft Matter 2016, 12, 7241-7247.

(198) Heng, C.; Liu, M.; Wang, K.; Deng, F.; Huang, H.; Wan, Q.; Hui, J.; Zhang, X.; Wei, Y. Ceram. Int. 2015, 41, 15075-15082.

(199) Stöber, W.; Fink, A.; Bohn, E. J. Colloid Interface Sci. 1968, 26, 6269.

(200) Wang, X.-D.; Shen, Z.-X.; Sang, T.; Cheng, X.-B.; Li, M.-F.; Chen, L.-Y.; Wang, Z.-S. J. Colloid Interface Sci. 2010, 341, 23-29.

(201) Ebeling, B.; Ehlers, F.; Vana, P. Nachrichten aus der Chemie 2014, $62,24-28$.

(202) Semsarilar, M.; Perrier, S. Nat. Chem. 2010, 2, 811-820.

(203) Perrier, S.; Takolpuckdee, P.; Mars, C. A. Macromolecules 2005, 38, 6770-6774.

(204) Rowe, M. D.; Hammer, B. A. G.; Boyes, S. G. Macromolecules 2008, 41, 4147-4157.

(205) Dietrich, M.; Glassner, M.; Gruendling, T.; Schmid, C.; Falkenhagen, J.; Barner-Kowollik, C. Polym. Chem. 2010, 1, 634.

(206) Schmitz, K. S. An Introduction to Dynamic Light Scattering by Macromolecules; Academic Press, 1990.

(207) Papakonstantopoulos, G. J.; Yoshimoto, K.; Doxastakis, M.; Nealey, P. F.; de Pablo, J. J. Phys. Rev. E 2005, 72, 31801.

(208) Yang, F.; Ou, Y.; Yu, Z. J. Appl. Polym. Sci. 1998, 69, 355-361.

(209) Shang, X. Y.; Zhu, Z. K.; Yin, J.; Ma, X. D. Chem. Mater. 2002, 14, 7177.

(210) Finnigan, B.; Jack, K.; Campbell, K.; Halley, P.; Truss, R.; Casey, P.; Cookson, D.; King, S.; Martin, D. Macromolecules 2005, 38, 73867396.

(211) Spruijt, E.; Biesheuvel, P. M.; de Vos, W. M. Phys. Rev. E 2015, 91, 12601.

(212) Peng, C.-C.; Göpfert, A.; Drechsler, M.; Abetz, V. Polym. Adv. Technol. 2005, 16, 770-782.

(213) WetterKontor. Höchst- und Tiefstwerte in Deutschland am 15.07.2015

www.wetterkontor.de/de/deutschland_extremwerte.asp?id=20 150715.

(214) Lai, S.-M.; Liu, S.-D. Polym. Eng. Sci. 2007, 47, 77-86. 
(215) Laaksonen, P.; Walther, A.; Malho, J.; Kainlauri, M.; Ikkala, O.; Linder, M. B. Angew. Chem. Int. Ed. 2011, 50, 8688-8691.

(216) Kim, H.-W.; Song, J.-H.; Kim, H.-E. Adv. Funct. Mater. 2005, 15, 1988-1994.

(217) Ciprai, D.; Jacob, K.; Tannenbaum, R. Macromolecules 2006, 39, 6565-6573.

(218) Maiti, P.; Nam, P. H.; Okamoto, M.; Hasegawa, N.; Usuki, A. Macromolecules 2002, 35, 2042-2049.

(219) Sarkar, B.; Alexandridis, P. Prog. Polym. Sci. 2015, 40, 33-62.

(220) Skey, J.; Willcock, H.; Lammens, M.; Du Prez, F.; O’Reilly, R. K. Macromolecules 2010, 43, 5949-5955.

(221) Bi, L.-K.; Fetters, L. J. Macromolecules 1975, 8 (1), 90-92.

(222) Olvera de la Cruz, M.; Sanchez, I. C. Macromolecules 1986, 19, 2501-2508.

(223) Boschmann, D.; Vana, P. Polym. Bull. 2005, 53, 231-242.

(224) Barner-Kowollik, C.; Davis, T. P.; Stenzel, M. H. Aust. J. Chem. 2006, 59, 719-727.

(225) Barner, L.; Davis, T. P.; Stenzel, M. H.; Barner-Kowollik, C. Macromol. Rapid Commun. 2007, 28, 539-559.

(226) Boschmann, D.; Edam, R.; Schoenmakers, P. J.; Vana, P. Polymer 2008, 49, 5199-5208.

(227) Boschmann, D.; Vana, P. Macromolecules 2007, 40, 2683-2693.

(228) Shen, W.; Qiu, Q.; Wang, Y.; Miao, M.; Li, B.; Zhang, T.; Cao, A.; An, Z. Macromol. Rapid Commun. 2010, 31, 1444-1448.

(229) Adhikari, R.; Lach, R.; Michler, G. H.; Weidisch, R.; Grellmann, W.; Knoll, K. Polymer 2002, 43, 1943-1947.

(230) Adhikari, R.; Michler, G. H.; Goerlitz, S.; Knoll, K. J. Appl. Polym. Sci. 2004, 92, 1208-1218.

(231) Adhikari, R.; Michler, G. H.; Henning, S.; Godehardt, R.; Huy, T. A.; Goerlitz, S.; Knoll, K. J. Appl. Polym. Sci. 2004, 92, 1219-1230.

(232) Adhikari, R.; Lach, R.; Michler, G. H.; Weidisch, R.; Knoll, K. Macromol. Mater. Eng. 2003, 288, 432-439.

(233) Adhikari, R.; Michler, G. H. Prog. Polym. Sci. 2004, 29, 949-986.

(234) Adhikari, R.; Michler, G. H.; Knoll, K. Polymer 2004, 45, 241-246.

(235) Shi, W.; Lynd, N. A.; Montarnal, D.; Luo, Y.; Fredrickson, G. H.; Kramer, E. J.; Ntaras, C.; Avgeropoulos, A.; Hexemer, A. 
Macromolecules 2014, 47, 2037-2043.

(236) Shi, W.; Hamilton, A. L.; Delaney, K. T.; Fredrickson, G. H.; Kramer, E. J.; Ntaras, C.; Avgeropoulos, A.; Lynd, N. A.; Demassieux, Q.; Creton, C. Macromolecules 2015, 48, 5378-5384.

(237) Shi, W.; Fredrickson, G. H.; Kramer, E. J.; Ntaras, C.; Avgeropoulos, A.; Demassieux, Q.; Creton, C. ACS Nano 2016, 10, 2054-2062.

(238) Moad, G.; Rizzardo, E.; Thang, S. H. Polym. Int. 2011, 60, 9-25.

(239) Barner-Kowollik, C.; Davis, T. P.; Heuts, J. P. A.; Stenzel, M. H.; Vana, P.; Whittaker, M. J. Polym. Sci. Part A Polym. Chem. 2003, 41, 365375.

(240) Michler, G. H.; Adhikari, R.; Lebek, W.; Goerlitz, S.; Weidisch, R.; Knoll, K. J. Appl. Polym. Sci. 2002, 85, 683-700.

(241) Adhikari, R.; Buschnakowski, M.; Lebek, W.; Godehardt, R.; Michler, G. H.; Calleja, F. J. B.; Knoll, K. Polym. Adv. Technol. 2005, $16,175-182$.

(242) Mittal, V. Materials 2009, 2, 992-1057.

(243) Paul, D. R.; Robeson, L. M. Polymer 2008, 49, 3187-3204.

(244) Zhao, H.; Shipp, D. A. Chem. Mater. 2003, 15, 2693-2695.

(245) Zhao, H.; Argoti, S. D.; Farrell, B. P.; Shipp, D. A. J. Polym. Sci. Part A Polym. Chem. 2004, 42, 916-924.

(246) Zhao, H.; Farrell, B. P.; Shipp, D. A. Polymer 2004, 45, 4473-4481.

(247) Salem, N.; Shipp, D. A. Polymer 2005, 46, 8573-8581.

(248) Morgan, A. B.; Gilman, J. W. J. Appl. Polym. Sci. 2003, 87, 13291338.

(249) Robello, D. R.; Yamaguchi, N.; Blanton, T.; Barnes, C. J. Am. Chem. Soc. 2004, 126, 8118-8119.

(250) Hayashi, M.; Noro, A.; Matsushita, Y. Macromol. Rapid Commun. 2016, 37, 678-684.

(251) Moad, G.; Chiefari, J.; Chong, Y. K. (Bill); Krstina, J.; Mayadunne, R. T.; Postma, A.; Rizzardo, E.; Thang, S. H. Polym. Int. 2000, 49, 9931001.

(252) Rzayev, J.; Penelle, J. Angew. Chem. 2004, 116, 1723-1726.

(253) Truong, N. P.; Dussert, M. V.; Whittaker, M. R.; Quinn, J. F.; Davis, T. P. Polym. Chem. 2015, 6, 3865-3874.

(254) Blumstein, R.; Murphy, G. J.; Blumstein, A.; Watterson, A. C. J. Polym. Sci. Polym. Lett. Ed. 1973, 11, 21-23. 
(255) Huang, J.; Jia, S.; Siegwart, D. J.; Kowalewski, T.; Matyjaszewski, K. Macromol. Chem. Phys. 2006, 207, 801-811. 


\section{Acknowledgements}

First of all, I would like to thank my supervisor Prof. Dr. Philipp Vana for his remarkable support during the past three years. I am grateful for the exciting topic, constant motivation and inspiring discussions. Also, I am deeply indebted to him for giving me the opportunity to study abroad during the time of my thesis.

I also want to thank my co-supervisor Prof. Dr. Konrad Samwer for his kind support, interesting discussions and his deep interest in my topic, which was very encouraging.

I would like to thank all persons who made my stay in Potsdam at Clarkson University become an incredible personal and scientific experience. First and foremost, I want to express my sincere gratitude to Prof. Dr. Devon A. Shipp for hosting me and providing me with extraordinary support during my research stay. I also want to thank Prof. Dr. Mario Wriedt for giving me the possibility to use the facilities of his laboratory. Therefore, I want to thank Hubert Konrad for helping me with the use of the equipment and many interesting and entertaining discussions. I sincerely want to thank Christopher Plunkett for his advice on the TEM sample preparation and measuring the TEM sections.

I want to thank Volker Meyer and all co-workers of the workshop for providing me with countless teflon moulds for the preparation of tensile samples. I am very grateful for the contstruction of the temperature chamber, which contributed tremendously to the success of my work. In this respect I would also like to thank Dr. Hans-Peter Vögele for his technical and theoretical advice, and for many interesting and challenging discussions. I kindly thank Heike Rohmann and Sandra Lotze for a lot practical support.

I would like to thank Bastian Ebeling and Christian Roßner for numerous discussions throughout my doctoral thesis. Their outstanding scientific way of thinking has been both inspiration and motivation.

I want to express my sincere gratitude to all of my bachelor students, who contributed significantly to the success of my work: Lars 
Lewerdomski (chapter 3), Lennart Reuter (section 4.2) and Darius Rohleder (section 4.3). It was a pleasure supervising such ambitious and highly talented students.

I really enjoyed my time in the Vana/Buback group and I want to thank all of my current and former colleagues for the great working atmosphere. In particular, I want to thank Judith Steinhoff, Alex Groschopp, Peng and Jannik Wagner for the pleasant and diverting atmosphere in our office.

I also want to thank Julia Möhrke, Judith Steinhoff, Lennart Reuter, Darius Rohleder, Christian Roßner and Jannik Wagner for carefully proofreading this work.

During the demanding time of my doctoral thesis, it was always a pleasure having the possibility to make music with a lot of fantastic people. I want to thank Isabella Bonas, Jannik Wagner, Jan Fehrs, Franz Kollipost, Benjamin Schomburg and Sebastian Weske for sharing many exciting gigs and songwriting sessions.

Finally, I want to express my deepest gratitude to my family for the unconditional support during the times of my studies and my doctoral thesis. 


\section{About the Author}

Michael Hendrich, * March 31, 1990, Magdeburg, Germany.

\section{Curriculum Vitae}

2008 Abitur (final secondary-school examinations), Werner-vonSiemens-Gymnasium Magdeburg, final grade: "1,5".

2008-2011 Chemistry, Bachelor of Science, bachelor thesis: "Chiralitätserkennung bei Carvon: Ein einfaches Modellsystem für den Geruchssinn" in the group of Prof. Dr. Martin Suhm, final grade: " 1,7 ".

2011-2013 Chemistry, Master of Science, master thesis: "Synthese und Eigenschaften von biomimetischen Multiblock-Copolymeren" in the group of Prof. Dr. Philipp Vana, final grade: "1,3".

2013-2016 Promotion (Ph.D. student), Georg-August-Universität Göttingen, supervisor: Prof. Dr. Philipp Vana.

\section{Publications}

- "Flipping the Pressure- and Temperature-Dependent CloudPoint Behaviour in the Cononsolvency System of Poly- $(\mathrm{N}$ isopropylacrylamide) in Water and Ethanol"

B. Ebeling, S. Eggers, M. Hendrich, A. Nitschke, P. Vana, Macromolecules 2014, 47(4), 1462-1469.

- "Biomimetic Triblock and Multiblock Copolymers Containing L-Phenylalanine Moieties Showing Healing and Enhanced Mechanical Properties"

M. Hendrich, L. Lewerdomski, P. Vana, Journal of Polymer Science Part A: Polymer Chemistry 2015, 53(24), 2809-2819. 